\title{
Evaluation of the Effectiveness of Recovery Methods of Trace Evidence for Pollen Particles
}

\author{
Christie Cyktor
}

Follow this and additional works at: https://researchrepository.wvu.edu/etd

\section{Recommended Citation}

Cyktor, Christie, "Evaluation of the Effectiveness of Recovery Methods of Trace Evidence for Pollen Particles" (2015). Graduate Theses, Dissertations, and Problem Reports. 5426.

https://researchrepository.wvu.edu/etd/5426

This Thesis is protected by copyright and/or related rights. It has been brought to you by the The Research Repository @ WVU with permission from the rights-holder(s). You are free to use this Thesis in any way that is permitted by the copyright and related rights legislation that applies to your use. For other uses you must obtain permission from the rights-holder(s) directly, unless additional rights are indicated by a Creative Commons license in the record and/ or on the work itself. This Thesis has been accepted for inclusion in WVU Graduate Theses, Dissertations, and Problem Reports collection by an authorized administrator of The Research Repository @ WVU. For more information, please contact researchrepository@mail.wvu.edu. 


\title{
Evaluation of the Effectiveness of Recovery Methods of Trace Evidence for Pollen Particles
}

\author{
Christie Cyktor, B.S. \\ Thesis submitted \\ to the Eberly College of Arts and Sciences \\ at West Virginia University \\ in partial fulfillment of the requirements for the degree of \\ Master of Science in \\ Forensic \& Investigative Science \\ Dr. Keith Morris, Ph.D., Chair \\ Dr. Patrick Buzzini, Ph.D., Thesis Mentor \\ Dr. Jack Hietpas, Ph.D. \\ Dr. Gerald Lang, Ph.D. \\ Department of Forensic and Investigative Sciences \\ Morgantown, West Virginia \\ 2015
}

Keywords: trace evidence, pollen, dust, collection methods, recovery techniques

Copyright 2015 Christie Cyktor, B.S. 


\begin{abstract}
Evaluation of the Effectiveness of Recovery Methods of Trace Evidence for Pollen Particles

Christie Cyktor, B.S.
\end{abstract}

Within the forensic science community, pollen as a form of trace evidence is extremely underutilized. In many instances, trace evidence examiners and crime scene investigators are unfamiliar with how best to recover pollen from a piece of evidence. Methods such as vacuum sweeping, tape lifting, and sonication have been implemented for the recovery of the test dust from materials such as clothing, shoes, or improvised explosive devices. While these methods are known to be beneficial with some trace materials, their effectiveness with pollen has yet to be determined. The goal of this research project was to implement and compare multiple sampling techniques for pollen incorporated into a test dust on various substrates in an effort to establish which technique was most effective at recovering the greatest amount of the pollen/dust mixture. In this research pine pollen was incorporated in to a "test dust" that was applied to five different forensically relevant surfaces: two different brands of a cotton knit shirt, $100 \%$ nylon stockings, metal cans, and shoes - all of which may be encountered at crime scenes. Through this work, it was determined that the tape lift method most effectively removed the test dust off of all of the surfaces examined. The effectiveness was based on the speed of the recovery technique as well as what method removed the greatest amount of pollen. 


\section{Acknowledgements}

I would like to thank all of my committee members, especially Dr. Patrick Buzzini, for their guidance during my thesis project, as well as the West Virginia Space Grant Consortium for providing me with a grant that enabled me to present my work at the 2015 Impression, Pattern, and Trace Evidence Symposium. 


\section{Contents}

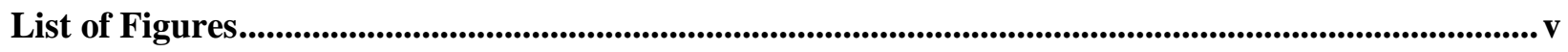

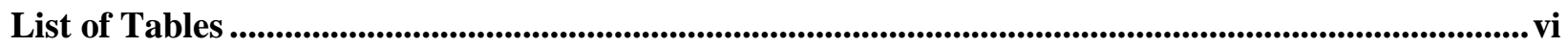

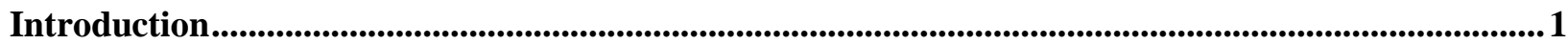

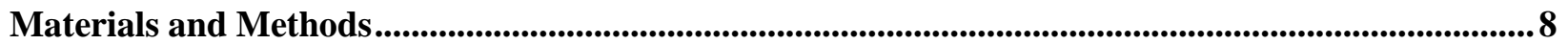

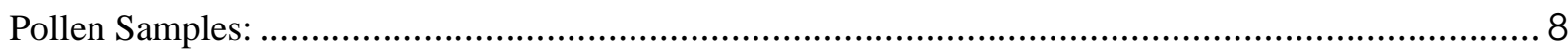

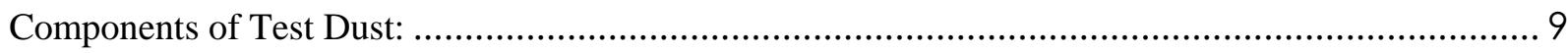

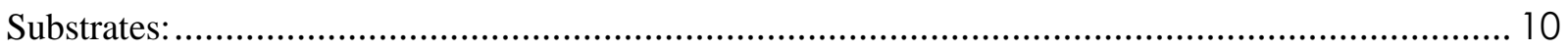

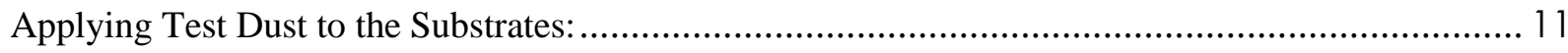

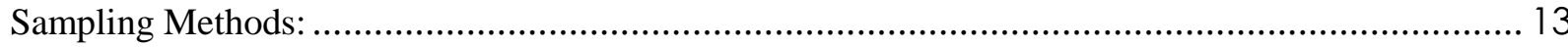

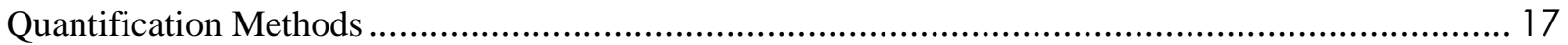

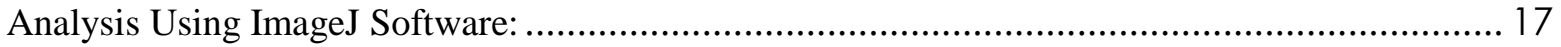

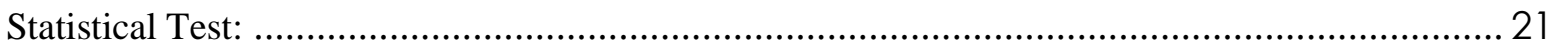

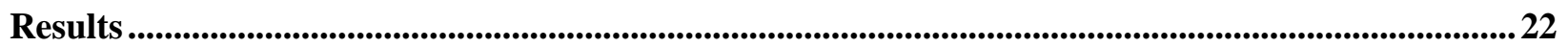

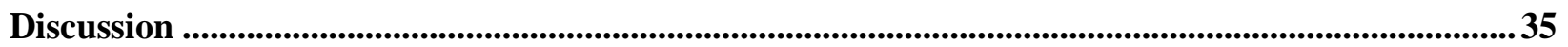

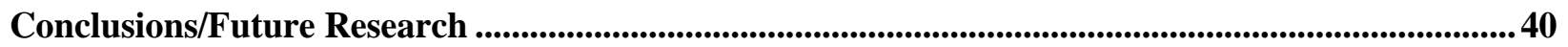

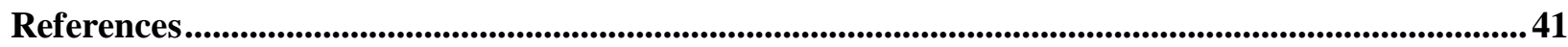

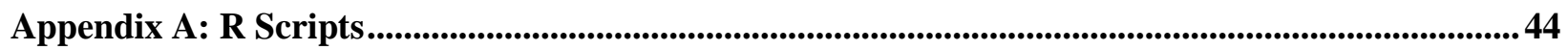

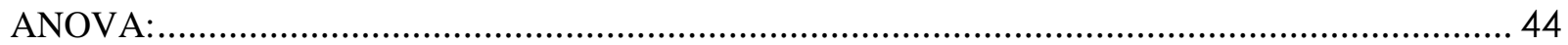

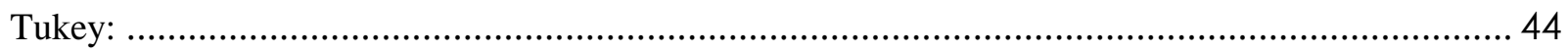

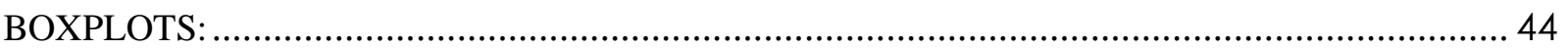

Appendix B: Tables of Pollen Counts ....................................................................................................................45

Appendix C: Image Acquisition................................................................................................................................186 


\section{List of Figures}

Figure 1: Image of angiosperms (left) and gymnosperms (right) ${ }^{7}$......................................... 5

Figure 2: Pine pollen stained using the malachite green solution staining method (10x obj.) ................ 8

Figure 3: Test dust under fluorescence filters A (top left), D (middle), E4 (top right), I3 (bottom left), and

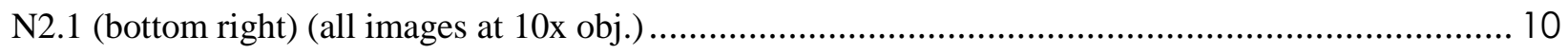

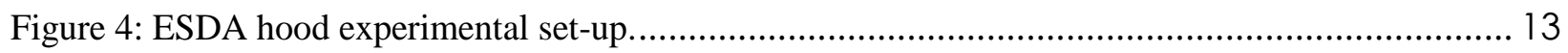

Figure 5: Process of performing the tape lift method on one tennis shoe. .................................... 14

Figure 6: Process of performing the vacuum filtration method on one tennis shoe. ........................... 15

Figure 7: Process of performing the sonication method. .................................................... 16

Figure 8: Example of the image transformation. Photomicrographs of the stained pollen recovered from

Fruit of the Loom t shirt via tape lift 8.36 (left) and binary image of tape lift (8.36) (right). (10x obj.) ... 18

Figure 9: Photomicrographs of the binary image of Fruit of the Loom t shirt via tape lift 8.36 (left) and eroded image of tape lift (8.36) (right). (10x obj.) ................................................................... 19

Figure 10: "Cell Counter" code and examples of validation image 1 (left) and validation image 2 (right)

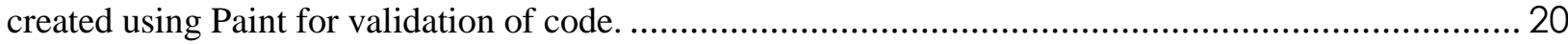

Figure 11: Boxplot showing the means of the amount of pollen found on all surfaces using the three

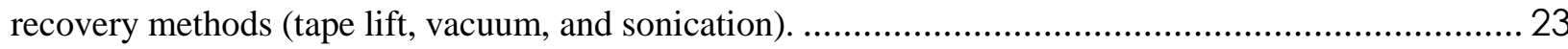

Figure 12: Boxplot showing the means of the amount of pollen found on each surface by each recovery

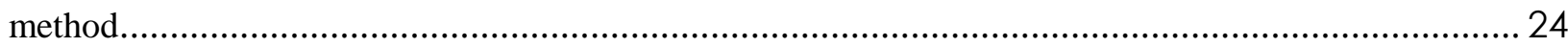

Figure 13: Boxplot showing the mean amount of pollen collected by each method used on every surface

(tape_lift and vacuum) as a whole ..................................................................................... 25

Figure 14: Histogram of amounts of pollen collected from fruit of the loom $t$ shirts........................... 26

Figure 15: Density curve illustrating pollen collected from fruit of the loom t shirts .......................... 26

Figure 16: Histogram of amounts of pollen collected from hanes $t$ shirts ...................................... 27

Figure 17: Density curve illustrating pollen collected from hanes t shirts ....................................... 27

Figure 18: Histogram of amounts of pollen collected from nylons .............................................. 28

Figure 19: Density curve illustrating pollen collected from nylons ............................................. 28

Figure 20: Histogram of amounts of pollen collected from tennis shoes........................................ 29

Figure 21: Density curve illustrating pollen collected from tennis shoes ........................................ 29

Figure 22: Histogram of amounts of pollen collected from cans ................................................ 30

Figure 23: Density curve illustrating pollen collected from cans ............................................... 30

Figure 24: Graphical representation of tukey results obtained for comparison of means between all

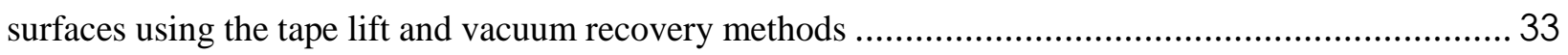

Figure 25: Graphical representation of tukey results for the means of recovery methods ..................... 34

Figure 26: Graphical representation of tukey results for means of surfaces .................................... 35 Figure 27: Density curve for the amounts of pollen collected using the tape lift method for both brands of

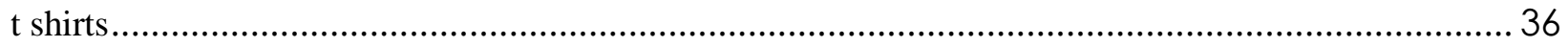




\section{List of Tables}

Table 1: Filter cube specs for Leica Microsystems. ................................................................ 9

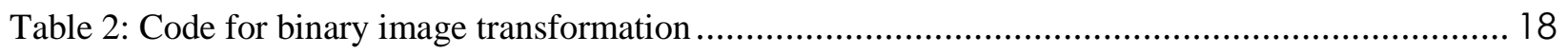

Table 3: Summary anova results for amounts of pollen collected using all tape lift and vacuum methods

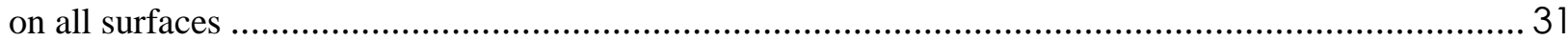

Table 4: Tukey results for all recovery methods and surfaces................................................... 32 


\section{Introduction}

Trace evidence examination is a critical aspect of forensic science, police investigations, and police intelligence. Often times an assailant may carry away with them, or pick up, microscopic particles that could assist in providing a linkage between people and locations. A few of the main types of trace evidence that are often encountered are hair, fibers, paint, and glass; however, a particle that may be considered underutilized is a grain of pollen. These inconspicuous particles can persist on a multitude of surfaces for several weeks or months, however it is an aspect of trace evidence examination that does not get utilized as frequently as other forms of evidence ${ }^{1}$. Many analysts and crime scene investigators are unfamiliar with how best to collect pollen evidence associated with forensic cases. In a scenario where DNA, fibers, or glass are present, which would be quite useful for identification, pollen could be used as a means of providing a vital investigative lead about a certain geographical location for forensic intelligence. Pollen is a unique substance that varies significantly across the world, but also within local geographical areas ${ }^{2}$. Depending on the amount of foot traffic or air circulation in a certain area, pollen profiles may vary significantly even if the areas in question are in close proximity to one another - thus providing a unique signature of a specific area.

The main focus of this research project was to implement and compare multiple sampling techniques for pollen incorporated into a test dust on various substrates in an effort to establish which technique is most effective at recovering the greatest amount of the pollen/dust mixture. While this is the overall goal of the study, the impact of the findings will be beneficial to criminalists and crime scene investigators on a much wider scale. Evidence recovery is a crucial aspect in the forensic science community and can pose a problem if the criminalist is unfamiliar 
with different recovery methods available to them. Proper sampling is vital to complete an accurate data interpretation.

Common recovery methods included taking tape lifts using a soluble tape, using a vacuum filtration system, and lastly sonication. These methods are employed to sample various forms of trace evidence and will be useful to gauge the strengths and weaknesses of each sampling procedure as a function of evidence type. In forensic applications it is important to recover as much evidence as possible. The most appropriate sampling method may vary depending both on the substrate and the examiner's goals and personal methodological preference.

Collecting and analyzing trace evidence in a forensic scenario can be of tremendous benefit when investigating a wide array of possible crimes. The most common to consider would be a hit and run accident, theft, or perhaps an act of vandalism. However, crimes that involve gunfire are also seen by a trace evidence examiner. For these various crimes a multitude of trace substances may be encountered such as fibers, hairs, glass fragments, paint fragments, gunshot residue particles, and soil minerals or pollen. A trace analyst may need to specialize in all of these types of evidence, therefore it is essential to have knowledge of the strengths and weaknesses of various collection methods that may be appropriate depending on the substrate encountered when investigating a crime.

One method for collecting trace evidence is to use a soluble tape. The tape is repeatedly applied to a particular surface (furniture, a car seat, a garment, etc.) and then placed on a transparent sheet for future analysis. Once the findings are sent to the laboratory for analysis a chemical solution, such as xylene, is applied to dissolve the adhesive side of the tape so that the questioned particles can be isolated for microscopic analyses. This form of recovery is 
beneficial because it allows for rapid scene processing and effectively gathers the questioned samples, but is nonspecific and prone to detecting significant "noise", or background particles that may not be relevant in the context of the case. If an examiner wishes to collect black fibers from a car seat but the tape lift reveals groups of fibers that are many different colors, then the tape lift may end up producing additional work for the analyst due to the fact that he/she would then have to closely examine the tape to ensure the proper fiber samples were collected. Additionally, it could be possible that the adhesive backing of the tape may not dissolve completely in the xylene, making it difficult to remove the trace evidence samples without physical alteration. Despite the drawbacks of using adhesive tape to gather evidence the rapid collection time and ease of use make this collection method extremely beneficial to the analyst ${ }^{3,}$ 4.

In the collection process of physical evidence it is occasionally necessary to inspect and sample a large surface or to extract embedded particles that may not have been recovered using a tape lift. In these instances a vacuum filter may be used to collect soil, hairs, or fibers. In order to collect the samples a standard vacuum hose can be equipped with an air filter cassette with a clear methacrylate plastic that allows fine particles to pass through. On the top of the cassette is a piece of filter paper used to prevent large particles from passing through, on the other side is the nozzle that is passes over the surface in question. The filter paper is removed from the apparatus after the vacuum has been run and the contents are examined under a microscope. This method of collection is both rapid (large surfaces such as clothing or vehicles can be sampled in a short period of time), and convenient (the filter cassettes can be pre-purchased or easily assembled). 
However, a weakness of the vacuum method is that there is the potential for collecting irrelevant evidence because the entire surface will be processed and fragments from the distant past may be gathered. If a visual inspection was performed instead and the evidence was removed using forceps, or a tungsten needle, the process would be much more selective and there would be virtually no background interference; however, hand-selection is significantly more time consuming and not appropriate when the particles of interest are too small to allow for reasonable collection with forceps ${ }^{5}$.

The final collection method that was examined in this study was the use of sonication, which is known to be beneficial in cases involving clothing with embedded oil. To execute this method, a clipping from the material of interest (e.g. a sock) can be taken and suspended in an Eppendorf tube with distilled de-ionized water. The tube can then be sonicated to promote disaggregation. The tube can then be centrifuged for an additional cycle and the fabric and supernatant removed/decanted revealing a sediment pellet for examination. It should be noted that this is a common practice in the oil industry as well as paleoecology, but the literature does not make note of it for use with soil or dust samples. Its use in the forensic setting would be beneficial because it should successfully remove evidence that may be imbedded deep within the fabrics of certain clothing ${ }^{6}$.

A wide range of evidence can be collected using a number of the methods listed, this study will focus on pollen, and on a wider scale, dust. Dust can accumulate on various surfaces in an indoor setting while pollen has a prominent location outside. The use of dust/pollen can help in connecting a person to a certain location even if that person claims to have been at a different location at the time of a crime. Pollen comes in the form of small particles from plants, flowers, or trees and can vary greatly depending on the geographical location. 
Palynology is the study of pollen; its morphology, composition of peat and sediment, and how it can disperse from location to location. The two categories of pollen are angiosperms and gymnosperms. Angiosperms are referred to as flowering plants that have seeds that are enclosed within a fruit. Gymnosperms have no flowers or fruits, but rather they have naked seeds on the surface of their leaves or cones (see Figure 1). The key characteristic that distinguishes angiosperms from gymnosperms is the presence of flowers or fruits. Because of the large number of varieties of the fruit and/or flower-bearing plants, angiosperms have different colors and shapes of leaves, flowers and fruits. Gymnosperms have spiky, needle-like leaves, and are also simpler physically because they do not bear flowers or fruit. Although there are different species, gymnosperms are usually only tall evergreens with brown cones.
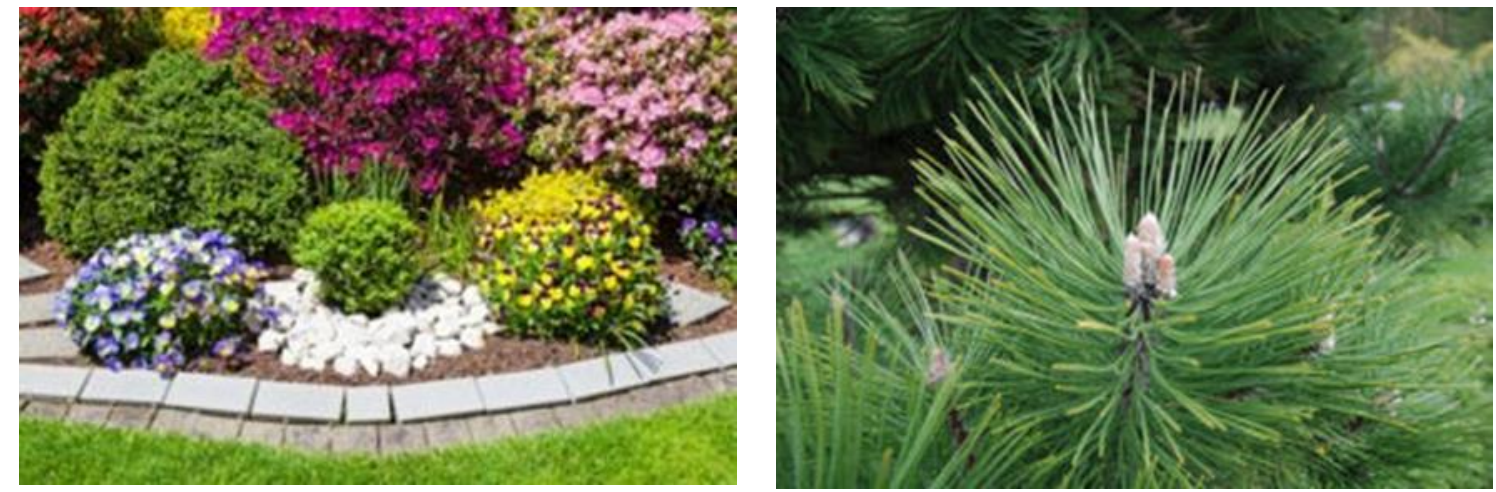

FIGURE 1: IMAGE OF ANGIOSPERMS (LEFT) AND GYMNOSPERMS (RIGHT) ${ }^{7}$

Different plant types will have differences in their pollen grain structure, and as a result may lose pollen in different ways. In this work pine pollen was used which derives from the gymnosperm plant type, however the type of plant may have an effect on the success of a particular recovery method. The different structures of pollen make the grains prime candidates for the type of evidence that can be carried to different locations via the wind or water. Due to this fact, pollen 
and dust serve as important pieces of physical evidence that a perpetrator may not even know they carried away from a crime scene ${ }^{8}$.

One type of scenario in which pollen could be used as a means of investigating a crime would be with the use of clothing if a particular location of a crime is in question. This is particularly seen if a dead body is recovered from an area that is not believed to be the area in which the death occurred or in a situation of rape. In the case of rape it is possible that the location of the assault could be called in to question. In one scenario, a woman claimed that she was raped in a small alleyway between two buildings, however the assailant claimed that he never left the area of his car that was parked approximately seven meters away. To conduct the analysis the pants of the assailant were analyzed using standard potassium hydroxide deflocculation. The driveway was located next to a busy street whereas the alleyway was positioned between two large buildings that did not allow for a great deal of air flow. The pollen types of the alleyway had a particular profile, namely Coprosma that was not found in the driveway. The stain on the assailant's pants matched the Coprosma found in the alleyway and thus linked him to the rape, illustrating how areas of vegetation that are relatively close can have varying pollen profiles ${ }^{9}$.

In an effort to gauge the effectiveness of a particular collection method, the presence of particles will be examined. The theory of particle counting has been shown by researchers in the indoor air quality community, particularly with fungal growth indoors. In homes that have experienced water damage or ventilation it is common to see hazardous indoor fungal growth. While information about the types of culturable bacteria that can be found in a home with fungal issues is readily available, there is currently no record of an acceptable "standard" in a clean home. To account for this, fifty homes that were known to have no pre-existing issues with 
indoor fungus were examined over the course of one year. Samples were taken inside the home during the winter and summer months to consider the temperature effects when either the heat or the air conditioning was running. The presence of air or dust-borne mycoflora was measured to determine whether or not fungal growth could become an issue in these homes. Once samples of the dust within the home, the air vents, and an area directly outside had been collected the samples were analyzed for the presence/abundance of fungi. The cultures taken from the home were plated and bacterial colonies were analyzed to determine the various taxa present. The mean amount of bacteria was determined for each sample taken at the different times of collection and an ANOVA (analysis of variance) was run. The study showed that air-borne dust inside greatly reflected fungal specimens taken from outdoors, both in prevalence ranking and in abundance ranking. This was significant because it showed that the bacteria outside was able to grow indoors, indicating that ventilation and water damage was present in homes that were previously believed to be clean. While some results gathered were skewed and/or higher than what was previously expected, this study was useful in determining that through visual inspection the presence and abundance of certain bacterial colonies could be determined ${ }^{10}$.

The main goals and objectives of this research were to determine which recovery methods were most useful for different surfaces, provide forensic examiners insight as to which recovery methods were most efficient at collecting pollen for analysis, successfully depict "reallife" scenarios through the use of cotton t-shirts, tennis shoes, $100 \%$ nylon stockings, and paint cans, as well as to determine a quantitative ranking of effectiveness among recovery methods. 


\section{Materials and Methods}

Pollen Samples:

Pollen was the main component that was studied in this research in an effort to focus on one type of evidence that could be commonly encountered, however the approach could be used on many different forms of evidence. Pine pollen was purchased from an online supplier and was the chosen type of pollen for this study due to its prevalence throughout North America ${ }^{11}$. Only a small amount of pollen was necessary for deposition on the selected substrates, however replicate samples were needed. The pollen grains were stained prior to inclusion with the test dust and the substrates using a stain known as malachite green. This stock stain solution was prepared by combining $90 \mathrm{~mL}$ of ethanol, $10 \mathrm{~mL}$ of deionized water, and 1 gram of malachite green salts; $1 \mathrm{~mL}$ of the stock solution was combined with the pine pollen and mixed thoroughly. This stained the grains a green hue and allowed for relatively facile identification of that particular grain ${ }^{12}$. An image of how the pollen absorbed the stain is shown in Figure 2.

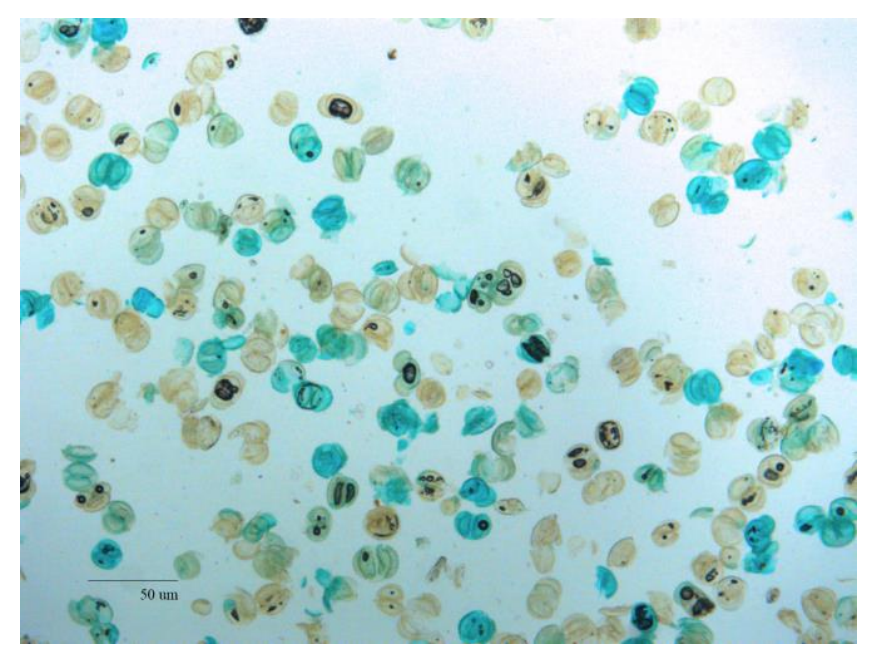

FIGURE 2: PINE POLLEN STAINED USING THE MALACHITE GREEN SOLUTION STAINING METHOD (10X OBJ.) 


\section{Components of Test Dust:}

To allow for fluorescence of the pollen grains under UV radiation a test dust was

prepared. Calcite (white) contains calcium carbonate and should fluoresce differently than the pollen grains. The calcite was used to make the test dust in order to give more weight to the dust as well as to offer a contrast to the pollen grains under fluorescence. The calcite was originally clumped together in miniscule rocks. The "dust" was prepared by crushing the rocks using a mortar and pestle. The calcite powder was then sieved into a very fine powder, similar to the consistency of pine pollen powder. This calcite dust was dispersed evenly over the substrate using an ESDA aerosol development hood. The dust recovered from the different substrates by various methods was placed under the fluorescence microscope and analyzed using the UV filters N2.1, I3, D, A, and E4 (see table below for specs from Leica Microsystems). The "A" caused the pollen grains to fluoresce blue, the "I3" and "D" filters both caused the pollen grains to fluoresce green, the "E4" filter caused a green/blue fluorescence, and the "N2.1" filter caused the pollen grains to fluoresce red. The images of the test dust in Figure 3 illustrate this fluorescence. The fluorescence of the calcite was intended to aid in the differentiation between a pollen grain and a calcite particle, however after experimentation it was observed that the two could not be differentiated from one another based on fluorescence. As a result, the fluorescence images were not used during subsequent image analysis.

Table 1: Filter cube specs for Leica Microsystems.

\begin{tabular}{|c|c|c|c|c|}
\hline Filter Cube & Excitation Range & $\begin{array}{c}\text { Excitation filter } \\
(\mathbf{n m}\end{array}$ & $\begin{array}{c}\text { Dichromatic } \\
\text { mirror }(\mathbf{n m})\end{array}$ & $\begin{array}{c}\text { Suppression filter } \\
(\mathbf{n m})\end{array}$ \\
\hline A & UV & BP 340-380 & 400 & LP 425 \\
\hline D & UV/violet & BP 355-425 & 455 & LP 470 \\
\hline E4 & Violet/blue & BP 436/7 & 455 & LP 470 \\
\hline I3 & Blue & BP 450-490 & 510 & LP 515 \\
\hline
\end{tabular}




\begin{tabular}{|l|l|l|l|l|}
\hline N2.1 & green & BP 515-560 & 580 & LP 590 \\
\hline
\end{tabular}
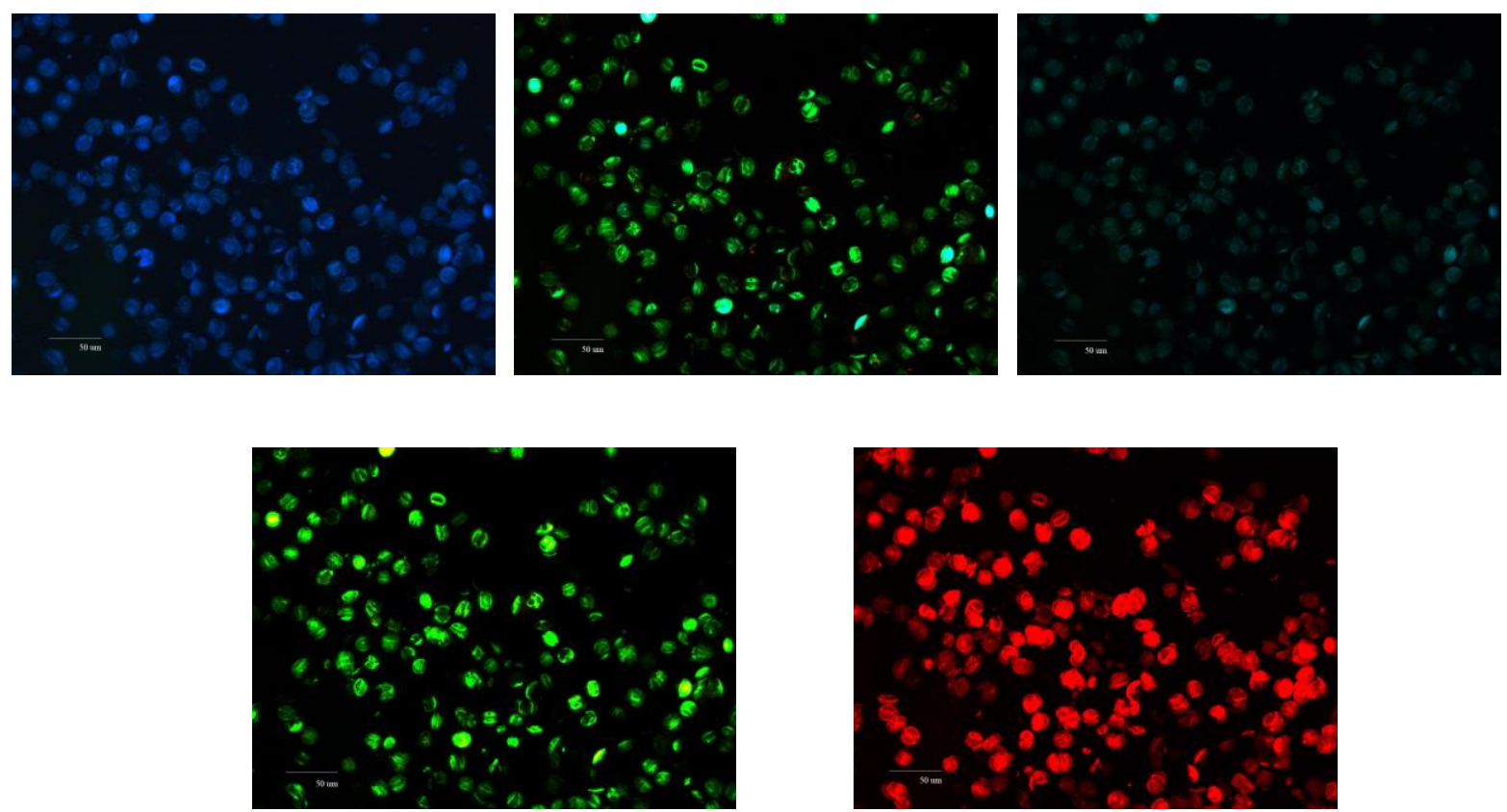

FIGURE 3: TEST DUST UNDER FLUORESCENCE FILTERS A (TOP LEFT), D (MIDDLE), E4 (TOP RIGHT), I3 (BOTTOM LEFT), AND N2.1 (BOTTOM RIGHT) (ALL IMAGES AT 10X OBJ.)

\section{Substrates:}

The research design was meant to mimic applicable forensic settings as closely as possible. For this purpose, the substrates for the dust samples were chosen based on their likelihood of being discovered at a crime scene. This crime could be a rape scenario, a case of counterfeit bank notes, or a case in which other inanimate objects might be present during the commission of a crime. The chosen substrates for examination were $100 \%$ nylon stockings, cotton knit clothing, shoes, and empty metal cans (IED-improvised explosive devise- simulant). Shoes and clothing seemed to be logical choices due to the high probability of finding pollen adhering to them, especially if the crime occurred in an outdoor setting. Furthermore, the stockings and the metal cans offered a scenario that may be less frequently encountered, such as 
if a bomb was discovered in a location other than where it was constructed or a woman's panty hose were recovered from a rape case. All of these substrates would require different sampling techniques. There were thirty-five replicates run on the same substrate, with three analysis methods, and this was done for all substrates. Five different substrates, thirty-five replicates on each substrate, and three different analysis methods yields:

35 replicates $x 5$ substrates $x 3$ sampling methods $=525$ analyses

After preliminary experimentation it was determined that the sonication method was not practical to use with the shoes or the metal cans due to the size of those samples. Therefore, the total analysis number was altered. All three sampling methods were only used on three of the five surfaces:

35 replicates $\times 3$ substrates $\times 3$ sampling methods $=315$ analyses
35 replicates $\times 2$ substrates $\times 2$ sampling methods $=140$ analyses
Total $=455$ analyses

\section{Applying Test Dust to the Substrates:}

A crucial aspect of this study was to ensure that equal amounts of the test dust were dispersed on all of the substrates. This necessitated a confined air space, as air fluctuations throughout the room may create drafts that disperse more test dust on one substrate over another. In order to regulate air flow, the hood of an electrostatic detection apparatus (ESDA) was used. The ESDA hood is an apparatus that has shown to be useful in questioned document analysis through its ability to reveal writing impressions by dispersing a cloud of toner over the document that then settles into the furrows left behind by the writing instrument. The use of the ESDA 
hood in the capacity of pollen dispersal is a novel approach that was designed to mimic the way in which wind would deposit pollen on a particular object. Due to the fact that the chamber was not entirely flush with the bench top a foam pad was placed under it to act as a sealant. The chosen sample was then placed inside the chamber on the foam pad. Figure 4 illustrates the setup of the ESDA hood on the foam pad. The ESDA hood contains a chamber in which the dust/pollen mixture was placed. A hand lever on the top of the chamber was then switched back and forth which caused a felt wheel to turn inside the small chamber. The test dust was gathered by this wheel and passed in to a fan mechanism that circulates once the equipment is turned on. The spinning fan dispersed the dust/pollen mixture throughout the chamber and on to the selected substrate. The test dust sample was prepared by combining approximately equal amounts of calcite and pollen grains; then, roughly 0.2 grams of the dust mixture was placed inside the chamber to be placed on the surfaces. Each cycle of running the ESDA hood took approximately ten minutes per sample to ensure that all of the test dust had completely passed through the chamber and on to the desired surface. The substrate was then removed from under the chamber and the chosen recovery method was immediately performed. 


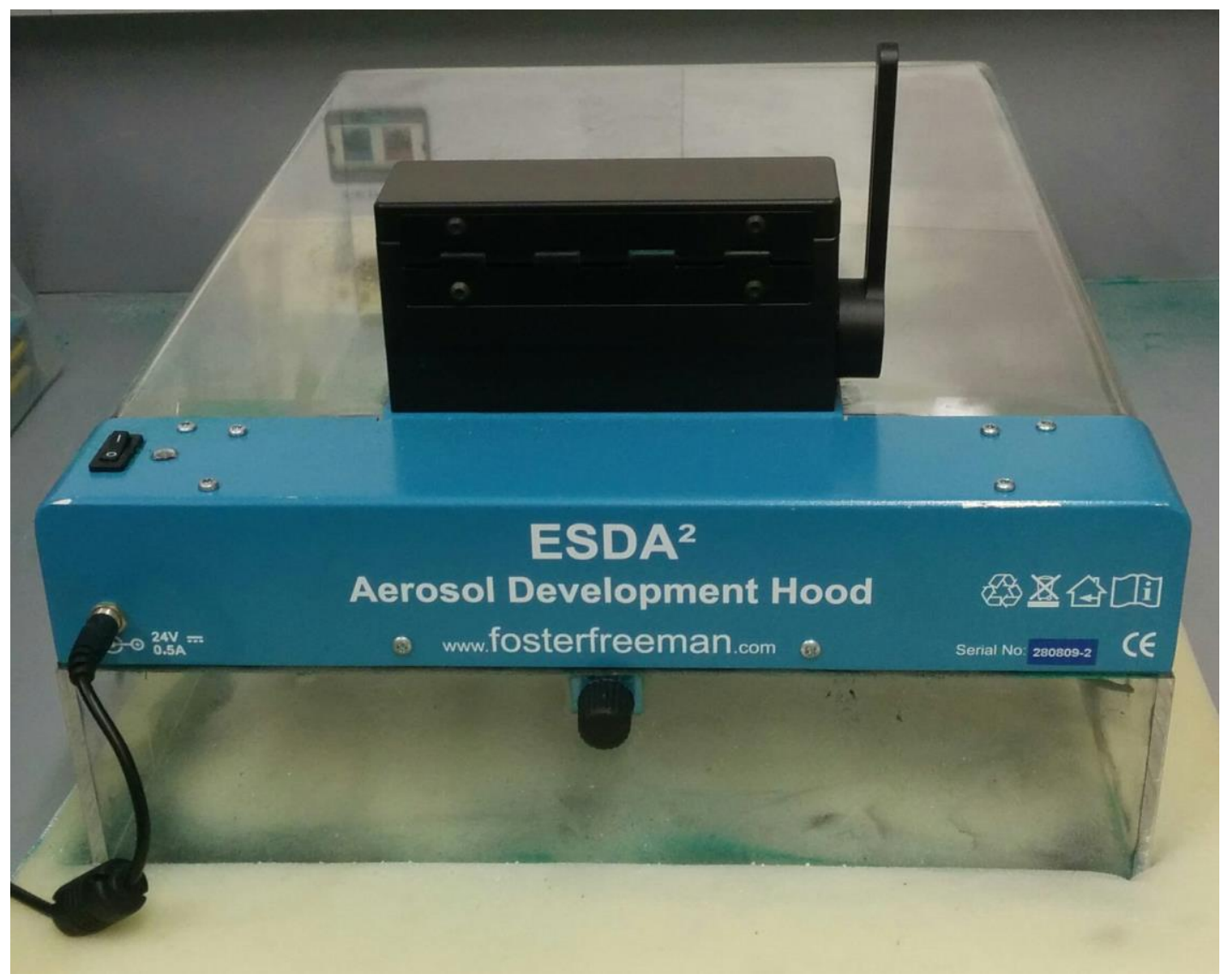

FIGURE 4: ESDA HOOD EXPERIMENTAL SET-UP.

\section{Sampling Methods:}

Three different sampling methods were implemented on the various substrates chosen.

The first of these methods was to use a soluble tape (Sirchie lifting tape) to lift off the test dust; all tape strips used were approximately 3" wide x 6" long. This was done by taking the adhesive side of tape and gently pressing it to the substrate's surface approximately ten times. The tape was then placed on a transparent acetate sheet and was directly viewed under the fluorescence microscope (Leica DM6000, 10x obj.). The image below shows the process of performing the tape lift on one tennis shoe (Women's casual canvas shoe) ${ }^{13,14}$. 


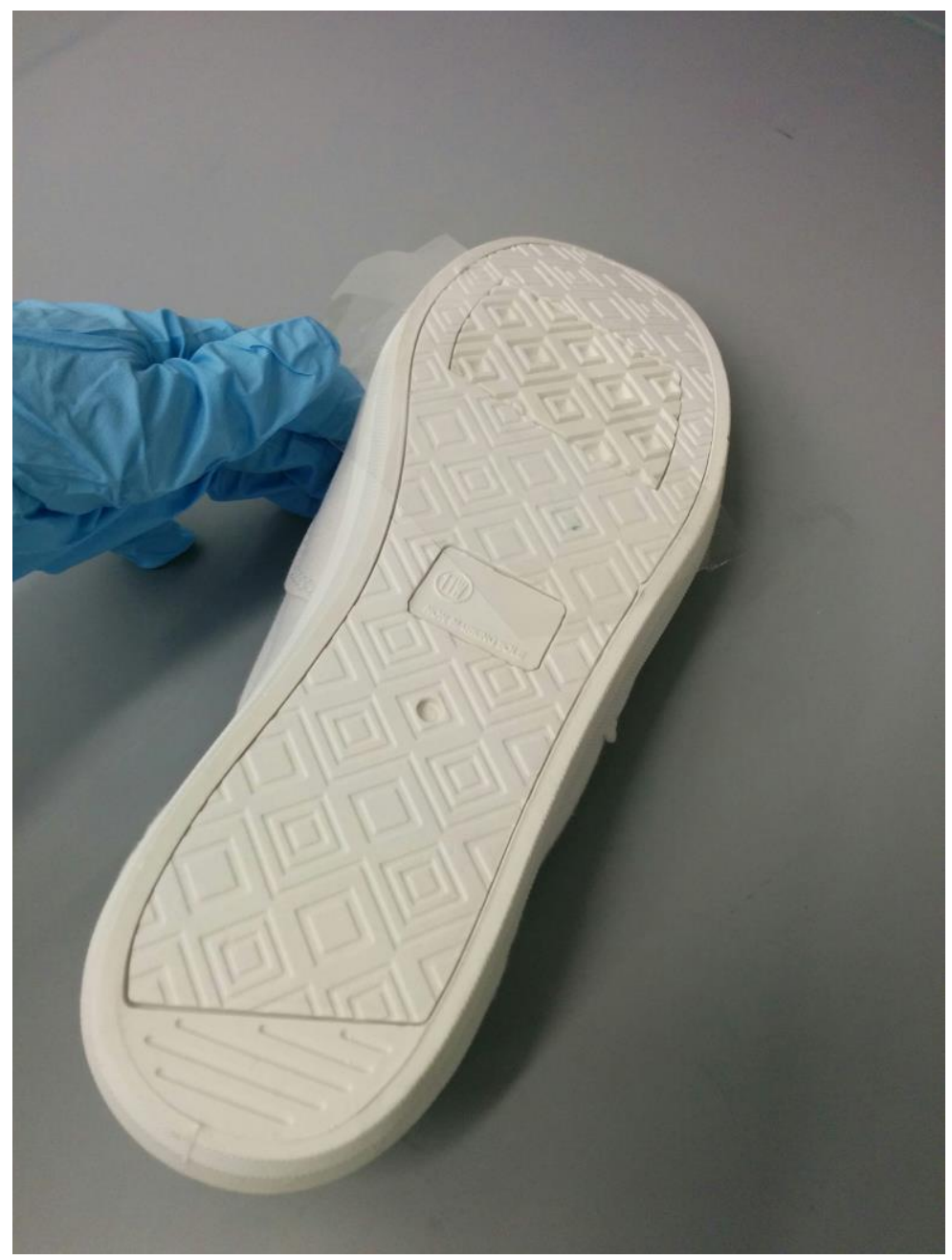

Figure 5: Process OF PERforming THE TAPE LIFT METHOD ON ONE TENNIS SHOE.

The vacuum filtration method was performed by fixing a vacuum cleaner (3M Trace Evidence Collection Vacuum, Model: 4-3005) with a clear methacrylate plastic that allows microscopic debris to pass and a piece of filter paper that is placed in the head of vacuum hose. The surface of the substrate was then vacuumed repeated times to ensure that all particles are collected; after this the head of the vacuum was dismantled and the filter paper was retained for further analysis of the extent to which the particles were collected. 


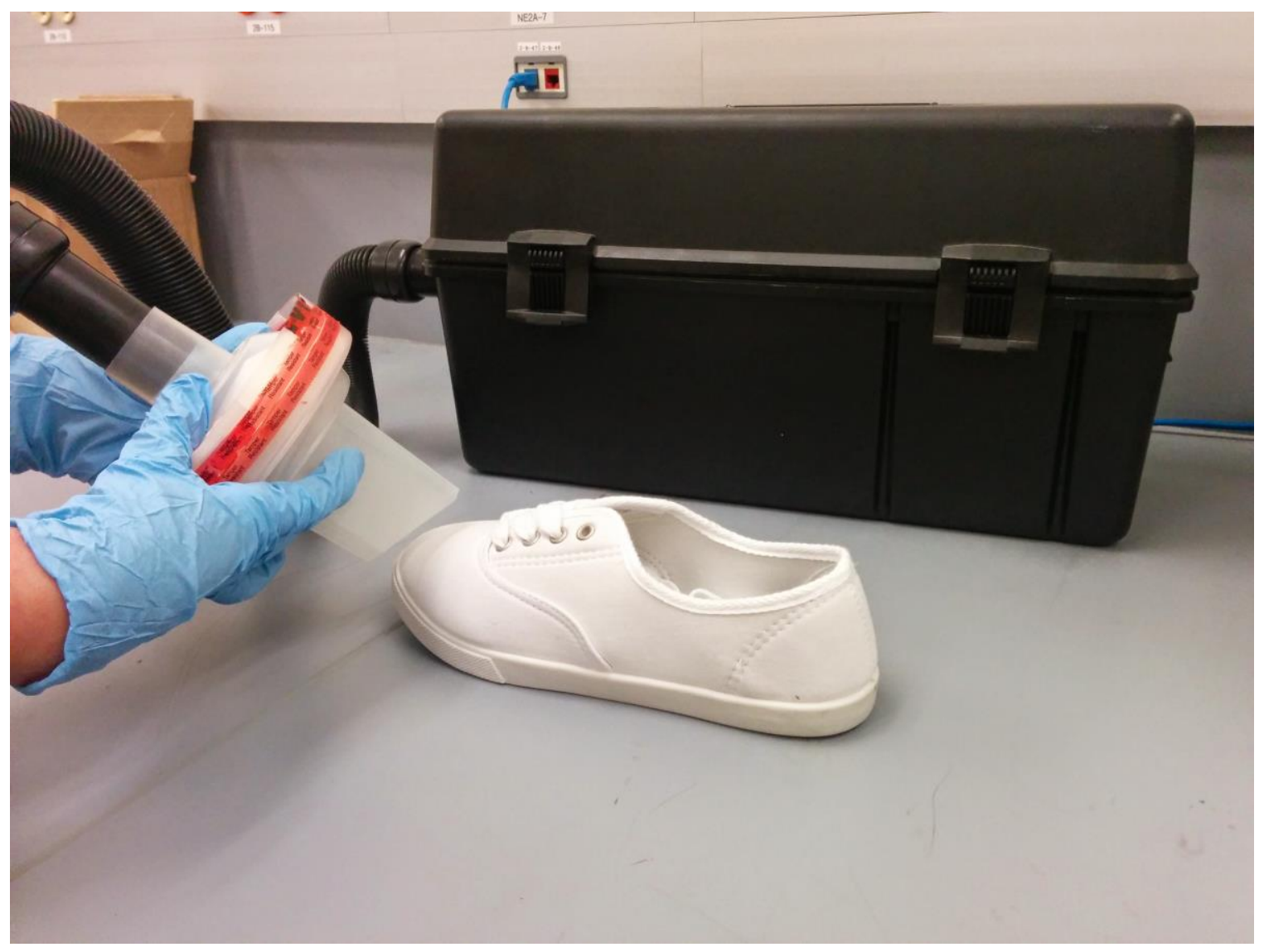

FIGURE 6: PROCESS OF PERFORMING THE VACUUM FILTRATION METHOD ON ONE TENNIS SHOE.

The final analysis method that was used was a sonication method (Branson 200

Ultrasonic Machine, 15 oz. capacity, Mfr. Part \#: B200). As an example, a sock was recovered that contained trace amounts of soil. A sonication method was outlined in which a clipping from the sock was taken and suspended in an Eppendorf tube with a distilled deionized water. The tube was then vibrated for four minutes on an ultrasonic disaggregation machine. The tube was the centrifuged at 4000 RPM for an additional four minutes and the sock and supernatant were decanted off so that the remaining sediment pellet could be examined. This approach is not a standard method for soil and dust recovery, however it could prove to be very useful, particularly for clothing ${ }^{15}$. 


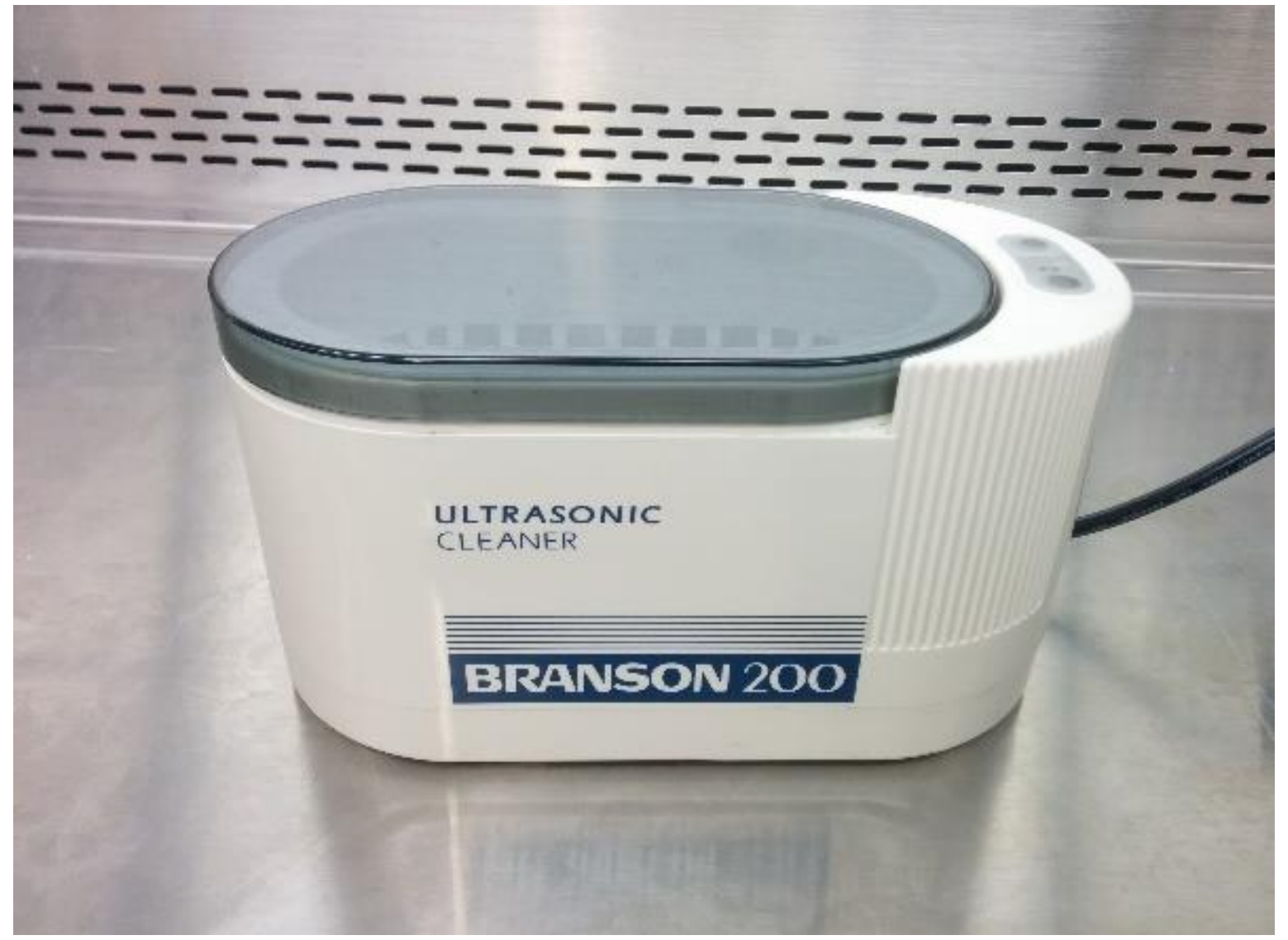

FigURE 7: PROCESS OF PERFORMING THE SONICATION METHOD.

For the sonication method used in this study the chosen substrate was submerged in water and the sonicator was run twice, each run lasting five minutes, to ensure that the maximum amount of test dust was removed from the surface. The substrate was then removed from the sonicator and the remaining water was filtered through standard filter paper to capture the test dust that was removed from the surface and allowed to dry overnight. Due to the fact that the pollen gathered together on the filter paper after drying, it was not able to be viewed directly under the fluorescence microscope because an accurate pollen count could not be made. Instead, the filter paper was weighed before and after sonication and a conversion between weight and pollen grain count was made. The weight of the test dust was known prior to dispersal on the 
various surfaces. In $0.025 \mathrm{~g}$ of test dust there were approximately 1,200 grains of pollen. Using these values, a weight conversion was performed on all filter papers from the sonication samples using the equation below.

(weight of filter paper $w /$ pollen - weight of filter paper w/o pollen $) *\left(\frac{\text { pollen grain count }}{0.025 \text { g pollen }}\right)$

These analysis methods were found to be more beneficial for some substrates rather than others, but the examination of all methods was vital for the success of the method validation.

\section{Quantification Methods}

Analysis Using ImageJ Software:

The main method that was used to illustrate whether or not a particular analysis is viable for pollen recovery was to count the test dust particles using ImageJ software (Image Processing and Analysis in Java, IE 6.0, Microsoft Java 1.1.4.). Each grain of dust will appear as a "point" that can be counted after a particular recovery method is performed. All images captured using the fluorescence microscope were therefore run through various pieces of code using ImageJ that would produce a final count of the grains collected using the different recovery methods.

In order to perform the manipulations necessary to obtain a final count number all of the images taken using the fluorescence microscope had to be converted into binary, or black and white, images. This is a stipulation inherent in the functions of the ImageJ software, therefore the backgrounds of the images were turned to white and any pollen grains became black. This process was tested several times manually by using the various menu operations in ImageJ, however once it proved to be successful a "batch code" was made that would perform the Make Binary operation on all input files. The code and an example of the conversion are shown in Error! Reference source not found.2 and Figure 8 below. 
TABLE 2: CODE FOR BINARY IMAGE TRANSFORMATION
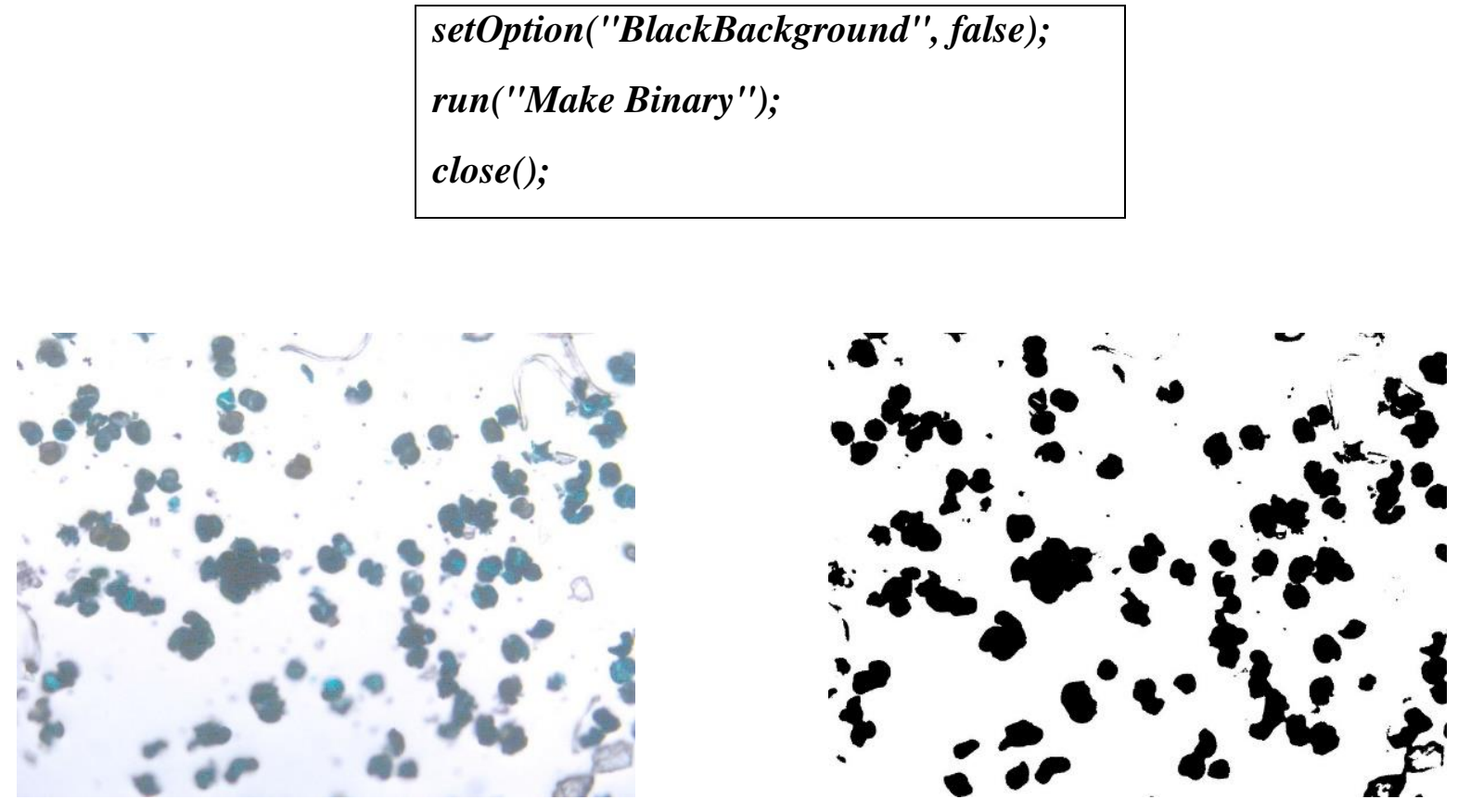

Figure 8: EXAMPle OF THE IMAge TRANSFORMATION. PHOTOMiCROgRAPHS OF THE STAined POLLEN RECOVERED FROM FRUIT OF THE LOOM T SHIRT VIA TAPE LIFT 8.36 (LEFT) AND BINARY IMAGE OF TAPE LIFT (8.36) (RIGHT). (10X OBJ.)

Once the image had been made binary it was necessary to erode the image to separate any pollen grains that had been captured. By eroding the image the grains could be separated and therefore captured individually rather than as a collection of pollen grains. This process was done by using the menu function Process - Binary - Options and then selecting a level of erosion that seemed appropriate; this level had to be determined based on what separated the most pollen grains, but still kept the integrity of the image intact. Figure 9 is the result of eroding the binary image above, tape lift 8.36. 

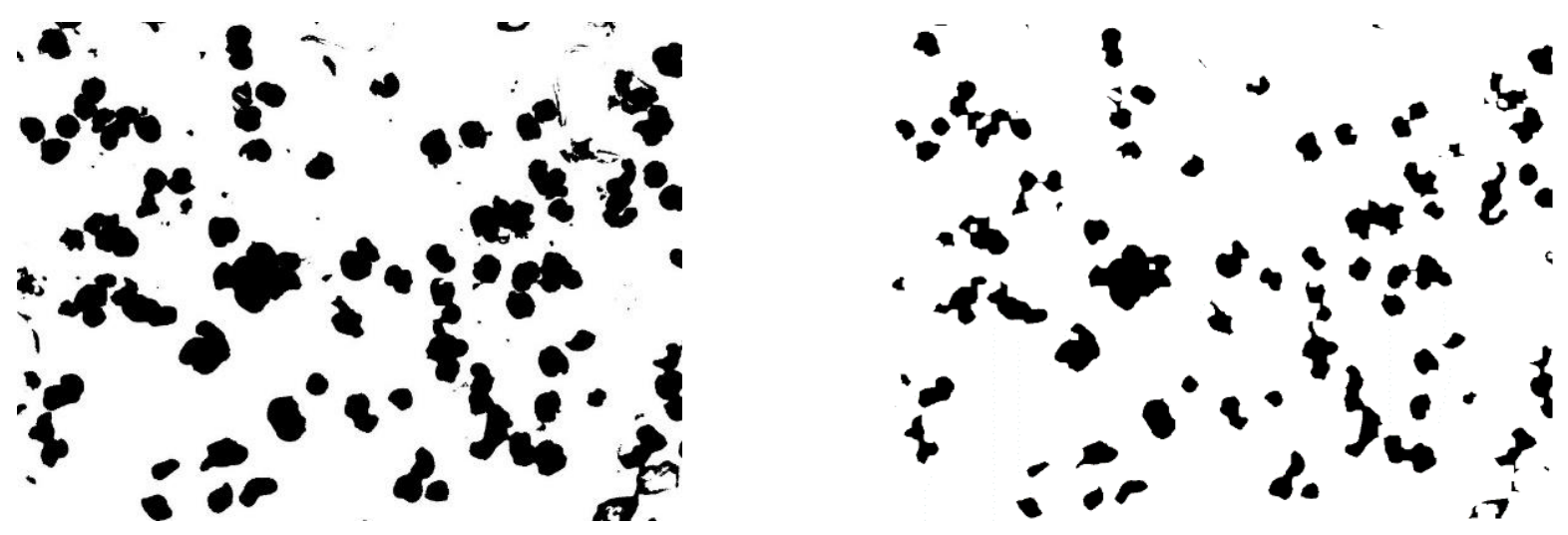

FIGURE 9: PHOTOMICROGRAPHS OF THE BINARY IMAGE OF FRUIT OF THE LOOM T SHIRT VIA TAPE LIFT 8.36 (LEFT) AND ERODED IMAGE OF TAPE LIFT (8.36) (RIGHT). (10X OBJ.)

After the binary image had been eroded the individual pollen grains could be counted. Rather than performing this manipulation by hand on many thousands of images, a macro was used through ImageJ that would automate this process. First, the macro was run on "test images" that were created using Paint; these images had a known number of points. The macro was then used to determine whether or not it gave an accurate count of points. Figure 10 is the counting macro as well as the test images that were used for validation.

var threshold $=30$;

var darkCells = true;

macro "Count and Mark Cells [1]" \{

if (selectionType $<0 \|$ selectionType $>4$ )

exit("Area selection required");

setupUndo;

autoUpdate(false);

if (darkCells) light="light"; else light="'";

run("Find Maxima...", "output=[Point Selection] "+light+" noise="+threshold);

getSelectionCoordinates $(x, y)$;

$n=x$. length;

if (darkCells) setColor("white"); else setColor("black"); 

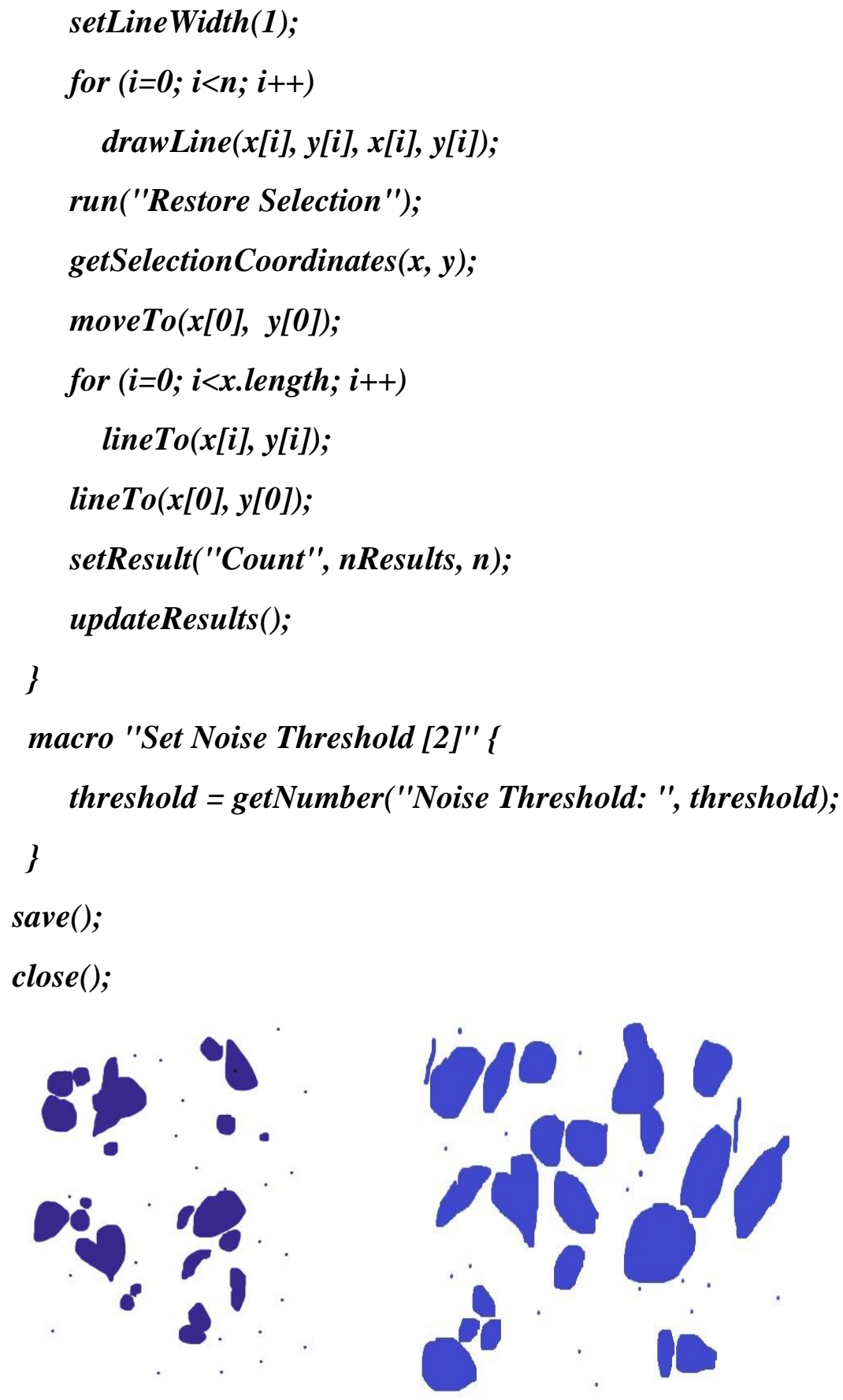

FIGURE 10: "CELL COUNTER" CODE AND EXAMPLES OF VALIDATION IMAGE 1 (LEFT) AND VALIDATION IMAGE 2 (RIGHT) CREATED USING PAINT FOR VALIDATION OF CODE.

After the code had been validated it could be run on all images using the Batch option in ImageJ. 


\section{Statistical Test:}

In order to evaluate whether or not the particular analysis or collection methods are more effective at recovery than another, a two-way ANOVA, or Analysis of Variance, was performed. There are two models that are possible in a two-way ANOVA, the first is the interaction model and the second is the additive model. The interaction model assumes the outcome at one level of analysis (one variable) directly affects the other levels of analysis whereas the additive model assumes that the variables are independent of one another. The additive model was chosen for this study due to the fact that different recovery methods may be more efficient for one type of surface over another, however the results of a tape lift should not affect the results of the vacuum. The coding software called RStudio (Version 0.98.501 - (C) 2009-2013 RStudio, Inc.) was used for all statistical analyses. Post hoc tests are designed for situations in which additional exploration of the differences among means is needed to provide specific information on which means are significantly different from each other. The post hoc test can be run under different conditions, however for this study the Tukey Honest Significant Difference (Tukey HSD) test will be used. Using the Tukey HSD test model of the post hoc test, a pairwise comparison of means is made; Tukey's test is based on a formula very similar to that of the t-test except that it corrects for error rate. It is thus more suitable for multiple comparisons than doing a number of t-tests would be. The Tukey HSD test calculates the minimum distance between means that must be met in order for the two means to be considered significantly different ${ }^{16,17,18,19}$.

In order for ANOVA to be a viable selection for statistical analysis several conditions have to be met. One of these conditions is that the spread of data shall follow a normal distribution, i.e. all data should fall within a bell-shaped curve. The next condition that must be met is that there should be a homogeneity of variance, meaning that the standard deviations of 
the sample means should be uniform with no outliers; the table in Appendix C shows the standard deviations of the data that were used to fit this criteria. As a general rule, the largest variance divided by the smallest should not result is a value larger than two, which was achieved in this research. One last condition for the application of ANOVA is an independence of variables; this is necessary due to the fact that one variable cannot act on the other if a significant difference is to be determined between the two variables. The basic stats package in $\mathrm{R}$ Studio was used to run an ANOVA ${ }^{20}$. The boxplots in Results show the distribution of the mean amounts of pollen recovered using each collection technique on the various surfaces.

\section{Results}

Often times, crime scene investigators and criminalists are unfamiliar with how to process evidence that contains pollen grains. Through this work common recovery methods employed in the field of trace evidence collection were used on different surfaces in order to gauge their efficiency for collecting pollen. Of the recovery methods used the tape lift method was hypothesized to be the method that would collect the greatest amount of pollen most rapidly and with the least amount of disturbance to the surface it was on. 


\section{Amount of Pollen Recovered by Surface}

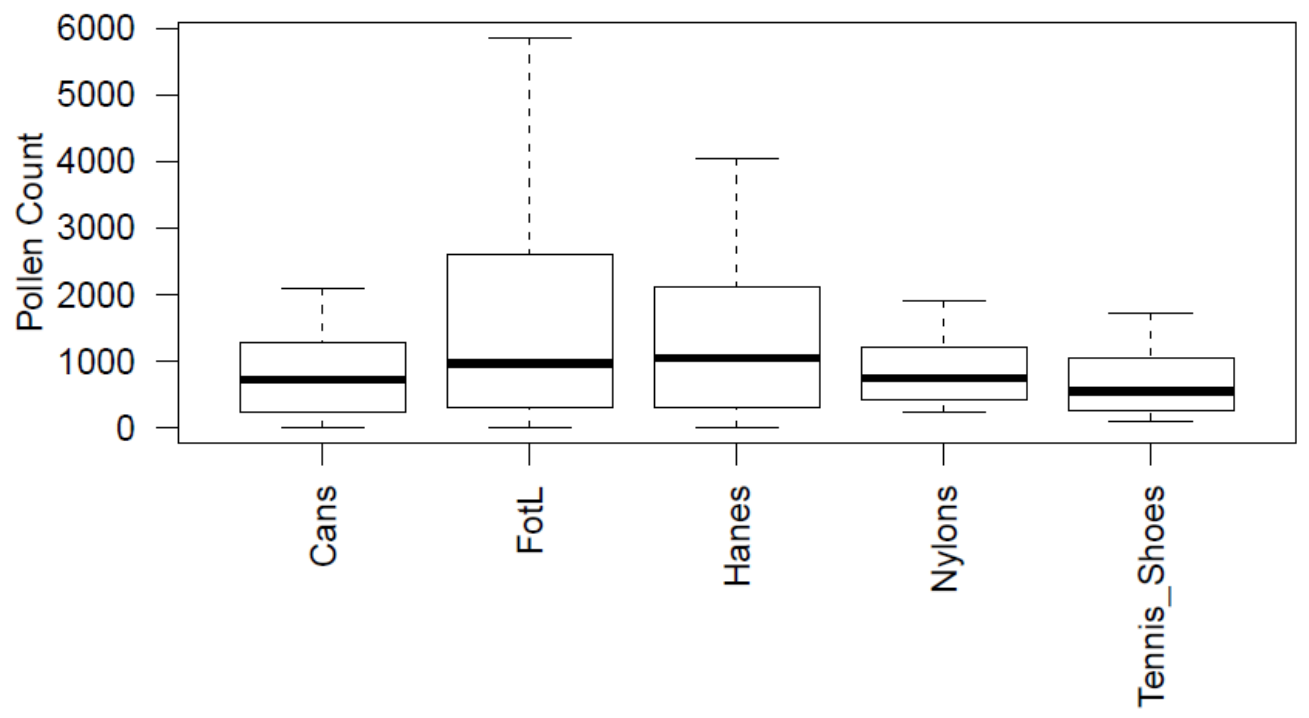

FIGURE 11: BOXPLOT SHOWING THE MEANS OF THE AMOUNT OF POLLEN FOUND ON ALL SURFACES USING THE THREE RECOVERY METHODS (TAPE LIFT, VACUUM, AND SONICATION).

Figure 11 shows the amount of pollen recovered using all three recovery methods on all surfaces.

It appears that some surfaces had more pollen collected than others, however as a whole all surfaces seem to have a similar range of pollen collected. 


\section{Average Amount of Pollen Recovered by Method and Surface}

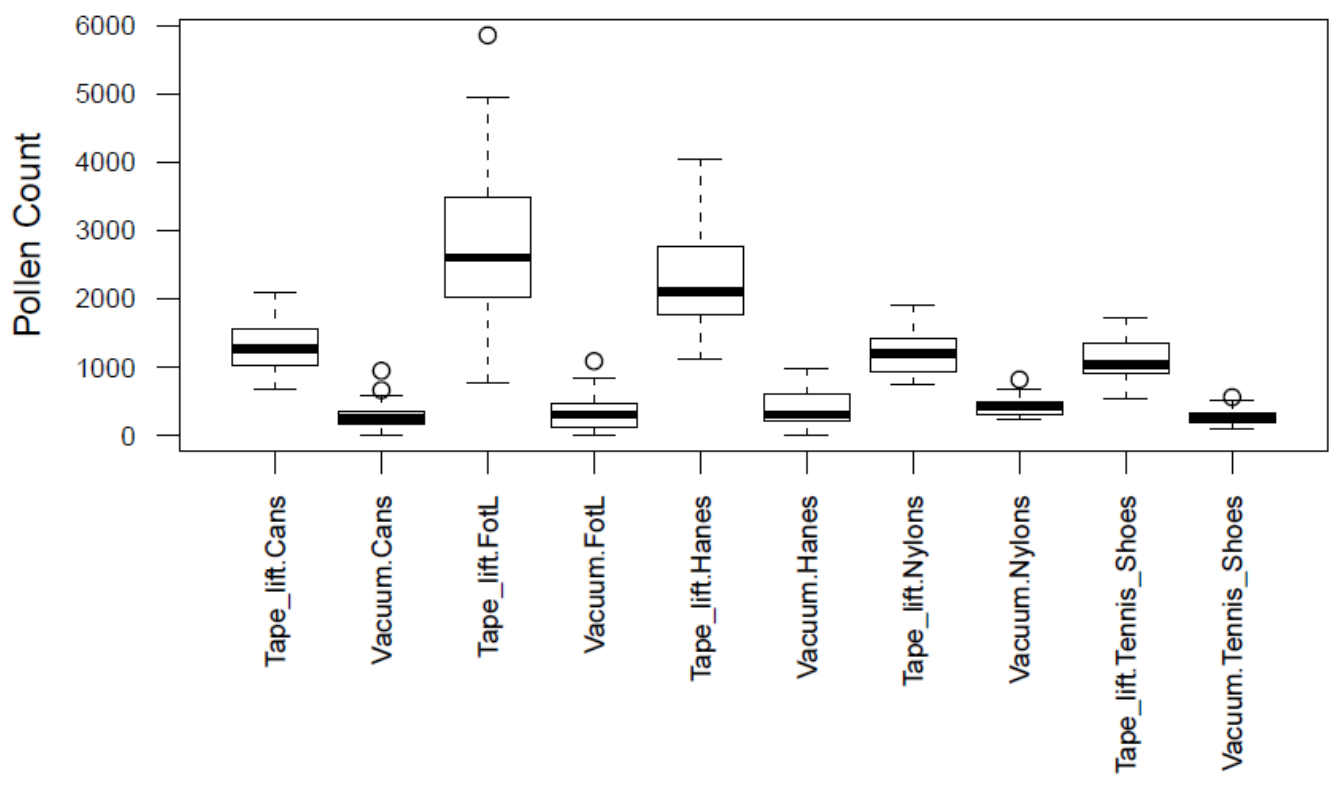

\section{FIGURE 12: BOXPLOT SHOWING THE MEANS OF THE AMOUNT OF POLLEN FOUND ON EACH SURFACE BY EACH RECOVERY METHOD}

Figure 12 represents the amount of pollen recovered from every surface using each of the three recovery methods. 


\section{Amount of Pollen by Method}

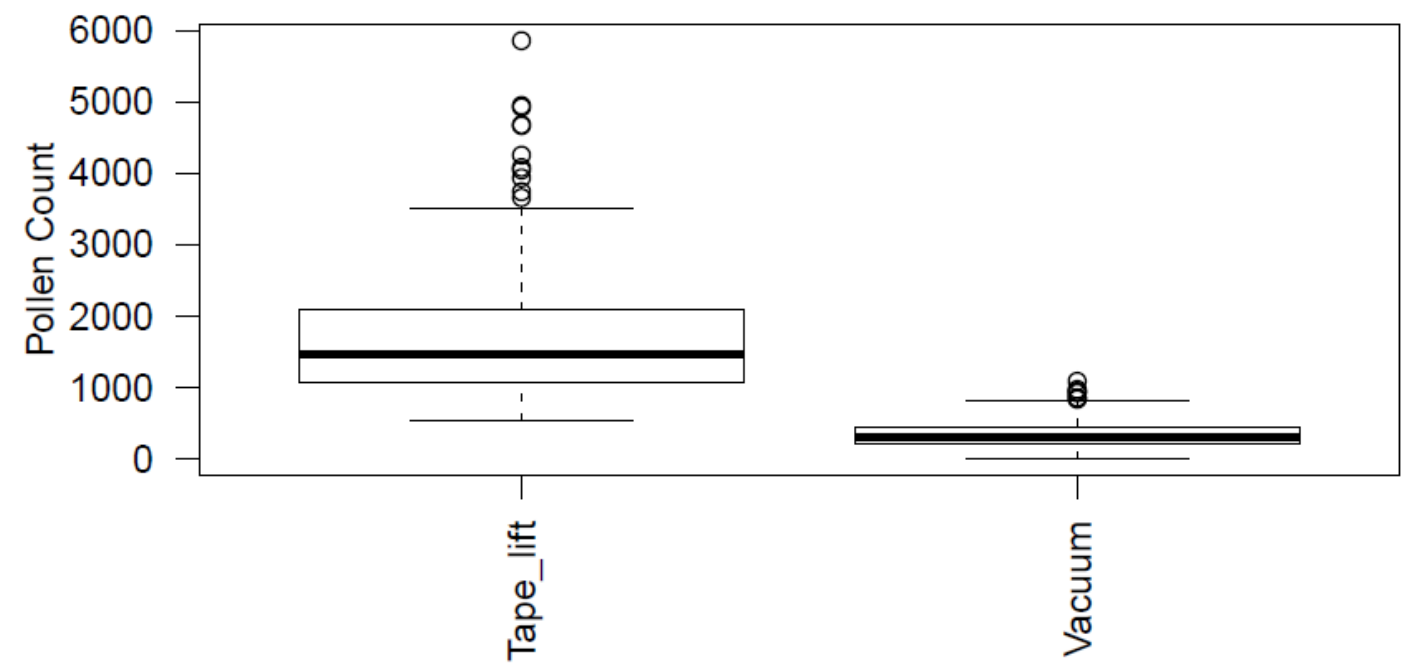

FIGURE 13: BOXPLOT SHOWING THE MEAN AMOUNT OF POLLEN COLLECTED BY EACH METHOD USED ON EVERY SURFACE (TAPE_LIFT AND VACUUM) AS A WHOLE

Figure 13 shows how much pollen was collected using each method that was used for all surfaces. Due to the fact that the sonication method could not be used for the tennis shoes and the metal cans it was removed for the comparison of methods on all surfaces. 
Histogram of Pollen Collected On Fruit of the Loom T Shirts

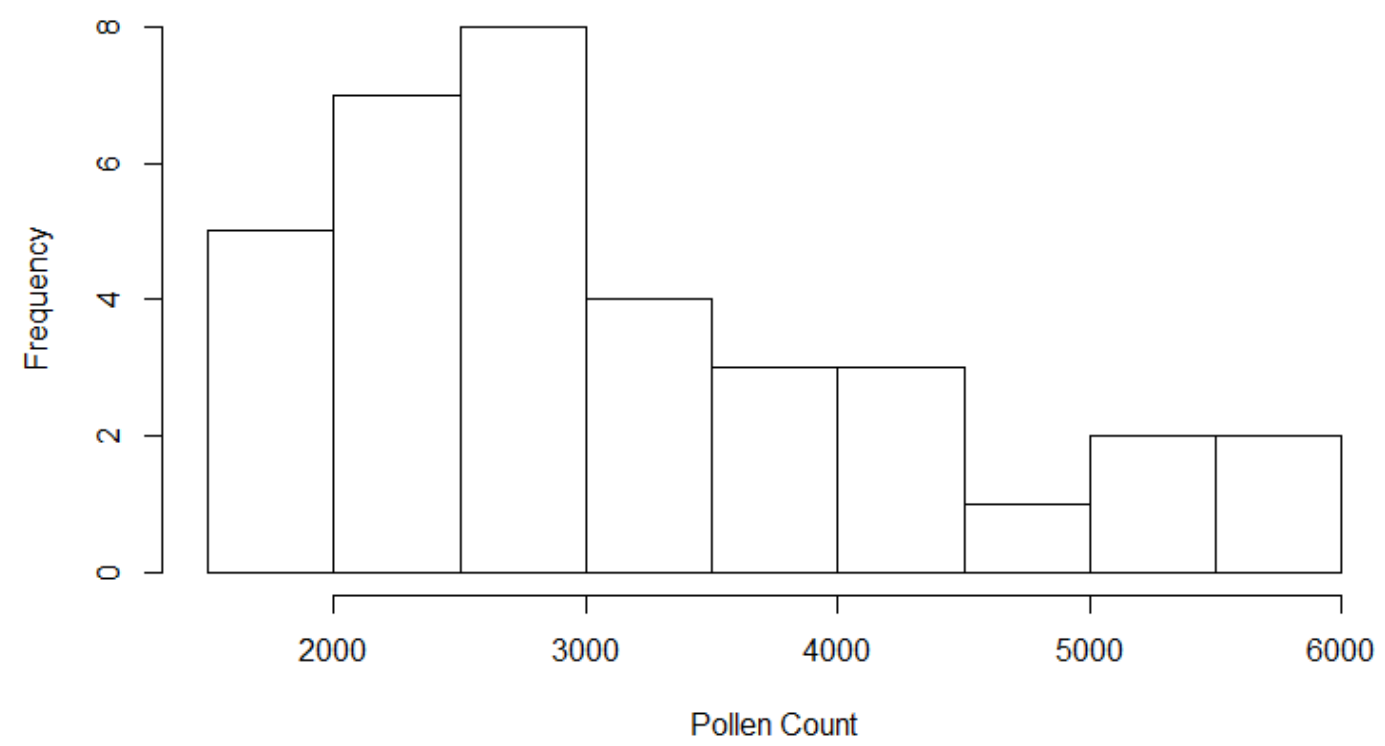

FIGURE 14: HISTOGRAM OF AMOUNTS OF POLLEN COLLECTED FROM FRUIT OF THE LOOM T SHIRTS

Density Curve of Pollen Collected On Fruit of the Loom T Shirts

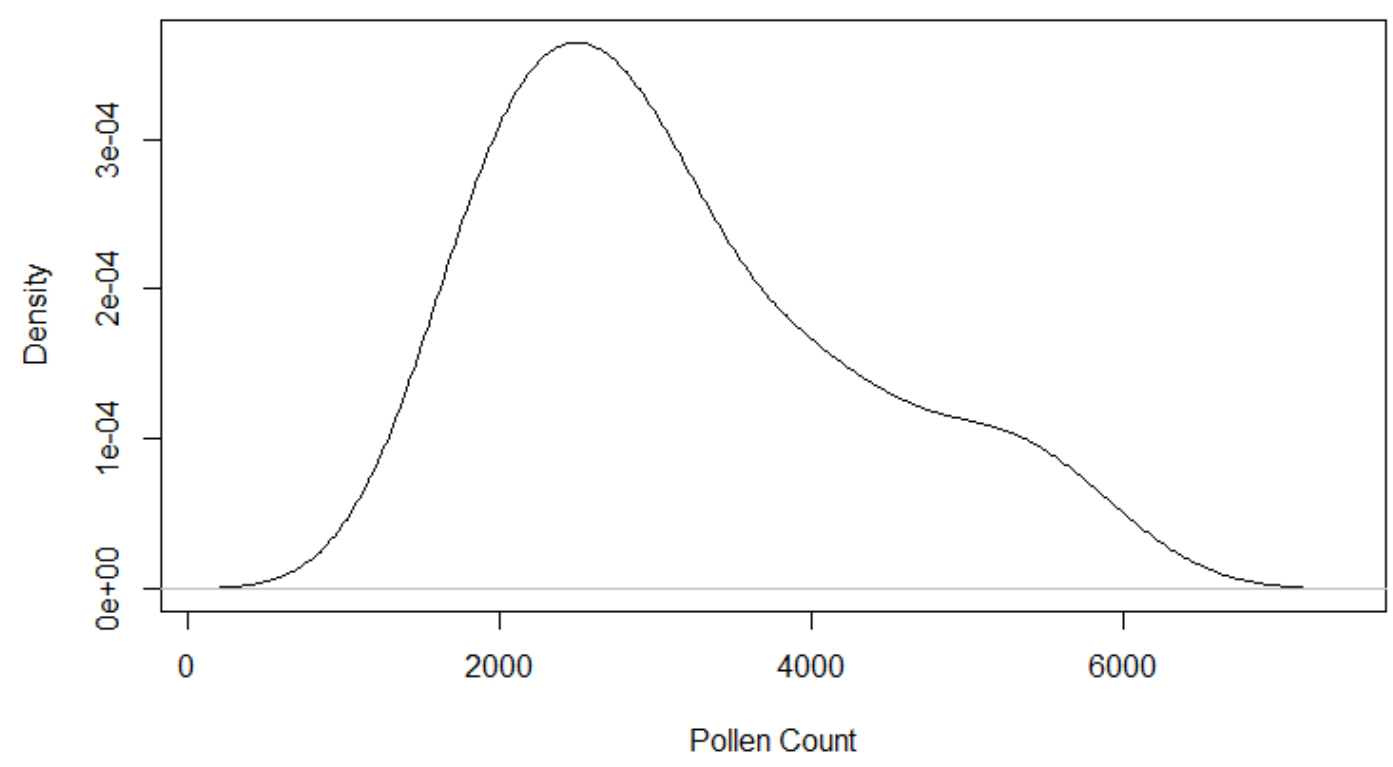

FIGURE 15: DENSITY CURVE ILLUSTRATING POLLEN COLLECTED FROM FRUIT OF THE LOOM T SHIRTS 


\section{Histogram of Pollen Collected On Hanes T Shirts}

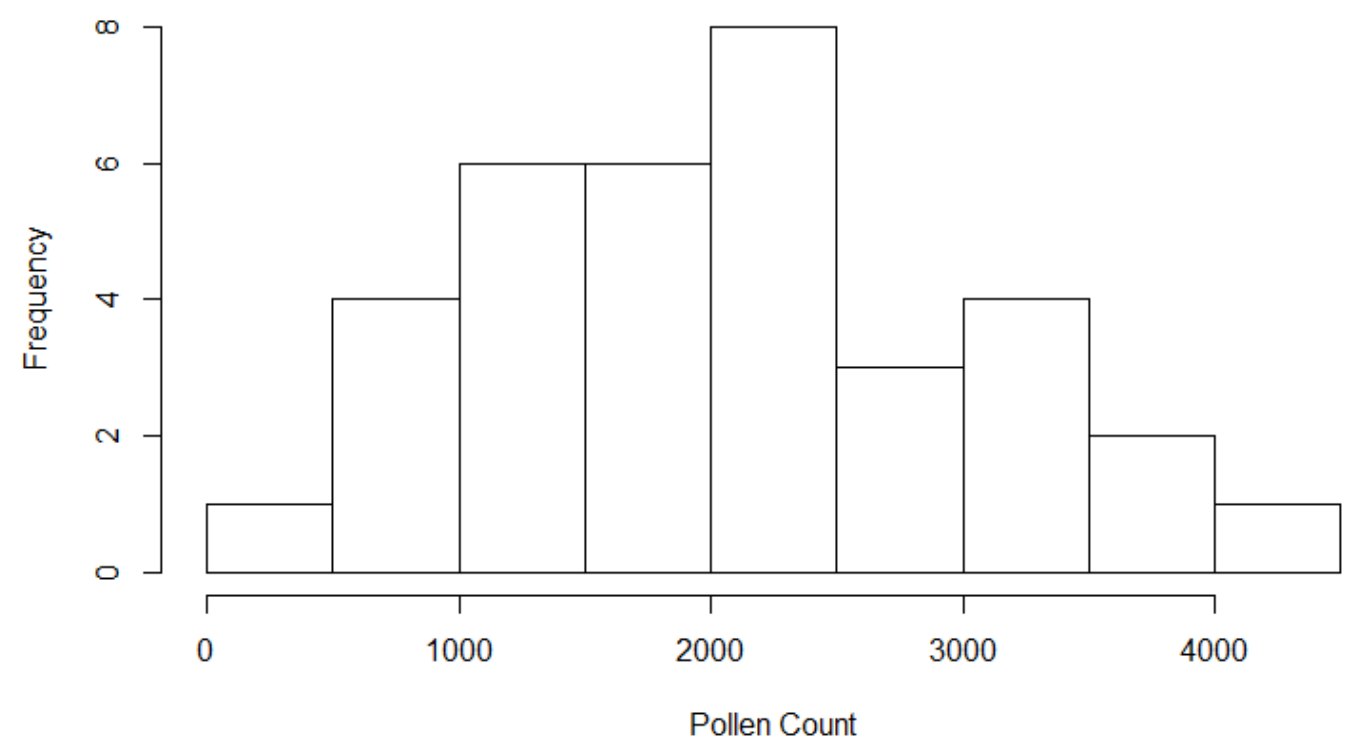

FIGURE 16: HISTOGRAM OF AMOUNTS OF POLLEN COLLECTED FROM HANES T SHIRTS

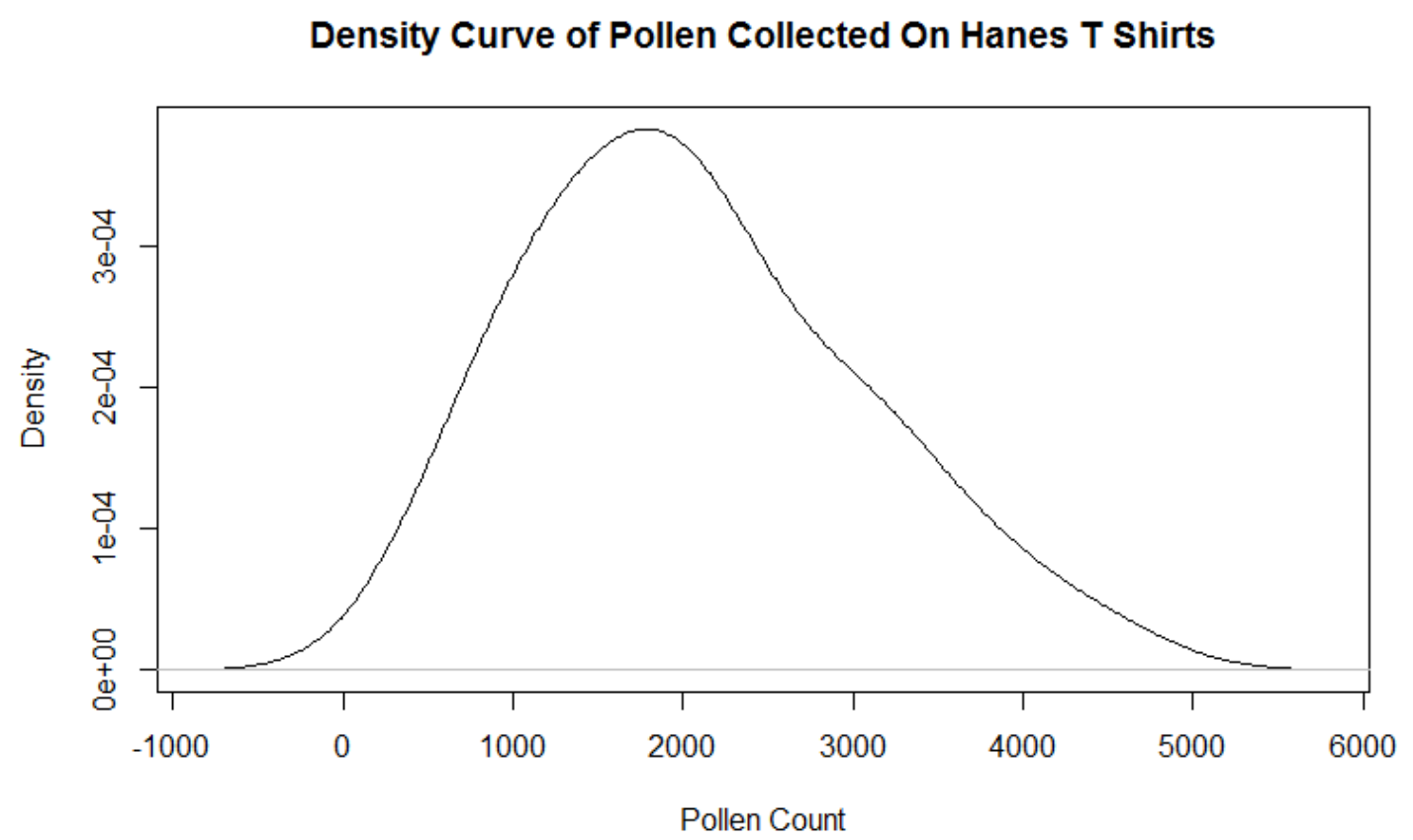

FIGURE 17: DENSITY CURVE ILLUSTRATING POLLEN COLLECTED FROM HANES T SHIRTS 
Histogram of Pollen Collected On Nylons

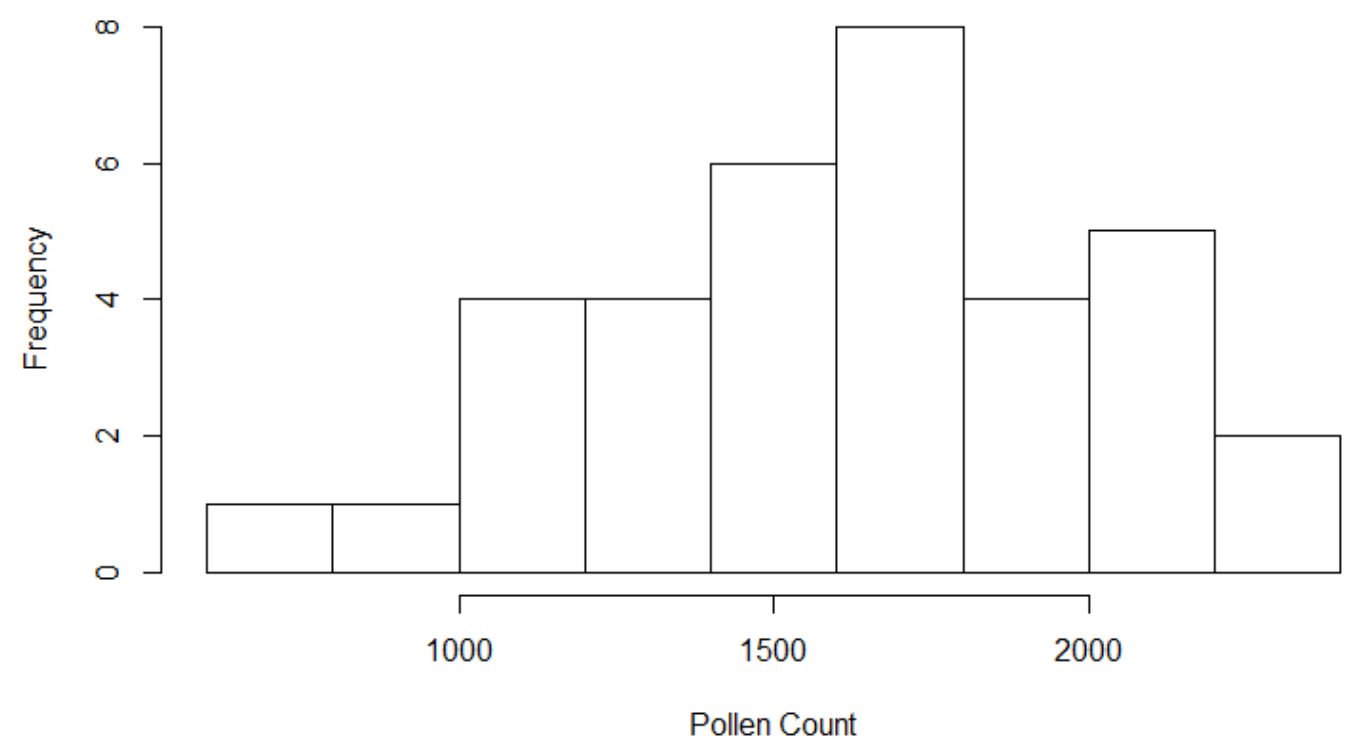

FIGURE 18: HISTOGRAM OF AMOUNTS OF POLLEN COLLECTED FROM NYLONS

Density Curve of Pollen Collected On Nylons

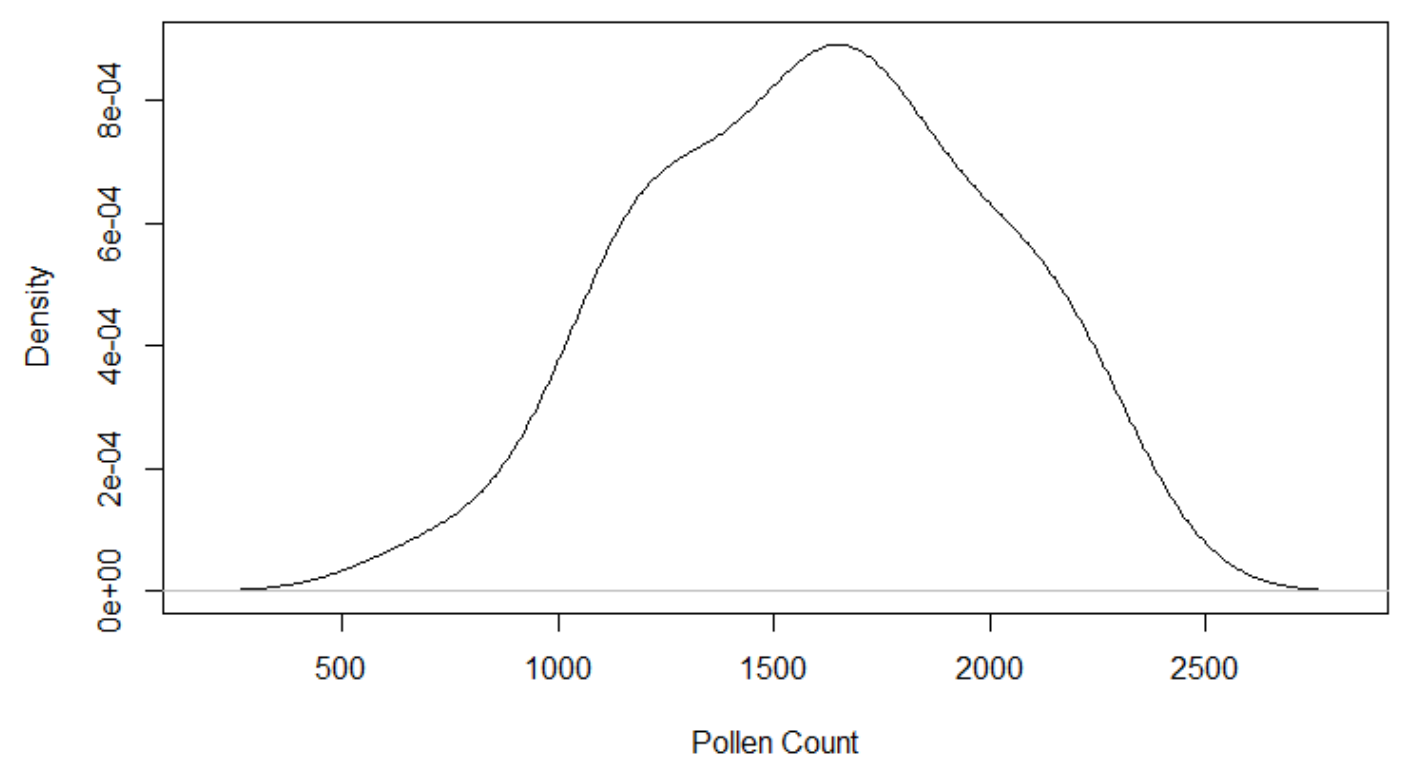

FIGURE 19: DENSITY CURVE ILLUSTRATING POLLEN COLLECTED FROM NYLONS 


\section{Histogram of Pollen Collected On Tennis Shoes}

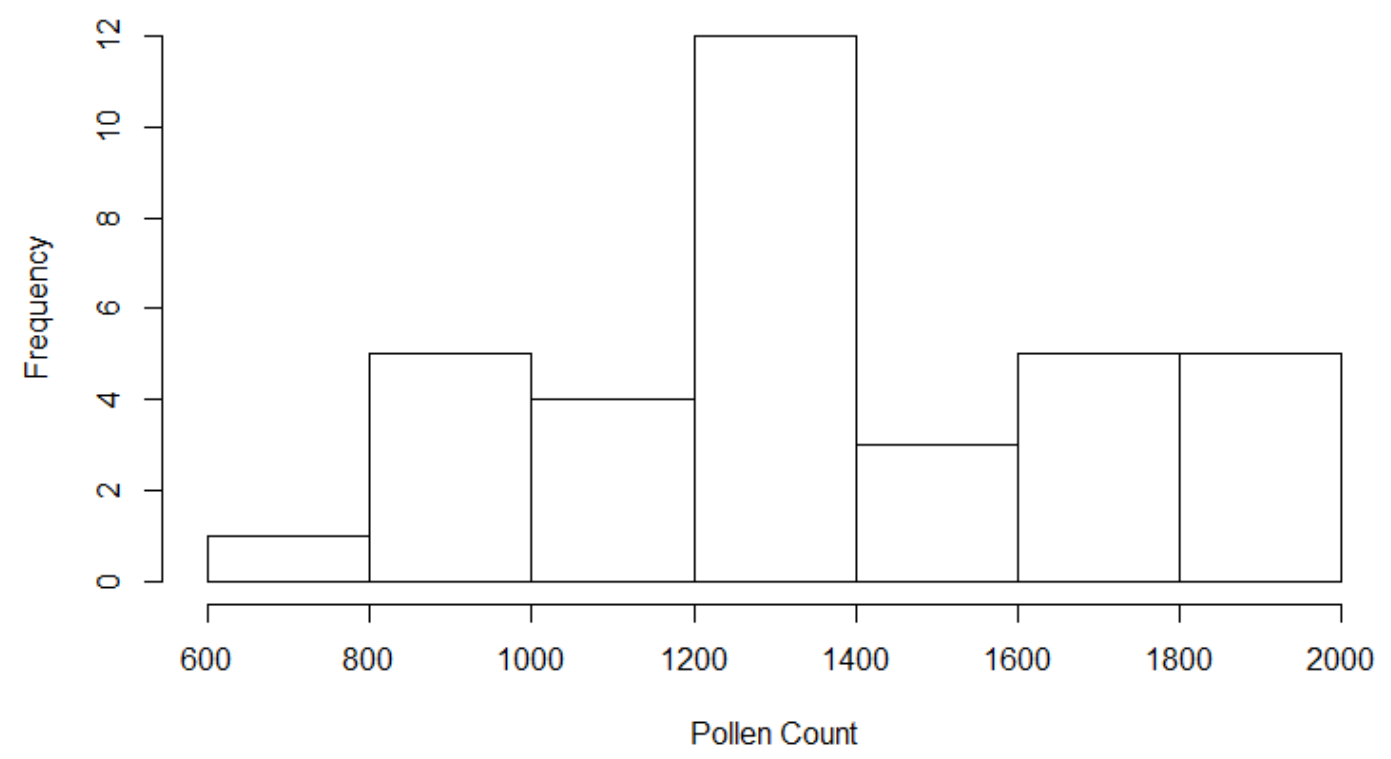

FIGURE 20: HISTOGRAM OF AMOUNTS OF POLLEN COLLECTED FROM TENNIS SHOES

Density Curve of Pollen Collected On Tennis Shoes

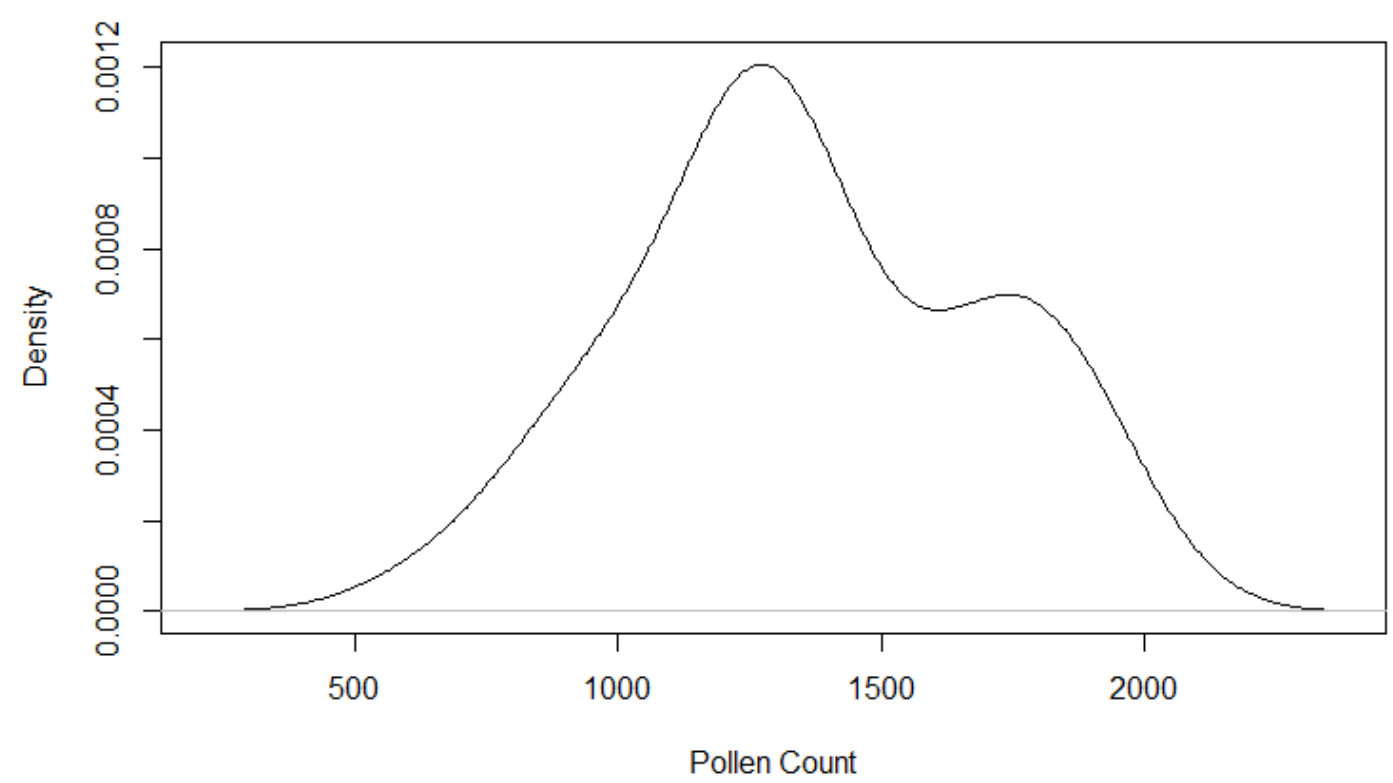

FIGURE 21: DENSITY CURVE ILLUSTRATING POLLEN COLLECTED FROM TENNIS SHOES 
Histogram of Pollen Collected On Cans

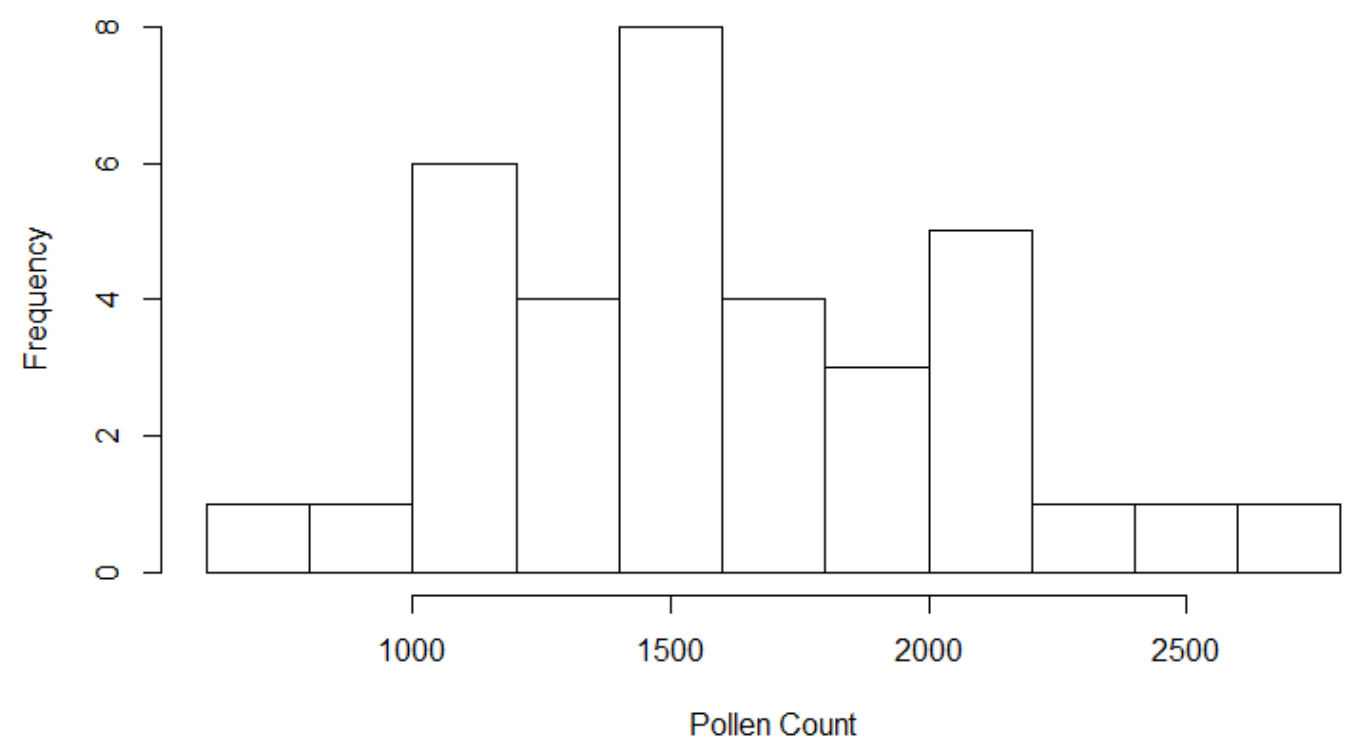

FIGURE 22: HISTOGRAM OF AMOUNTS OF POLLEN COLLECTED FROM CANS

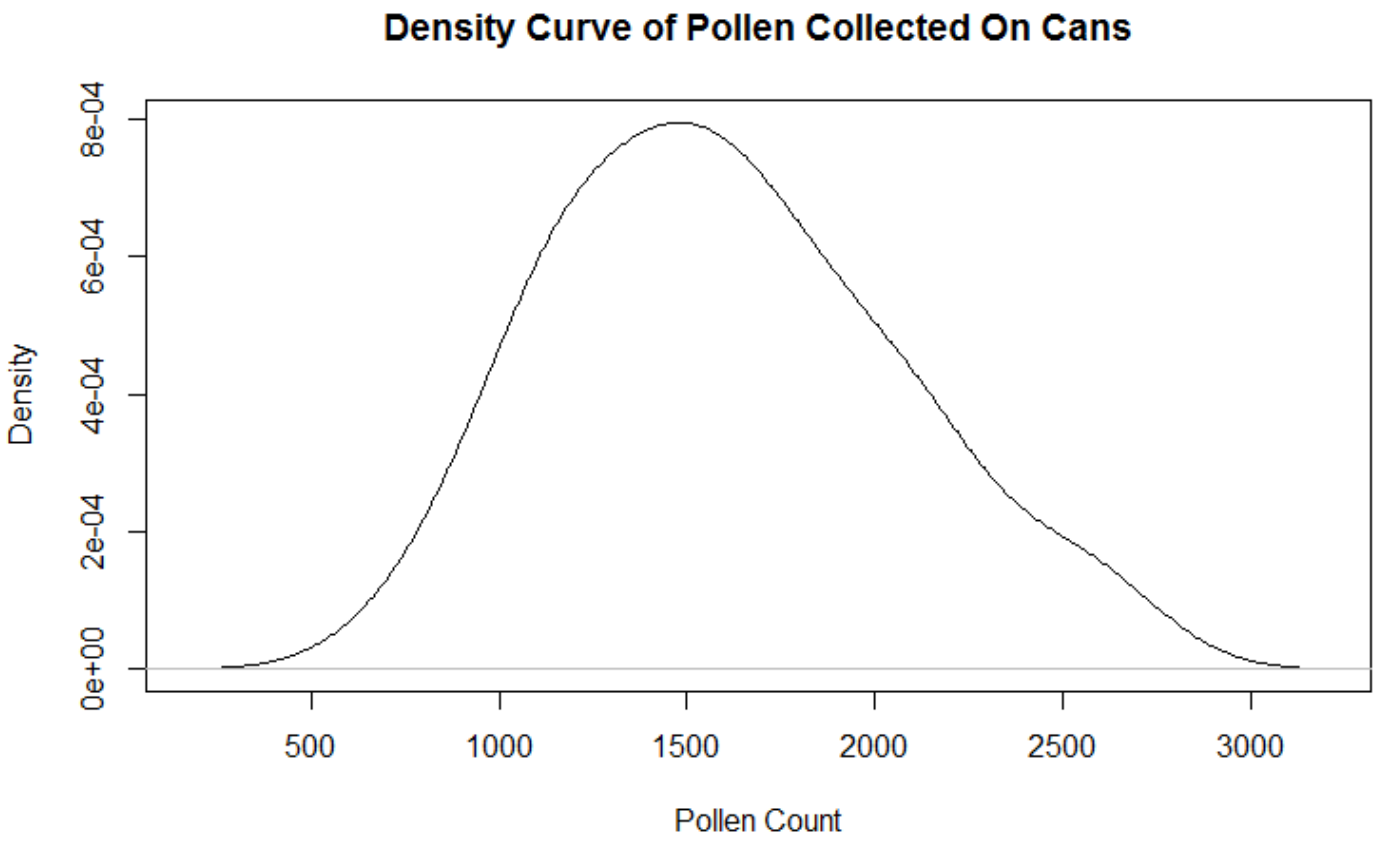

FIGURE 23: DENSITY CURVE ILLUSTRATING POLLEN COLLECTED FROM CANS 
The histograms and density curves above show the spread of the amount of pollen collected from each surface looking at all recovery methods as a whole. This shows that the spread of data is normally distributed and thus ANOVA and Tukey could be performed.

TABLE 3: SUMMARY ANOVA RESULTS FOR AMOUNTS OF POLLEN COLLECTED USING ALL TAPE LIFT AND VACUUM METHODS ON ALL SURFACES

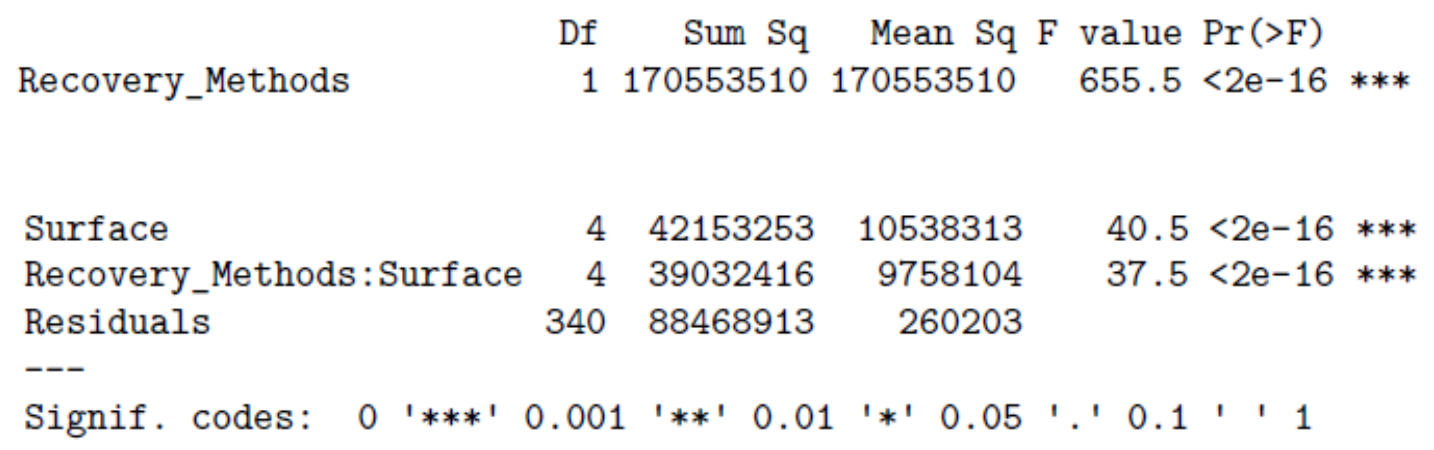




\section{TABLE 4: TUKEY RESULTS FOR ALL RECOVERY METHODS AND SURFACES}

\begin{tabular}{|c|c|c|c|c|c|c|c|c|c|c|c|c|c|}
\hline \\
\hline \multicolumn{14}{|c|}{$\begin{array}{l}\text { \$'Recovery_Methods:Surface` } \\
\text { Control }\end{array}$} \\
\hline & 17 & Vacuum & & Tennis_Shoes & & Vacuum & & Cans & 22.62857 & 410.9858 & 365.7286 & & 1 Same \\
\hline & 371 & Vacuum & & Nylons & & Vacuum & & Hanes & 19.8 & 368.5572 & 408.1572 & & 1 Same \\
\hline & 11 & Vacuum & & FotL & & Vacuum & & Cans & 41.74286 & 346.6143 & 430.1001 & 0.9999989 & 9 Same \\
\hline & 30 & Vacuum & & Tennis_Shoes & & Vacuum & & FotL & 64.37143 & 452.7286 & 323.9858 & 0.9999527 & 7 Same \\
\hline & 26 & Vacuum & & Hanes & & Vacuum & & FotL & 68.68571 & 319.6715 & 457.0429 & 0.9999181 & 1 Same \\
\hline & 28 & Vacuum & & Nylons & & Vacuum & & FotL & 88.48571 & 299.8715 & 476.8429 & 0.9993361 & 1 Same \\
\hline & 41 & Tape_lift & & Tennis_Shoes & & Tape_lift & & Nylons & 97.88571 & 486.2429 & 290.4715 & 0.9985161 & 1 Same \\
\hline & 13 & Vacuum & & Hanes & & Vacuum & & Cans & 110.42857 & 277.9286 & 498.7858 & 0.9962348 & 8 Same \\
\hline & 15 & Vacuum & & Nylons & & Vacuum & & Cans & 130.22857 & 258.1286 & 518.5858 & 0.9873915 & 5 Same \\
\hline & 39 & Vacuum & & Tennis_Shoes & & Vacuum & & Hanes & 133.05714 & 521.4143 & 255.3001 & 0.9853382 & 2 Same \\
\hline & 6 & Tape_lift & & Nylons & & Tape_lift & & Cans & 136.14286 & 524.5001 & 252.2143 & 0.9828098 & 8 Same \\
\hline & 44 & Vacuum & & Tennis_Shoes & & Vacuum & & Nylons & 152.85714 & 541.2143 & 235.5001 & 0.9628232 & 2 Same \\
\hline & 8 & Tape_lift & & Tennis_Shoes & & Tape_lift & & Cans & 234.02857 & 622.3858 & 154.3286 & 0.6559505 & 5 Same \\
\hline & 19 & Tape_lift & & Hanes & & Tape_lift & & FotL & 586.37143 & 974.7286 & 198.0142 & 0.0000986 & 6 Different \\
\hline & 43 & Tape_lift & & Tennis_Shoes & & Vacuum & & Nylons & 677.8 & 289.4428 & 1066.1572 & 0.0000024 & 4 Different \\
\hline & 38 & Tape_lift & & Tennis_Shoes & & Vacuum & & Hanes & 697.6 & 309.2428 & 1085.9572 & 0.000001 & 1 Different \\
\hline & 1 & Vacuum & & Cans & & Tape_lift & & Cans & 1042.05714 & 1430.4143 & 653.6999 & 0 & 0 Different \\
\hline & 2 & Tape_lift & & FotL & & Tape_lift & & Cans & 1493.34286 & 1104.9857 & 1881.7001 & & 0 Different \\
\hline & 3 & Vacuum & & FotL & & Tape_lift & & Cans & 1000.31429 & 1388.6715 & 611.9571 & 0 & 0 Different \\
\hline & 4 & Tape_lift & & Hanes & & Tape_lift & & Cans & 906.97143 & \begin{tabular}{|l}
518.6142 \\
\end{tabular} & 1295.3286 & & 0 Different \\
\hline & 5 & Vacuum & & Hanes & & Tape_lift & & Cans & 931.62857 & 1319.9858 & 543.2714 & & 0 Different \\
\hline & 7 & Vacuum & & Nylons & & Tape_lift & & Cans & 911.82857 & 1300.1858 & 523.4714 & & 0 Different \\
\hline & 9 & Vacuum & & Tennis_Shoes & & Tape_lift & & Cans & 1064.68571 & 1453.0429 & 676.3285 & & 0 Different \\
\hline & 10 & Tape_lift & & FotL & & Vacuum & & Cans & 2535.4 & 2147.0428 & 2923.7572 & 0 & 0 Different \\
\hline & 12 & Tape_lift & & Hanes & & Vacuum & & Cans & 1949.02857 & 1560.6714 & 2337.3858 & & 0 Different \\
\hline & 14 & Tape_lift & & Nylons & & Vacuum & & Cans & 905.91429 & \begin{tabular}{|l|l|} 
& 517.5571 \\
\end{tabular} & 1294.2715 & 0 & 0 Different \\
\hline & 16 & Tape_lift & & Tennis_Shoes & & Vacuum & & Cans & 808.02857 & $\begin{array}{l}419.6714 \\
\end{array}$ & 1196.3858 & & 0 Different \\
\hline & 18 & Vacuum & & FotL & & Tape_lift & & FotL & 2493.65714 & 2882.0143 & 2105.2999 & & 0 Different \\
\hline & 20 & Vacuum & & Hanes & & Tape_lift & & FotL & 2424.97143 & 2813.3286 & 2036.6142 & & 0 Different \\
\hline & 21 & Tape_lift & & Nylons & & Tape_lift & & FotL & 1629.48571 & 2017.8429 & 1241.1285 & & 0 Different \\
\hline & 22 & Vacuum & & Nylons & & Tape_lift & & FotL & 2405.17143 & 2793.5286 & 2016.8142 & & 0 Different \\
\hline & 23 & Tape_lift & & Tennis_Shoes & & Tape_lift & & FotL & 1727.37143 & 2115.7286 & 1339.0142 & & 0 Different \\
\hline & 24 & Vacuum & & Tennis_Shoes & & Tape_lift & & FotL & 2558.02857 & 2946.3858 & 2169.6714 & & 0 Different \\
\hline & 25 & Tape_lift & & Hanes & & Vacuum & & FotL & 1907.28571 & 1518.9285 & 2295.6429 & & 0 Different \\
\hline & 27 & Tape_lift & & Nylons & & Vacuum & & FotL & 864.17143 & \begin{tabular}{|l|l|}
3 & 475.8142 \\
\end{tabular} & 1252.5286 & & 0 Different \\
\hline & 29 & Tape_lift & & Tennis_Shoes & & Vacuum & & FotL & 766.28571 & 377.9285 & 1154.6429 & & 0 Different \\
\hline & 31 & Vacuum & & Hanes & & Tape_lift & & Hanes & 1838.6 & 2226.9572 & 1450.2428 & & 0 Different \\
\hline & 32 & Tape_lift & & Nylons & & Tape_lift & & Hanes & 1043.11429 & 1431.4715 & 654.7571 & & 0 Different \\
\hline & 33 & Vacuum & & Nylons & & Tape_lift & & Hanes & 1818.8 & 2207.1572 & 1430.4428 & & 0 Different \\
\hline & 34 & Tape_lift & & Tennis_Shoes & & Tape_lift & & Hanes & 1141 & 1529.3572 & 752.6428 & & 0 Different \\
\hline & 35 & Vacuum & & Tennis_Shoes & & Tape_lift & & Hanes & 1971.65714 & 2360.0143 & 1583.2999 & & 0 Different \\
\hline & 36 & Tape_lift & & Nylons & & Vacuum & & Hanes & 795.48571 & 407.1285 & 1183.8429 & 0 & 0 Different \\
\hline & 40 & Vacuum & & Nylons & & Tape_lift & & Nylons & 775.68571 & 1164.0429 & 387.3285 & & 0 Different \\
\hline & 42 & Vacuum & & Tennis_Shoes & & Tape_lift & & Nylons & 928.54286 & 1316.9001 & 540.1857 & & 0 Different \\
\hline & 45 & Vacuum & & Tennis_Shoes & & Tape_lift & & Tennis_Shoes & 830.65714 & 1219.0143 & 442.2999 & & 0 Different \\
\hline
\end{tabular}

Table 4 shows the results of performing a Tukey test on the mean amounts of pollen collected for

all surfaces using the tape lift method and the vacuum method. From that it can be seen which

means were established to be the same and which means were found to have a significant 
difference. Figure 24 depicts a graphical representation of these findings. In any instance where the " 0 " mark on the $\mathrm{x}$-axis is crossed the means are statistically the same, whereas in all other areas there is a significant difference between the means.

\section{$95 \%$ family-wise confidence level}
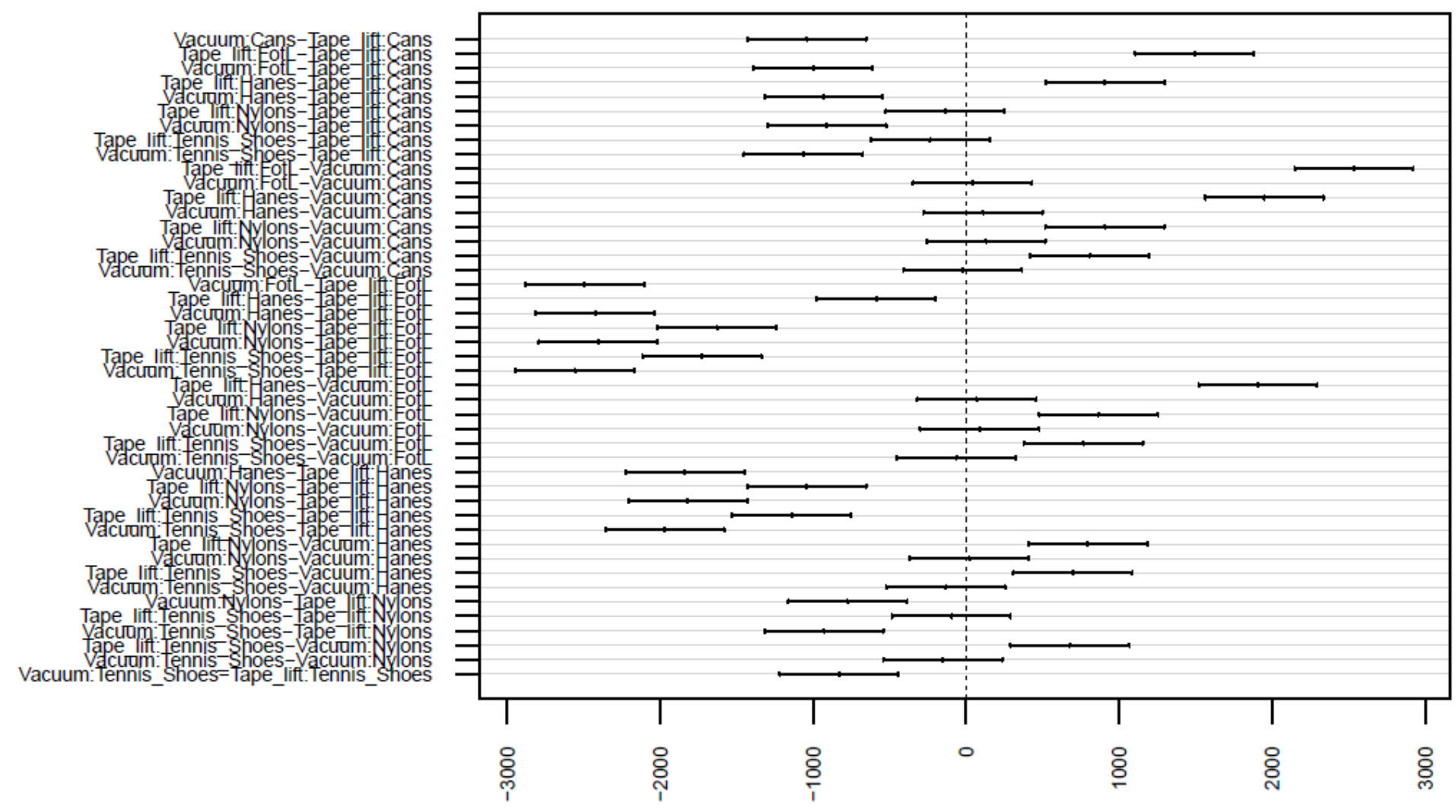

Differences in mean levels of Recovery_Methods:Surface

FIGURE 24: GRAPHICAL REPRESENTATION OF TUKEY RESULTS OBTAINED FOR COMPARISON OF MEANS BETWEEN ALL SURFACES USING THE TAPE LIFT AND VACUUM RECOVERY METHODS 


\section{$95 \%$ family-wise confidence level}

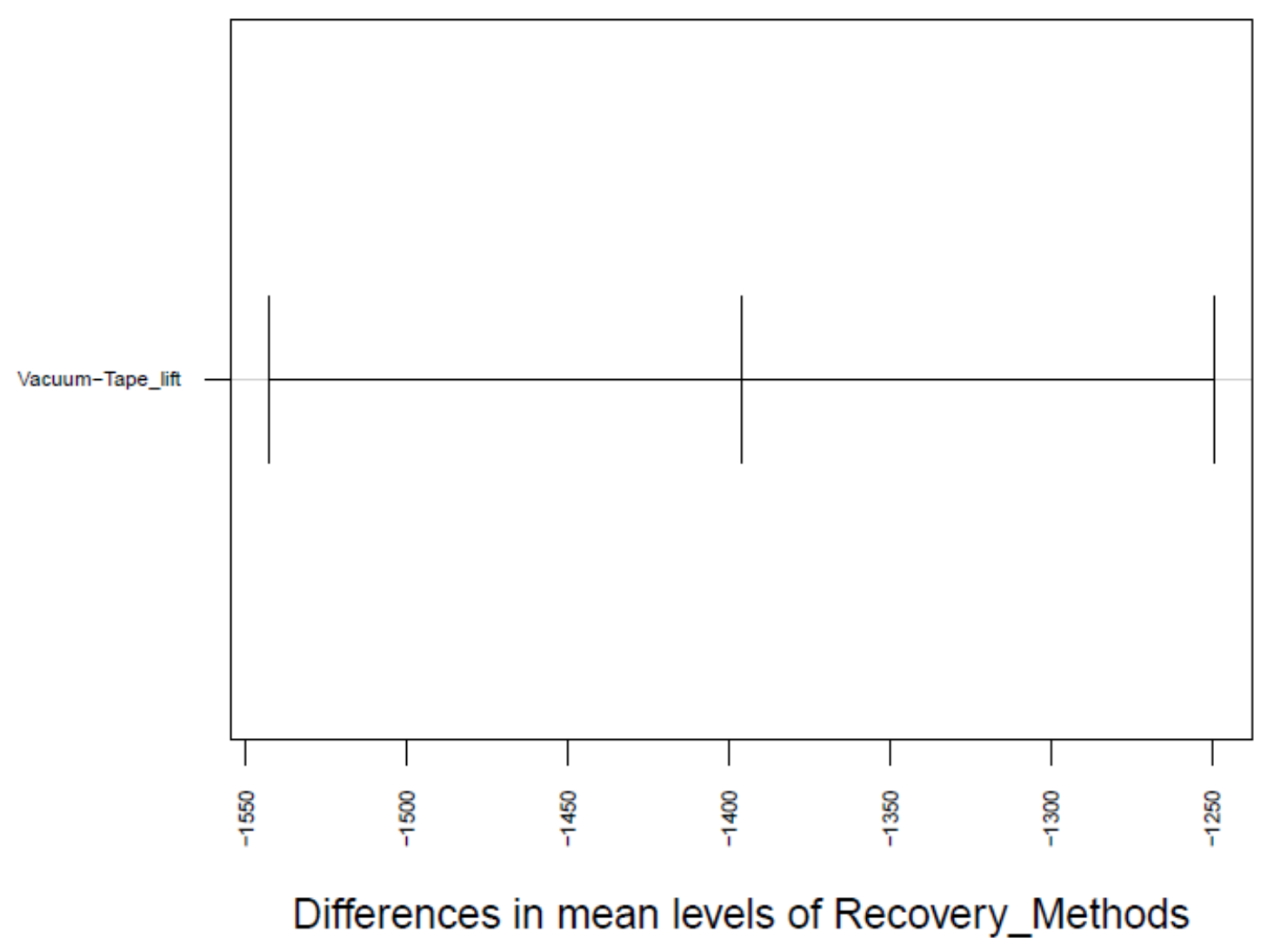

FIGURE 25: GRAPHICAL REPRESENTATION OF TUKEY RESULTS FOR THE MEANS OF RECOVERY METHODS 


\section{$95 \%$ family-wise confidence level}

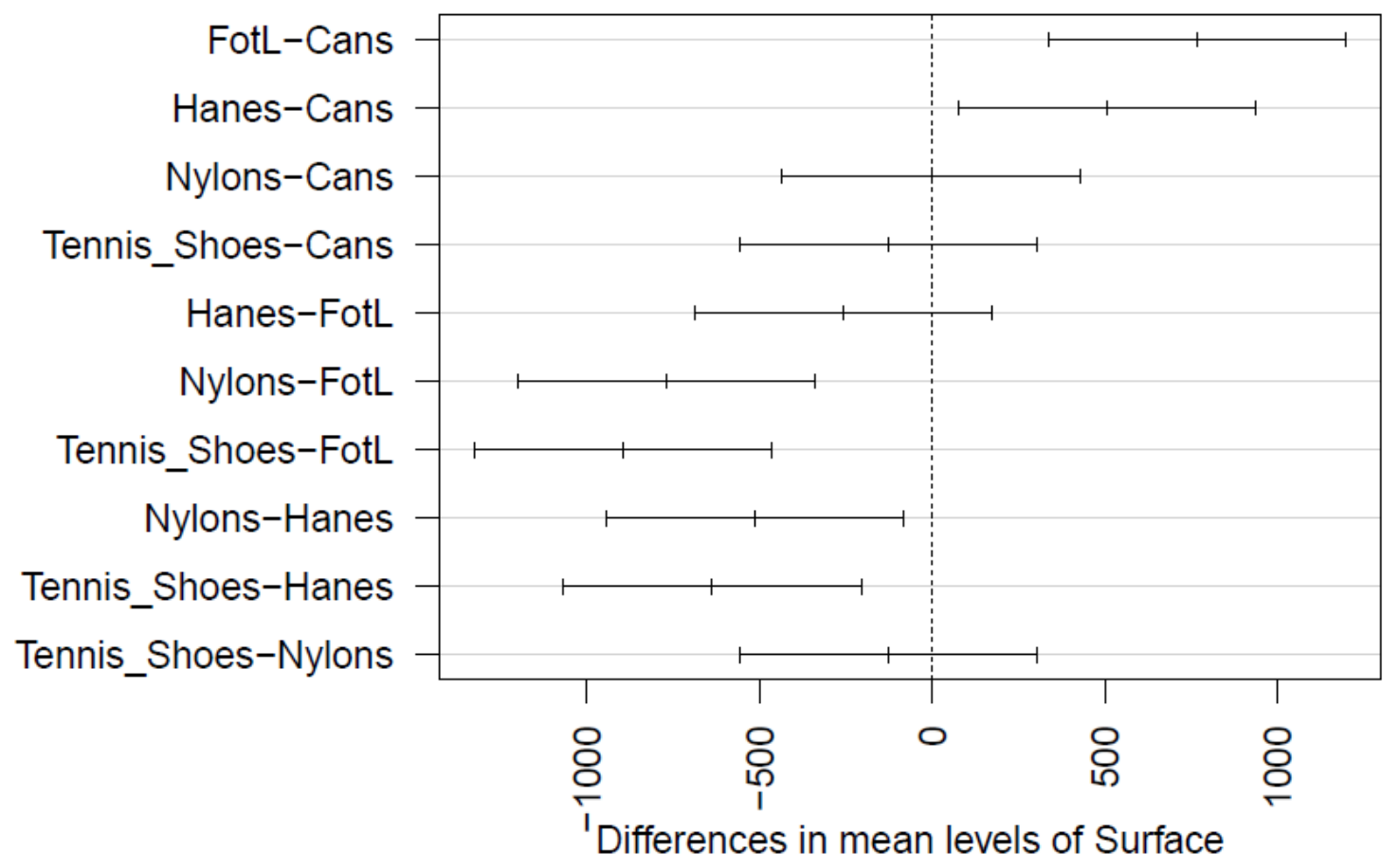

FIGURE 26: GRAPHICAL REPRESENTATION OF TUKEY RESULTS FOR MEANS OF SURFACES

\section{Discussion}

The use of trace evidence recovery methods for materials such as hairs, fibers, glass, and paint chips has been well established within the forensic science community, however within the scope of analyzing pollen and soil as evidence the applications of tape lifting, vacuuming, and sonication are relatively unknown. In this study, the use of these methods was tested on two brands of cotton $\mathrm{t}$ shirts, $100 \%$ nylon stockings, metal cans, and tennis shoes in order to determine effectiveness of the methods. Based on all results, as well as the boxplots, histograms, and density curves shown in Figure 11 - Figure 23, it can be said that the tape lift method was most effective at collecting the greatest amount of test dust. The Fruit of the Loom t shirts (Figure 142) were the only surface that exhibited a substantial difference with the other surfaces 
in terms of the amount of pollen that was collected. The difference the two brands of $t$ shirts was unexpected, however one possible explanation as to why this was observed may be the way in which the cotton was woven together which may allow for the pollen to be collected more readily off of the Fruit of the Loom brand of $t$ shirt. The density curve below shows the amounts of pollen collected as a whole off of both $t$ shirts and while they are similar, the range of pollen collected for the Fruit of the Loom $t$ shirt is greater than that of Hanes, therefore this may explain why their means were found to significantly different after Tukey was performed.

\section{Density of Pollen Count by Tape Lift Method}

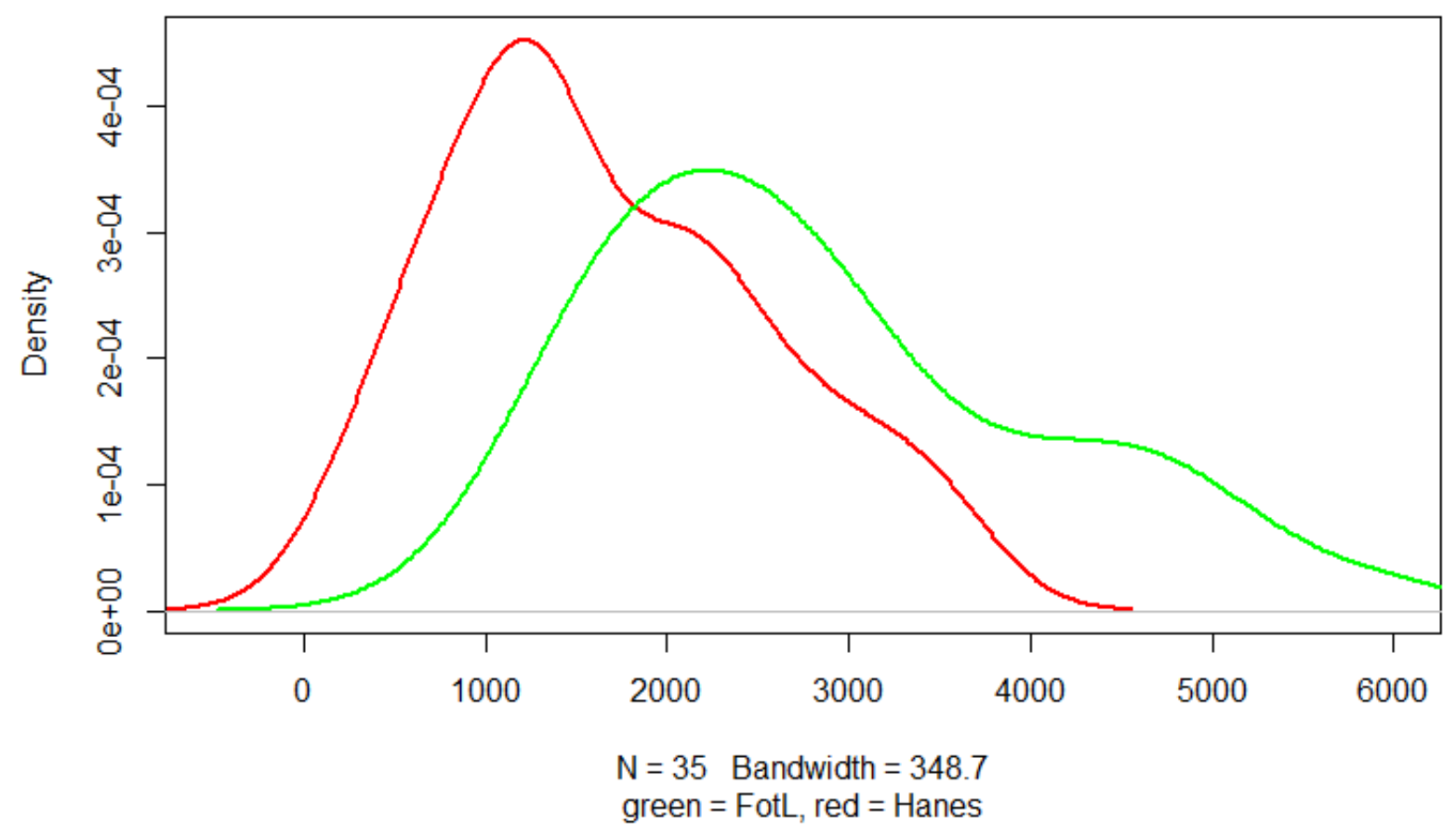

FIGURE 27: DENSITY CURVE FOR THE AMOUNTS OF POLLEN COLLECTED USING THE TAPE LIFT METHOD FOR BOTH BRANDS OF T SHIRTS

The tennis shoes and the metal cans follow the trend of having the greatest amount of pollen collected using the tape lift method, however it was observed that as a whole the two surfaces had the least amount of pollen collected with all methods employed as compared to the $t$ shirts and the nylon stockings. Upon examination this results seems logical because the pollen 
grains would not have fibers from fabric to become embedded in, therefore when the test dust was deposited on the surfaces a great deal may not have adhered. The tennis shoes and metal cans may also have exhibited lower pollen count results due to the nature of their surfaces, meaning the grooves of the cans and the treads of the tennis shoes. The pollen was found to concentrate in the indented areas and while the tape and the vacuum were able to recover a great deal it was observed that these areas had more pollen remaining after the recovery methods were used than any of the cloth samples.

With all of the surfaces used it was seen that the tape lift method was most effective. This result seems logical because it is the method that makes the most direct contact with the various surfaces. The vacuum filtration method was the next in terms of how much pollen was collected, however it was observed during experimentation that it was quite easy for the pollen to pass by the filter paper and go down the vacuum hose instead, however it should be noted that the vacuum system used in this research was of low quality and in casework a higher quality system would be employed.

The least amount of pollen was recovered using the sonication method, with the exception of the Fruit of the Loom shirts. However, overall the sonication method did perform the worst. This result was expected due to the numerous issues that arose while performing that particular recovery. Each surface was run through two cycles using the sonicator, however there was still a great amount of test dust remaining on the surface. Moreover, the test dust that had settled in to the water had to be run through a piece of filter paper, which would also serve as an area of loss if the filter paper did not catch all of the pollen or if any pollen remained in the sonicator even though it was not visually observed. The sonication samples could not be viewed under the fluorescence microscope and had to have a weight conversion performed. 
The results of the Tukey test show that the significant differences in recovery methods lie between the tape lift method and the vacuum method. Due to the fact that the sonication method was not used during the statistical analysis the results for that method were not used during the comparisons. The $\mathrm{p}$ adj. value for the recovery methods was 0 with no means overlapping zero on the $\mathrm{x}$-axis (Figure 25) indicating that the two methods are significantly different from one another. The results of the Tukey test run on the surfaces alone indicate that the differences lie between all surfaces excluding Nylons: Cans, Tennis Shoes: Cans, Hanes: Fruit of the Loom, and Tennis Shoes: Nylons. These surfaces have means overlapping zero which indicate that they are statistically the same.

The ESDA aerosol development hood was used to deposit the test dust on all surfaces in order to mimic the wind blowing pollen onto a certain object. This was a novel approach in the area of evaluating method efficacy and the results proved to be quite promising. All surfaces that were placed under the chamber had an even layer of test dust deposited on them and the air circulation throughout the chamber allowed for sufficient reproducibility between trials. One drawback in the use of the ESDA hood was that the area covered by the chamber was larger than the objects that were inside, therefore there was some test dust lost to the surrounding areas and not on the desired surface due to the inherent ability of the circulation fan to deposit dust evenly throughout the entire chamber. The test dust loss could perhaps be decreased by placing two or more of the objects in the chamber simultaneously, however having multiple objects in the chamber at once would affect knowing exactly how much test dust was being deposited on the surface.

In this study effectiveness, which was defined as the method that would recover the greatest amount of pollen from each surface, while at the same time allowing for visualization 
and manipulation of the pollen grains after they had been collected, was used as the main measurement tool for evaluating which recovery method was most practical for use in cases of pollen evidence. The tape lift method was determined to be the most effective because it collected the most pollen, was able to be viewed easily directly under the microscope, and if necessary the pollen could easily be removed from the tape and processed further in a casework scenario. To determine effectiveness all surfaces had to be relatively consistent in size, therefore all cloth samples (both brands of $\mathrm{t}$ shirts as well as nylon stockings) were cut to be roughly 6" $\mathrm{x}$ 6" squares. The metal cans were approximately the same size as well and the tennis shoes ranged from women's size 6 wide to size 11 wide. Due to fact that 35 pairs of shoes had to be purchases it was not possible to have all the same size, however on the larger shoes the toe and heel regions were excluded from collection of pollen in an effort to maintain consistency in the area that the particular recovery method covered, however this may be a limit of the efficacy measure.

ImageJ was used during image analysis in order to not only count the pollen grains, but to separate the grains as much as possible so that they could be individually assessed rather than as a cluster of points. This method showed extremely promising results as to how image analysis of pollen could be performed and as a whole most pollen points were able to be counted singularly, which allowed for an accurate count of the pollen captured in one photograph. The use of ImageJ also provided a rapid approach to counting thousands of pollen grains and through validation with test images this is believed to be an exceptional method for automating an otherwise monotonous task. By automating the counting process there would be less room for human error in counting, especially if there were a similar case where thousands of points needed 
to be counted. One pitfall of this method was the fact that all images had to be converted in to binary images. Therefore, taking images under the various UV filters became rather obsolete, which did not allow for determination of whether or not the recovery method collected the pollen grains and the calcite equally. All of these factors also serve as limitations in determining effectiveness, though it can be said based on the relative success of all other facets of this research that the effectiveness determination was achieved.

\section{Conclusions/Future Research}

From the results of this research, the crime scene investigators and criminalists are able to see that the tape lift method is the superior choice for how pollen evidence should be collected. This method showed the greatest recovery rate and worked well with all of the chosen surfaces. In the future different pollen types may be explored in order to determine whether or not the type of pollen also had an effect on the amount of test dust recovered from surfaces or on the effectiveness of the tape lift method. Pollen grains have different structures depending on the species, therefore it may be useful to know if this would affect the chosen recovery method. The use of the ESDA hood for depositing the test dust on the surfaces was a novel approach in the field of forensics and showed promising results, however through further work it may be possible to decrease the amount of loss to the environment and obtain a more reliable result for the exact amount of test dust on the surfaces. The application of image erosion through ImageJ as a means of pollen grain counting was also found to be successful and through further calibration and testing full automation of point counting may be possible not only in pollen analysis as evidence, but as well as in other disciplines within forensics. 


\section{References}

[1] Drori, J. TED.

https://www.ted.com/talks/jonathan_drori_every_pollen_grain_has_a_story?language=en (accessed Nov 10, 2015).

[2] Horrocks, M, Walsh, K. Fine Resolution of Pollen Patterns in Limited Space: Differentiating a Crime Scene and an Alibi Scene Seven Meters Apart. Journal of Forensic Science 44.2 (1999): 417-20.

[3] Bisbing, R.; Mute Witness: Trace Evidence Analysis. Chapter 5 Finding Trace Evidence. (2001): 102

[4] Chable, J., Roux, C., and Lennard, C., Collection of Fiber Evidence Using Water-Soluble Cellophane Tape. Journal of Forensic Sciences, JFSCA, Vol. 39, No. 6, November 1994, pp. $1520-1527$.

[5] Kirk, P.L, Microscopic Evidence--Its Use in the Investigation of Crime, 40 J. Crim. L. \& Criminology 362 (1949-1950)

[6] Ruffell, A., Sandiford, A. Maximizing Trace Soil Evidence: An Improved Recovery Method Developed during Investigation of a \$26 Million Bank Robbery. Forensic Science International 209.1-3 (2011): E1-E7.

[7] Angiosperms vs Gymnosperms. Diffen.com. Diffen LLC, Web. 15 May 2014.

< http://www.diffen.com/difference/Angiosperms_vs_Gymnosperms >

[8] Erdtman, G. Pollen Morphology and Plant Taxonomy: Angiosperms. Contacts of Palynology; (1986): 3 
[9] Horrocks, M, Walsh, K. Fine Resolution of Pollen Patterns in Limited Space: Differentiating a Crime Scene and an Alibi Scene Seven Meters Apart. Journal of Forensic Science 44.2 (1999): $417-20$.

[10] Horner. Air and Dust borne Mycoflora in Houses Free of Water Damage and Fungal Growth. Applied and Environmental Microbiology, Vol. 70, No. 11; 6394-6400 [11] Pollen Library (Plants That Cause Allergies). List of Top Pollen Offenders.

[12] Peterson, R., Slovin, J.P., and Changbin C. A Simplified Method for Differential Staining of Aborted and Non-aborted Pollen Grains. International Journal of Plant Biology 1.2 (2010): 1-5.

[13] "Scientific Working Group for Material Analysis." Scientific Working Group for Material Analysis.

[14] "SWGTREAD - Home." SWGTREAD - Home.

[15] Ruffell, A., Sandiford, A. Maximizing Trace Soil Evidence: An Improved Recovery Method Developed during Investigation of a \$26 Million Bank Robbery. Forensic Science International 209.1-3 (2011): E1-E7.

[16] McDonald, J. Chapter 11: Two-Way ANOVA. Carnegie Mellon University,

[17] How To Run Statistical Tests in Excel. Science \& Justice. CBGS M\&E Science.

[18] Stevens; "Post hoc Tests in ANOVA", (1999) 1-4

[19] "The Collected Works of John W. Tukey"; David Brillinger; 1984

[20] Package: stats, Version: 3.0.2, Priority: base, Title: The R Stats Package, Author: R Core Team and contributors worldwide, Maintainer: R Core Team R-core@ r-project.org, Description: R statistical functions, License: Part of R 3.0.2, Built: R 3.0.2; x86_64-w64-mingw32; 2013-09-25 15:39:42 UTC; windows

[21] Morgan, R.M., Davies, G., Balestri, F., Bull, P. The Recovery of Pollen Evidence from Documents and Its Forensic Implications. Science \& Justice 53.4 (2013): 375-84. 
[22] Pline. Use of Digital Image Analysis, Viability Stains, and Germination Assays to Estimate Conventional and Glyphosate-resistant Cotton Pollen Viability. Crop Science 42.6 (2002): 2193.

[23] Abbott, S. Mycotoxins and Indoor Air Molds. Indoor Environment Connections, Vol. 3, Issue 4, 2002. 1-3.

[24] Schneider, C., Rasband, W., Eliceiri, K. NIH Image to ImageJ: 25 Years of Image Analysis. Nature Methods 9.7 (2012): 671-75.

[25] Ferreira, T., Rasband, W. ImageJ User Guide. 〈http://imagej.nih.gov/ij/docs/user-guide.pdf>.

[26] Jones, G.D. Pollen Analysis for Pollination Research, Unacetolyzed Pollen. Journal of Pollination Ecology 9.13 (2012): 96-107.

[27] Gardner, R.M, Practical Crime Scene Processing and Investigation, Second Edition. Chapter 2. The Nature of Physical Evidence. (2011): 37

[28] The Centre of Forensic Sciences Public Safety Division Ministry of Community Safety and Correctional Services; Handbook of Forensic Evidence for the Investigator. (2011). 16, 31

[29] Ojena, S.M., Law and Order, New Electrostatic Process Recovers Visible and Invisible Dust Particles at Crime Scenes. July 1988.

[30] National Institute of Health; ImageJ User Guide" IJ 1.45 


\section{Appendix A: R Scripts}

ANOVA:

setwd("D:/Graduate Research/ANOVA_data")

getwd()

MR=read.table("All_Results_Means.txt",header=TRUE, sep="|t", dec=".",

strip. white=TRUE,blank.lines.skip=TRUE, na.strings="NA", fill=TRUE)

MR

MR_aov=aov(Pollen_Count_2 Recovery_Methods*Surface,data=MR)

MR_aov

summary(MR_aov)

print(model.tables(MR_aov,"means"),digits=3)

Tukey:

TukeyHSD $($ MR_aov, ordered $=$ FALSE, conf.level $=0.95)$

\section{BOXPLOTS:}

setwd("D:/Graduate Research/ANOVA_data")

getwd()

MR=read.table("Cans_Results.txt",header=TRUE, sep="|t", dec=".",

strip. white=TRUE,blank.lines.skip=TRUE, na.strings="NA", fill=TRUE)

MR

$\operatorname{par}(\mathrm{mfrow}=\mathrm{c}(1,1))$

boxplot(Pollen_Count $\sim$ Recovery_Method,data=MR,ylab="Pollen Count",main="Amount of Pollen Recovered from Metal Cans") 


\section{Appendix B: Tables of Pollen Counts}

\begin{tabular}{|c|c|c|c|c|}
\hline Surface & Recovery_Method & Sample_Number & Image_Number & Count \\
\hline Cans & TapeLift & 1 & 1 & 21 \\
\hline Cans & TapeLift & 1 & 2 & 60 \\
\hline Cans & TapeLift & 1 & 3 & 27 \\
\hline Cans & TapeLift & 1 & 4 & 31 \\
\hline Cans & TapeLift & 1 & 5 & 53 \\
\hline Cans & TapeLift & 1 & 6 & 47 \\
\hline Cans & TapeLift & 1 & 7 & 41 \\
\hline Cans & TapeLift & 1 & 8 & 45 \\
\hline Cans & TapeLift & 1 & 9 & 32 \\
\hline Cans & TapeLift & 1 & 10 & 26 \\
\hline Cans & TapeLift & 1 & 11 & 9 \\
\hline Cans & TapeLift & 1 & 12 & 23 \\
\hline Cans & TapeLift & 1 & 13 & 31 \\
\hline Cans & TapeLift & 1 & 14 & 64 \\
\hline Cans & TapeLift & 1 & 15 & 55 \\
\hline Cans & TapeLift & 1 & 16 & 15 \\
\hline Cans & TapeLift & 1 & 17 & 25 \\
\hline Cans & TapeLift & 1 & 18 & 39 \\
\hline Cans & TapeLift & 1 & 19 & 50 \\
\hline Cans & TapeLift & 1 & 20 & 6 \\
\hline Cans & TapeLift & 1 & 21 & 51 \\
\hline Cans & TapeLift & 1 & 22 & 14 \\
\hline Cans & TapeLift & 1 & 23 & 27 \\
\hline Cans & TapeLift & 1 & 24 & 50 \\
\hline Cans & TapeLift & 2 & 1 & 46 \\
\hline Cans & TapeLift & 2 & 2 & 43 \\
\hline Cans & TapeLift & 2 & 3 & 33 \\
\hline Cans & TapeLift & 2 & 4 & 35 \\
\hline Cans & TapeLift & 2 & 5 & 51 \\
\hline Cans & TapeLift & 2 & 6 & 58 \\
\hline Cans & TapeLift & 2 & 7 & 59 \\
\hline Cans & TapeLift & 2 & 8 & 28 \\
\hline Cans & TapeLift & 2 & 9 & 31 \\
\hline Cans & TapeLift & 2 & 10 & 31 \\
\hline Cans & TapeLift & 2 & 11 & 47 \\
\hline Cans & TapeLift & 2 & 12 & 23 \\
\hline Cans & TapeLift & 2 & 13 & 64 \\
\hline Cans & TapeLift & 2 & 14 & 26 \\
\hline
\end{tabular}




\begin{tabular}{|c|c|c|c|}
\hline Cans & TapeLift & 2 & 15 \\
\hline Cans & TapeLift & 2 & 16 \\
\hline Cans & TapeLift & 2 & 17 \\
\hline Cans & TapeLift & 2 & 18 \\
\hline Cans & TapeLift & 3 & 1 \\
\hline Cans & TapeLift & 3 & 2 \\
\hline Cans & TapeLift & 3 & 3 \\
\hline Cans & TapeLift & 3 & 4 \\
\hline Cans & TapeLift & 3 & 5 \\
\hline Cans & TapeLift & 3 & 6 \\
\hline Cans & TapeLift & 3 & 7 \\
\hline Cans & TapeLift & 3 & 8 \\
\hline Cans & TapeLift & 3 & 9 \\
\hline Cans & TapeLift & 3 & 10 \\
\hline Cans & TapeLift & 3 & 11 \\
\hline Cans & TapeLift & 3 & 12 \\
\hline Cans & TapeLift & 3 & 13 \\
\hline Cans & TapeLift & 3 & 14 \\
\hline Cans & TapeLift & 3 & 15 \\
\hline Cans & TapeLift & 3 & 16 \\
\hline Cans & TapeLift & 3 & 17 \\
\hline Cans & TapeLift & 3 & 18 \\
\hline Cans & TapeLift & 3 & 19 \\
\hline Cans & TapeLift & 3 & 20 \\
\hline Cans & TapeLift & 3 & 21 \\
\hline Cans & TapeLift & 3 & 22 \\
\hline Cans & TapeLift & 4 & 1 \\
\hline Cans & TapeLift & 4 & 2 \\
\hline Cans & TapeLift & 4 & 3 \\
\hline Cans & TapeLift & 4 & 4 \\
\hline Cans & TapeLift & 4 & 5 \\
\hline Cans & TapeLift & 4 & 6 \\
\hline Cans & TapeLift & 4 & 7 \\
\hline Cans & TapeLift & 4 & 8 \\
\hline Cans & TapeLift & 4 & 9 \\
\hline Cans & TapeLift & 4 & 10 \\
\hline Cans & TapeLift & 4 & 11 \\
\hline Cans & TapeLift & 4 & 12 \\
\hline Cans & TapeLift & 4 & 13 \\
\hline Cans & TapeLift & 4 & 14 \\
\hline Cans & TapeLift & 4 & 15 \\
\hline Cans & TapeLift & 4 & 16 \\
\hline Cans & TapeLift & 4 & 17 \\
\hline Cans & TapeLift & 4 & 18 \\
\hline Cans & TapeLift & 4 & 19 \\
\hline
\end{tabular}




\begin{tabular}{|c|c|c|c|}
\hline Cans & TapeLift & 4 & 20 \\
\hline Cans & TapeLift & 4 & 21 \\
\hline Cans & TapeLift & 4 & 22 \\
\hline Cans & TapeLift & 4 & 23 \\
\hline Cans & TapeLift & 4 & 24 \\
\hline Cans & TapeLift & 4 & 25 \\
\hline Cans & TapeLift & 4 & 26 \\
\hline Cans & TapeLift & 4 & 27 \\
\hline Cans & TapeLift & 4 & 28 \\
\hline Cans & TapeLift & 4 & 29 \\
\hline Cans & TapeLift & 4 & 30 \\
\hline Cans & TapeLift & 4 & 31 \\
\hline Cans & TapeLift & 4 & 32 \\
\hline Cans & TapeLift & 4 & 33 \\
\hline Cans & TapeLift & 4 & 34 \\
\hline Cans & TapeLift & 5 & 1 \\
\hline Cans & TapeLift & 5 & 2 \\
\hline Cans & TapeLift & 5 & 3 \\
\hline Cans & TapeLift & 5 & 4 \\
\hline Cans & TapeLift & 5 & 5 \\
\hline Cans & TapeLift & 5 & 6 \\
\hline Cans & TapeLift & 5 & 7 \\
\hline Cans & TapeLift & 5 & 8 \\
\hline Cans & TapeLift & 5 & 9 \\
\hline Cans & TapeLift & 5 & 10 \\
\hline Cans & TapeLift & 5 & 11 \\
\hline Cans & TapeLift & 5 & 12 \\
\hline Cans & TapeLift & 5 & 13 \\
\hline Cans & TapeLift & 5 & 14 \\
\hline Cans & TapeLift & 5 & 15 \\
\hline Cans & TapeLift & 5 & 16 \\
\hline Cans & TapeLift & 5 & 17 \\
\hline Cans & TapeLift & 5 & 18 \\
\hline Cans & TapeLift & 5 & 19 \\
\hline Cans & TapeLift & 5 & 20 \\
\hline Cans & TapeLift & 5 & 21 \\
\hline Cans & TapeLift & 5 & 22 \\
\hline Cans & TapeLift & 5 & 23 \\
\hline Cans & TapeLift & 5 & 24 \\
\hline Cans & TapeLift & 5 & 25 \\
\hline Cans & TapeLift & 5 & 26 \\
\hline Cans & TapeLift & 5 & 27 \\
\hline Cans & TapeLift & 5 & 28 \\
\hline Cans & TapeLift & 5 & 29 \\
\hline Cans & TapeLift & 5 & 30 \\
\hline
\end{tabular}




\begin{tabular}{|c|c|c|c|c|}
\hline Cans & TapeLift & 5 & 31 & 60 \\
\hline Cans & TapeLift & 5 & 32 & 47 \\
\hline Cans & TapeLift & 5 & 33 & 26 \\
\hline Cans & TapeLift & 5 & 34 & 34 \\
\hline Cans & TapeLift & 5 & 35 & 44 \\
\hline Cans & TapeLift & 5 & 36 & 51 \\
\hline Cans & TapeLift & 6 & 1 & 38 \\
\hline Cans & TapeLift & 6 & 2 & 46 \\
\hline Cans & TapeLift & 6 & 3 & 35 \\
\hline Cans & TapeLift & 6 & 4 & 43 \\
\hline Cans & TapeLift & 6 & 5 & 50 \\
\hline Cans & TapeLift & 6 & 6 & 52 \\
\hline Cans & TapeLift & 6 & 7 & 38 \\
\hline Cans & TapeLift & 6 & 8 & 36 \\
\hline Cans & TapeLift & 6 & 9 & 42 \\
\hline Cans & TapeLift & 6 & 10 & 22 \\
\hline Cans & TapeLift & 6 & 11 & 50 \\
\hline Cans & TapeLift & 6 & 12 & 44 \\
\hline Cans & TapeLift & 6 & 13 & 55 \\
\hline Cans & TapeLift & 6 & 14 & 59 \\
\hline Cans & TapeLift & 6 & 15 & 47 \\
\hline Cans & TapeLift & 6 & 16 & 36 \\
\hline Cans & TapeLift & 6 & 17 & 43 \\
\hline Cans & TapeLift & 6 & 18 & 30 \\
\hline Cans & TapeLift & 6 & 19 & 56 \\
\hline Cans & TapeLift & 6 & 20 & 35 \\
\hline Cans & TapeLift & 6 & 21 & 41 \\
\hline Cans & TapeLift & 6 & 22 & 41 \\
\hline Cans & TapeLift & 6 & 23 & 28 \\
\hline Cans & TapeLift & 6 & 24 & 47 \\
\hline Cans & TapeLift & 6 & 25 & 49 \\
\hline Cans & TapeLift & 6 & 26 & 41 \\
\hline Cans & TapeLift & 6 & 27 & 30 \\
\hline Cans & TapeLift & 6 & 28 & 65 \\
\hline Cans & TapeLift & 6 & 29 & 45 \\
\hline Cans & TapeLift & 6 & 30 & 36 \\
\hline Cans & TapeLift & 6 & 31 & 35 \\
\hline Cans & TapeLift & 6 & 32 & 54 \\
\hline Cans & TapeLift & 6 & 33 & 37 \\
\hline Cans & TapeLift & 6 & 34 & 26 \\
\hline Cans & TapeLift & 6 & 35 & 28 \\
\hline Cans & TapeLift & 7 & 1 & 41 \\
\hline Cans & TapeLift & 7 & 2 & 36 \\
\hline Cans & TapeLift & 7 & 3 & 51 \\
\hline Cans & TapeLift & 7 & 4 & 31 \\
\hline
\end{tabular}




\begin{tabular}{|c|c|c|c|}
\hline Cans & TapeLift & 7 & 5 \\
\hline Cans & TapeLift & 7 & 6 \\
\hline Cans & TapeLift & 7 & 7 \\
\hline Cans & TapeLift & 7 & 8 \\
\hline Cans & TapeLift & 7 & 9 \\
\hline Cans & TapeLift & 7 & 10 \\
\hline Cans & TapeLift & 7 & 11 \\
\hline Cans & TapeLift & 7 & 12 \\
\hline Cans & TapeLift & 7 & 13 \\
\hline Cans & TapeLift & 7 & 14 \\
\hline Cans & TapeLift & 7 & 15 \\
\hline Cans & TapeLift & 7 & 16 \\
\hline Cans & TapeLift & 7 & 17 \\
\hline Cans & TapeLift & 7 & 18 \\
\hline Cans & TapeLift & 7 & 19 \\
\hline Cans & TapeLift & 7 & 20 \\
\hline Cans & TapeLift & 7 & 21 \\
\hline Cans & TapeLift & 7 & 22 \\
\hline Cans & TapeLift & 7 & 23 \\
\hline Cans & TapeLift & 7 & 24 \\
\hline Cans & TapeLift & 7 & 25 \\
\hline Cans & TapeLift & 7 & 26 \\
\hline Cans & TapeLift & 7 & 27 \\
\hline Cans & TapeLift & 7 & 28 \\
\hline Cans & TapeLift & 7 & 29 \\
\hline Cans & TapeLift & 7 & 30 \\
\hline Cans & TapeLift & 7 & 31 \\
\hline Cans & TapeLift & 7 & 32 \\
\hline Cans & TapeLift & 7 & 33 \\
\hline Cans & TapeLift & 8 & 1 \\
\hline Cans & TapeLift & 8 & 2 \\
\hline Cans & TapeLift & 8 & 3 \\
\hline Cans & TapeLift & 8 & 4 \\
\hline Cans & TapeLift & 8 & 5 \\
\hline Cans & TapeLift & 8 & 6 \\
\hline Cans & TapeLift & 8 & 7 \\
\hline Cans & TapeLift & 8 & 8 \\
\hline Cans & TapeLift & 8 & 9 \\
\hline Cans & TapeLift & 8 & 10 \\
\hline Cans & TapeLift & 8 & 11 \\
\hline Cans & TapeLift & 8 & 12 \\
\hline Cans & TapeLift & 8 & 13 \\
\hline Cans & TapeLift & 8 & 14 \\
\hline Cans & TapeLift & 8 & 15 \\
\hline Cans & TapeLift & 8 & 16 \\
\hline
\end{tabular}




\begin{tabular}{|c|c|c|c|c|}
\hline Cans & TapeLift & 8 & 17 & 69 \\
\hline Cans & TapeLift & 8 & 18 & 22 \\
\hline Cans & TapeLift & 8 & 19 & 45 \\
\hline Cans & TapeLift & 8 & 20 & 30 \\
\hline Cans & TapeLift & 8 & 21 & 36 \\
\hline Cans & TapeLift & 8 & 22 & 39 \\
\hline Cans & TapeLift & 8 & 23 & 49 \\
\hline Cans & TapeLift & 8 & 24 & 55 \\
\hline Cans & TapeLift & 8 & 25 & 58 \\
\hline Cans & TapeLift & 8 & 26 & 61 \\
\hline Cans & TapeLift & 8 & 27 & 47 \\
\hline Cans & TapeLift & 8 & 28 & 66 \\
\hline Cans & TapeLift & 8 & 29 & 22 \\
\hline Cans & TapeLift & 8 & 30 & 49 \\
\hline Cans & TapeLift & 8 & 31 & 28 \\
\hline Cans & TapeLift & 8 & 32 & 20 \\
\hline Cans & TapeLift & 8 & 33 & 59 \\
\hline Cans & TapeLift & 8 & 34 & 45 \\
\hline Cans & TapeLift & 8 & 35 & 30 \\
\hline Cans & TapeLift & 9 & 1 & 33 \\
\hline Cans & TapeLift & 9 & 2 & 40 \\
\hline Cans & TapeLift & 9 & 3 & 62 \\
\hline Cans & TapeLift & 9 & 4 & 33 \\
\hline Cans & TapeLift & 9 & 5 & 49 \\
\hline Cans & TapeLift & 9 & 6 & 35 \\
\hline Cans & TapeLift & 9 & 7 & 29 \\
\hline Cans & TapeLift & 9 & 8 & 53 \\
\hline Cans & TapeLift & 9 & 9 & 55 \\
\hline Cans & TapeLift & 9 & 10 & 48 \\
\hline Cans & TapeLift & 9 & 11 & 50 \\
\hline Cans & TapeLift & 9 & 12 & 43 \\
\hline Cans & TapeLift & 9 & 13 & 42 \\
\hline Cans & TapeLift & 9 & 14 & 55 \\
\hline Cans & TapeLift & 9 & 15 & 35 \\
\hline Cans & TapeLift & 9 & 16 & 29 \\
\hline Cans & TapeLift & 9 & 17 & 47 \\
\hline Cans & TapeLift & 9 & 18 & 31 \\
\hline Cans & TapeLift & 9 & 19 & 39 \\
\hline Cans & TapeLift & 9 & 20 & 60 \\
\hline Cans & TapeLift & 9 & 21 & 66 \\
\hline Cans & TapeLift & 9 & 22 & 66 \\
\hline Cans & TapeLift & 9 & 23 & 61 \\
\hline Cans & TapeLift & 9 & 24 & 63 \\
\hline Cans & TapeLift & 9 & 25 & 52 \\
\hline Cans & TapeLift & 9 & 26 & 55 \\
\hline
\end{tabular}




\begin{tabular}{|c|c|c|c|c|}
\hline Cans & TapeLift & 9 & 27 & 35 \\
\hline Cans & TapeLift & 10 & 1 & 37 \\
\hline Cans & TapeLift & 10 & 2 & 52 \\
\hline Cans & TapeLift & 10 & 3 & 34 \\
\hline Cans & TapeLift & 10 & 4 & 50 \\
\hline Cans & TapeLift & 10 & 5 & 58 \\
\hline Cans & TapeLift & 10 & 6 & 39 \\
\hline Cans & TapeLift & 10 & 7 & 49 \\
\hline Cans & TapeLift & 10 & 8 & 52 \\
\hline Cans & TapeLift & 10 & 9 & 51 \\
\hline Cans & TapeLift & 10 & 10 & 56 \\
\hline Cans & TapeLift & 10 & 11 & 25 \\
\hline Cans & TapeLift & 10 & 12 & 4 \\
\hline Cans & TapeLift & 10 & 13 & 31 \\
\hline Cans & TapeLift & 10 & 14 & 48 \\
\hline Cans & TapeLift & 10 & 15 & 17 \\
\hline Cans & TapeLift & 10 & 16 & 34 \\
\hline Cans & TapeLift & 10 & 17 & 54 \\
\hline Cans & TapeLift & 10 & 18 & 46 \\
\hline Cans & TapeLift & 10 & 19 & 53 \\
\hline Cans & TapeLift & 10 & 20 & 51 \\
\hline Cans & TapeLift & 10 & 21 & 51 \\
\hline Cans & TapeLift & 10 & 22 & 46 \\
\hline Cans & TapeLift & 10 & 23 & 44 \\
\hline Cans & TapeLift & 10 & 24 & 47 \\
\hline Cans & TapeLift & 10 & 25 & 90 \\
\hline Cans & TapeLift & 11 & 1 & 28 \\
\hline Cans & TapeLift & 11 & 2 & 71 \\
\hline Cans & TapeLift & 11 & 3 & 52 \\
\hline Cans & TapeLift & 11 & 4 & 59 \\
\hline Cans & TapeLift & 11 & 5 & 53 \\
\hline Cans & TapeLift & 11 & 6 & 74 \\
\hline Cans & TapeLift & 11 & 7 & 66 \\
\hline Cans & TapeLift & 11 & 8 & 49 \\
\hline Cans & TapeLift & 11 & 9 & 68 \\
\hline Cans & TapeLift & 11 & 10 & 59 \\
\hline Cans & TapeLift & 11 & 11 & 79 \\
\hline Cans & TapeLift & 11 & 12 & 38 \\
\hline Cans & TapeLift & 11 & 13 & 38 \\
\hline Cans & TapeLift & 11 & 14 & 55 \\
\hline Cans & TapeLift & 11 & 15 & 51 \\
\hline Cans & TapeLift & 11 & 16 & 53 \\
\hline Cans & TapeLift & 11 & 17 & 40 \\
\hline Cans & TapeLift & 11 & 18 & 48 \\
\hline Cans & TapeLift & 11 & 19 & 34 \\
\hline
\end{tabular}




\begin{tabular}{|c|c|c|c|c|}
\hline Cans & TapeLift & 11 & 20 & 72 \\
\hline Cans & TapeLift & 11 & 21 & 48 \\
\hline Cans & TapeLift & 11 & 22 & 51 \\
\hline Cans & TapeLift & 11 & 23 & 29 \\
\hline Cans & TapeLift & 11 & 24 & 22 \\
\hline Cans & TapeLift & 11 & 25 & 22 \\
\hline Cans & TapeLift & 11 & 26 & 49 \\
\hline Cans & TapeLift & 11 & 27 & 56 \\
\hline Cans & TapeLift & 11 & 28 & 43 \\
\hline Cans & TapeLift & 11 & 29 & 31 \\
\hline Cans & TapeLift & 11 & 30 & 46 \\
\hline Cans & TapeLift & 11 & 31 & 64 \\
\hline Cans & TapeLift & 11 & 32 & 40 \\
\hline Cans & TapeLift & 11 & 33 & 32 \\
\hline Cans & TapeLift & 11 & 34 & 28 \\
\hline Cans & TapeLift & 11 & 35 & 47 \\
\hline Cans & TapeLift & 11 & 36 & 65 \\
\hline Cans & TapeLift & 11 & 37 & 60 \\
\hline Cans & TapeLift & 11 & 38 & 48 \\
\hline Cans & TapeLift & 11 & 39 & 45 \\
\hline Cans & TapeLift & 11 & 40 & 45 \\
\hline Cans & TapeLift & 12 & 1 & 58 \\
\hline Cans & TapeLift & 12 & 2 & 83 \\
\hline Cans & TapeLift & 12 & 3 & 82 \\
\hline Cans & TapeLift & 12 & 4 & 57 \\
\hline Cans & TapeLift & 12 & 5 & 113 \\
\hline Cans & TapeLift & 12 & 6 & 35 \\
\hline Cans & TapeLift & 12 & 7 & 57 \\
\hline Cans & TapeLift & 12 & 8 & 37 \\
\hline Cans & TapeLift & 12 & 9 & 105 \\
\hline Cans & TapeLift & 12 & 10 & 52 \\
\hline Cans & TapeLift & 12 & 11 & 25 \\
\hline Cans & TapeLift & 12 & 12 & 58 \\
\hline Cans & TapeLift & 12 & 13 & 60 \\
\hline Cans & TapeLift & 12 & 14 & 52 \\
\hline Cans & TapeLift & 12 & 15 & 130 \\
\hline Cans & TapeLift & 12 & 16 & 58 \\
\hline Cans & TapeLift & 12 & 17 & 41 \\
\hline Cans & TapeLift & 12 & 18 & 62 \\
\hline Cans & TapeLift & 12 & 19 & 51 \\
\hline Cans & TapeLift & 12 & 20 & 44 \\
\hline Cans & TapeLift & 12 & 21 & 80 \\
\hline Cans & TapeLift & 12 & 22 & 52 \\
\hline Cans & TapeLift & 12 & 23 & 63 \\
\hline Cans & TapeLift & 12 & 24 & 36 \\
\hline
\end{tabular}




\begin{tabular}{|c|c|c|c|c|}
\hline Cans & TapeLift & 12 & 25 & 62 \\
\hline Cans & TapeLift & 12 & 26 & 39 \\
\hline Cans & TapeLift & 12 & 27 & 65 \\
\hline Cans & TapeLift & 12 & 28 & 51 \\
\hline Cans & TapeLift & 12 & 29 & 56 \\
\hline Cans & TapeLift & 12 & 30 & 56 \\
\hline Cans & TapeLift & 12 & 31 & 41 \\
\hline Cans & TapeLift & 13 & 1 & 51 \\
\hline Cans & TapeLift & 13 & 2 & 53 \\
\hline Cans & TapeLift & 13 & 3 & 33 \\
\hline Cans & TapeLift & 13 & 4 & 59 \\
\hline Cans & TapeLift & 13 & 5 & 49 \\
\hline Cans & TapeLift & 13 & 6 & 48 \\
\hline Cans & TapeLift & 13 & 7 & 59 \\
\hline Cans & TapeLift & 13 & 8 & 58 \\
\hline Cans & TapeLift & 13 & 9 & 68 \\
\hline Cans & TapeLift & 13 & 10 & 56 \\
\hline Cans & TapeLift & 13 & 11 & 60 \\
\hline Cans & TapeLift & 13 & 12 & 38 \\
\hline Cans & TapeLift & 13 & 13 & 46 \\
\hline Cans & TapeLift & 13 & 14 & 55 \\
\hline Cans & TapeLift & 13 & 15 & 43 \\
\hline Cans & TapeLift & 13 & 16 & 43 \\
\hline Cans & TapeLift & 13 & 17 & 40 \\
\hline Cans & TapeLift & 13 & 18 & 61 \\
\hline Cans & TapeLift & 13 & 19 & 51 \\
\hline Cans & TapeLift & 13 & 20 & 58 \\
\hline Cans & TapeLift & 13 & 21 & 68 \\
\hline Cans & TapeLift & 13 & 22 & 50 \\
\hline Cans & TapeLift & 13 & 23 & 23 \\
\hline Cans & TapeLift & 13 & 24 & 28 \\
\hline Cans & TapeLift & 13 & 25 & 21 \\
\hline Cans & TapeLift & 13 & 26 & 53 \\
\hline Cans & TapeLift & 13 & 27 & 74 \\
\hline Cans & TapeLift & 13 & 28 & 41 \\
\hline Cans & TapeLift & 13 & 29 & 25 \\
\hline Cans & TapeLift & 13 & 30 & 18 \\
\hline Cans & TapeLift & 13 & 31 & 9 \\
\hline Cans & TapeLift & 13 & 32 & 33 \\
\hline Cans & TapeLift & 13 & 33 & 46 \\
\hline Cans & TapeLift & 13 & 34 & 25 \\
\hline Cans & TapeLift & 13 & 35 & 40 \\
\hline Cans & TapeLift & 13 & 36 & 54 \\
\hline Cans & TapeLift & 13 & 37 & 40 \\
\hline Cans & TapeLift & 13 & 38 & 31 \\
\hline
\end{tabular}




\begin{tabular}{|c|c|c|c|c|}
\hline Cans & TapeLift & 13 & 39 & 27 \\
\hline Cans & TapeLift & 13 & 40 & 76 \\
\hline Cans & TapeLift & 13 & 41 & 55 \\
\hline Cans & TapeLift & 13 & 42 & 71 \\
\hline Cans & TapeLift & 14 & 1 & 32 \\
\hline Cans & TapeLift & 14 & 2 & 29 \\
\hline Cans & TapeLift & 14 & 3 & 63 \\
\hline Cans & TapeLift & 14 & 4 & 30 \\
\hline Cans & TapeLift & 14 & 5 & 43 \\
\hline Cans & TapeLift & 14 & 6 & 23 \\
\hline Cans & TapeLift & 14 & 7 & 29 \\
\hline Cans & TapeLift & 14 & 8 & 41 \\
\hline Cans & TapeLift & 14 & 9 & 28 \\
\hline Cans & TapeLift & 14 & 10 & 50 \\
\hline Cans & TapeLift & 14 & 11 & 47 \\
\hline Cans & TapeLift & 14 & 12 & 38 \\
\hline Cans & TapeLift & 14 & 13 & 39 \\
\hline Cans & TapeLift & 14 & 14 & 48 \\
\hline Cans & TapeLift & 14 & 15 & 52 \\
\hline Cans & TapeLift & 14 & 16 & 34 \\
\hline Cans & TapeLift & 14 & 17 & 34 \\
\hline Cans & TapeLift & 14 & 18 & 52 \\
\hline Cans & TapeLift & 14 & 19 & 70 \\
\hline Cans & TapeLift & 14 & 20 & 54 \\
\hline Cans & TapeLift & 14 & 21 & 32 \\
\hline Cans & TapeLift & 14 & 22 & 59 \\
\hline Cans & TapeLift & 14 & 23 & 52 \\
\hline Cans & TapeLift & 14 & 24 & 57 \\
\hline Cans & TapeLift & 14 & 25 & 28 \\
\hline Cans & TapeLift & 14 & 26 & 39 \\
\hline Cans & TapeLift & 14 & 27 & 50 \\
\hline Cans & TapeLift & 14 & 28 & 47 \\
\hline Cans & TapeLift & 14 & 29 & 45 \\
\hline Cans & TapeLift & 14 & 30 & 28 \\
\hline Cans & TapeLift & 14 & 31 & 57 \\
\hline Cans & TapeLift & 14 & 32 & 48 \\
\hline Cans & TapeLift & 14 & 33 & 35 \\
\hline Cans & TapeLift & 14 & 34 & 50 \\
\hline Cans & TapeLift & 15 & 1 & 30 \\
\hline Cans & TapeLift & 15 & 2 & 27 \\
\hline Cans & TapeLift & 15 & 3 & 32 \\
\hline Cans & TapeLift & 15 & 4 & 48 \\
\hline Cans & TapeLift & 15 & 5 & 26 \\
\hline Cans & TapeLift & 15 & 6 & 73 \\
\hline Cans & TapeLift & 15 & 7 & 71 \\
\hline
\end{tabular}




\begin{tabular}{|c|c|c|c|c|}
\hline Cans & TapeLift & 15 & 8 & 51 \\
\hline Cans & TapeLift & 15 & 9 & 40 \\
\hline Cans & TapeLift & 15 & 10 & 54 \\
\hline Cans & TapeLift & 15 & 11 & 39 \\
\hline Cans & TapeLift & 15 & 12 & 28 \\
\hline Cans & TapeLift & 15 & 13 & 42 \\
\hline Cans & TapeLift & 15 & 14 & 37 \\
\hline Cans & TapeLift & 15 & 15 & 28 \\
\hline Cans & TapeLift & 15 & 16 & 36 \\
\hline Cans & TapeLift & 15 & 17 & 43 \\
\hline Cans & TapeLift & 15 & 18 & 48 \\
\hline Cans & TapeLift & 15 & 19 & 41 \\
\hline Cans & TapeLift & 15 & 20 & 81 \\
\hline Cans & TapeLift & 15 & 21 & 43 \\
\hline Cans & TapeLift & 15 & 22 & 50 \\
\hline Cans & TapeLift & 15 & 23 & 53 \\
\hline Cans & TapeLift & 15 & 24 & 41 \\
\hline Cans & TapeLift & 15 & 25 & 41 \\
\hline Cans & TapeLift & 15 & 26 & 49 \\
\hline Cans & TapeLift & 15 & 27 & 43 \\
\hline Cans & TapeLift & 15 & 28 & 55 \\
\hline Cans & TapeLift & 15 & 29 & 65 \\
\hline Cans & TapeLift & 15 & 30 & 34 \\
\hline Cans & TapeLift & 15 & 31 & 41 \\
\hline Cans & TapeLift & 15 & 32 & 49 \\
\hline Cans & TapeLift & 15 & 33 & 40 \\
\hline Cans & TapeLift & 15 & 34 & 66 \\
\hline Cans & TapeLift & 15 & 35 & 34 \\
\hline Cans & TapeLift & 15 & 36 & 56 \\
\hline Cans & TapeLift & 15 & 37 & 56 \\
\hline Cans & TapeLift & 15 & 38 & 55 \\
\hline Cans & TapeLift & 15 & 39 & 67 \\
\hline Cans & TapeLift & 15 & 40 & 38 \\
\hline Cans & TapeLift & 15 & 41 & 43 \\
\hline Cans & TapeLift & 15 & 42 & 34 \\
\hline Cans & TapeLift & 15 & 43 & 58 \\
\hline Cans & TapeLift & 15 & 44 & 42 \\
\hline Cans & TapeLift & 15 & 45 & 63 \\
\hline Cans & TapeLift & 16 & 1 & 50 \\
\hline Cans & TapeLift & 16 & 2 & 44 \\
\hline Cans & TapeLift & 16 & 3 & 32 \\
\hline Cans & TapeLift & 16 & 4 & 41 \\
\hline Cans & TapeLift & 16 & 5 & 43 \\
\hline Cans & TapeLift & 16 & 6 & 40 \\
\hline Cans & TapeLift & 16 & 7 & 64 \\
\hline
\end{tabular}




\begin{tabular}{|c|c|c|c|}
\hline Cans & TapeLift & 16 & 8 \\
\hline Cans & TapeLift & 16 & 9 \\
\hline Cans & TapeLift & 16 & 10 \\
\hline Cans & TapeLift & 16 & 11 \\
\hline Cans & TapeLift & 16 & 12 \\
\hline Cans & TapeLift & 16 & 13 \\
\hline Cans & TapeLift & 16 & 14 \\
\hline Cans & TapeLift & 16 & 15 \\
\hline Cans & TapeLift & 16 & 16 \\
\hline Cans & TapeLift & 16 & 17 \\
\hline Cans & TapeLift & 16 & 18 \\
\hline Cans & TapeLift & 16 & 19 \\
\hline Cans & TapeLift & 16 & 20 \\
\hline Cans & TapeLift & 16 & 21 \\
\hline Cans & TapeLift & 16 & 22 \\
\hline Cans & TapeLift & 16 & 23 \\
\hline Cans & TapeLift & 16 & 24 \\
\hline Cans & TapeLift & 16 & 25 \\
\hline Cans & TapeLift & 16 & 26 \\
\hline Cans & TapeLift & 16 & 27 \\
\hline Cans & TapeLift & 16 & 28 \\
\hline Cans & TapeLift & 16 & 29 \\
\hline Cans & TapeLift & 16 & 30 \\
\hline Cans & TapeLift & 16 & 31 \\
\hline Cans & TapeLift & 16 & 32 \\
\hline Cans & TapeLift & 16 & 33 \\
\hline Cans & TapeLift & 16 & 34 \\
\hline Cans & TapeLift & 16 & 35 \\
\hline Cans & TapeLift & 16 & 36 \\
\hline Cans & TapeLift & 17 & 1 \\
\hline Cans & TapeLift & 17 & 2 \\
\hline Cans & TapeLift & 17 & 3 \\
\hline Cans & TapeLift & 17 & 4 \\
\hline Cans & TapeLift & 17 & 5 \\
\hline Cans & TapeLift & 17 & 6 \\
\hline Cans & TapeLift & 17 & 7 \\
\hline Cans & TapeLift & 17 & 8 \\
\hline Cans & TapeLift & 17 & 9 \\
\hline Cans & TapeLift & 17 & 10 \\
\hline Cans & TapeLift & 17 & 11 \\
\hline Cans & TapeLift & 17 & 12 \\
\hline Cans & TapeLift & 17 & 13 \\
\hline Cans & TapeLift & 17 & 14 \\
\hline Cans & TapeLift & 17 & 15 \\
\hline Cans & TapeLift & 17 & 16 \\
\hline
\end{tabular}




\begin{tabular}{|c|c|c|c|c|}
\hline Cans & TapeLift & 17 & 17 & 35 \\
\hline Cans & TapeLift & 17 & 18 & 42 \\
\hline Cans & TapeLift & 17 & 19 & 33 \\
\hline Cans & TapeLift & 17 & 20 & 30 \\
\hline Cans & TapeLift & 17 & 21 & 31 \\
\hline Cans & TapeLift & 17 & 22 & 31 \\
\hline Cans & TapeLift & 17 & 23 & 50 \\
\hline Cans & TapeLift & 17 & 24 & 34 \\
\hline Cans & TapeLift & 17 & 25 & 44 \\
\hline Cans & TapeLift & 17 & 26 & 93 \\
\hline Cans & TapeLift & 17 & 27 & 54 \\
\hline Cans & TapeLift & 17 & 28 & 66 \\
\hline Cans & TapeLift & 17 & 29 & 83 \\
\hline Cans & TapeLift & 17 & 30 & 68 \\
\hline Cans & TapeLift & 17 & 31 & 73 \\
\hline Cans & TapeLift & 17 & 32 & 63 \\
\hline Cans & TapeLift & 17 & 33 & 50 \\
\hline Cans & TapeLift & 17 & 34 & 44 \\
\hline Cans & TapeLift & 17 & 35 & 55 \\
\hline Cans & TapeLift & 17 & 36 & 69 \\
\hline Cans & TapeLift & 17 & 37 & 53 \\
\hline Cans & TapeLift & 17 & 38 & 47 \\
\hline Cans & TapeLift & 17 & 39 & 59 \\
\hline Cans & TapeLift & 17 & 40 & 65 \\
\hline Cans & TapeLift & 18 & 1 & 42 \\
\hline Cans & TapeLift & 18 & 2 & 38 \\
\hline Cans & TapeLift & 18 & 3 & 32 \\
\hline Cans & TapeLift & 18 & 4 & 47 \\
\hline Cans & TapeLift & 18 & 5 & 52 \\
\hline Cans & TapeLift & 18 & 6 & 41 \\
\hline Cans & TapeLift & 18 & 7 & 64 \\
\hline Cans & TapeLift & 18 & 8 & 55 \\
\hline Cans & TapeLift & 18 & 9 & 49 \\
\hline Cans & TapeLift & 18 & 10 & 58 \\
\hline Cans & TapeLift & 18 & 11 & 53 \\
\hline Cans & TapeLift & 18 & 12 & 32 \\
\hline Cans & TapeLift & 18 & 13 & 17 \\
\hline Cans & TapeLift & 18 & 14 & 66 \\
\hline Cans & TapeLift & 18 & 15 & 43 \\
\hline Cans & TapeLift & 18 & 16 & 38 \\
\hline Cans & TapeLift & 18 & 17 & 47 \\
\hline Cans & TapeLift & 18 & 18 & 47 \\
\hline Cans & TapeLift & 18 & 19 & 49 \\
\hline Cans & TapeLift & 18 & 20 & 19 \\
\hline Cans & TapeLift & 18 & 21 & 14 \\
\hline
\end{tabular}




\begin{tabular}{|c|c|c|c|c|}
\hline Cans & TapeLift & 18 & 22 & 58 \\
\hline Cans & TapeLift & 18 & 23 & 36 \\
\hline Cans & TapeLift & 18 & 24 & 56 \\
\hline Cans & TapeLift & 18 & 25 & 63 \\
\hline Cans & TapeLift & 18 & 26 & 50 \\
\hline Cans & TapeLift & 18 & 27 & 50 \\
\hline Cans & TapeLift & 18 & 28 & 55 \\
\hline Cans & TapeLift & 18 & 29 & 47 \\
\hline Cans & TapeLift & 18 & 30 & 72 \\
\hline Cans & TapeLift & 18 & 31 & 53 \\
\hline Cans & TapeLift & 18 & 32 & 62 \\
\hline Cans & TapeLift & 18 & 33 & 49 \\
\hline Cans & TapeLift & 18 & 34 & 48 \\
\hline Cans & TapeLift & 18 & 35 & 64 \\
\hline Cans & TapeLift & 18 & 36 & 32 \\
\hline Cans & TapeLift & 18 & 37 & 69 \\
\hline Cans & TapeLift & 19 & 1 & 41 \\
\hline Cans & TapeLift & 19 & 2 & 65 \\
\hline Cans & TapeLift & 19 & 3 & 68 \\
\hline Cans & TapeLift & 19 & 4 & 34 \\
\hline Cans & TapeLift & 19 & 5 & 41 \\
\hline Cans & TapeLift & 19 & 6 & 32 \\
\hline Cans & TapeLift & 19 & 7 & 46 \\
\hline Cans & TapeLift & 19 & 8 & 39 \\
\hline Cans & TapeLift & 19 & 9 & 68 \\
\hline Cans & TapeLift & 19 & 10 & 57 \\
\hline Cans & TapeLift & 19 & 11 & 50 \\
\hline Cans & TapeLift & 19 & 12 & 38 \\
\hline Cans & TapeLift & 19 & 13 & 43 \\
\hline Cans & TapeLift & 19 & 14 & 56 \\
\hline Cans & TapeLift & 19 & 15 & 31 \\
\hline Cans & TapeLift & 19 & 16 & 56 \\
\hline Cans & TapeLift & 19 & 17 & 50 \\
\hline Cans & TapeLift & 19 & 18 & 42 \\
\hline Cans & TapeLift & 19 & 19 & 55 \\
\hline Cans & TapeLift & 19 & 20 & 36 \\
\hline Cans & TapeLift & 19 & 21 & 40 \\
\hline Cans & TapeLift & 19 & 22 & 58 \\
\hline Cans & TapeLift & 19 & 23 & 44 \\
\hline Cans & TapeLift & 19 & 24 & 53 \\
\hline Cans & TapeLift & 19 & 25 & 66 \\
\hline Cans & TapeLift & 19 & 26 & 43 \\
\hline Cans & TapeLift & 20 & 1 & 61 \\
\hline Cans & TapeLift & 20 & 2 & 34 \\
\hline Cans & TapeLift & 20 & 3 & 40 \\
\hline
\end{tabular}




\begin{tabular}{|c|c|c|c|c|}
\hline Cans & TapeLift & 20 & 4 & $4 \varepsilon$ \\
\hline Cans & TapeLift & 20 & 5 & 35 \\
\hline Cans & TapeLift & 20 & 6 & 54 \\
\hline Cans & TapeLift & 20 & 7 & 56 \\
\hline Cans & TapeLift & 20 & 8 & 38 \\
\hline Cans & TapeLift & 20 & 9 & 47 \\
\hline Cans & TapeLift & 20 & 10 & 36 \\
\hline Cans & TapeLift & 20 & 11 & 51 \\
\hline Cans & TapeLift & 20 & 12 & 36 \\
\hline Cans & TapeLift & 20 & 13 & 30 \\
\hline Cans & TapeLift & 20 & 14 & 29 \\
\hline Cans & TapeLift & 20 & 15 & 45 \\
\hline Cans & TapeLift & 20 & 16 & 54 \\
\hline Cans & TapeLift & 20 & 17 & 44 \\
\hline Cans & TapeLift & 20 & 18 & 50 \\
\hline Cans & TapeLift & 20 & 19 & 38 \\
\hline Cans & TapeLift & 20 & 20 & 38 \\
\hline Cans & TapeLift & 20 & 21 & 38 \\
\hline Cans & TapeLift & 20 & 22 & 43 \\
\hline Cans & TapeLift & 20 & 23 & 44 \\
\hline Cans & TapeLift & 20 & 24 & 24 \\
\hline Cans & TapeLift & 20 & 25 & 36 \\
\hline Cans & TapeLift & 20 & 26 & 18 \\
\hline Cans & TapeLift & 20 & 27 & 33 \\
\hline Cans & TapeLift & 20 & 28 & 86 \\
\hline Cans & TapeLift & 20 & 29 & 35 \\
\hline Cans & TapeLift & 20 & 30 & $5 \varepsilon$ \\
\hline Cans & TapeLift & 20 & 31 & 10 \\
\hline Cans & TapeLift & 20 & 32 & 30 \\
\hline Cans & TapeLift & 20 & 33 & 30 \\
\hline Cans & TapeLift & 20 & 34 & 37 \\
\hline Cans & TapeLift & 20 & 35 & 40 \\
\hline Cans & TapeLift & 20 & 36 & 46 \\
\hline Cans & TapeLift & 20 & 37 & 33 \\
\hline Cans & TapeLift & 21 & 1 & 25 \\
\hline Cans & TapeLift & 21 & 2 & 49 \\
\hline Cans & TapeLift & 21 & 3 & 39 \\
\hline Cans & TapeLift & 21 & 4 & 54 \\
\hline Cans & TapeLift & 21 & 5 & 44 \\
\hline Cans & TapeLift & 21 & 6 & 50 \\
\hline Cans & TapeLift & 21 & 7 & 31 \\
\hline Cans & TapeLift & 21 & 8 & 25 \\
\hline Cans & TapeLift & 21 & 9 & 34 \\
\hline Cans & TapeLift & 21 & 10 & 34 \\
\hline Cans & TapeLift & 21 & 11 & 57 \\
\hline
\end{tabular}




\begin{tabular}{|c|c|c|c|c|}
\hline Cans & TapeLift & 21 & 12 & 80 \\
\hline Cans & TapeLift & 21 & 13 & 57 \\
\hline Cans & TapeLift & 21 & 14 & 40 \\
\hline Cans & TapeLift & 21 & 15 & 49 \\
\hline Cans & TapeLift & 21 & 16 & 42 \\
\hline Cans & TapeLift & 21 & 17 & 43 \\
\hline Cans & TapeLift & 21 & 18 & 40 \\
\hline Cans & TapeLift & 21 & 19 & 58 \\
\hline Cans & TapeLift & 21 & 20 & 35 \\
\hline Cans & TapeLift & 22 & 1 & 30 \\
\hline Cans & TapeLift & 22 & 2 & 34 \\
\hline Cans & TapeLift & 22 & 3 & 24 \\
\hline Cans & TapeLift & 22 & 4 & 40 \\
\hline Cans & TapeLift & 22 & 5 & 22 \\
\hline Cans & TapeLift & 22 & 6 & 30 \\
\hline Cans & TapeLift & 22 & 7 & 45 \\
\hline Cans & TapeLift & 22 & 8 & 52 \\
\hline Cans & TapeLift & 22 & 9 & 22 \\
\hline Cans & TapeLift & 22 & 10 & 47 \\
\hline Cans & TapeLift & 22 & 11 & 30 \\
\hline Cans & TapeLift & 22 & 12 & 20 \\
\hline Cans & TapeLift & 22 & 13 & 30 \\
\hline Cans & TapeLift & 22 & 14 & 66 \\
\hline Cans & TapeLift & 22 & 15 & 56 \\
\hline Cans & TapeLift & 22 & 16 & 39 \\
\hline Cans & TapeLift & 22 & 17 & 45 \\
\hline Cans & TapeLift & 22 & 18 & 44 \\
\hline Cans & TapeLift & 22 & 19 & 27 \\
\hline Cans & TapeLift & 22 & 20 & 18 \\
\hline Cans & TapeLift & 22 & 21 & 26 \\
\hline Cans & TapeLift & 22 & 22 & 41 \\
\hline Cans & TapeLift & 22 & 23 & 46 \\
\hline Cans & TapeLift & 22 & 24 & 42 \\
\hline Cans & TapeLift & 22 & 25 & 46 \\
\hline Cans & TapeLift & 22 & 26 & 45 \\
\hline Cans & TapeLift & 22 & 27 & 32 \\
\hline Cans & TapeLift & 22 & 28 & 36 \\
\hline Cans & TapeLift & 22 & 29 & 52 \\
\hline Cans & TapeLift & 22 & 30 & 37 \\
\hline Cans & TapeLift & 22 & 31 & 34 \\
\hline Cans & TapeLift & 22 & 32 & 17 \\
\hline Cans & TapeLift & 22 & 33 & 45 \\
\hline Cans & TapeLift & 22 & 34 & 16 \\
\hline Cans & TapeLift & 23 & 1 & 16 \\
\hline Cans & TapeLift & 23 & 2 & 8 \\
\hline
\end{tabular}




\begin{tabular}{|c|c|c|c|}
\hline Cans & TapeLift & 23 & 3 \\
\hline Cans & TapeLift & 23 & 4 \\
\hline Cans & TapeLift & 23 & 5 \\
\hline Cans & TapeLift & 23 & 6 \\
\hline Cans & TapeLift & 23 & 7 \\
\hline Cans & TapeLift & 23 & 8 \\
\hline Cans & TapeLift & 23 & 9 \\
\hline Cans & TapeLift & 23 & 10 \\
\hline Cans & TapeLift & 23 & 11 \\
\hline Cans & TapeLift & 23 & 12 \\
\hline Cans & TapeLift & 23 & 13 \\
\hline Cans & TapeLift & 23 & 14 \\
\hline Cans & TapeLift & 23 & 15 \\
\hline Cans & TapeLift & 23 & 16 \\
\hline Cans & TapeLift & 23 & 17 \\
\hline Cans & TapeLift & 23 & 18 \\
\hline Cans & TapeLift & 23 & 19 \\
\hline Cans & TapeLift & 23 & 20 \\
\hline Cans & TapeLift & 23 & 21 \\
\hline Cans & TapeLift & 23 & 22 \\
\hline Cans & TapeLift & 23 & 23 \\
\hline Cans & TapeLift & 23 & 24 \\
\hline Cans & TapeLift & 23 & 25 \\
\hline Cans & TapeLift & 23 & 26 \\
\hline Cans & TapeLift & 23 & 27 \\
\hline Cans & TapeLift & 23 & 28 \\
\hline Cans & TapeLift & 23 & 29 \\
\hline Cans & TapeLift & 23 & 30 \\
\hline Cans & TapeLift & 24 & 1 \\
\hline Cans & TapeLift & 24 & 2 \\
\hline Cans & TapeLift & 24 & 3 \\
\hline Cans & TapeLift & 24 & 4 \\
\hline Cans & TapeLift & 24 & 5 \\
\hline Cans & TapeLift & 24 & 6 \\
\hline Cans & TapeLift & 24 & 7 \\
\hline Cans & TapeLift & 24 & 8 \\
\hline Cans & TapeLift & 24 & 9 \\
\hline Cans & TapeLift & 24 & 10 \\
\hline Cans & TapeLift & 24 & 11 \\
\hline Cans & TapeLift & 24 & 12 \\
\hline Cans & TapeLift & 24 & 13 \\
\hline Cans & TapeLift & 24 & 14 \\
\hline Cans & TapeLift & 24 & 15 \\
\hline Cans & TapeLift & 24 & 16 \\
\hline Cans & TapeLift & 24 & 17 \\
\hline
\end{tabular}




\begin{tabular}{|c|c|c|c|}
\hline Cans & TapeLift & 24 & 18 \\
\hline Cans & TapeLift & 24 & 19 \\
\hline Cans & TapeLift & 24 & 20 \\
\hline Cans & TapeLift & 24 & 21 \\
\hline Cans & TapeLift & 24 & 22 \\
\hline Cans & TapeLift & 24 & 23 \\
\hline Cans & TapeLift & 24 & 24 \\
\hline Cans & TapeLift & 24 & 25 \\
\hline Cans & TapeLift & 25 & 1 \\
\hline Cans & TapeLift & 25 & 2 \\
\hline Cans & TapeLift & 25 & 3 \\
\hline Cans & TapeLift & 25 & 4 \\
\hline Cans & TapeLift & 25 & 5 \\
\hline Cans & TapeLift & 25 & 6 \\
\hline Cans & TapeLift & 25 & 7 \\
\hline Cans & TapeLift & 25 & 8 \\
\hline Cans & TapeLift & 25 & 9 \\
\hline Cans & TapeLift & 25 & 10 \\
\hline Cans & TapeLift & 25 & 11 \\
\hline Cans & TapeLift & 25 & 12 \\
\hline Cans & TapeLift & 25 & 13 \\
\hline Cans & TapeLift & 25 & 14 \\
\hline Cans & TapeLift & 25 & 15 \\
\hline Cans & TapeLift & 25 & 16 \\
\hline Cans & TapeLift & 25 & 17 \\
\hline Cans & TapeLift & 25 & 18 \\
\hline Cans & TapeLift & 25 & 19 \\
\hline Cans & TapeLift & 25 & 20 \\
\hline Cans & TapeLift & 25 & 21 \\
\hline Cans & TapeLift & 25 & 22 \\
\hline Cans & TapeLift & 25 & 23 \\
\hline Cans & TapeLift & 25 & 24 \\
\hline Cans & TapeLift & 25 & 25 \\
\hline Cans & TapeLift & 25 & 26 \\
\hline Cans & TapeLift & 25 & 27 \\
\hline Cans & TapeLift & 25 & 28 \\
\hline Cans & TapeLift & 25 & 29 \\
\hline Cans & TapeLift & 25 & 30 \\
\hline Cans & TapeLift & 25 & 31 \\
\hline Cans & TapeLift & 25 & 32 \\
\hline Cans & TapeLift & 26 & 1 \\
\hline Cans & TapeLift & 26 & 2 \\
\hline Cans & TapeLift & 26 & 3 \\
\hline Cans & TapeLift & 26 & 4 \\
\hline Cans & TapeLift & 26 & 5 \\
\hline
\end{tabular}




\begin{tabular}{|c|c|c|c|c|}
\hline Cans & TapeLift & 26 & 6 & 32 \\
\hline Cans & TapeLift & 26 & 7 & 51 \\
\hline Cans & TapeLift & 26 & 8 & 33 \\
\hline Cans & TapeLift & 26 & 9 & 39 \\
\hline Cans & TapeLift & 26 & 10 & 21 \\
\hline Cans & TapeLift & 26 & 11 & 51 \\
\hline Cans & TapeLift & 26 & 12 & 32 \\
\hline Cans & TapeLift & 26 & 13 & 31 \\
\hline Cans & TapeLift & 26 & 14 & 30 \\
\hline Cans & TapeLift & 26 & 15 & 40 \\
\hline Cans & TapeLift & 26 & 16 & 39 \\
\hline Cans & TapeLift & 26 & 17 & 36 \\
\hline Cans & TapeLift & 26 & 18 & 31 \\
\hline Cans & TapeLift & 26 & 19 & 36 \\
\hline Cans & TapeLift & 26 & 20 & 56 \\
\hline Cans & TapeLift & 26 & 21 & 46 \\
\hline Cans & TapeLift & 26 & 22 & 53 \\
\hline Cans & TapeLift & 26 & 23 & 40 \\
\hline Cans & TapeLift & 26 & 24 & 15 \\
\hline Cans & TapeLift & 26 & 25 & 21 \\
\hline Cans & TapeLift & 26 & 26 & 20 \\
\hline Cans & TapeLift & 26 & 27 & 9 \\
\hline Cans & TapeLift & 26 & 28 & 75 \\
\hline Cans & TapeLift & 26 & 29 & 124 \\
\hline Cans & TapeLift & 26 & 30 & 65 \\
\hline Cans & TapeLift & 26 & 31 & 25 \\
\hline Cans & TapeLift & 26 & 32 & 39 \\
\hline Cans & TapeLift & 26 & 33 & 35 \\
\hline Cans & TapeLift & 26 & 34 & 24 \\
\hline Cans & TapeLift & 26 & 35 & 68 \\
\hline Cans & TapeLift & 26 & 36 & 36 \\
\hline Cans & TapeLift & 26 & 37 & 29 \\
\hline Cans & TapeLift & 26 & 38 & 29 \\
\hline Cans & TapeLift & 26 & 39 & 46 \\
\hline Cans & TapeLift & 26 & 40 & 32 \\
\hline Cans & TapeLift & 26 & 41 & 59 \\
\hline Cans & TapeLift & 26 & 42 & 43 \\
\hline Cans & TapeLift & 26 & 43 & 63 \\
\hline Cans & TapeLift & 26 & 44 & 26 \\
\hline Cans & TapeLift & 26 & 45 & 72 \\
\hline Cans & TapeLift & 27 & 1 & 41 \\
\hline Cans & TapeLift & 27 & 2 & 47 \\
\hline Cans & TapeLift & 27 & 3 & 44 \\
\hline Cans & TapeLift & 27 & 4 & 33 \\
\hline Cans & TapeLift & 27 & 5 & 38 \\
\hline
\end{tabular}




\begin{tabular}{|c|c|c|c|c|}
\hline Cans & TapeLift & 27 & 6 & 67 \\
\hline Cans & TapeLift & 27 & 7 & 37 \\
\hline Cans & TapeLift & 27 & 8 & 74 \\
\hline Cans & TapeLift & 27 & 9 & 37 \\
\hline Cans & TapeLift & 27 & 10 & 37 \\
\hline Cans & TapeLift & 27 & 11 & 48 \\
\hline Cans & TapeLift & 27 & 12 & 22 \\
\hline Cans & TapeLift & 27 & 13 & 20 \\
\hline Cans & TapeLift & 27 & 14 & 37 \\
\hline Cans & TapeLift & 27 & 15 & 37 \\
\hline Cans & TapeLift & 27 & 16 & 81 \\
\hline Cans & TapeLift & 27 & 17 & 47 \\
\hline Cans & TapeLift & 27 & 18 & 60 \\
\hline Cans & TapeLift & 27 & 19 & 31 \\
\hline Cans & TapeLift & 27 & 20 & 142 \\
\hline Cans & TapeLift & 27 & 21 & 77 \\
\hline Cans & TapeLift & 27 & 22 & 11 \\
\hline Cans & TapeLift & 27 & 23 & 63 \\
\hline Cans & TapeLift & 27 & 24 & 56 \\
\hline Cans & TapeLift & 28 & 1 & 45 \\
\hline Cans & TapeLift & 28 & 2 & 50 \\
\hline Cans & TapeLift & 28 & 3 & 55 \\
\hline Cans & TapeLift & 28 & 4 & 24 \\
\hline Cans & TapeLift & 28 & 5 & 25 \\
\hline Cans & TapeLift & 28 & 6 & 24 \\
\hline Cans & TapeLift & 28 & 7 & 23 \\
\hline Cans & TapeLift & 28 & 8 & 44 \\
\hline Cans & TapeLift & 28 & 9 & 4 \\
\hline Cans & TapeLift & 28 & 10 & 38 \\
\hline Cans & TapeLift & 28 & 11 & 28 \\
\hline Cans & TapeLift & 28 & 12 & 44 \\
\hline Cans & TapeLift & 28 & 13 & 78 \\
\hline Cans & TapeLift & 28 & 14 & 60 \\
\hline Cans & TapeLift & 28 & 15 & 42 \\
\hline Cans & TapeLift & 28 & 16 & 14 \\
\hline Cans & TapeLift & 28 & 17 & 32 \\
\hline Cans & TapeLift & 28 & 18 & 9 \\
\hline Cans & TapeLift & 28 & 19 & 34 \\
\hline Cans & TapeLift & 28 & 20 & 21 \\
\hline Cans & TapeLift & 28 & 21 & 13 \\
\hline Cans & TapeLift & 28 & 22 & 88 \\
\hline Cans & TapeLift & 28 & 23 & 45 \\
\hline Cans & TapeLift & 28 & 24 & 47 \\
\hline Cans & TapeLift & 28 & 25 & 30 \\
\hline Cans & TapeLift & 28 & 26 & 20 \\
\hline
\end{tabular}




\begin{tabular}{|c|c|c|c|c|}
\hline Cans & TapeLift & 28 & 27 & 40 \\
\hline Cans & TapeLift & 28 & 28 & 43 \\
\hline Cans & TapeLift & 29 & 1 & 49 \\
\hline Cans & TapeLift & 29 & 2 & 53 \\
\hline Cans & TapeLift & 29 & 3 & 78 \\
\hline Cans & TapeLift & 29 & 4 & 24 \\
\hline Cans & TapeLift & 29 & 5 & 72 \\
\hline Cans & TapeLift & 29 & 6 & 48 \\
\hline Cans & TapeLift & 29 & 7 & 48 \\
\hline Cans & TapeLift & 29 & 8 & 50 \\
\hline Cans & TapeLift & 29 & 9 & 31 \\
\hline Cans & TapeLift & 29 & 10 & 56 \\
\hline Cans & TapeLift & 29 & 11 & 44 \\
\hline Cans & TapeLift & 29 & 12 & 52 \\
\hline Cans & TapeLift & 29 & 13 & 29 \\
\hline Cans & TapeLift & 29 & 14 & 73 \\
\hline Cans & TapeLift & 29 & 15 & 50 \\
\hline Cans & TapeLift & 29 & 16 & 56 \\
\hline Cans & TapeLift & 29 & 17 & 33 \\
\hline Cans & TapeLift & 29 & 18 & 57 \\
\hline Cans & TapeLift & 29 & 19 & 36 \\
\hline Cans & TapeLift & 29 & 20 & 59 \\
\hline Cans & TapeLift & 30 & 1 & 52 \\
\hline Cans & TapeLift & 30 & 2 & 65 \\
\hline Cans & TapeLift & 30 & 3 & 32 \\
\hline Cans & TapeLift & 30 & 4 & 22 \\
\hline Cans & TapeLift & 30 & 5 & 45 \\
\hline Cans & TapeLift & 30 & 6 & 26 \\
\hline Cans & TapeLift & 30 & 7 & 42 \\
\hline Cans & TapeLift & 30 & 8 & 77 \\
\hline Cans & TapeLift & 30 & 9 & 68 \\
\hline Cans & TapeLift & 30 & 10 & 32 \\
\hline Cans & TapeLift & 30 & 11 & 28 \\
\hline Cans & TapeLift & 30 & 12 & 50 \\
\hline Cans & TapeLift & 30 & 13 & 35 \\
\hline Cans & TapeLift & 30 & 14 & 37 \\
\hline Cans & TapeLift & 30 & 15 & 84 \\
\hline Cans & TapeLift & 30 & 16 & 87 \\
\hline Cans & TapeLift & 30 & 17 & 56 \\
\hline Cans & TapeLift & 30 & 18 & 37 \\
\hline Cans & TapeLift & 30 & 19 & 50 \\
\hline Cans & TapeLift & 30 & 20 & 37 \\
\hline Cans & TapeLift & 30 & 21 & 20 \\
\hline Cans & TapeLift & 30 & 22 & 37 \\
\hline Cans & TapeLift & 31 & 1 & 79 \\
\hline
\end{tabular}




\begin{tabular}{|c|c|c|c|}
\hline Cans & TapeLift & 31 & 2 \\
\hline Cans & TapeLift & 31 & 3 \\
\hline Cans & TapeLift & 31 & 4 \\
\hline Cans & TapeLift & 31 & 5 \\
\hline Cans & TapeLift & 31 & 6 \\
\hline Cans & TapeLift & 31 & 7 \\
\hline Cans & TapeLift & 31 & 8 \\
\hline Cans & TapeLift & 31 & 9 \\
\hline Cans & TapeLift & 31 & 10 \\
\hline Cans & TapeLift & 31 & 11 \\
\hline Cans & TapeLift & 31 & 12 \\
\hline Cans & TapeLift & 31 & 13 \\
\hline Cans & TapeLift & 31 & 14 \\
\hline Cans & TapeLift & 31 & 15 \\
\hline Cans & TapeLift & 31 & 16 \\
\hline Cans & TapeLift & 31 & 17 \\
\hline Cans & TapeLift & 32 & 1 \\
\hline Cans & TapeLift & 32 & 2 \\
\hline Cans & TapeLift & 32 & 3 \\
\hline Cans & TapeLift & 32 & 4 \\
\hline Cans & TapeLift & 32 & 5 \\
\hline Cans & TapeLift & 32 & 6 \\
\hline Cans & TapeLift & 32 & 7 \\
\hline Cans & TapeLift & 32 & 8 \\
\hline Cans & TapeLift & 32 & 9 \\
\hline Cans & TapeLift & 32 & 10 \\
\hline Cans & TapeLift & 32 & 11 \\
\hline Cans & TapeLift & 32 & 12 \\
\hline Cans & TapeLift & 32 & 13 \\
\hline Cans & TapeLift & 32 & 14 \\
\hline Cans & TapeLift & 32 & 15 \\
\hline Cans & TapeLift & 32 & 16 \\
\hline Cans & TapeLift & 32 & 17 \\
\hline Cans & TapeLift & 32 & 18 \\
\hline Cans & TapeLift & 32 & 19 \\
\hline Cans & TapeLift & 32 & 20 \\
\hline Cans & TapeLift & 32 & 21 \\
\hline Cans & TapeLift & 32 & 22 \\
\hline Cans & TapeLift & 32 & 23 \\
\hline Cans & TapeLift & 32 & 24 \\
\hline Cans & TapeLift & 32 & 25 \\
\hline Cans & TapeLift & 33 & 1 \\
\hline Cans & TapeLift & 33 & 2 \\
\hline Cans & TapeLift & 33 & 3 \\
\hline Cans & TapeLift & 33 & 4 \\
\hline
\end{tabular}




\begin{tabular}{|c|c|c|c|c|}
\hline Cans & TapeLift & 33 & 5 & 52 \\
\hline Cans & TapeLift & 33 & 6 & 94 \\
\hline Cans & TapeLift & 33 & 7 & 37 \\
\hline Cans & TapeLift & 33 & 8 & 48 \\
\hline Cans & TapeLift & 33 & 9 & 37 \\
\hline Cans & TapeLift & 33 & 10 & 47 \\
\hline Cans & TapeLift & 33 & 11 & 43 \\
\hline Cans & TapeLift & 33 & 12 & 55 \\
\hline Cans & TapeLift & 33 & 13 & 47 \\
\hline Cans & TapeLift & 33 & 14 & 45 \\
\hline Cans & TapeLift & 33 & 15 & 31 \\
\hline Cans & TapeLift & 33 & 16 & 37 \\
\hline Cans & TapeLift & 33 & 17 & 39 \\
\hline Cans & TapeLift & 33 & 18 & 29 \\
\hline Cans & TapeLift & 33 & 19 & 42 \\
\hline Cans & TapeLift & 33 & 20 & 61 \\
\hline Cans & TapeLift & 33 & 21 & 57 \\
\hline Cans & TapeLift & 33 & 22 & 41 \\
\hline Cans & TapeLift & 33 & 23 & 57 \\
\hline Cans & TapeLift & 33 & 24 & 46 \\
\hline Cans & TapeLift & 33 & 25 & 31 \\
\hline Cans & TapeLift & 33 & 26 & 33 \\
\hline Cans & TapeLift & 33 & 27 & 31 \\
\hline Cans & TapeLift & 33 & 28 & 49 \\
\hline Cans & TapeLift & 33 & 29 & 34 \\
\hline Cans & TapeLift & 33 & 30 & 39 \\
\hline Cans & TapeLift & 34 & 1 & 30 \\
\hline Cans & TapeLift & 34 & 2 & 54 \\
\hline Cans & TapeLift & 34 & 3 & 24 \\
\hline Cans & TapeLift & 34 & 4 & 42 \\
\hline Cans & TapeLift & 34 & 5 & 38 \\
\hline Cans & TapeLift & 34 & 6 & 41 \\
\hline Cans & TapeLift & 34 & 7 & 33 \\
\hline Cans & TapeLift & 34 & 8 & 73 \\
\hline Cans & TapeLift & 34 & 9 & 21 \\
\hline Cans & TapeLift & 34 & 10 & 72 \\
\hline Cans & TapeLift & 34 & 11 & 15 \\
\hline Cans & TapeLift & 34 & 12 & 43 \\
\hline Cans & TapeLift & 34 & 13 & 32 \\
\hline Cans & TapeLift & 34 & 14 & 38 \\
\hline Cans & TapeLift & 34 & 15 & 37 \\
\hline Cans & TapeLift & 34 & 16 & 31 \\
\hline Cans & TapeLift & 34 & 17 & 39 \\
\hline Cans & TapeLift & 34 & 18 & 31 \\
\hline Cans & TapeLift & 34 & 19 & 23 \\
\hline
\end{tabular}




\begin{tabular}{|c|c|c|c|c|}
\hline Cans & TapeLift & 34 & 20 & 40 \\
\hline Cans & TapeLift & 34 & 21 & 32 \\
\hline Cans & TapeLift & 34 & 22 & 24 \\
\hline Cans & TapeLift & 34 & 23 & 39 \\
\hline Cans & TapeLift & 35 & 1 & 13 \\
\hline Cans & TapeLift & 35 & 2 & 37 \\
\hline Cans & TapeLift & 35 & 3 & 34 \\
\hline Cans & TapeLift & 35 & 4 & 39 \\
\hline Cans & TapeLift & 35 & 5 & 27 \\
\hline Cans & TapeLift & 35 & 6 & 40 \\
\hline Cans & TapeLift & 35 & 7 & 56 \\
\hline Cans & TapeLift & 35 & 8 & 70 \\
\hline Cans & TapeLift & 35 & 9 & 30 \\
\hline Cans & TapeLift & 35 & 10 & 31 \\
\hline Cans & TapeLift & 35 & 11 & 56 \\
\hline Cans & TapeLift & 35 & 12 & 17 \\
\hline Cans & TapeLift & 35 & 13 & 36 \\
\hline Cans & TapeLift & 35 & 14 & 22 \\
\hline Cans & TapeLift & 35 & 15 & 20 \\
\hline Cans & TapeLift & 35 & 16 & 48 \\
\hline Cans & TapeLift & 35 & 17 & 39 \\
\hline Cans & TapeLift & 35 & 18 & 53 \\
\hline Cans & TapeLift & 35 & 19 & 44 \\
\hline Cans & TapeLift & 35 & 20 & 36 \\
\hline Cans & TapeLift & 35 & 21 & 49 \\
\hline Cans & TapeLift & 35 & 22 & 66 \\
\hline Cans & Vacuum & 1 & 1 & 40 \\
\hline Cans & Vacuum & 1 & 2 & 36 \\
\hline Cans & Vacuum & 1 & 3 & 34 \\
\hline Cans & Vacuum & 1 & 4 & 58 \\
\hline Cans & Vacuum & 1 & 5 & 37 \\
\hline Cans & Vacuum & 1 & 6 & 26 \\
\hline Cans & Vacuum & 1 & 7 & 19 \\
\hline Cans & Vacuum & 1 & 8 & 36 \\
\hline Cans & Vacuum & 1 & 9 & 58 \\
\hline Cans & Vacuum & 1 & 10 & 20 \\
\hline Cans & Vacuum & 3 & 1 & 45 \\
\hline Cans & Vacuum & 3 & 2 & 6 \\
\hline Cans & Vacuum & 3 & 3 & 2 \\
\hline Cans & Vacuum & 3 & 4 & 43 \\
\hline Cans & Vacuum & 3 & 5 & 20 \\
\hline Cans & Vacuum & 3 & 6 & 30 \\
\hline Cans & Vacuum & 3 & 7 & 52 \\
\hline Cans & Vacuum & 3 & 8 & 31 \\
\hline Cans & Vacuum & 3 & 9 & 53 \\
\hline
\end{tabular}




\begin{tabular}{|c|c|c|c|c|}
\hline Cans & Vacuum & 3 & 10 & 49 \\
\hline Cans & Vacuum & 4 & 1 & 45 \\
\hline Cans & Vacuum & 4 & 2 & 57 \\
\hline Cans & Vacuum & 4 & 3 & 26 \\
\hline Cans & Vacuum & 4 & 4 & 5 \\
\hline Cans & Vacuum & 4 & 5 & 5 \\
\hline Cans & Vacuum & 4 & 6 & 5 \\
\hline Cans & Vacuum & 4 & 7 & 16 \\
\hline Cans & Vacuum & 4 & 8 & 38 \\
\hline Cans & Vacuum & 4 & 9 & 5 \\
\hline Cans & Vacuum & 4 & 10 & 27 \\
\hline Cans & Vacuum & 5 & 1 & 29 \\
\hline Cans & Vacuum & 5 & 2 & 24 \\
\hline Cans & Vacuum & 5 & 3 & 52 \\
\hline Cans & Vacuum & 5 & 4 & 20 \\
\hline Cans & Vacuum & 5 & 5 & 5 \\
\hline Cans & Vacuum & 5 & 6 & 4 \\
\hline Cans & Vacuum & 5 & 7 & 11 \\
\hline Cans & Vacuum & 5 & 8 & 7 \\
\hline Cans & Vacuum & 5 & 9 & 16 \\
\hline Cans & Vacuum & 5 & 10 & 37 \\
\hline Cans & Vacuum & 5 & 11 & 11 \\
\hline Cans & Vacuum & 5 & 12 & 21 \\
\hline Cans & Vacuum & 6 & 1 & 47 \\
\hline Cans & Vacuum & 6 & 2 & 20 \\
\hline Cans & Vacuum & 6 & 3 & 21 \\
\hline Cans & Vacuum & 6 & 4 & 32 \\
\hline Cans & Vacuum & 6 & 5 & 20 \\
\hline Cans & Vacuum & 6 & 6 & 34 \\
\hline Cans & Vacuum & 6 & 7 & 56 \\
\hline Cans & Vacuum & 6 & 8 & 13 \\
\hline Cans & Vacuum & 6 & 9 & 31 \\
\hline Cans & Vacuum & 6 & 10 & 18 \\
\hline Cans & Vacuum & 6 & 11 & 20 \\
\hline Cans & Vacuum & 6 & 12 & 12 \\
\hline Cans & Vacuum & 6 & 13 & 26 \\
\hline Cans & Vacuum & 7 & 1 & 11 \\
\hline Cans & Vacuum & 7 & 2 & 21 \\
\hline Cans & Vacuum & 7 & 3 & 12 \\
\hline Cans & Vacuum & 8 & 1 & 26 \\
\hline Cans & Vacuum & 8 & 2 & 16 \\
\hline Cans & Vacuum & 8 & 3 & 38 \\
\hline Cans & Vacuum & 8 & 4 & 12 \\
\hline Cans & Vacuum & 8 & 5 & 5 \\
\hline Cans & Vacuum & 8 & 6 & 7 \\
\hline
\end{tabular}




\begin{tabular}{|c|c|c|c|c|}
\hline Cans & Vacuum & 8 & 7 & 10 \\
\hline Cans & Vacuum & 8 & 8 & 16 \\
\hline Cans & Vacuum & 8 & 9 & 3 \\
\hline Cans & Vacuum & 8 & 10 & 35 \\
\hline Cans & Vacuum & 8 & 11 & 9 \\
\hline Cans & Vacuum & 8 & 12 & 1 \\
\hline Cans & Vacuum & 8 & 13 & 69 \\
\hline Cans & Vacuum & 8 & 14 & 70 \\
\hline Cans & Vacuum & 8 & 15 & 49 \\
\hline Cans & Vacuum & 8 & 16 & 43 \\
\hline Cans & Vacuum & 8 & 17 & 57 \\
\hline Cans & Vacuum & 8 & 18 & 47 \\
\hline Cans & Vacuum & 8 & 19 & 47 \\
\hline Cans & Vacuum & 8 & 20 & 76 \\
\hline Cans & Vacuum & 8 & 21 & 76 \\
\hline Cans & Vacuum & 8 & 22 & 57 \\
\hline Cans & Vacuum & 8 & 23 & 50 \\
\hline Cans & Vacuum & 8 & 24 & 54 \\
\hline Cans & Vacuum & 8 & 25 & 24 \\
\hline Cans & Vacuum & 8 & 26 & 45 \\
\hline Cans & Vacuum & 9 & 1 & 24 \\
\hline Cans & Vacuum & 9 & 2 & 54 \\
\hline Cans & Vacuum & 9 & 3 & 23 \\
\hline Cans & Vacuum & 9 & 4 & 12 \\
\hline Cans & Vacuum & 9 & 5 & 20 \\
\hline Cans & Vacuum & 9 & 6 & 34 \\
\hline Cans & Vacuum & 9 & 7 & 47 \\
\hline Cans & Vacuum & 9 & 8 & 43 \\
\hline Cans & Vacuum & 9 & 9 & 31 \\
\hline Cans & Vacuum & 9 & 10 & 18 \\
\hline Cans & Vacuum & 9 & 11 & 26 \\
\hline Cans & Vacuum & 9 & 12 & 62 \\
\hline Cans & Vacuum & 9 & 13 & 54 \\
\hline Cans & Vacuum & 9 & 14 & 17 \\
\hline Cans & Vacuum & 9 & 15 & 35 \\
\hline Cans & Vacuum & 9 & 16 & 20 \\
\hline Cans & Vacuum & 10 & 1 & 28 \\
\hline Cans & Vacuum & 10 & 2 & 42 \\
\hline Cans & Vacuum & 10 & 3 & 13 \\
\hline Cans & Vacuum & 10 & 4 & 3 \\
\hline Cans & Vacuum & 10 & 5 & 4 \\
\hline Cans & Vacuum & 10 & 6 & 7 \\
\hline Cans & Vacuum & 10 & 7 & 11 \\
\hline Cans & Vacuum & 10 & 8 & 1 \\
\hline Cans & Vacuum & 10 & 9 & 16 \\
\hline
\end{tabular}




\begin{tabular}{|c|c|c|c|}
\hline Cans & Vacuum & 10 & 10 \\
\hline Cans & Vacuum & 10 & 11 \\
\hline Cans & Vacuum & 10 & 12 \\
\hline Cans & Vacuum & 10 & 13 \\
\hline Cans & Vacuum & 10 & 14 \\
\hline Cans & Vacuum & 10 & 15 \\
\hline Cans & Vacuum & 10 & 16 \\
\hline Cans & Vacuum & 11 & 1 \\
\hline Cans & Vacuum & 11 & 2 \\
\hline Cans & Vacuum & 11 & 3 \\
\hline Cans & Vacuum & 11 & 4 \\
\hline Cans & Vacuum & 11 & 5 \\
\hline Cans & Vacuum & 11 & 6 \\
\hline Cans & Vacuum & 11 & 7 \\
\hline Cans & Vacuum & 11 & 8 \\
\hline Cans & Vacuum & 11 & 9 \\
\hline Cans & Vacuum & 11 & 10 \\
\hline Cans & Vacuum & 11 & 11 \\
\hline Cans & Vacuum & 11 & 12 \\
\hline Cans & Vacuum & 11 & 13 \\
\hline Cans & Vacuum & 11 & 14 \\
\hline Cans & Vacuum & 11 & 15 \\
\hline Cans & Vacuum & 12 & 1 \\
\hline Cans & Vacuum & 12 & 2 \\
\hline Cans & Vacuum & 12 & 3 \\
\hline Cans & Vacuum & 12 & 4 \\
\hline Cans & Vacuum & 12 & 5 \\
\hline Cans & Vacuum & 12 & 6 \\
\hline Cans & Vacuum & 12 & 7 \\
\hline Cans & Vacuum & 12 & 8 \\
\hline Cans & Vacuum & 12 & 9 \\
\hline Cans & Vacuum & 12 & 10 \\
\hline Cans & Vacuum & 12 & 11 \\
\hline Cans & Vacuum & 12 & 12 \\
\hline Cans & Vacuum & 12 & 13 \\
\hline Cans & Vacuum & 12 & 14 \\
\hline Cans & Vacuum & 12 & 15 \\
\hline Cans & Vacuum & 12 & 16 \\
\hline Cans & Vacuum & 12 & 17 \\
\hline Cans & Vacuum & 12 & 18 \\
\hline Cans & Vacuum & 12 & 19 \\
\hline Cans & Vacuum & 12 & 20 \\
\hline Cans & Vacuum & 13 & 1 \\
\hline Cans & Vacuum & 13 & 2 \\
\hline Cans & Vacuum & 13 & 3 \\
\hline
\end{tabular}




\begin{tabular}{|c|c|c|c|c|}
\hline Cans & Vacuum & 13 & 4 & 7 \\
\hline Cans & Vacuum & 13 & 5 & 3 \\
\hline Cans & Vacuum & 13 & 6 & 18 \\
\hline Cans & Vacuum & 13 & 7 & 7 \\
\hline Cans & Vacuum & 13 & 8 & 50 \\
\hline Cans & Vacuum & 13 & 9 & 11 \\
\hline Cans & Vacuum & 14 & 1 & 14 \\
\hline Cans & Vacuum & 14 & 2 & 33 \\
\hline Cans & Vacuum & 14 & 3 & 21 \\
\hline Cans & Vacuum & 14 & 4 & 45 \\
\hline Cans & Vacuum & 14 & 5 & 61 \\
\hline Cans & Vacuum & 14 & 6 & 80 \\
\hline Cans & Vacuum & 14 & 7 & 82 \\
\hline Cans & Vacuum & 14 & 8 & 81 \\
\hline Cans & Vacuum & 14 & 9 & 71 \\
\hline Cans & Vacuum & 14 & 10 & 31 \\
\hline Cans & Vacuum & 14 & 11 & 76 \\
\hline Cans & Vacuum & 14 & 12 & 63 \\
\hline Cans & Vacuum & 15 & 1 & 17 \\
\hline Cans & Vacuum & 15 & 2 & 14 \\
\hline Cans & Vacuum & 15 & 3 & 70 \\
\hline Cans & Vacuum & 15 & 4 & 20 \\
\hline Cans & Vacuum & 15 & 5 & 39 \\
\hline Cans & Vacuum & 15 & 6 & 29 \\
\hline Cans & Vacuum & 15 & 7 & 38 \\
\hline Cans & Vacuum & 15 & 8 & 32 \\
\hline Cans & Vacuum & 15 & 9 & 26 \\
\hline Cans & Vacuum & 15 & 10 & 18 \\
\hline Cans & Vacuum & 15 & 11 & 23 \\
\hline Cans & Vacuum & 15 & 12 & 27 \\
\hline Cans & Vacuum & 15 & 13 & 35 \\
\hline Cans & Vacuum & 15 & 14 & 33 \\
\hline Cans & Vacuum & 15 & 15 & 27 \\
\hline Cans & Vacuum & 15 & 16 & 25 \\
\hline Cans & Vacuum & 15 & 17 & 14 \\
\hline Cans & Vacuum & 15 & 18 & 11 \\
\hline Cans & Vacuum & 15 & 19 & 14 \\
\hline Cans & Vacuum & 15 & 20 & 14 \\
\hline Cans & Vacuum & 16 & 1 & 7 \\
\hline Cans & Vacuum & 16 & 2 & 17 \\
\hline Cans & Vacuum & 16 & 3 & 18 \\
\hline Cans & Vacuum & 16 & 4 & 17 \\
\hline Cans & Vacuum & 16 & 5 & 22 \\
\hline Cans & Vacuum & 17 & 1 & 13 \\
\hline Cans & Vacuum & 17 & 2 & 15 \\
\hline
\end{tabular}




\begin{tabular}{|c|c|}
\hline Cans & Vacuum \\
\hline Cans & Vacuun \\
\hline Cans & Vacuu \\
\hline Cans & Vacuun \\
\hline Cans & Vacuun \\
\hline Cans & Vacuur \\
\hline Cans & Vacuun \\
\hline Cans & Vacuun \\
\hline Cans & Vacuun \\
\hline Cans & Vacuun \\
\hline Cans & Vacuun \\
\hline Cans & Vacuun \\
\hline Cans & Vacuun \\
\hline Cans & Vacuun \\
\hline Cans & Vacuun \\
\hline Cans & Vacuun \\
\hline Cans & Vacuun \\
\hline Cans & Vacuun \\
\hline Cans & Vacuun \\
\hline Cans & Vacuun \\
\hline Cans & Vacuun \\
\hline Cans & Vacuun \\
\hline Cans & Vacuun \\
\hline Cans & Vacuun \\
\hline Cans & Vacuun \\
\hline Cans & Vacuun \\
\hline Cans & Vacuun \\
\hline Cans & Vacuun \\
\hline Cans & Vacuun \\
\hline Cans & Vacuur \\
\hline Cans & Vacuur \\
\hline Cans & Vacuun \\
\hline Cans & Vacuur \\
\hline Cans & Vacuur \\
\hline Cans & Vacuur \\
\hline Cans & Vacuu \\
\hline Cans & Vacuun \\
\hline Cans & Vacuur \\
\hline Cans & Vacuur \\
\hline Cans & Vacuu \\
\hline Cans & Vacuu \\
\hline Cans & Vacuur \\
\hline Cans & Vacuun \\
\hline Cans & Vacuun \\
\hline Cans & Vacuu \\
\hline
\end{tabular}

\begin{tabular}{|c|c|}
\hline 17 & 3 \\
\hline 17 & 4 \\
\hline 18 & 1 \\
\hline 18 & 2 \\
\hline 18 & 3 \\
\hline 19 & 1 \\
\hline 19 & 2 \\
\hline 19 & 3 \\
\hline 19 & 4 \\
\hline 19 & 5 \\
\hline 19 & 6 \\
\hline 19 & 7 \\
\hline 20 & 1 \\
\hline 20 & 2 \\
\hline 20 & 3 \\
\hline 20 & 4 \\
\hline 20 & 5 \\
\hline 21 & 1 \\
\hline 21 & 2 \\
\hline 21 & 3 \\
\hline 21 & 4 \\
\hline 21 & 5 \\
\hline 21 & 6 \\
\hline 22 & 1 \\
\hline 22 & 2 \\
\hline 22 & 3 \\
\hline 22 & 4 \\
\hline 22 & 5 \\
\hline 23 & 1 \\
\hline 23 & 2 \\
\hline 23 & 3 \\
\hline 23 & 4 \\
\hline 23 & 5 \\
\hline 23 & 6 \\
\hline 23 & 7 \\
\hline 24 & 1 \\
\hline 24 & 2 \\
\hline 24 & 3 \\
\hline 24 & 4 \\
\hline 24 & 5 \\
\hline 24 & 6 \\
\hline 24 & 7 \\
\hline 24 & 8 \\
\hline 24 & 9 \\
\hline 24 & 10 \\
\hline
\end{tabular}




\begin{tabular}{|c|c|c|c|c|}
\hline Cans & Vacuum & 24 & 11 & 13 \\
\hline Cans & Vacuum & 24 & 12 & 4 \\
\hline Cans & Vacuum & 25 & 1 & 1 \\
\hline Cans & Vacuum & 25 & 2 & 3 \\
\hline Cans & Vacuum & 25 & 3 & 4 \\
\hline Cans & Vacuum & 25 & 4 & 27 \\
\hline Cans & Vacuum & 25 & 5 & 18 \\
\hline Cans & Vacuum & 25 & 6 & 13 \\
\hline Cans & Vacuum & 25 & 7 & 58 \\
\hline Cans & Vacuum & 25 & 8 & 15 \\
\hline Cans & Vacuum & 25 & 9 & 65 \\
\hline Cans & Vacuum & 25 & 10 & 41 \\
\hline Cans & Vacuum & 25 & 11 & 22 \\
\hline Cans & Vacuum & 25 & 12 & 67 \\
\hline Cans & Vacuum & 25 & 13 & 58 \\
\hline Cans & Vacuum & 25 & 14 & 21 \\
\hline Cans & Vacuum & 25 & 15 & 14 \\
\hline Cans & Vacuum & 25 & 16 & 11 \\
\hline Cans & Vacuum & 25 & 17 & 20 \\
\hline Cans & Vacuum & 25 & 18 & 7 \\
\hline Cans & Vacuum & 25 & 19 & 8 \\
\hline Cans & Vacuum & 25 & 20 & 22 \\
\hline Cans & Vacuum & 25 & 21 & 22 \\
\hline Cans & Vacuum & 25 & 22 & 34 \\
\hline Cans & Vacuum & 25 & 23 & 7 \\
\hline Cans & Vacuum & 25 & 24 & 22 \\
\hline Cans & Vacuum & 25 & 25 & 5 \\
\hline Cans & Vacuum & 26 & 1 & 20 \\
\hline Cans & Vacuum & 26 & 2 & 35 \\
\hline Cans & Vacuum & 26 & 3 & 16 \\
\hline Cans & Vacuum & 26 & 4 & 18 \\
\hline Cans & Vacuum & 26 & 5 & 16 \\
\hline Cans & Vacuum & 26 & 6 & 35 \\
\hline Cans & Vacuum & 26 & 7 & 20 \\
\hline Cans & Vacuum & 26 & 8 & 16 \\
\hline Cans & Vacuum & 26 & 9 & 38 \\
\hline Cans & Vacuum & 26 & 10 & 8 \\
\hline Cans & Vacuum & 27 & 1 & 33 \\
\hline Cans & Vacuum & 27 & 2 & 28 \\
\hline Cans & Vacuum & 27 & 3 & 7 \\
\hline Cans & Vacuum & 27 & 4 & 28 \\
\hline Cans & Vacuum & 27 & 5 & 26 \\
\hline Cans & Vacuum & 27 & 6 & 19 \\
\hline Cans & Vacuum & 27 & 7 & 38 \\
\hline Cans & Vacuum & 27 & 8 & 17 \\
\hline
\end{tabular}




\begin{tabular}{|c|c|c|c|}
\hline Cans & Vacuum & 27 & 9 \\
\hline Cans & Vacuum & 27 & 10 \\
\hline Cans & Vacuum & 27 & 11 \\
\hline Cans & Vacuum & 27 & 12 \\
\hline Cans & Vacuum & 27 & 13 \\
\hline Cans & Vacuum & 27 & 14 \\
\hline Cans & Vacuum & 27 & 15 \\
\hline Cans & Vacuum & 27 & 16 \\
\hline Cans & Vacuum & 27 & 17 \\
\hline Cans & Vacuum & 28 & 1 \\
\hline Cans & Vacuum & 28 & 2 \\
\hline Cans & Vacuum & 28 & 3 \\
\hline Cans & Vacuum & 28 & 4 \\
\hline Cans & Vacuum & 28 & 5 \\
\hline Cans & Vacuum & 28 & 6 \\
\hline Cans & Vacuum & 28 & 7 \\
\hline Cans & Vacuum & 29 & 1 \\
\hline Cans & Vacuum & 29 & 2 \\
\hline Cans & Vacuum & 29 & 3 \\
\hline Cans & Vacuum & 29 & 4 \\
\hline Cans & Vacuum & 29 & 5 \\
\hline Cans & Vacuum & 29 & 6 \\
\hline Cans & Vacuum & 29 & 7 \\
\hline Cans & Vacuum & 29 & 8 \\
\hline Cans & Vacuum & 30 & 1 \\
\hline Cans & Vacuum & 30 & 2 \\
\hline Cans & Vacuum & 30 & 3 \\
\hline Cans & Vacuum & 30 & 4 \\
\hline Cans & Vacuum & 30 & 5 \\
\hline Cans & Vacuum & 30 & 6 \\
\hline Cans & Vacuum & 30 & 7 \\
\hline Cans & Vacuum & 30 & 8 \\
\hline Cans & Vacuum & 31 & 1 \\
\hline Cans & Vacuum & 31 & 2 \\
\hline Cans & Vacuum & 31 & 3 \\
\hline Cans & Vacuum & 31 & 4 \\
\hline Cans & Vacuum & 31 & 5 \\
\hline Cans & Vacuum & 31 & 6 \\
\hline Cans & Vacuum & 31 & 7 \\
\hline Cans & Vacuum & 31 & 8 \\
\hline Cans & Vacuum & 31 & 9 \\
\hline Cans & Vacuum & 31 & 10 \\
\hline Cans & Vacuum & 31 & 11 \\
\hline Cans & Vacuum & 31 & 12 \\
\hline Cans & Vacuum & 31 & 13 \\
\hline
\end{tabular}




\begin{tabular}{|c|c|c|c|c|}
\hline Cans & Vacuum & 31 & 14 & 11 \\
\hline Cans & Vacuum & 31 & 15 & 6 \\
\hline Cans & Vacuum & 31 & 16 & 20 \\
\hline Cans & Vacuum & 31 & 17 & 2 \\
\hline Cans & Vacuum & 31 & 18 & 29 \\
\hline Cans & Vacuum & 31 & 19 & 15 \\
\hline Cans & Vacuum & 31 & 20 & 22 \\
\hline Cans & Vacuum & 32 & 1 & 24 \\
\hline Cans & Vacuum & 32 & 2 & 20 \\
\hline Cans & Vacuum & 32 & 3 & 15 \\
\hline Cans & Vacuum & 32 & 4 & 9 \\
\hline Cans & Vacuum & 32 & 5 & 8 \\
\hline Cans & Vacuum & 32 & 6 & 10 \\
\hline Cans & Vacuum & 32 & 7 & 14 \\
\hline Cans & Vacuum & 32 & 8 & 5 \\
\hline Cans & Vacuum & 32 & 9 & 143 \\
\hline Cans & Vacuum & 32 & 10 & 13 \\
\hline Cans & Vacuum & 33 & 1 & 10 \\
\hline Cans & Vacuum & 33 & 2 & 12 \\
\hline Cans & Vacuum & 33 & 3 & 20 \\
\hline Cans & Vacuum & 33 & 4 & 2 \\
\hline Cans & Vacuum & 33 & 5 & 9 \\
\hline Cans & Vacuum & 33 & 6 & 22 \\
\hline Cans & Vacuum & 33 & 7 & 6 \\
\hline Cans & Vacuum & 33 & 8 & 15 \\
\hline Cans & Vacuum & 33 & 9 & 6 \\
\hline Cans & Vacuum & 33 & 10 & 15 \\
\hline Cans & Vacuum & 33 & 11 & 18 \\
\hline Cans & Vacuum & 33 & 12 & 14 \\
\hline Cans & Vacuum & 34 & 1 & 10 \\
\hline Cans & Vacuum & 34 & 2 & 5 \\
\hline Cans & Vacuum & 34 & 3 & 5 \\
\hline Cans & Vacuum & 34 & 4 & 11 \\
\hline Cans & Vacuum & 34 & 5 & 6 \\
\hline Cans & Vacuum & 34 & 6 & 17 \\
\hline Cans & Vacuum & 34 & 7 & 1 \\
\hline Cans & Vacuum & 34 & 8 & 22 \\
\hline Cans & Vacuum & 35 & 1 & 2 \\
\hline Cans & Vacuum & 35 & 2 & 7 \\
\hline Cans & Vacuum & 35 & 3 & 4 \\
\hline Cans & Vacuum & 35 & 4 & 16 \\
\hline Cans & Vacuum & 35 & 5 & 3 \\
\hline Cans & Vacuum & 35 & 6 & 8 \\
\hline Cans & Vacuum & 35 & 7 & 11 \\
\hline Cans & Vacuum & 35 & 8 & 8 \\
\hline
\end{tabular}




\begin{tabular}{|c|c|c|c|c|}
\hline Cans & Vacuum & 35 & 9 & 17 \\
\hline Cans & Vacuum & 35 & 10 & 25 \\
\hline Cans & Vacuum & 35 & 11 & 18 \\
\hline Cans & Vacuum & 35 & 12 & 4 \\
\hline Cans & Vacuum & 35 & 13 & 5 \\
\hline Cans & Vacuum & 35 & 14 & 7 \\
\hline Cans & Vacuum & 35 & 15 & 6 \\
\hline Cans & Vacuum & 35 & 16 & 5 \\
\hline Cans & Vacuum & 35 & 17 & 1 \\
\hline Cans & Vacuum & 35 & 18 & 12 \\
\hline Cans & Vacuum & 35 & 19 & 6 \\
\hline Cans & Vacuum & 35 & 20 & 22 \\
\hline Cans & Vacuum & 35 & 21 & 18 \\
\hline Cans & Vacuum & 35 & 22 & 6 \\
\hline Cans & Vacuum & 35 & 23 & 4 \\
\hline Cans & Vacuum & 35 & 24 & 9 \\
\hline Cans & Vacuum & 35 & 25 & 3 \\
\hline Cans & Vacuum & 35 & 26 & 4 \\
\hline FOTL & TapeLift & 1 & 1 & 50 \\
\hline FOTL & TapeLift & 1 & 2 & 68 \\
\hline FOTL & TapeLift & 1 & 3 & 60 \\
\hline FOTL & TapeLift & 1 & 4 & 65 \\
\hline FOTL & TapeLift & 1 & 5 & 96 \\
\hline FOTL & TapeLift & 1 & 6 & 61 \\
\hline FOTL & TapeLift & 1 & 7 & 58 \\
\hline FOTL & TapeLift & 1 & 8 & 58 \\
\hline FOTL & TapeLift & 1 & 9 & 108 \\
\hline FOTL & TapeLift & 1 & 10 & 91 \\
\hline FOTL & TapeLift & 1 & 11 & 73 \\
\hline FOTL & TapeLift & 1 & 12 & 83 \\
\hline FOTL & TapeLift & 1 & 13 & 109 \\
\hline FOTL & TapeLift & 1 & 14 & 123 \\
\hline FOTL & TapeLift & 1 & 15 & 106 \\
\hline FOTL & TapeLift & 1 & 16 & 132 \\
\hline FOTL & TapeLift & 1 & 17 & 112 \\
\hline FOTL & TapeLift & 1 & 18 & 123 \\
\hline FOTL & TapeLift & 1 & 19 & 107 \\
\hline FOTL & TapeLift & 1 & 20 & 33 \\
\hline FOTL & TapeLift & 1 & 21 & 81 \\
\hline FOTL & TapeLift & 1 & 22 & 76 \\
\hline FOTL & TapeLift & 2 & 1 & 73 \\
\hline FOTL & TapeLift & 2 & 2 & 67 \\
\hline FOTL & TapeLift & 2 & 3 & 57 \\
\hline FOTL & TapeLift & 2 & 4 & 83 \\
\hline FOTL & TapeLift & 2 & 5 & 73 \\
\hline
\end{tabular}




\begin{tabular}{|c|c|c|c|c|}
\hline FOTL & TapeLift & 2 & 6 & 65 \\
\hline FOTL & TapeLift & 2 & 7 & 62 \\
\hline FOTL & TapeLift & 2 & 8 & 59 \\
\hline FOTL & TapeLift & 2 & 9 & 65 \\
\hline FOTL & TapeLift & 2 & 10 & 70 \\
\hline FOTL & TapeLift & 2 & 11 & 80 \\
\hline FOTL & TapeLift & 2 & 12 & 79 \\
\hline FOTL & TapeLift & 2 & 13 & 56 \\
\hline FOTL & TapeLift & 2 & 14 & 66 \\
\hline FOTL & TapeLift & 2 & 15 & 61 \\
\hline FOTL & TapeLift & 2 & 16 & 69 \\
\hline FOTL & TapeLift & 2 & 17 & 80 \\
\hline FOTL & TapeLift & 2 & 18 & 72 \\
\hline FOTL & TapeLift & 2 & 19 & 65 \\
\hline FOTL & TapeLift & 2 & 20 & 56 \\
\hline FOTL & TapeLift & 2 & 21 & 89 \\
\hline FOTL & TapeLift & 2 & 22 & 63 \\
\hline FOTL & TapeLift & 2 & 23 & 60 \\
\hline FOTL & TapeLift & 3 & 1 & 60 \\
\hline FOTL & TapeLift & 3 & 2 & 75 \\
\hline FOTL & TapeLift & 3 & 3 & 87 \\
\hline FOTL & TapeLift & 3 & 4 & 62 \\
\hline FOTL & TapeLift & 3 & 5 & 76 \\
\hline FOTL & TapeLift & 3 & 6 & 76 \\
\hline FOTL & TapeLift & 3 & 7 & 50 \\
\hline FOTL & TapeLift & 3 & 8 & 72 \\
\hline FOTL & TapeLift & 3 & 9 & 50 \\
\hline FOTL & TapeLift & 3 & 10 & 104 \\
\hline FOTL & TapeLift & 3 & 11 & 53 \\
\hline FOTL & TapeLift & 3 & 12 & 53 \\
\hline FOTL & TapeLift & 3 & 13 & 77 \\
\hline FOTL & TapeLift & 3 & 14 & 46 \\
\hline FOTL & TapeLift & 3 & 15 & 41 \\
\hline FOTL & TapeLift & 3 & 16 & 42 \\
\hline FOTL & TapeLift & 3 & 17 & 58 \\
\hline FOTL & TapeLift & 3 & 18 & 64 \\
\hline FOTL & TapeLift & 3 & 19 & 81 \\
\hline FOTL & TapeLift & 3 & 20 & 40 \\
\hline FOTL & TapeLift & 3 & 21 & 51 \\
\hline FOTL & TapeLift & 3 & 22 & 67 \\
\hline FOTL & TapeLift & 3 & 23 & 58 \\
\hline FOTL & TapeLift & 3 & 24 & 57 \\
\hline FOTL & TapeLift & 3 & 1 & 53 \\
\hline FOTL & TapeLift & 4 & 2 & 57 \\
\hline FOTL & TapeLift & 4 & 3 & 49 \\
\hline
\end{tabular}




\begin{tabular}{|c|c|c|c|}
\hline FOTL & TapeLift & 4 & 4 \\
\hline FOTL & TapeLift & 4 & 5 \\
\hline FOTL & TapeLift & 4 & 6 \\
\hline FOTL & TapeLift & 4 & 7 \\
\hline FOTL & TapeLift & 4 & 8 \\
\hline FOTL & TapeLift & 4 & 9 \\
\hline FOTL & TapeLift & 4 & 10 \\
\hline FOTL & TapeLift & 4 & 11 \\
\hline FOTL & TapeLift & 4 & 12 \\
\hline FOTL & TapeLift & 4 & 13 \\
\hline FOTL & TapeLift & 4 & 14 \\
\hline FOTL & TapeLift & 4 & 15 \\
\hline FOTL & TapeLift & 4 & 16 \\
\hline FOTL & TapeLift & 4 & 17 \\
\hline FOTL & TapeLift & 4 & 18 \\
\hline FOTL & TapeLift & 4 & 19 \\
\hline FOTL & TapeLift & 4 & 20 \\
\hline FOTL & TapeLift & 4 & 21 \\
\hline FOTL & TapeLift & 4 & 22 \\
\hline FOTL & TapeLift & 4 & 23 \\
\hline FOTL & TapeLift & 4 & 24 \\
\hline FOTL & TapeLift & 4 & 25 \\
\hline FOTL & TapeLift & 4 & 26 \\
\hline FOTL & TapeLift & 5 & 1 \\
\hline FOTL & TapeLift & 5 & 2 \\
\hline FOTL & TapeLift & 5 & 3 \\
\hline FOTL & TapeLift & 5 & 4 \\
\hline FOTL & TapeLift & 5 & 5 \\
\hline FOTL & TapeLift & 5 & 6 \\
\hline FOTL & TapeLift & 5 & 7 \\
\hline FOTL & TapeLift & 5 & 8 \\
\hline FOTL & TapeLift & 5 & 9 \\
\hline FOTL & TapeLift & 5 & 10 \\
\hline FOTL & TapeLift & 5 & 11 \\
\hline FOTL & TapeLift & 5 & 12 \\
\hline FOTL & TapeLift & 5 & 13 \\
\hline FOTL & TapeLift & 5 & 14 \\
\hline FOTL & TapeLift & 5 & 15 \\
\hline FOTL & TapeLift & 5 & 16 \\
\hline FOTL & TapeLift & 5 & 17 \\
\hline FOTL & TapeLift & 5 & 18 \\
\hline FOTL & TapeLift & 5 & 19 \\
\hline FOTL & TapeLift & 5 & 20 \\
\hline FOTL & TapeLift & 5 & 21 \\
\hline FOTL & TapeLift & 5 & 22 \\
\hline
\end{tabular}




\begin{tabular}{|c|c|c|c|c|}
\hline FOTL & TapeLift & 5 & 23 & 67 \\
\hline FOTL & TapeLift & 5 & 24 & 43 \\
\hline FOTL & TapeLift & 6 & 1 & 47 \\
\hline FOTL & TapeLift & 6 & 2 & 74 \\
\hline FOTL & TapeLift & 6 & 3 & 40 \\
\hline FOTL & TapeLift & 6 & 4 & 40 \\
\hline FOTL & TapeLift & 6 & 5 & 52 \\
\hline FOTL & TapeLift & 6 & 6 & 50 \\
\hline FOTL & TapeLift & 6 & 7 & 62 \\
\hline FOTL & TapeLift & 6 & 8 & 77 \\
\hline FOTL & TapeLift & 6 & 9 & 59 \\
\hline FOTL & TapeLift & 6 & 10 & 46 \\
\hline FOTL & TapeLift & 6 & 11 & 76 \\
\hline FOTL & TapeLift & 6 & 12 & 59 \\
\hline FOTL & TapeLift & 6 & 13 & 90 \\
\hline FOTL & TapeLift & 6 & 14 & 53 \\
\hline FOTL & TapeLift & 6 & 15 & 81 \\
\hline FOTL & TapeLift & 6 & 16 & 90 \\
\hline FOTL & TapeLift & 6 & 17 & 92 \\
\hline FOTL & TapeLift & 6 & 18 & 75 \\
\hline FOTL & TapeLift & 6 & 19 & 50 \\
\hline FOTL & TapeLift & 6 & 20 & 81 \\
\hline FOTL & TapeLift & 6 & 21 & 36 \\
\hline FOTL & TapeLift & 6 & 22 & 84 \\
\hline FOTL & TapeLift & 6 & 23 & 76 \\
\hline FOTL & TapeLift & 6 & 24 & 76 \\
\hline FOTL & TapeLift & 6 & 25 & 66 \\
\hline FOTL & TapeLift & 6 & 26 & 81 \\
\hline FOTL & TapeLift & 6 & 27 & 73 \\
\hline FOTL & TapeLift & 6 & 28 & 76 \\
\hline FOTL & TapeLift & 6 & 29 & 72 \\
\hline FOTL & TapeLift & 6 & 30 & 73 \\
\hline FOTL & TapeLift & 6 & 31 & 91 \\
\hline FOTL & TapeLift & 6 & 32 & 35 \\
\hline FOTL & TapeLift & 6 & 33 & 82 \\
\hline FOTL & TapeLift & 7 & 1 & 64 \\
\hline FOTL & TapeLift & 7 & 2 & 60 \\
\hline FOTL & TapeLift & 7 & 3 & 66 \\
\hline FOTL & TapeLift & 7 & 4 & 82 \\
\hline FOTL & TapeLift & 7 & 5 & 90 \\
\hline FOTL & TapeLift & 7 & 6 & 94 \\
\hline FOTL & TapeLift & 7 & 7 & 57 \\
\hline FOTL & TapeLift & 7 & 8 & 77 \\
\hline FOTL & TapeLift & 7 & 9 & 70 \\
\hline FOTL & TapeLift & 7 & 10 & 51 \\
\hline
\end{tabular}




\begin{tabular}{|c|c|c|c|c|}
\hline FOTL & TapeLift & 7 & 11 & 85 \\
\hline FOTL & TapeLift & 7 & 12 & 89 \\
\hline FOTL & TapeLift & 7 & 13 & 87 \\
\hline FOTL & TapeLift & 7 & 14 & 67 \\
\hline FOTL & TapeLift & 7 & 15 & 65 \\
\hline FOTL & TapeLift & 7 & 16 & 66 \\
\hline FOTL & TapeLift & 7 & 17 & 72 \\
\hline FOTL & TapeLift & 7 & 18 & 54 \\
\hline FOTL & TapeLift & 7 & 19 & 87 \\
\hline FOTL & TapeLift & 7 & 20 & 55 \\
\hline FOTL & TapeLift & 7 & 21 & 54 \\
\hline FOTL & TapeLift & 7 & 22 & 66 \\
\hline FOTL & TapeLift & 7 & 23 & 95 \\
\hline FOTL & TapeLift & 7 & 24 & 61 \\
\hline FOTL & TapeLift & 7 & 25 & 71 \\
\hline FOTL & TapeLift & 7 & 26 & 90 \\
\hline FOTL & TapeLift & 7 & 27 & 64 \\
\hline FOTL & TapeLift & 7 & 28 & 24 \\
\hline FOTL & TapeLift & 7 & 29 & 60 \\
\hline FOTL & TapeLift & 7 & 30 & 56 \\
\hline FOTL & TapeLift & 7 & 31 & 35 \\
\hline FOTL & TapeLift & 7 & 32 & 54 \\
\hline FOTL & TapeLift & 7 & 33 & 64 \\
\hline FOTL & TapeLift & 7 & 34 & 24 \\
\hline FOTL & TapeLift & 7 & 35 & 38 \\
\hline FOTL & TapeLift & 7 & 36 & 66 \\
\hline FOTL & TapeLift & 7 & 37 & 61 \\
\hline FOTL & TapeLift & 7 & 38 & 59 \\
\hline FOTL & TapeLift & 7 & 39 & 23 \\
\hline FOTL & TapeLift & 7 & 40 & 43 \\
\hline FOTL & TapeLift & 7 & 41 & 60 \\
\hline FOTL & TapeLift & 7 & 42 & 42 \\
\hline FOTL & TapeLift & 7 & 43 & 84 \\
\hline FOTL & TapeLift & 7 & 44 & 19 \\
\hline FOTL & TapeLift & 7 & 45 & 54 \\
\hline FOTL & TapeLift & 7 & 46 & 65 \\
\hline FOTL & TapeLift & 7 & 47 & 65 \\
\hline FOTL & TapeLift & 7 & 48 & 49 \\
\hline FOTL & TapeLift & 7 & 49 & 59 \\
\hline FOTL & TapeLift & 7 & 50 & 46 \\
\hline FOTL & TapeLift & 7 & 51 & 56 \\
\hline FOTL & TapeLift & 7 & 52 & 81 \\
\hline FOTL & TapeLift & 7 & 53 & 48 \\
\hline FOTL & TapeLift & 7 & 54 & 111 \\
\hline FOTL & TapeLift & 7 & 55 & 122 \\
\hline
\end{tabular}




\begin{tabular}{|c|c|c|c|c|}
\hline FOTL & TapeLift & 7 & 56 & 89 \\
\hline FOTL & TapeLift & 7 & 57 & 54 \\
\hline FOTL & TapeLift & 7 & 58 & 37 \\
\hline FOTL & TapeLift & 7 & 59 & 113 \\
\hline FOTL & TapeLift & 7 & 60 & 64 \\
\hline FOTL & TapeLift & 7 & 61 & 46 \\
\hline FOTL & TapeLift & 7 & 62 & 98 \\
\hline FOTL & TapeLift & 7 & 63 & 106 \\
\hline FOTL & TapeLift & 7 & 64 & 64 \\
\hline FOTL & TapeLift & 7 & 65 & 43 \\
\hline FOTL & TapeLift & 7 & 66 & 70 \\
\hline FOTL & TapeLift & 7 & 67 & 97 \\
\hline FOTL & TapeLift & 7 & 68 & 64 \\
\hline FOTL & TapeLift & 7 & 69 & 75 \\
\hline FOTL & TapeLift & 7 & 70 & 77 \\
\hline FOTL & TapeLift & 7 & 71 & 73 \\
\hline FOTL & TapeLift & 8 & 1 & 70 \\
\hline FOTL & TapeLift & 8 & 2 & 94 \\
\hline FOTL & TapeLift & 8 & 3 & 100 \\
\hline FOTL & TapeLift & 8 & 4 & 89 \\
\hline FOTL & TapeLift & 8 & 5 & 112 \\
\hline FOTL & TapeLift & 8 & 6 & 90 \\
\hline FOTL & TapeLift & 8 & 7 & 70 \\
\hline FOTL & TapeLift & 8 & 8 & 85 \\
\hline FOTL & TapeLift & 8 & 9 & 129 \\
\hline FOTL & TapeLift & 8 & 10 & 118 \\
\hline FOTL & TapeLift & 8 & 11 & 105 \\
\hline FOTL & TapeLift & 8 & 12 & 128 \\
\hline FOTL & TapeLift & 8 & 13 & 114 \\
\hline FOTL & TapeLift & 8 & 14 & 141 \\
\hline FOTL & TapeLift & 8 & 15 & 125 \\
\hline FOTL & TapeLift & 8 & 16 & 132 \\
\hline FOTL & TapeLift & 8 & 17 & 145 \\
\hline FOTL & TapeLift & 8 & 18 & 110 \\
\hline FOTL & TapeLift & 8 & 19 & 118 \\
\hline FOTL & TapeLift & 8 & 20 & 97 \\
\hline FOTL & TapeLift & 8 & 21 & 125 \\
\hline FOTL & TapeLift & 8 & 22 & 122 \\
\hline FOTL & TapeLift & 8 & 23 & 140 \\
\hline FOTL & TapeLift & 8 & 24 & 73 \\
\hline FOTL & TapeLift & 8 & 25 & 129 \\
\hline FOTL & TapeLift & 8 & 26 & 128 \\
\hline FOTL & TapeLift & 8 & 27 & 139 \\
\hline FOTL & TapeLift & 8 & 28 & 141 \\
\hline FOTL & TapeLift & 8 & 29 & 127 \\
\hline
\end{tabular}




\begin{tabular}{|c|c|c|c|c|}
\hline FOTL & TapeLift & 8 & 30 & 127 \\
\hline FOTL & TapeLift & 8 & 31 & 130 \\
\hline FOTL & TapeLift & 8 & 32 & 135 \\
\hline FOTL & TapeLift & 8 & 33 & 119 \\
\hline FOTL & TapeLift & 8 & 34 & 139 \\
\hline FOTL & TapeLift & 8 & 35 & 83 \\
\hline FOTL & TapeLift & 8 & 36 & 118 \\
\hline FOTL & TapeLift & 8 & 37 & 129 \\
\hline FOTL & TapeLift & 8 & 38 & 115 \\
\hline FOTL & TapeLift & 8 & 39 & 110 \\
\hline FOTL & TapeLift & 8 & 40 & 81 \\
\hline FOTL & TapeLift & 8 & 41 & 83 \\
\hline FOTL & TapeLift & 9 & 1 & 120 \\
\hline FOTL & TapeLift & 9 & 2 & 117 \\
\hline FOTL & TapeLift & 9 & 3 & 104 \\
\hline FOTL & TapeLift & 9 & 4 & 105 \\
\hline FOTL & TapeLift & 9 & 5 & 123 \\
\hline FOTL & TapeLift & 9 & 6 & 106 \\
\hline FOTL & TapeLift & 9 & 7 & 89 \\
\hline FOTL & TapeLift & 9 & 8 & 100 \\
\hline FOTL & TapeLift & 9 & 9 & 104 \\
\hline FOTL & TapeLift & 9 & 10 & 113 \\
\hline FOTL & TapeLift & 9 & 11 & 92 \\
\hline FOTL & TapeLift & 9 & 12 & 89 \\
\hline FOTL & TapeLift & 9 & 13 & 97 \\
\hline FOTL & TapeLift & 9 & 14 & 120 \\
\hline FOTL & TapeLift & 9 & 15 & 128 \\
\hline FOTL & TapeLift & 9 & 16 & 121 \\
\hline FOTL & TapeLift & 9 & 17 & 100 \\
\hline FOTL & TapeLift & 9 & 18 & 98 \\
\hline FOTL & TapeLift & 9 & 19 & 122 \\
\hline FOTL & TapeLift & 9 & 20 & 121 \\
\hline FOTL & TapeLift & 9 & 21 & 119 \\
\hline FOTL & TapeLift & 9 & 22 & 75 \\
\hline FOTL & TapeLift & 9 & 23 & 124 \\
\hline FOTL & TapeLift & 9 & 24 & 119 \\
\hline FOTL & TapeLift & 9 & 25 & 95 \\
\hline FOTL & TapeLift & 9 & 26 & 117 \\
\hline FOTL & TapeLift & 9 & 27 & 88 \\
\hline FOTL & TapeLift & 9 & 28 & 91 \\
\hline FOTL & TapeLift & 9 & 29 & 89 \\
\hline FOTL & TapeLift & 9 & 30 & 109 \\
\hline FOTL & TapeLift & 9 & 31 & 57 \\
\hline FOTL & TapeLift & 9 & 32 & 77 \\
\hline FOTL & TapeLift & 10 & 1 & 76 \\
\hline
\end{tabular}




\begin{tabular}{|c|c|c|c|c|}
\hline FOTL & TapeLift & 10 & 2 & 66 \\
\hline FOTL & TapeLift & 10 & 3 & 103 \\
\hline FOTL & TapeLift & 10 & 4 & 92 \\
\hline FOTL & TapeLift & 10 & 5 & 71 \\
\hline FOTL & TapeLift & 10 & 6 & 67 \\
\hline FOTL & TapeLift & 10 & 7 & 72 \\
\hline FOTL & TapeLift & 10 & 8 & 48 \\
\hline FOTL & TapeLift & 10 & 9 & 72 \\
\hline FOTL & TapeLift & 10 & 10 & 47 \\
\hline FOTL & TapeLift & 10 & 11 & 82 \\
\hline FOTL & TapeLift & 10 & 12 & 84 \\
\hline FOTL & TapeLift & 10 & 13 & 73 \\
\hline FOTL & TapeLift & 10 & 14 & 63 \\
\hline FOTL & TapeLift & 10 & 15 & 62 \\
\hline FOTL & TapeLift & 10 & 16 & 48 \\
\hline FOTL & TapeLift & 10 & 17 & 49 \\
\hline FOTL & TapeLift & 10 & 18 & 66 \\
\hline FOTL & TapeLift & 10 & 19 & 61 \\
\hline FOTL & TapeLift & 10 & 20 & 60 \\
\hline FOTL & TapeLift & 10 & 21 & 73 \\
\hline FOTL & TapeLift & 10 & 22 & 61 \\
\hline FOTL & TapeLift & 10 & 23 & 105 \\
\hline FOTL & TapeLift & 10 & 24 & 99 \\
\hline FOTL & TapeLift & 10 & 25 & 44 \\
\hline FOTL & TapeLift & 10 & 26 & 40 \\
\hline FOTL & TapeLift & 10 & 27 & 47 \\
\hline FOTL & TapeLift & 10 & 28 & 71 \\
\hline FOTL & TapeLift & 10 & 29 & 67 \\
\hline FOTL & TapeLift & 10 & 30 & 94 \\
\hline FOTL & TapeLift & 11 & 1 & 45 \\
\hline FOTL & TapeLift & 11 & 2 & 58 \\
\hline FOTL & TapeLift & 11 & 3 & 49 \\
\hline FOTL & TapeLift & 11 & 4 & 46 \\
\hline FOTL & TapeLift & 11 & 5 & 81 \\
\hline FOTL & TapeLift & 11 & 6 & 77 \\
\hline FOTL & TapeLift & 11 & 7 & 57 \\
\hline FOTL & TapeLift & 11 & 8 & 42 \\
\hline FOTL & TapeLift & 11 & 9 & 50 \\
\hline FOTL & TapeLift & 11 & 10 & 76 \\
\hline FOTL & TapeLift & 11 & 11 & 67 \\
\hline FOTL & TapeLift & 11 & 12 & 60 \\
\hline FOTL & TapeLift & 11 & 13 & 76 \\
\hline FOTL & TapeLift & 11 & 14 & 58 \\
\hline FOTL & TapeLift & 11 & 15 & 91 \\
\hline FOTL & TapeLift & 11 & 16 & 97 \\
\hline
\end{tabular}




\begin{tabular}{|c|c|c|c|c|}
\hline FOTL & TapeLift & 11 & 17 & 91 \\
\hline FOTL & TapeLift & 11 & 18 & 92 \\
\hline FOTL & TapeLift & 11 & 19 & 64 \\
\hline FOTL & TapeLift & 11 & 20 & 119 \\
\hline FOTL & TapeLift & 11 & 21 & 76 \\
\hline FOTL & TapeLift & 11 & 22 & 101 \\
\hline FOTL & TapeLift & 11 & 23 & 77 \\
\hline FOTL & TapeLift & 11 & 24 & 89 \\
\hline FOTL & TapeLift & 11 & 25 & 52 \\
\hline FOTL & TapeLift & 11 & 26 & 76 \\
\hline FOTL & TapeLift & 11 & 27 & 80 \\
\hline FOTL & TapeLift & 11 & 28 & 87 \\
\hline FOTL & TapeLift & 11 & 29 & 94 \\
\hline FOTL & TapeLift & 11 & 30 & 84 \\
\hline FOTL & TapeLift & 11 & 31 & 105 \\
\hline FOTL & TapeLift & 11 & 32 & 87 \\
\hline FOTL & TapeLift & 11 & 33 & 94 \\
\hline FOTL & TapeLift & 11 & 34 & 62 \\
\hline FOTL & TapeLift & 11 & 35 & 74 \\
\hline FOTL & TapeLift & 11 & 36 & 90 \\
\hline FOTL & TapeLift & 11 & 37 & 79 \\
\hline FOTL & TapeLift & 11 & 38 & 102 \\
\hline FOTL & TapeLift & 11 & 39 & 87 \\
\hline FOTL & TapeLift & 11 & 40 & 72 \\
\hline FOTL & TapeLift & 11 & 41 & 92 \\
\hline FOTL & TapeLift & 12 & 1 & 65 \\
\hline FOTL & TapeLift & 12 & 2 & 70 \\
\hline FOTL & TapeLift & 12 & 3 & 100 \\
\hline FOTL & TapeLift & 12 & 4 & 139 \\
\hline FOTL & TapeLift & 12 & 5 & 98 \\
\hline FOTL & TapeLift & 12 & 6 & 110 \\
\hline FOTL & TapeLift & 12 & 7 & 71 \\
\hline FOTL & TapeLift & 12 & 8 & 63 \\
\hline FOTL & TapeLift & 12 & 9 & 91 \\
\hline FOTL & TapeLift & 12 & 10 & 73 \\
\hline FOTL & TapeLift & 12 & 11 & 52 \\
\hline FOTL & TapeLift & 12 & 12 & 74 \\
\hline FOTL & TapeLift & 12 & 13 & 83 \\
\hline FOTL & TapeLift & 12 & 14 & 116 \\
\hline FOTL & TapeLift & 12 & 15 & 94 \\
\hline FOTL & TapeLift & 12 & 16 & 109 \\
\hline FOTL & TapeLift & 12 & 17 & 60 \\
\hline FOTL & TapeLift & 12 & 18 & 52 \\
\hline FOTL & TapeLift & 12 & 19 & 57 \\
\hline FOTL & TapeLift & 12 & 20 & 81 \\
\hline
\end{tabular}




\begin{tabular}{|c|c|c|c|c|}
\hline FOTL & TapeLift & 12 & 21 & 69 \\
\hline FOTL & TapeLift & 12 & 22 & 80 \\
\hline FOTL & TapeLift & 12 & 23 & 77 \\
\hline FOTL & TapeLift & 12 & 24 & 90 \\
\hline FOTL & TapeLift & 12 & 25 & 76 \\
\hline FOTL & TapeLift & 12 & 26 & 82 \\
\hline FOTL & TapeLift & 12 & 27 & 65 \\
\hline FOTL & TapeLift & 12 & 28 & 68 \\
\hline FOTL & TapeLift & 12 & 29 & 59 \\
\hline FOTL & TapeLift & 12 & 30 & 71 \\
\hline FOTL & TapeLift & 12 & 31 & 19 \\
\hline FOTL & TapeLift & 12 & 32 & 81 \\
\hline FOTL & TapeLift & 12 & 33 & 63 \\
\hline FOTL & TapeLift & 12 & 34 & 83 \\
\hline FOTL & TapeLift & 12 & 35 & 60 \\
\hline FOTL & TapeLift & 12 & 36 & 61 \\
\hline FOTL & TapeLift & 13 & 1 & 73 \\
\hline FOTL & TapeLift & 13 & 2 & 58 \\
\hline FOTL & TapeLift & 13 & 3 & 58 \\
\hline FOTL & TapeLift & 13 & 4 & 48 \\
\hline FOTL & TapeLift & 13 & 5 & 76 \\
\hline FOTL & TapeLift & 13 & 6 & 81 \\
\hline FOTL & TapeLift & 13 & 7 & 76 \\
\hline FOTL & TapeLift & 13 & 8 & 65 \\
\hline FOTL & TapeLift & 13 & 9 & 89 \\
\hline FOTL & TapeLift & 13 & 10 & 41 \\
\hline FOTL & TapeLift & 13 & 11 & 68 \\
\hline FOTL & TapeLift & 13 & 12 & 65 \\
\hline FOTL & TapeLift & 13 & 13 & 134 \\
\hline FOTL & TapeLift & 13 & 14 & 81 \\
\hline FOTL & TapeLift & 13 & 15 & 107 \\
\hline FOTL & TapeLift & 13 & 16 & 95 \\
\hline FOTL & TapeLift & 13 & 17 & 89 \\
\hline FOTL & TapeLift & 13 & 18 & 95 \\
\hline FOTL & TapeLift & 13 & 19 & 88 \\
\hline FOTL & TapeLift & 13 & 20 & 121 \\
\hline FOTL & TapeLift & 13 & 21 & 93 \\
\hline FOTL & TapeLift & 13 & 22 & 88 \\
\hline FOTL & TapeLift & 13 & 23 & 75 \\
\hline FOTL & TapeLift & 13 & 24 & 80 \\
\hline FOTL & TapeLift & 13 & 25 & 111 \\
\hline FOTL & TapeLift & 13 & 26 & 79 \\
\hline FOTL & TapeLift & 13 & 27 & 113 \\
\hline FOTL & TapeLift & 13 & 28 & 28 \\
\hline FOTL & TapeLift & 13 & 29 & 118 \\
\hline
\end{tabular}




\begin{tabular}{|c|c|c|c|c|}
\hline FOTL & TapeLift & 13 & 30 & 96 \\
\hline FOTL & TapeLift & 13 & 31 & 101 \\
\hline FOTL & TapeLift & 13 & 32 & 101 \\
\hline FOTL & TapeLift & 13 & 33 & 103 \\
\hline FOTL & TapeLift & 13 & 34 & 69 \\
\hline FOTL & TapeLift & 13 & 35 & 95 \\
\hline FOTL & TapeLift & 13 & 36 & 100 \\
\hline FOTL & TapeLift & 13 & 37 & 83 \\
\hline FOTL & TapeLift & 13 & 38 & 82 \\
\hline FOTL & TapeLift & 13 & 39 & 120 \\
\hline FOTL & TapeLift & 13 & 40 & 98 \\
\hline FOTL & TapeLift & 13 & 41 & 59 \\
\hline FOTL & TapeLift & 13 & 42 & 56 \\
\hline FOTL & TapeLift & 13 & 43 & 76 \\
\hline FOTL & TapeLift & 13 & 44 & 91 \\
\hline FOTL & TapeLift & 13 & 45 & 85 \\
\hline FOTL & TapeLift & 13 & 46 & 96 \\
\hline FOTL & TapeLift & 13 & 47 & 58 \\
\hline FOTL & TapeLift & 13 & 48 & 77 \\
\hline FOTL & TapeLift & 13 & 49 & 85 \\
\hline FOTL & TapeLift & 13 & 50 & 84 \\
\hline FOTL & TapeLift & 13 & 51 & 43 \\
\hline FOTL & TapeLift & 14 & 1 & 69 \\
\hline FOTL & TapeLift & 14 & 2 & 81 \\
\hline FOTL & TapeLift & 14 & 3 & 63 \\
\hline FOTL & TapeLift & 14 & 4 & 72 \\
\hline FOTL & TapeLift & 14 & 5 & 61 \\
\hline FOTL & TapeLift & 14 & 6 & 62 \\
\hline FOTL & TapeLift & 14 & 7 & 66 \\
\hline FOTL & TapeLift & 14 & 8 & 93 \\
\hline FOTL & TapeLift & 14 & 9 & 83 \\
\hline FOTL & TapeLift & 14 & 10 & 70 \\
\hline FOTL & TapeLift & 14 & 11 & 43 \\
\hline FOTL & TapeLift & 14 & 12 & 74 \\
\hline FOTL & TapeLift & 14 & 13 & 38 \\
\hline FOTL & TapeLift & 14 & 14 & 74 \\
\hline FOTL & TapeLift & 14 & 15 & 68 \\
\hline FOTL & TapeLift & 14 & 16 & 37 \\
\hline FOTL & TapeLift & 14 & 17 & 36 \\
\hline FOTL & TapeLift & 14 & 18 & 34 \\
\hline FOTL & TapeLift & 14 & 19 & 28 \\
\hline FOTL & TapeLift & 14 & 20 & 44 \\
\hline FOTL & TapeLift & 14 & 21 & 56 \\
\hline FOTL & TapeLift & 14 & 22 & 33 \\
\hline FOTL & TapeLift & 14 & 23 & 69 \\
\hline
\end{tabular}




\begin{tabular}{|c|c|c|c|c|}
\hline FOTL & TapeLift & 14 & 24 & 101 \\
\hline FOTL & TapeLift & 14 & 25 & 69 \\
\hline FOTL & TapeLift & 14 & 26 & 66 \\
\hline FOTL & TapeLift & 14 & 27 & 84 \\
\hline FOTL & TapeLift & 14 & 28 & 61 \\
\hline FOTL & TapeLift & 14 & 29 & 65 \\
\hline FOTL & TapeLift & 14 & 30 & 99 \\
\hline FOTL & TapeLift & 14 & 31 & 95 \\
\hline FOTL & TapeLift & 15 & 1 & 119 \\
\hline FOTL & TapeLift & 15 & 2 & 96 \\
\hline FOTL & TapeLift & 15 & 3 & 125 \\
\hline FOTL & TapeLift & 15 & 4 & 115 \\
\hline FOTL & TapeLift & 15 & 5 & 111 \\
\hline FOTL & TapeLift & 15 & 6 & 73 \\
\hline FOTL & TapeLift & 15 & 7 & 103 \\
\hline FOTL & TapeLift & 15 & 8 & 76 \\
\hline FOTL & TapeLift & 15 & 9 & 60 \\
\hline FOTL & TapeLift & 15 & 10 & 92 \\
\hline FOTL & TapeLift & 15 & 11 & 98 \\
\hline FOTL & TapeLift & 15 & 12 & 150 \\
\hline FOTL & TapeLift & 15 & 13 & 113 \\
\hline FOTL & TapeLift & 15 & 14 & 99 \\
\hline FOTL & TapeLift & 15 & 15 & 79 \\
\hline FOTL & TapeLift & 15 & 16 & 104 \\
\hline FOTL & TapeLift & 15 & 17 & 80 \\
\hline FOTL & TapeLift & 15 & 18 & 108 \\
\hline FOTL & TapeLift & 15 & 19 & 73 \\
\hline FOTL & TapeLift & 15 & 20 & 102 \\
\hline FOTL & TapeLift & 15 & 21 & 116 \\
\hline FOTL & TapeLift & 15 & 22 & 92 \\
\hline FOTL & TapeLift & 16 & 1 & 82 \\
\hline FOTL & TapeLift & 16 & 2 & 81 \\
\hline FOTL & TapeLift & 16 & 3 & 114 \\
\hline FOTL & TapeLift & 16 & 4 & 62 \\
\hline FOTL & TapeLift & 16 & 5 & 53 \\
\hline FOTL & TapeLift & 16 & 6 & 81 \\
\hline FOTL & TapeLift & 16 & 7 & 91 \\
\hline FOTL & TapeLift & 16 & 8 & 105 \\
\hline FOTL & TapeLift & 16 & 9 & 82 \\
\hline FOTL & TapeLift & 16 & 10 & 84 \\
\hline FOTL & TapeLift & 16 & 11 & 68 \\
\hline FOTL & TapeLift & 16 & 12 & 70 \\
\hline FOTL & TapeLift & 16 & 13 & 74 \\
\hline FOTL & TapeLift & 16 & 14 & 108 \\
\hline FOTL & TapeLift & 16 & 15 & 102 \\
\hline
\end{tabular}




\begin{tabular}{|c|c|c|c|c|}
\hline FOTL & TapeLift & 16 & 16 & 57 \\
\hline FOTL & TapeLift & 16 & 17 & 89 \\
\hline FOTL & TapeLift & 16 & 18 & 58 \\
\hline FOTL & TapeLift & 16 & 19 & 64 \\
\hline FOTL & TapeLift & 16 & 20 & 79 \\
\hline FOTL & TapeLift & 16 & 21 & 85 \\
\hline FOTL & TapeLift & 16 & 22 & 75 \\
\hline FOTL & TapeLift & 16 & 23 & 55 \\
\hline FOTL & TapeLift & 16 & 24 & 78 \\
\hline FOTL & TapeLift & 16 & 25 & 73 \\
\hline FOTL & TapeLift & 16 & 26 & 93 \\
\hline FOTL & TapeLift & 16 & 27 & 62 \\
\hline FOTL & TapeLift & 16 & 28 & 86 \\
\hline FOTL & TapeLift & 16 & 29 & 107 \\
\hline FOTL & TapeLift & 16 & 30 & 82 \\
\hline FOTL & TapeLift & 16 & 31 & 85 \\
\hline FOTL & TapeLift & 17 & 1 & 92 \\
\hline FOTL & TapeLift & 17 & 2 & 81 \\
\hline FOTL & TapeLift & 17 & 3 & 93 \\
\hline FOTL & TapeLift & 17 & 4 & 102 \\
\hline FOTL & TapeLift & 17 & 5 & 102 \\
\hline FOTL & TapeLift & 17 & 6 & 105 \\
\hline FOTL & TapeLift & 17 & 7 & 65 \\
\hline FOTL & TapeLift & 17 & 8 & 119 \\
\hline FOTL & TapeLift & 17 & 9 & 126 \\
\hline FOTL & TapeLift & 17 & 10 & 93 \\
\hline FOTL & TapeLift & 17 & 11 & 73 \\
\hline FOTL & TapeLift & 17 & 12 & 78 \\
\hline FOTL & TapeLift & 17 & 13 & 98 \\
\hline FOTL & TapeLift & 17 & 14 & 105 \\
\hline FOTL & TapeLift & 17 & 15 & 115 \\
\hline FOTL & TapeLift & 17 & 16 & 101 \\
\hline FOTL & TapeLift & 17 & 17 & 132 \\
\hline FOTL & TapeLift & 17 & 18 & 85 \\
\hline FOTL & TapeLift & 17 & 19 & 93 \\
\hline FOTL & TapeLift & 17 & 20 & 84 \\
\hline FOTL & TapeLift & 17 & 21 & 80 \\
\hline FOTL & TapeLift & 17 & 22 & 92 \\
\hline FOTL & TapeLift & 17 & 23 & 108 \\
\hline FOTL & TapeLift & 17 & 24 & 101 \\
\hline FOTL & TapeLift & 17 & 25 & 85 \\
\hline FOTL & TapeLift & 17 & 26 & 99 \\
\hline FOTL & TapeLift & 17 & 27 & 108 \\
\hline FOTL & TapeLift & 17 & 28 & 96 \\
\hline FOTL & TapeLift & 17 & 29 & 77 \\
\hline
\end{tabular}




\begin{tabular}{|c|c|c|c|c|}
\hline FOTL & TapeLift & 17 & 30 & 92 \\
\hline FOTL & TapeLift & 17 & 31 & 93 \\
\hline FOTL & TapeLift & 17 & 32 & 107 \\
\hline FOTL & TapeLift & 18 & 1 & 110 \\
\hline FOTL & TapeLift & 18 & 2 & 111 \\
\hline FOTL & TapeLift & 18 & 3 & 101 \\
\hline FOTL & TapeLift & 18 & 4 & 93 \\
\hline FOTL & TapeLift & 18 & 5 & 114 \\
\hline FOTL & TapeLift & 18 & 6 & 145 \\
\hline FOTL & TapeLift & 18 & 7 & 97 \\
\hline FOTL & TapeLift & 18 & 8 & 82 \\
\hline FOTL & TapeLift & 18 & 9 & 89 \\
\hline FOTL & TapeLift & 18 & 10 & 92 \\
\hline FOTL & TapeLift & 18 & 11 & 81 \\
\hline FOTL & TapeLift & 18 & 12 & 80 \\
\hline FOTL & TapeLift & 18 & 13 & 70 \\
\hline FOTL & TapeLift & 18 & 14 & 86 \\
\hline FOTL & TapeLift & 18 & 15 & 98 \\
\hline FOTL & TapeLift & 18 & 16 & 131 \\
\hline FOTL & TapeLift & 18 & 17 & 151 \\
\hline FOTL & TapeLift & 18 & 18 & 125 \\
\hline FOTL & TapeLift & 18 & 19 & 71 \\
\hline FOTL & TapeLift & 18 & 20 & 95 \\
\hline FOTL & TapeLift & 18 & 21 & 61 \\
\hline FOTL & TapeLift & 18 & 22 & 93 \\
\hline FOTL & TapeLift & 18 & 23 & 86 \\
\hline FOTL & TapeLift & 18 & 24 & 69 \\
\hline FOTL & TapeLift & 18 & 25 & 108 \\
\hline FOTL & TapeLift & 18 & 26 & 102 \\
\hline FOTL & TapeLift & 18 & 27 & 60 \\
\hline FOTL & TapeLift & 18 & 28 & 121 \\
\hline FOTL & TapeLift & 18 & 29 & 107 \\
\hline FOTL & TapeLift & 18 & 30 & 98 \\
\hline FOTL & TapeLift & 18 & 31 & 59 \\
\hline FOTL & TapeLift & 18 & 32 & 104 \\
\hline FOTL & TapeLift & 18 & 33 & 99 \\
\hline FOTL & TapeLift & 18 & 34 & 61 \\
\hline FOTL & TapeLift & 18 & 35 & 88 \\
\hline FOTL & TapeLift & 18 & 36 & 95 \\
\hline FOTL & TapeLift & 18 & 37 & 104 \\
\hline FOTL & TapeLift & 18 & 38 & 91 \\
\hline FOTL & TapeLift & 18 & 39 & 92 \\
\hline FOTL & TapeLift & 18 & 40 & 81 \\
\hline FOTL & TapeLift & 18 & 41 & 92 \\
\hline FOTL & TapeLift & 18 & 42 & 122 \\
\hline
\end{tabular}




\begin{tabular}{|c|c|c|c|c|}
\hline FOTL & TapeLift & 18 & 43 & 109 \\
\hline FOTL & TapeLift & 18 & 44 & 93 \\
\hline FOTL & TapeLift & 18 & 45 & 98 \\
\hline FOTL & TapeLift & 18 & 46 & 86 \\
\hline FOTL & TapeLift & 18 & 47 & 115 \\
\hline FOTL & TapeLift & 18 & 48 & 114 \\
\hline FOTL & TapeLift & 18 & 49 & 127 \\
\hline FOTL & TapeLift & 18 & 50 & 99 \\
\hline FOTL & TapeLift & 18 & 51 & 90 \\
\hline FOTL & TapeLift & 19 & 1 & 93 \\
\hline FOTL & TapeLift & 19 & 2 & 83 \\
\hline FOTL & TapeLift & 19 & 3 & 73 \\
\hline FOTL & TapeLift & 19 & 4 & 95 \\
\hline FOTL & TapeLift & 19 & 5 & 93 \\
\hline FOTL & TapeLift & 19 & 6 & 98 \\
\hline FOTL & TapeLift & 19 & 7 & 112 \\
\hline FOTL & TapeLift & 19 & 8 & 92 \\
\hline FOTL & TapeLift & 19 & 9 & 51 \\
\hline FOTL & TapeLift & 19 & 10 & 82 \\
\hline FOTL & TapeLift & 19 & 11 & 106 \\
\hline FOTL & TapeLift & 19 & 12 & 113 \\
\hline FOTL & TapeLift & 19 & 13 & 65 \\
\hline FOTL & TapeLift & 19 & 14 & 69 \\
\hline FOTL & TapeLift & 19 & 15 & 80 \\
\hline FOTL & TapeLift & 19 & 16 & 87 \\
\hline FOTL & TapeLift & 19 & 17 & 78 \\
\hline FOTL & TapeLift & 19 & 18 & 100 \\
\hline FOTL & TapeLift & 19 & 19 & 55 \\
\hline FOTL & TapeLift & 19 & 20 & 90 \\
\hline FOTL & TapeLift & 19 & 21 & 95 \\
\hline FOTL & TapeLift & 19 & 22 & 50 \\
\hline FOTL & TapeLift & 19 & 23 & 102 \\
\hline FOTL & TapeLift & 19 & 24 & 64 \\
\hline FOTL & TapeLift & 19 & 25 & 46 \\
\hline FOTL & TapeLift & 19 & 26 & 73 \\
\hline FOTL & TapeLift & 19 & 27 & 85 \\
\hline FOTL & TapeLift & 19 & 28 & 116 \\
\hline FOTL & TapeLift & 19 & 29 & 86 \\
\hline FOTL & TapeLift & 19 & 30 & 92 \\
\hline FOTL & TapeLift & 19 & 31 & 96 \\
\hline FOTL & TapeLift & 19 & 32 & 75 \\
\hline FOTL & TapeLift & 19 & 33 & 86 \\
\hline FOTL & TapeLift & 19 & 34 & 47 \\
\hline FOTL & TapeLift & 19 & 35 & 58 \\
\hline FOTL & TapeLift & 19 & 36 & 45 \\
\hline
\end{tabular}




\begin{tabular}{|c|c|c|c|c|}
\hline FOTL & TapeLift & 19 & 37 & 55 \\
\hline FOTL & TapeLift & 19 & 38 & 69 \\
\hline FOTL & TapeLift & 19 & 39 & 55 \\
\hline FOTL & TapeLift & 19 & 40 & 44 \\
\hline FOTL & TapeLift & 19 & 41 & 38 \\
\hline FOTL & TapeLift & 19 & 42 & 98 \\
\hline FOTL & TapeLift & 19 & 43 & 44 \\
\hline FOTL & TapeLift & 19 & 44 & 55 \\
\hline FOTL & TapeLift & 19 & 45 & 49 \\
\hline FOTL & TapeLift & 19 & 46 & 49 \\
\hline FOTL & TapeLift & 19 & 47 & 57 \\
\hline FOTL & TapeLift & 19 & 48 & 52 \\
\hline FOTL & TapeLift & 19 & 49 & 47 \\
\hline FOTL & TapeLift & 19 & 50 & 66 \\
\hline FOTL & TapeLift & 19 & 51 & 55 \\
\hline FOTL & TapeLift & 19 & 52 & 111 \\
\hline FOTL & TapeLift & 19 & 53 & 100 \\
\hline FOTL & TapeLift & 19 & 54 & 79 \\
\hline FOTL & TapeLift & 19 & 55 & 63 \\
\hline FOTL & TapeLift & 19 & 56 & 43 \\
\hline FOTL & TapeLift & 19 & 57 & 80 \\
\hline FOTL & TapeLift & 19 & 58 & 94 \\
\hline FOTL & TapeLift & 19 & 59 & 88 \\
\hline FOTL & TapeLift & 19 & 60 & 76 \\
\hline FOTL & TapeLift & 19 & 61 & 86 \\
\hline FOTL & TapeLift & 19 & 62 & 86 \\
\hline FOTL & TapeLift & 19 & 63 & 84 \\
\hline FOTL & TapeLift & 19 & 64 & 63 \\
\hline FOTL & TapeLift & 19 & 65 & 48 \\
\hline FOTL & TapeLift & 19 & 66 & 57 \\
\hline FOTL & TapeLift & 20 & 1 & 47 \\
\hline FOTL & TapeLift & 20 & 2 & 76 \\
\hline FOTL & TapeLift & 20 & 3 & 94 \\
\hline FOTL & TapeLift & 20 & 4 & 81 \\
\hline FOTL & TapeLift & 20 & 5 & 59 \\
\hline FOTL & TapeLift & 20 & 6 & 47 \\
\hline FOTL & TapeLift & 20 & 7 & 123 \\
\hline FOTL & TapeLift & 20 & 8 & 52 \\
\hline FOTL & TapeLift & 20 & 9 & 48 \\
\hline FOTL & TapeLift & 20 & 10 & 30 \\
\hline FOTL & TapeLift & 20 & 11 & 44 \\
\hline FOTL & TapeLift & 20 & 12 & 51 \\
\hline FOTL & TapeLift & 20 & 13 & 62 \\
\hline FOTL & TapeLift & 20 & 14 & 74 \\
\hline FOTL & TapeLift & 20 & 15 & 103 \\
\hline
\end{tabular}




\begin{tabular}{|c|c|c|c|c|}
\hline FOTL & TapeLift & 20 & 16 & 116 \\
\hline FOTL & TapeLift & 20 & 17 & 110 \\
\hline FOTL & TapeLift & 20 & 18 & 73 \\
\hline FOTL & TapeLift & 20 & 19 & 84 \\
\hline FOTL & TapeLift & 20 & 20 & 87 \\
\hline FOTL & TapeLift & 20 & 21 & 103 \\
\hline FOTL & TapeLift & 20 & 22 & 110 \\
\hline FOTL & TapeLift & 20 & 23 & 97 \\
\hline FOTL & TapeLift & 20 & 24 & 115 \\
\hline FOTL & TapeLift & 20 & 25 & 58 \\
\hline FOTL & TapeLift & 20 & 26 & 50 \\
\hline FOTL & TapeLift & 20 & 27 & 89 \\
\hline FOTL & TapeLift & 20 & 28 & 138 \\
\hline FOTL & TapeLift & 20 & 29 & 143 \\
\hline FOTL & TapeLift & 20 & 30 & 85 \\
\hline FOTL & TapeLift & 20 & 31 & 99 \\
\hline FOTL & TapeLift & 20 & 32 & 85 \\
\hline FOTL & TapeLift & 20 & 33 & 52 \\
\hline FOTL & TapeLift & 20 & 34 & 91 \\
\hline FOTL & TapeLift & 20 & 35 & 113 \\
\hline FOTL & TapeLift & 20 & 36 & 71 \\
\hline FOTL & TapeLift & 20 & 37 & 81 \\
\hline FOTL & TapeLift & 20 & 38 & 82 \\
\hline FOTL & TapeLift & 20 & 39 & 102 \\
\hline FOTL & TapeLift & 20 & 40 & 61 \\
\hline FOTL & TapeLift & 20 & 41 & 62 \\
\hline FOTL & TapeLift & 20 & 42 & 110 \\
\hline FOTL & TapeLift & 20 & 43 & 98 \\
\hline FOTL & TapeLift & 20 & 44 & 85 \\
\hline FOTL & TapeLift & 20 & 45 & 28 \\
\hline FOTL & TapeLift & 20 & 46 & 61 \\
\hline FOTL & TapeLift & 20 & 47 & 49 \\
\hline FOTL & TapeLift & 20 & 48 & 76 \\
\hline FOTL & TapeLift & 20 & 49 & 58 \\
\hline FOTL & TapeLift & 20 & 50 & 79 \\
\hline FOTL & TapeLift & 20 & 51 & 63 \\
\hline FOTL & TapeLift & 20 & 52 & 70 \\
\hline FOTL & TapeLift & 21 & 1 & 62 \\
\hline FOTL & TapeLift & 21 & 2 & 95 \\
\hline FOTL & TapeLift & 21 & 3 & 65 \\
\hline FOTL & TapeLift & 21 & 4 & 60 \\
\hline FOTL & TapeLift & 21 & 5 & 68 \\
\hline FOTL & TapeLift & 21 & 6 & 57 \\
\hline FOTL & TapeLift & 21 & 7 & 37 \\
\hline FOTL & TapeLift & 21 & 8 & 45 \\
\hline
\end{tabular}




\begin{tabular}{|c|c|c|c|}
\hline FOTL & TapeLift & 21 & 9 \\
\hline FOTL & TapeLift & 21 & 10 \\
\hline FOTL & TapeLift & 21 & 11 \\
\hline FOTL & TapeLift & 21 & 12 \\
\hline FOTL & TapeLift & 21 & 13 \\
\hline FOTL & TapeLift & 21 & 14 \\
\hline FOTL & TapeLift & 21 & 15 \\
\hline FOTL & TapeLift & 21 & 16 \\
\hline FOTL & TapeLift & 21 & 17 \\
\hline FOTL & TapeLift & 21 & 18 \\
\hline FOTL & TapeLift & 21 & 19 \\
\hline FOTL & TapeLift & 21 & 20 \\
\hline FOTL & TapeLift & 21 & 21 \\
\hline FOTL & TapeLift & 21 & 22 \\
\hline FOTL & TapeLift & 21 & 23 \\
\hline FOTL & TapeLift & 21 & 24 \\
\hline FOTL & TapeLift & 21 & 25 \\
\hline FOTL & TapeLift & 21 & 26 \\
\hline FOTL & TapeLift & 21 & 27 \\
\hline FOTL & TapeLift & 21 & 28 \\
\hline FOTL & TapeLift & 21 & 29 \\
\hline FOTL & TapeLift & 21 & 30 \\
\hline FOTL & TapeLift & 21 & 31 \\
\hline FOTL & TapeLift & 21 & 32 \\
\hline FOTL & TapeLift & 21 & 33 \\
\hline FOTL & TapeLift & 21 & 34 \\
\hline FOTL & TapeLift & 21 & 35 \\
\hline FOTL & TapeLift & 21 & 36 \\
\hline FOTL & TapeLift & 21 & 37 \\
\hline FOTL & TapeLift & 21 & 38 \\
\hline FOTL & TapeLift & 21 & 39 \\
\hline FOTL & TapeLift & 21 & 40 \\
\hline FOTL & TapeLift & 21 & 41 \\
\hline FOTL & TapeLift & 22 & 1 \\
\hline FOTL & TapeLift & 22 & 2 \\
\hline FOTL & TapeLift & 22 & 3 \\
\hline FOTL & TapeLift & 22 & 4 \\
\hline FOTL & TapeLift & 22 & 5 \\
\hline FOTL & TapeLift & 22 & 6 \\
\hline FOTL & TapeLift & 22 & 7 \\
\hline FOTL & TapeLift & 22 & 8 \\
\hline FOTL & TapeLift & 22 & 9 \\
\hline FOTL & TapeLift & 22 & 10 \\
\hline FOTL & TapeLift & 22 & 11 \\
\hline FOTL & TapeLift & 22 & 12 \\
\hline
\end{tabular}




\begin{tabular}{|c|c|c|c|c|}
\hline FOTL & TapeLift & 22 & 13 & 102 \\
\hline FOTL & TapeLift & 22 & 14 & 132 \\
\hline FOTL & TapeLift & 22 & 15 & 98 \\
\hline FOTL & TapeLift & 22 & 16 & 120 \\
\hline FOTL & TapeLift & 22 & 17 & 113 \\
\hline FOTL & TapeLift & 22 & 18 & 109 \\
\hline FOTL & TapeLift & 22 & 19 & 78 \\
\hline FOTL & TapeLift & 22 & 20 & 126 \\
\hline FOTL & TapeLift & 22 & 21 & 109 \\
\hline FOTL & TapeLift & 22 & 22 & 79 \\
\hline FOTL & TapeLift & 22 & 23 & 142 \\
\hline FOTL & TapeLift & 22 & 24 & 119 \\
\hline FOTL & TapeLift & 22 & 25 & 64 \\
\hline FOTL & TapeLift & 22 & 26 & 52 \\
\hline FOTL & TapeLift & 22 & 27 & 65 \\
\hline FOTL & TapeLift & 22 & 28 & 76 \\
\hline FOTL & TapeLift & 22 & 29 & 72 \\
\hline FOTL & TapeLift & 22 & 30 & 74 \\
\hline FOTL & TapeLift & 22 & 31 & 27 \\
\hline FOTL & TapeLift & 22 & 32 & 101 \\
\hline FOTL & TapeLift & 22 & 33 & 54 \\
\hline FOTL & TapeLift & 22 & 34 & 111 \\
\hline FOTL & TapeLift & 22 & 35 & 91 \\
\hline FOTL & TapeLift & 22 & 36 & 43 \\
\hline FOTL & TapeLift & 22 & 37 & 42 \\
\hline FOTL & TapeLift & 22 & 38 & 70 \\
\hline FOTL & TapeLift & 22 & 39 & 92 \\
\hline FOTL & TapeLift & 22 & 40 & 67 \\
\hline FOTL & TapeLift & 22 & 41 & 78 \\
\hline FOTL & TapeLift & 22 & 42 & 68 \\
\hline FOTL & TapeLift & 22 & 43 & 61 \\
\hline FOTL & TapeLift & 22 & 44 & 71 \\
\hline FOTL & TapeLift & 22 & 45 & 105 \\
\hline FOTL & TapeLift & 22 & 46 & 70 \\
\hline FOTL & TapeLift & 22 & 47 & 70 \\
\hline FOTL & TapeLift & 22 & 48 & 71 \\
\hline FOTL & TapeLift & 22 & 49 & 77 \\
\hline FOTL & TapeLift & 22 & 50 & 93 \\
\hline FOTL & TapeLift & 22 & 51 & 67 \\
\hline FOTL & TapeLift & 22 & 52 & 54 \\
\hline FOTL & TapeLift & 22 & 53 & 65 \\
\hline FOTL & TapeLift & 22 & 54 & 48 \\
\hline FOTL & TapeLift & 22 & 55 & 139 \\
\hline FOTL & TapeLift & 22 & 56 & 98 \\
\hline FOTL & TapeLift & 22 & 57 & 71 \\
\hline
\end{tabular}




\begin{tabular}{|c|c|c|c|c|}
\hline FOTL & TapeLift & 22 & 58 & 111 \\
\hline FOTL & TapeLift & 22 & 59 & 78 \\
\hline FOTL & TapeLift & 22 & 60 & 75 \\
\hline FOTL & TapeLift & 22 & 61 & 31 \\
\hline FOTL & TapeLift & 22 & 62 & 79 \\
\hline FOTL & TapeLift & 22 & 63 & 110 \\
\hline FOTL & TapeLift & 22 & 64 & 101 \\
\hline FOTL & TapeLift & 22 & 65 & 87 \\
\hline FOTL & TapeLift & 22 & 66 & 69 \\
\hline FOTL & TapeLift & 22 & 67 & 95 \\
\hline FOTL & TapeLift & 22 & 68 & 114 \\
\hline FOTL & TapeLift & 22 & 69 & 85 \\
\hline FOTL & TapeLift & 22 & 70 & 63 \\
\hline FOTL & TapeLift & 22 & 71 & 136 \\
\hline FOTL & TapeLift & 23 & 1 & 108 \\
\hline FOTL & TapeLift & 23 & 2 & 104 \\
\hline FOTL & TapeLift & 23 & 3 & 53 \\
\hline FOTL & TapeLift & 23 & 4 & 116 \\
\hline FOTL & TapeLift & 23 & 5 & 65 \\
\hline FOTL & TapeLift & 23 & 6 & 111 \\
\hline FOTL & TapeLift & 23 & 7 & 138 \\
\hline FOTL & TapeLift & 23 & 8 & 99 \\
\hline FOTL & TapeLift & 23 & 9 & 186 \\
\hline FOTL & TapeLift & 23 & 10 & 52 \\
\hline FOTL & TapeLift & 23 & 11 & 45 \\
\hline FOTL & TapeLift & 23 & 12 & 117 \\
\hline FOTL & TapeLift & 23 & 13 & 103 \\
\hline FOTL & TapeLift & 23 & 14 & 152 \\
\hline FOTL & TapeLift & 23 & 15 & 152 \\
\hline FOTL & TapeLift & 23 & 16 & 111 \\
\hline FOTL & TapeLift & 23 & 17 & 176 \\
\hline FOTL & TapeLift & 23 & 18 & 72 \\
\hline FOTL & TapeLift & 23 & 19 & 107 \\
\hline FOTL & TapeLift & 23 & 20 & 114 \\
\hline FOTL & TapeLift & 23 & 21 & 63 \\
\hline FOTL & TapeLift & 23 & 22 & 42 \\
\hline FOTL & TapeLift & 23 & 23 & 72 \\
\hline FOTL & TapeLift & 23 & 24 & 41 \\
\hline FOTL & TapeLift & 23 & 25 & 88 \\
\hline FOTL & TapeLift & 23 & 26 & 106 \\
\hline FOTL & TapeLift & 24 & 1 & 129 \\
\hline FOTL & TapeLift & 24 & 2 & 94 \\
\hline FOTL & TapeLift & 24 & 3 & 107 \\
\hline FOTL & TapeLift & 24 & 4 & 104 \\
\hline FOTL & TapeLift & 24 & 5 & 91 \\
\hline
\end{tabular}




\begin{tabular}{|c|c|c|c|c|}
\hline FOTL & TapeLift & 24 & 6 & 81 \\
\hline FOTL & TapeLift & 24 & 7 & 95 \\
\hline FOTL & TapeLift & 24 & 8 & 83 \\
\hline FOTL & TapeLift & 24 & 9 & 66 \\
\hline FOTL & TapeLift & 24 & 10 & 69 \\
\hline FOTL & TapeLift & 24 & 11 & 106 \\
\hline FOTL & TapeLift & 24 & 12 & 93 \\
\hline FOTL & TapeLift & 24 & 13 & 96 \\
\hline FOTL & TapeLift & 24 & 14 & 88 \\
\hline FOTL & TapeLift & 24 & 15 & 108 \\
\hline FOTL & TapeLift & 24 & 16 & 80 \\
\hline FOTL & TapeLift & 24 & 17 & 82 \\
\hline FOTL & TapeLift & 24 & 18 & 51 \\
\hline FOTL & TapeLift & 24 & 19 & 56 \\
\hline FOTL & TapeLift & 24 & 20 & 57 \\
\hline FOTL & TapeLift & 24 & 21 & 84 \\
\hline FOTL & TapeLift & 24 & 22 & 67 \\
\hline FOTL & TapeLift & 24 & 23 & 63 \\
\hline FOTL & TapeLift & 24 & 24 & 111 \\
\hline FOTL & TapeLift & 24 & 25 & 92 \\
\hline FOTL & TapeLift & 24 & 26 & 103 \\
\hline FOTL & TapeLift & 24 & 27 & 87 \\
\hline FOTL & TapeLift & 24 & 28 & 63 \\
\hline FOTL & TapeLift & 24 & 29 & 57 \\
\hline FOTL & TapeLift & 24 & 30 & 74 \\
\hline FOTL & TapeLift & 24 & 31 & 92 \\
\hline FOTL & TapeLift & 24 & 32 & 88 \\
\hline FOTL & TapeLift & 24 & 33 & 99 \\
\hline FOTL & TapeLift & 24 & 34 & 103 \\
\hline FOTL & TapeLift & 24 & 35 & 73 \\
\hline FOTL & TapeLift & 25 & 1 & 119 \\
\hline FOTL & TapeLift & 25 & 2 & 95 \\
\hline FOTL & TapeLift & 25 & 3 & 78 \\
\hline FOTL & TapeLift & 25 & 4 & 75 \\
\hline FOTL & TapeLift & 25 & 5 & 89 \\
\hline FOTL & TapeLift & 25 & 6 & 97 \\
\hline FOTL & TapeLift & 25 & 7 & 43 \\
\hline FOTL & TapeLift & 25 & 8 & 70 \\
\hline FOTL & TapeLift & 25 & 9 & 75 \\
\hline FOTL & TapeLift & 25 & 10 & 102 \\
\hline FOTL & TapeLift & 25 & 11 & 96 \\
\hline FOTL & TapeLift & 25 & 12 & 76 \\
\hline FOTL & TapeLift & 25 & 13 & 128 \\
\hline FOTL & TapeLift & 25 & 14 & 74 \\
\hline FOTL & TapeLift & 25 & 15 & 77 \\
\hline
\end{tabular}




\begin{tabular}{|c|c|c|c|c|}
\hline FOTL & TapeLift & 25 & 16 & 116 \\
\hline FOTL & TapeLift & 25 & 17 & 104 \\
\hline FOTL & TapeLift & 25 & 18 & 88 \\
\hline FOTL & TapeLift & 25 & 19 & 87 \\
\hline FOTL & TapeLift & 25 & 20 & 103 \\
\hline FOTL & TapeLift & 25 & 21 & 122 \\
\hline FOTL & TapeLift & 25 & 22 & 98 \\
\hline FOTL & TapeLift & 25 & 23 & 112 \\
\hline FOTL & TapeLift & 25 & 24 & 34 \\
\hline FOTL & TapeLift & 25 & 25 & 109 \\
\hline FOTL & TapeLift & 25 & 26 & 77 \\
\hline FOTL & TapeLift & 25 & 27 & 68 \\
\hline FOTL & TapeLift & 25 & 28 & 73 \\
\hline FOTL & TapeLift & 25 & 29 & 85 \\
\hline FOTL & TapeLift & 25 & 30 & 155 \\
\hline FOTL & TapeLift & 25 & 31 & 58 \\
\hline FOTL & TapeLift & 25 & 32 & 95 \\
\hline FOTL & TapeLift & 25 & 33 & 104 \\
\hline FOTL & TapeLift & 25 & 34 & 69 \\
\hline FOTL & TapeLift & 25 & 35 & 36 \\
\hline FOTL & TapeLift & 25 & 36 & 17 \\
\hline FOTL & TapeLift & 25 & 37 & 32 \\
\hline FOTL & TapeLift & 25 & 38 & 43 \\
\hline FOTL & TapeLift & 25 & 39 & 63 \\
\hline FOTL & TapeLift & 25 & 40 & 40 \\
\hline FOTL & TapeLift & 25 & 41 & 43 \\
\hline FOTL & TapeLift & 25 & 42 & 54 \\
\hline FOTL & TapeLift & 25 & 43 & 65 \\
\hline FOTL & TapeLift & 25 & 44 & 70 \\
\hline FOTL & TapeLift & 25 & 45 & 66 \\
\hline FOTL & TapeLift & 25 & 46 & 76 \\
\hline FOTL & TapeLift & 26 & 1 & 82 \\
\hline FOTL & TapeLift & 26 & 2 & 72 \\
\hline FOTL & TapeLift & 26 & 3 & 64 \\
\hline FOTL & TapeLift & 26 & 4 & 63 \\
\hline FOTL & TapeLift & 26 & 5 & 74 \\
\hline FOTL & TapeLift & 26 & 6 & 63 \\
\hline FOTL & TapeLift & 26 & 7 & 61 \\
\hline FOTL & TapeLift & 26 & 8 & 45 \\
\hline FOTL & TapeLift & 26 & 9 & 47 \\
\hline FOTL & TapeLift & 26 & 10 & 67 \\
\hline FOTL & TapeLift & 26 & 11 & 44 \\
\hline FOTL & TapeLift & 26 & 12 & 42 \\
\hline FOTL & TapeLift & 26 & 13 & 68 \\
\hline FOTL & TapeLift & 26 & 14 & 58 \\
\hline
\end{tabular}




\begin{tabular}{|c|c|c|c|c|}
\hline FOTL & TapeLift & 26 & 15 & 61 \\
\hline FOTL & TapeLift & 26 & 16 & 37 \\
\hline FOTL & TapeLift & 26 & 17 & 45 \\
\hline FOTL & TapeLift & 26 & 18 & 28 \\
\hline FOTL & TapeLift & 26 & 19 & 47 \\
\hline FOTL & TapeLift & 26 & 20 & 67 \\
\hline FOTL & TapeLift & 26 & 21 & 55 \\
\hline FOTL & TapeLift & 26 & 22 & 75 \\
\hline FOTL & TapeLift & 26 & 23 & 61 \\
\hline FOTL & TapeLift & 26 & 24 & 80 \\
\hline FOTL & TapeLift & 26 & 25 & 67 \\
\hline FOTL & TapeLift & 26 & 26 & 73 \\
\hline FOTL & TapeLift & 26 & 27 & 68 \\
\hline FOTL & TapeLift & 26 & 28 & 87 \\
\hline FOTL & TapeLift & 26 & 29 & 97 \\
\hline FOTL & TapeLift & 26 & 30 & 67 \\
\hline FOTL & TapeLift & 26 & 31 & 55 \\
\hline FOTL & TapeLift & 26 & 32 & 60 \\
\hline FOTL & TapeLift & 26 & 33 & 81 \\
\hline FOTL & TapeLift & 26 & 34 & 37 \\
\hline FOTL & TapeLift & 26 & 35 & 51 \\
\hline FOTL & TapeLift & 26 & 36 & 59 \\
\hline FOTL & TapeLift & 26 & 37 & 79 \\
\hline FOTL & TapeLift & 26 & 38 & 56 \\
\hline FOTL & TapeLift & 26 & 39 & 52 \\
\hline FOTL & TapeLift & 26 & 40 & 61 \\
\hline FOTL & TapeLift & 26 & 41 & 60 \\
\hline FOTL & TapeLift & 26 & 42 & 55 \\
\hline FOTL & TapeLift & 26 & 43 & 95 \\
\hline FOTL & TapeLift & 27 & 1 & 49 \\
\hline FOTL & TapeLift & 27 & 2 & 67 \\
\hline FOTL & TapeLift & 27 & 3 & 78 \\
\hline FOTL & TapeLift & 27 & 4 & 45 \\
\hline FOTL & TapeLift & 27 & 5 & 70 \\
\hline FOTL & TapeLift & 27 & 6 & 72 \\
\hline FOTL & TapeLift & 27 & 7 & 50 \\
\hline FOTL & TapeLift & 27 & 8 & 65 \\
\hline FOTL & TapeLift & 27 & 9 & 119 \\
\hline FOTL & TapeLift & 27 & 10 & 56 \\
\hline FOTL & TapeLift & 27 & 11 & 67 \\
\hline FOTL & TapeLift & 27 & 12 & 88 \\
\hline FOTL & TapeLift & 27 & 13 & 117 \\
\hline FOTL & TapeLift & 27 & 14 & 78 \\
\hline FOTL & TapeLift & 27 & 15 & 58 \\
\hline FOTL & TapeLift & 27 & 16 & 63 \\
\hline
\end{tabular}




\begin{tabular}{|c|c|c|c|c|}
\hline FOTL & TapeLift & 27 & 17 & 80 \\
\hline FOTL & TapeLift & 27 & 18 & 65 \\
\hline FOTL & TapeLift & 27 & 19 & 69 \\
\hline FOTL & TapeLift & 27 & 20 & 88 \\
\hline FOTL & TapeLift & 27 & 21 & 86 \\
\hline FOTL & TapeLift & 27 & 22 & 72 \\
\hline FOTL & TapeLift & 27 & 23 & 57 \\
\hline FOTL & TapeLift & 27 & 24 & 66 \\
\hline FOTL & TapeLift & 27 & 25 & 58 \\
\hline FOTL & TapeLift & 27 & 26 & 70 \\
\hline FOTL & TapeLift & 27 & 27 & 151 \\
\hline FOTL & TapeLift & 27 & 28 & 42 \\
\hline FOTL & TapeLift & 27 & 29 & 58 \\
\hline FOTL & TapeLift & 27 & 30 & 56 \\
\hline FOTL & TapeLift & 27 & 31 & 104 \\
\hline FOTL & TapeLift & 27 & 32 & 103 \\
\hline FOTL & TapeLift & 27 & 33 & 101 \\
\hline FOTL & TapeLift & 27 & 34 & 118 \\
\hline FOTL & TapeLift & 27 & 35 & 141 \\
\hline FOTL & TapeLift & 27 & 36 & 126 \\
\hline FOTL & TapeLift & 28 & 1 & 120 \\
\hline FOTL & TapeLift & 28 & 2 & 89 \\
\hline FOTL & TapeLift & 28 & 3 & 102 \\
\hline FOTL & TapeLift & 28 & 4 & 106 \\
\hline FOTL & TapeLift & 28 & 5 & 119 \\
\hline FOTL & TapeLift & 28 & 6 & 79 \\
\hline FOTL & TapeLift & 28 & 7 & 97 \\
\hline FOTL & TapeLift & 28 & 8 & 77 \\
\hline FOTL & TapeLift & 28 & 9 & 105 \\
\hline FOTL & TapeLift & 28 & 10 & 88 \\
\hline FOTL & TapeLift & 28 & 11 & 73 \\
\hline FOTL & TapeLift & 28 & 12 & 116 \\
\hline FOTL & TapeLift & 28 & 13 & 85 \\
\hline FOTL & TapeLift & 28 & 14 & 114 \\
\hline FOTL & TapeLift & 28 & 15 & 100 \\
\hline FOTL & TapeLift & 28 & 16 & 128 \\
\hline FOTL & TapeLift & 28 & 17 & 133 \\
\hline FOTL & TapeLift & 28 & 18 & 87 \\
\hline FOTL & TapeLift & 28 & 19 & 114 \\
\hline FOTL & TapeLift & 28 & 20 & 97 \\
\hline FOTL & TapeLift & 28 & 21 & 90 \\
\hline FOTL & TapeLift & 28 & 22 & 82 \\
\hline FOTL & TapeLift & 28 & 23 & 103 \\
\hline FOTL & TapeLift & 28 & 24 & 66 \\
\hline FOTL & TapeLift & 28 & 25 & 107 \\
\hline
\end{tabular}




\begin{tabular}{|c|c|c|c|c|}
\hline FOTL & TapeLift & 28 & 26 & 77 \\
\hline FOTL & TapeLift & 28 & 27 & 72 \\
\hline FOTL & TapeLift & 28 & 28 & 90 \\
\hline FOTL & TapeLift & 28 & 29 & 55 \\
\hline FOTL & TapeLift & 28 & 30 & 43 \\
\hline FOTL & TapeLift & 28 & 31 & 80 \\
\hline FOTL & TapeLift & 28 & 32 & 93 \\
\hline FOTL & TapeLift & 28 & 33 & 112 \\
\hline FOTL & TapeLift & 28 & 34 & 125 \\
\hline FOTL & TapeLift & 28 & 35 & 99 \\
\hline FOTL & TapeLift & 28 & 36 & 58 \\
\hline FOTL & TapeLift & 28 & 37 & 74 \\
\hline FOTL & TapeLift & 28 & 38 & 64 \\
\hline FOTL & TapeLift & 28 & 39 & 83 \\
\hline FOTL & TapeLift & 28 & 40 & 104 \\
\hline FOTL & TapeLift & 28 & 41 & 54 \\
\hline FOTL & TapeLift & 28 & 42 & 86 \\
\hline FOTL & TapeLift & 28 & 43 & 86 \\
\hline FOTL & TapeLift & 29 & 1 & 79 \\
\hline FOTL & TapeLift & 29 & 2 & 89 \\
\hline FOTL & TapeLift & 29 & 3 & 61 \\
\hline FOTL & TapeLift & 29 & 4 & 87 \\
\hline FOTL & TapeLift & 29 & 5 & 65 \\
\hline FOTL & TapeLift & 29 & 6 & 73 \\
\hline FOTL & TapeLift & 29 & 7 & 40 \\
\hline FOTL & TapeLift & 29 & 8 & 67 \\
\hline FOTL & TapeLift & 29 & 9 & 48 \\
\hline FOTL & TapeLift & 29 & 10 & 62 \\
\hline FOTL & TapeLift & 29 & 11 & 117 \\
\hline FOTL & TapeLift & 29 & 12 & 93 \\
\hline FOTL & TapeLift & 29 & 13 & 84 \\
\hline FOTL & TapeLift & 29 & 14 & 106 \\
\hline FOTL & TapeLift & 29 & 15 & 72 \\
\hline FOTL & TapeLift & 29 & 16 & 118 \\
\hline FOTL & TapeLift & 29 & 17 & 78 \\
\hline FOTL & TapeLift & 29 & 18 & 58 \\
\hline FOTL & TapeLift & 29 & 19 & 115 \\
\hline FOTL & TapeLift & 29 & 20 & 102 \\
\hline FOTL & TapeLift & 29 & 21 & 61 \\
\hline FOTL & TapeLift & 29 & 22 & 68 \\
\hline FOTL & TapeLift & 29 & 23 & 87 \\
\hline FOTL & TapeLift & 29 & 24 & 98 \\
\hline FOTL & TapeLift & 29 & 25 & 30 \\
\hline FOTL & TapeLift & 29 & 26 & 91 \\
\hline FOTL & TapeLift & 29 & 27 & 92 \\
\hline
\end{tabular}




\begin{tabular}{|c|c|c|c|c|}
\hline FOTL & TapeLift & 30 & 1 & 80 \\
\hline FOTL & TapeLift & 30 & 2 & 105 \\
\hline FOTL & TapeLift & 30 & 3 & 136 \\
\hline FOTL & TapeLift & 30 & 4 & 115 \\
\hline FOTL & TapeLift & 30 & 5 & 69 \\
\hline FOTL & TapeLift & 30 & 6 & 85 \\
\hline FOTL & TapeLift & 30 & 7 & 86 \\
\hline FOTL & TapeLift & 30 & 8 & 84 \\
\hline FOTL & TapeLift & 30 & 9 & 99 \\
\hline FOTL & TapeLift & 30 & 10 & 90 \\
\hline FOTL & TapeLift & 30 & 11 & 104 \\
\hline FOTL & TapeLift & 30 & 12 & 71 \\
\hline FOTL & TapeLift & 30 & 13 & 97 \\
\hline FOTL & TapeLift & 30 & 14 & 94 \\
\hline FOTL & TapeLift & 30 & 15 & 119 \\
\hline FOTL & TapeLift & 30 & 16 & 113 \\
\hline FOTL & TapeLift & 30 & 17 & 97 \\
\hline FOTL & TapeLift & 30 & 18 & 111 \\
\hline FOTL & TapeLift & 30 & 19 & 84 \\
\hline FOTL & TapeLift & 30 & 20 & 106 \\
\hline FOTL & TapeLift & 30 & 21 & 113 \\
\hline FOTL & TapeLift & 30 & 22 & 99 \\
\hline FOTL & TapeLift & 30 & 23 & 77 \\
\hline FOTL & TapeLift & 30 & 24 & 68 \\
\hline FOTL & TapeLift & 30 & 25 & 98 \\
\hline FOTL & TapeLift & 30 & 26 & 66 \\
\hline FOTL & TapeLift & 30 & 27 & 112 \\
\hline FOTL & TapeLift & 30 & 28 & 68 \\
\hline FOTL & TapeLift & 30 & 29 & 84 \\
\hline FOTL & TapeLift & 30 & 30 & 102 \\
\hline FOTL & TapeLift & 31 & 1 & 100 \\
\hline FOTL & TapeLift & 31 & 2 & 93 \\
\hline FOTL & TapeLift & 31 & 3 & 101 \\
\hline FOTL & TapeLift & 31 & 4 & 138 \\
\hline FOTL & TapeLift & 31 & 5 & 112 \\
\hline FOTL & TapeLift & 31 & 6 & 101 \\
\hline FOTL & TapeLift & 31 & 7 & 92 \\
\hline FOTL & TapeLift & 31 & 8 & 105 \\
\hline FOTL & TapeLift & 31 & 9 & 76 \\
\hline FOTL & TapeLift & 31 & 10 & 113 \\
\hline FOTL & TapeLift & 31 & 11 & 108 \\
\hline FOTL & TapeLift & 31 & 12 & 60 \\
\hline FOTL & TapeLift & 31 & 13 & 48 \\
\hline FOTL & TapeLift & 31 & 14 & 122 \\
\hline FOTL & TapeLift & 31 & 15 & 103 \\
\hline
\end{tabular}




\begin{tabular}{|c|c|c|c|c|}
\hline FOTL & TapeLift & 32 & 1 & 84 \\
\hline FOTL & TapeLift & 32 & 2 & 93 \\
\hline FOTL & TapeLift & 32 & 3 & 81 \\
\hline FOTL & TapeLift & 32 & 4 & 75 \\
\hline FOTL & TapeLift & 32 & 5 & 69 \\
\hline FOTL & TapeLift & 32 & 6 & 104 \\
\hline FOTL & TapeLift & 32 & 7 & 101 \\
\hline FOTL & TapeLift & 32 & 8 & 99 \\
\hline FOTL & TapeLift & 32 & 9 & 43 \\
\hline FOTL & TapeLift & 32 & 10 & 80 \\
\hline FOTL & TapeLift & 32 & 11 & 63 \\
\hline FOTL & TapeLift & 32 & 12 & 74 \\
\hline FOTL & TapeLift & 32 & 13 & 70 \\
\hline FOTL & TapeLift & 32 & 14 & 88 \\
\hline FOTL & TapeLift & 32 & 15 & 83 \\
\hline FOTL & TapeLift & 32 & 16 & 124 \\
\hline FOTL & TapeLift & 32 & 17 & 114 \\
\hline FOTL & TapeLift & 32 & 18 & 142 \\
\hline FOTL & TapeLift & 32 & 19 & 132 \\
\hline FOTL & TapeLift & 32 & 20 & 50 \\
\hline FOTL & TapeLift & 32 & 21 & 120 \\
\hline FOTL & TapeLift & 32 & 22 & 85 \\
\hline FOTL & TapeLift & 32 & 23 & 73 \\
\hline FOTL & TapeLift & 32 & 24 & 65 \\
\hline FOTL & TapeLift & 32 & 25 & 73 \\
\hline FOTL & TapeLift & 32 & 26 & 70 \\
\hline FOTL & TapeLift & 32 & 27 & 80 \\
\hline FOTL & TapeLift & 33 & 1 & 72 \\
\hline FOTL & TapeLift & 33 & 2 & 97 \\
\hline FOTL & TapeLift & 33 & 3 & 108 \\
\hline FOTL & TapeLift & 33 & 4 & 54 \\
\hline FOTL & TapeLift & 33 & 5 & 84 \\
\hline FOTL & TapeLift & 33 & 6 & 85 \\
\hline FOTL & TapeLift & 33 & 7 & 79 \\
\hline FOTL & TapeLift & 33 & 8 & 65 \\
\hline FOTL & TapeLift & 33 & 9 & 87 \\
\hline FOTL & TapeLift & 33 & 10 & 74 \\
\hline FOTL & TapeLift & 33 & 11 & 78 \\
\hline FOTL & TapeLift & 33 & 12 & 63 \\
\hline FOTL & TapeLift & 33 & 13 & 92 \\
\hline FOTL & TapeLift & 33 & 14 & 118 \\
\hline FOTL & TapeLift & 33 & 15 & 75 \\
\hline FOTL & TapeLift & 33 & 16 & 59 \\
\hline FOTL & TapeLift & 33 & 17 & 89 \\
\hline FOTL & TapeLift & 33 & 18 & 89 \\
\hline
\end{tabular}




\begin{tabular}{|c|c|c|c|c|}
\hline FOTL & TapeLift & 33 & 19 & 108 \\
\hline FOTL & TapeLift & 33 & 20 & 102 \\
\hline FOTL & TapeLift & 33 & 21 & 92 \\
\hline FOTL & TapeLift & 34 & 1 & 80 \\
\hline FOTL & TapeLift & 34 & 2 & 81 \\
\hline FOTL & TapeLift & 34 & 3 & 67 \\
\hline FOTL & TapeLift & 34 & 4 & 81 \\
\hline FOTL & TapeLift & 34 & 5 & 89 \\
\hline FOTL & TapeLift & 34 & 6 & 88 \\
\hline FOTL & TapeLift & 34 & 7 & 107 \\
\hline FOTL & TapeLift & 34 & 8 & 89 \\
\hline FOTL & TapeLift & 34 & 9 & 100 \\
\hline FOTL & TapeLift & 34 & 10 & 72 \\
\hline FOTL & TapeLift & 34 & 11 & 50 \\
\hline FOTL & TapeLift & 34 & 12 & 70 \\
\hline FOTL & TapeLift & 34 & 13 & 53 \\
\hline FOTL & TapeLift & 34 & 14 & 73 \\
\hline FOTL & TapeLift & 34 & 15 & 96 \\
\hline FOTL & TapeLift & 34 & 16 & 101 \\
\hline FOTL & TapeLift & 34 & 17 & 49 \\
\hline FOTL & TapeLift & 34 & 18 & 74 \\
\hline FOTL & TapeLift & 34 & 19 & 70 \\
\hline FOTL & TapeLift & 34 & 20 & 64 \\
\hline FOTL & TapeLift & 34 & 21 & 56 \\
\hline FOTL & TapeLift & 34 & 22 & 52 \\
\hline FOTL & TapeLift & 35 & 1 & 66 \\
\hline FOTL & TapeLift & 35 & 2 & 82 \\
\hline FOTL & TapeLift & 35 & 3 & 71 \\
\hline FOTL & TapeLift & 35 & 4 & 49 \\
\hline FOTL & TapeLift & 35 & 5 & 72 \\
\hline FOTL & TapeLift & 35 & 6 & 99 \\
\hline FOTL & TapeLift & 35 & 7 & 113 \\
\hline FOTL & TapeLift & 35 & 8 & 101 \\
\hline FOTL & TapeLift & 35 & 9 & 109 \\
\hline FOTL & TapeLift & 35 & 10 & 75 \\
\hline FOTL & Vacuum & 2 & 1 & 49 \\
\hline FOTL & Vacuum & 2 & 2 & 105 \\
\hline FOTL & Vacuum & 2 & 3 & 35 \\
\hline FOTL & Vacuum & 2 & 4 & 17 \\
\hline FOTL & Vacuum & 2 & 5 & 42 \\
\hline FOTL & Vacuum & 2 & 6 & 109 \\
\hline FOTL & Vacuum & 2 & 7 & 22 \\
\hline FOTL & Vacuum & 2 & 8 & 46 \\
\hline FOTL & Vacuum & 2 & 9 & 45 \\
\hline FOTL & Vacuum & 2 & 10 & 113 \\
\hline
\end{tabular}




\begin{tabular}{|c|c|c|c|}
\hline FOTL & Vacuum & 2 & 11 \\
\hline FOTL & Vacuum & 2 & 12 \\
\hline FOTL & Vacuum & 3 & 1 \\
\hline FOTL & Vacuum & 3 & 2 \\
\hline FOTL & Vacuum & 3 & 3 \\
\hline FOTL & Vacuum & 3 & 4 \\
\hline FOTL & Vacuum & 3 & 5 \\
\hline FOTL & Vacuum & 3 & 6 \\
\hline FOTL & Vacuum & 3 & 7 \\
\hline FOTL & Vacuum & 3 & 8 \\
\hline FOTL & Vacuum & 3 & 9 \\
\hline FOTL & Vacuum & 3 & 10 \\
\hline FOTL & Vacuum & 3 & 11 \\
\hline FOTL & Vacuum & 3 & 12 \\
\hline FOTL & Vacuum & 4 & 1 \\
\hline FOTL & Vacuum & 4 & 2 \\
\hline FOTL & Vacuum & 4 & 3 \\
\hline FOTL & Vacuum & 4 & 4 \\
\hline FOTL & Vacuum & 4 & 5 \\
\hline FOTL & Vacuum & 4 & 6 \\
\hline FOTL & Vacuum & 4 & 7 \\
\hline FOTL & Vacuum & 4 & 8 \\
\hline FOTL & Vacuum & 4 & 9 \\
\hline FOTL & Vacuum & 4 & 10 \\
\hline FOTL & Vacuum & 4 & 11 \\
\hline FOTL & Vacuum & 4 & 12 \\
\hline FOTL & Vacuum & 4 & 13 \\
\hline FOTL & Vacuum & 4 & 14 \\
\hline FOTL & Vacuum & 5 & 1 \\
\hline FOTL & Vacuum & 5 & 2 \\
\hline FOTL & Vacuum & 5 & 3 \\
\hline FOTL & Vacuum & 5 & 4 \\
\hline FOTL & Vacuum & 5 & 5 \\
\hline FOTL & Vacuum & 5 & 6 \\
\hline FOTL & Vacuum & 5 & 7 \\
\hline FOTL & Vacuum & 5 & 8 \\
\hline FOTL & Vacuum & 5 & 9 \\
\hline FOTL & Vacuum & 5 & 10 \\
\hline FOTL & Vacuum & 5 & 11 \\
\hline FOTL & Vacuum & 5 & 12 \\
\hline FOTL & Vacuum & 5 & 13 \\
\hline FOTL & Vacuum & 5 & 14 \\
\hline FOTL & Vacuum & 5 & 15 \\
\hline FOTL & Vacuum & 6 & 1 \\
\hline FOTL & Vacuum & 6 & 2 \\
\hline
\end{tabular}




\begin{tabular}{|c|c|c|c|}
\hline FOTL & Vacuum & 6 & 3 \\
\hline FOTL & Vacuum & 6 & 4 \\
\hline FOTL & Vacuum & 6 & 5 \\
\hline FOTL & Vacuum & 6 & 6 \\
\hline FOTL & Vacuum & 6 & 7 \\
\hline FOTL & Vacuum & 6 & 8 \\
\hline FOTL & Vacuum & 6 & 9 \\
\hline FOTL & Vacuum & 6 & 10 \\
\hline FOTL & Vacuum & 7 & 1 \\
\hline FOTL & Vacuum & 7 & 2 \\
\hline FOTL & Vacuum & 7 & 3 \\
\hline FOTL & Vacuum & 7 & 4 \\
\hline FOTL & Vacuum & 7 & 5 \\
\hline FOTL & Vacuum & 7 & 6 \\
\hline FOTL & Vacuum & 7 & 7 \\
\hline FOTL & Vacuum & 7 & 8 \\
\hline FOTL & Vacuum & 7 & 9 \\
\hline FOTL & Vacuum & 7 & 10 \\
\hline FOTL & Vacuum & 8 & 1 \\
\hline FOTL & Vacuum & 8 & 2 \\
\hline FOTL & Vacuum & 8 & 3 \\
\hline FOTL & Vacuum & 8 & 4 \\
\hline FOTL & Vacuum & 8 & 5 \\
\hline FOTL & Vacuum & 8 & 6 \\
\hline FOTL & Vacuum & 8 & 7 \\
\hline FOTL & Vacuum & 8 & 8 \\
\hline FOTL & Vacuum & 8 & 9 \\
\hline FOTL & Vacuum & 8 & 10 \\
\hline FOTL & Vacuum & 8 & 11 \\
\hline FOTL & Vacuum & 8 & 12 \\
\hline FOTL & Vacuum & 8 & 13 \\
\hline FOTL & Vacuum & 8 & 14 \\
\hline FOTL & Vacuum & 8 & 15 \\
\hline FOTL & Vacuum & 8 & 16 \\
\hline FOTL & Vacuum & 8 & 17 \\
\hline FOTL & Vacuum & 8 & 18 \\
\hline FOTL & Vacuum & 8 & 19 \\
\hline FOTL & Vacuum & 8 & 20 \\
\hline FOTL & Vacuum & 8 & 21 \\
\hline FOTL & Vacuum & 8 & 22 \\
\hline FOTL & Vacuum & 8 & 23 \\
\hline FOTL & Vacuum & 8 & 24 \\
\hline FOTL & Vacuum & 8 & 25 \\
\hline FOTL & Vacuum & 9 & 1 \\
\hline FOTL & Vacuum & 9 & 2 \\
\hline
\end{tabular}




\begin{tabular}{|c|c|c|c|c|}
\hline FOTL & Vacuum & 9 & 3 & 17 \\
\hline FOTL & Vacuum & 9 & 4 & 12 \\
\hline FOTL & Vacuum & 9 & 5 & 2 \\
\hline FOTL & Vacuum & 9 & 6 & 5 \\
\hline FOTL & Vacuum & 9 & 7 & 12 \\
\hline FOTL & Vacuum & 9 & 8 & 7 \\
\hline FOTL & Vacuum & 9 & 9 & 38 \\
\hline FOTL & Vacuum & 9 & 10 & 4 \\
\hline FOTL & Vacuum & 9 & 11 & 6 \\
\hline FOTL & Vacuum & 9 & 12 & 26 \\
\hline FOTL & Vacuum & 9 & 13 & 3 \\
\hline FOTL & Vacuum & 9 & 14 & 129 \\
\hline FOTL & Vacuum & 10 & 1 & 9 \\
\hline FOTL & Vacuum & 10 & 2 & 8 \\
\hline FOTL & Vacuum & 10 & 3 & 19 \\
\hline FOTL & Vacuum & 10 & 4 & 7 \\
\hline FOTL & Vacuum & 10 & 5 & 17 \\
\hline FOTL & Vacuum & 10 & 6 & 15 \\
\hline FOTL & Vacuum & 10 & 7 & 7 \\
\hline FOTL & Vacuum & 10 & 8 & 5 \\
\hline FOTL & Vacuum & 10 & 9 & 8 \\
\hline FOTL & Vacuum & 11 & 1 & 6 \\
\hline FOTL & Vacuum & 11 & 2 & 3 \\
\hline FOTL & Vacuum & 11 & 3 & 7 \\
\hline FOTL & Vacuum & 11 & 4 & 4 \\
\hline FOTL & Vacuum & 11 & 5 & 6 \\
\hline FOTL & Vacuum & 11 & 6 & 1 \\
\hline FOTL & Vacuum & 11 & 7 & 2 \\
\hline FOTL & Vacuum & 11 & 8 & 1 \\
\hline FOTL & Vacuum & 12 & 1 & 13 \\
\hline FOTL & Vacuum & 12 & 2 & 3 \\
\hline FOTL & Vacuum & 12 & 3 & 1 \\
\hline FOTL & Vacuum & 12 & 4 & 4 \\
\hline FOTL & Vacuum & 12 & 5 & 5 \\
\hline FOTL & Vacuum & 12 & 6 & 7 \\
\hline FOTL & Vacuum & 12 & 7 & 5 \\
\hline FOTL & Vacuum & 12 & 8 & 10 \\
\hline FOTL & Vacuum & 12 & 9 & 7 \\
\hline FOTL & Vacuum & 12 & 10 & 5 \\
\hline FOTL & Vacuum & 12 & 11 & 2 \\
\hline FOTL & Vacuum & 12 & 12 & 2 \\
\hline FOTL & Vacuum & 13 & 1 & 3 \\
\hline FOTL & Vacuum & 13 & 2 & 4 \\
\hline FOTL & Vacuum & 13 & 3 & 6 \\
\hline FOTL & Vacuum & 13 & 4 & 81 \\
\hline
\end{tabular}




\begin{tabular}{|c|c|c|c|c|}
\hline FOTL & Vacuum & 13 & 5 & 7 \\
\hline FOTL & Vacuum & 13 & 6 & 7 \\
\hline FOTL & Vacuum & 13 & 7 & 7 \\
\hline FOTL & Vacuum & 13 & 8 & 11 \\
\hline FOTL & Vacuum & 13 & 9 & 3 \\
\hline FOTL & Vacuum & 14 & 1 & 2 \\
\hline FOTL & Vacuum & 14 & 2 & 6 \\
\hline FOTL & Vacuum & 14 & 3 & 10 \\
\hline FOTL & Vacuum & 15 & 1 & 5 \\
\hline FOTL & Vacuum & 15 & 2 & 28 \\
\hline FOTL & Vacuum & 15 & 3 & 1 \\
\hline FOTL & Vacuum & 15 & 4 & 2 \\
\hline FOTL & Vacuum & 15 & 5 & 23 \\
\hline FOTL & Vacuum & 15 & 6 & 19 \\
\hline FOTL & Vacuum & 15 & 7 & 5 \\
\hline FOTL & Vacuum & 15 & 8 & 4 \\
\hline FOTL & Vacuum & 15 & 9 & 6 \\
\hline FOTL & Vacuum & 15 & 10 & 5 \\
\hline FOTL & Vacuum & 15 & 11 & 6 \\
\hline FOTL & Vacuum & 15 & 12 & 21 \\
\hline FOTL & Vacuum & 16 & 1 & 21 \\
\hline FOTL & Vacuum & 16 & 2 & 1 \\
\hline FOTL & Vacuum & 16 & 3 & 8 \\
\hline FOTL & Vacuum & 16 & 4 & 4 \\
\hline FOTL & Vacuum & 16 & 5 & 4 \\
\hline FOTL & Vacuum & 16 & 6 & 2 \\
\hline FOTL & Vacuum & 16 & 7 & 20 \\
\hline FOTL & Vacuum & 16 & 8 & 3 \\
\hline FOTL & Vacuum & 16 & 9 & 4 \\
\hline FOTL & Vacuum & 16 & 10 & 63 \\
\hline FOTL & Vacuum & 16 & 11 & 96 \\
\hline FOTL & Vacuum & 16 & 12 & 69 \\
\hline FOTL & Vacuum & 17 & 1 & 44 \\
\hline FOTL & Vacuum & 17 & 2 & 91 \\
\hline FOTL & Vacuum & 17 & 3 & 67 \\
\hline FOTL & Vacuum & 17 & 4 & 139 \\
\hline FOTL & Vacuum & 17 & 5 & 57 \\
\hline FOTL & Vacuum & 18 & 1 & 49 \\
\hline FOTL & Vacuum & 18 & 2 & 48 \\
\hline FOTL & Vacuum & 18 & 3 & 79 \\
\hline FOTL & Vacuum & 18 & 4 & 7 \\
\hline FOTL & Vacuum & 18 & 5 & 46 \\
\hline FOTL & Vacuum & 18 & 6 & 20 \\
\hline FOTL & Vacuum & 18 & 7 & 10 \\
\hline FOTL & Vacuum & 18 & 8 & 14 \\
\hline
\end{tabular}




\begin{tabular}{|c|c|}
\hline FOTL & Vacuum \\
\hline FOTL & Vacuum \\
\hline FOTL & Vacuu \\
\hline FOTL & Vacuu \\
\hline FOTL & Vacuu \\
\hline FOTL & Vacuu \\
\hline FOTL & Vacuu \\
\hline FOTL & Vacuu \\
\hline FOTL & Vacuu \\
\hline FOTL & Vacuu \\
\hline FOTL & Vacuu \\
\hline FOTL & Vacuu \\
\hline FOTL & Vacuu \\
\hline FOTL & Vacuu \\
\hline FOTL & Vacuu \\
\hline FOTL & Vacuu \\
\hline FOTL & Vacuu \\
\hline FOTL & Vacuu \\
\hline FOTL & Vacuur \\
\hline FOTL & Vacuur \\
\hline FOTL & Vacuu \\
\hline FOTL & Vacuu \\
\hline FOTL & Vacuur \\
\hline FOTL & Vacuun \\
\hline FOTL & Vacuu \\
\hline FOTL & Vacuur \\
\hline FOTL & Vacuum \\
\hline FOTL & Vacuu \\
\hline FOTL & Vacuu \\
\hline FOTL & Vacuu \\
\hline FOTL & Vacuur \\
\hline FOTL & Vacuu \\
\hline FOTL & Vacuu \\
\hline FOTL & Vacuu \\
\hline FOTL & Vacuu \\
\hline FOTL & Vacuu \\
\hline FOTL & Vacuur \\
\hline FOTL & Vacuu \\
\hline FOTL & Vacul \\
\hline FOTL & Vacuu \\
\hline FOTL & Vacuu \\
\hline FOTL & Vacuur \\
\hline FOTL & Vacuu \\
\hline FOTL & Vacul \\
\hline FOTL & Vacuur \\
\hline
\end{tabular}

18

18

18

18

18

18

19

19

19

19

20

20

20

20

20

21

21

21

21

23

23

23

23

23

23

23

23

23

23

23

23

24

24

24

24

24

24

24

24

24

24

24

25

25

25
$9 \quad 7$

$10 \quad 9$

114

$12 \quad 8$

$13 \quad 32$

$14 \quad 14$

18

$2 \quad 22$

313

$4 \quad 17$

132

219

320

$4 \quad 11$

$5 \quad 21$

178

$2 \quad 14$

39

43

19

269

39

$4 \quad 16$

510

$6 \quad 12$

73

$8 \quad 59$

$9 \quad 19$

$10 \quad 17$

$11 \quad 19$

126

15

26

311

$4 \quad 1$

52

$6 \quad 1$

$7 \quad 11$

$8 \quad 7$

94

$10 \quad 87$

$11 \quad 12$

162

26

36 


\begin{tabular}{|c|c|c|c|c|}
\hline FOTL & Vacuum & 25 & 4 & 2 \\
\hline FOTL & Vacuum & 25 & 5 & 10 \\
\hline FOTL & Vacuum & 25 & 6 & 8 \\
\hline FOTL & Vacuum & 25 & 7 & 82 \\
\hline FOTL & Vacuum & 26 & 1 & 7 \\
\hline FOTL & Vacuum & 26 & 2 & 25 \\
\hline FOTL & Vacuum & 26 & 3 & 12 \\
\hline FOTL & Vacuum & 26 & 4 & 34 \\
\hline FOTL & Vacuum & 26 & 5 & 16 \\
\hline FOTL & Vacuum & 26 & 6 & 15 \\
\hline FOTL & Vacuum & 26 & 7 & 24 \\
\hline FOTL & Vacuum & 26 & 8 & 13 \\
\hline FOTL & Vacuum & 26 & 9 & 64 \\
\hline FOTL & Vacuum & 26 & 10 & 16 \\
\hline FOTL & Vacuum & 26 & 11 & 17 \\
\hline FOTL & Vacuum & 26 & 12 & 3 \\
\hline FOTL & Vacuum & 26 & 13 & 9 \\
\hline FOTL & Vacuum & 26 & 14 & 16 \\
\hline FOTL & Vacuum & 26 & 15 & 9 \\
\hline FOTL & Vacuum & 27 & 1 & 16 \\
\hline FOTL & Vacuum & 27 & 2 & 26 \\
\hline FOTL & Vacuum & 27 & 3 & 16 \\
\hline FOTL & Vacuum & 27 & 4 & 61 \\
\hline FOTL & Vacuum & 27 & 5 & 52 \\
\hline FOTL & Vacuum & 28 & 1 & 60 \\
\hline FOTL & Vacuum & 28 & 2 & 70 \\
\hline FOTL & Vacuum & 28 & 3 & 18 \\
\hline FOTL & Vacuum & 28 & 4 & 86 \\
\hline FOTL & Vacuum & 28 & 5 & 71 \\
\hline FOTL & Vacuum & 29 & 1 & 58 \\
\hline FOTL & Vacuum & 29 & 2 & 74 \\
\hline FOTL & Vacuum & 29 & 3 & 80 \\
\hline FOTL & Vacuum & 29 & 4 & 73 \\
\hline FOTL & Vacuum & 29 & 5 & 38 \\
\hline FOTL & Vacuum & 29 & 6 & 74 \\
\hline FOTL & Vacuum & 29 & 7 & 77 \\
\hline FOTL & Vacuum & 29 & 8 & 52 \\
\hline FOTL & Vacuum & 29 & 9 & 80 \\
\hline FOTL & Vacuum & 29 & 10 & 62 \\
\hline FOTL & Vacuum & 30 & 1 & 82 \\
\hline FOTL & Vacuum & 30 & 2 & 32 \\
\hline FOTL & Vacuum & 30 & 3 & 93 \\
\hline FOTL & Vacuum & 30 & 4 & 7 \\
\hline FOTL & Vacuum & 30 & 5 & 58 \\
\hline FOTL & Vacuum & 30 & 6 & 57 \\
\hline
\end{tabular}




\begin{tabular}{|c|c|c|c|c|}
\hline FOTL & Vacuum & 30 & 7 & 104 \\
\hline FOTL & Vacuum & 30 & 8 & 46 \\
\hline FOTL & Vacuum & 30 & 9 & 65 \\
\hline FOTL & Vacuum & 30 & 10 & 75 \\
\hline FOTL & Vacuum & 30 & 11 & 99 \\
\hline FOTL & Vacuum & 30 & 12 & 100 \\
\hline FOTL & Vacuum & 30 & 13 & 52 \\
\hline FOTL & Vacuum & 30 & 14 & 71 \\
\hline FOTL & Vacuum & 30 & 15 & 44 \\
\hline FOTL & Vacuum & 30 & 16 & 98 \\
\hline FOTL & Vacuum & 31 & 1 & 46 \\
\hline FOTL & Vacuum & 31 & 2 & 92 \\
\hline FOTL & Vacuum & 31 & 3 & 64 \\
\hline FOTL & Vacuum & 31 & 4 & 34 \\
\hline FOTL & Vacuum & 31 & 5 & 51 \\
\hline FOTL & Vacuum & 32 & 1 & 49 \\
\hline FOTL & Vacuum & 32 & 2 & 15 \\
\hline FOTL & Vacuum & 32 & 3 & 99 \\
\hline FOTL & Vacuum & 32 & 4 & 6 \\
\hline FOTL & Vacuum & 32 & 5 & 162 \\
\hline FOTL & Vacuum & 32 & 6 & 23 \\
\hline FOTL & Vacuum & 32 & 7 & 72 \\
\hline FOTL & Vacuum & 32 & 8 & 64 \\
\hline FOTL & Vacuum & 32 & 9 & 55 \\
\hline FOTL & Vacuum & 32 & 10 & 44 \\
\hline FOTL & Vacuum & 33 & 1 & 83 \\
\hline FOTL & Vacuum & 33 & 2 & 33 \\
\hline FOTL & Vacuum & 33 & 3 & 15 \\
\hline FOTL & Vacuum & 33 & 4 & 88 \\
\hline FOTL & Vacuum & 33 & 5 & 8 \\
\hline FOTL & Vacuum & 33 & 6 & 34 \\
\hline FOTL & Vacuum & 33 & 7 & 33 \\
\hline FOTL & Vacuum & 33 & 8 & 21 \\
\hline FOTL & Vacuum & 33 & 9 & 53 \\
\hline FOTL & Vacuum & 33 & 10 & 86 \\
\hline FOTL & Vacuum & 33 & 11 & 56 \\
\hline FOTL & Vacuum & 33 & 12 & 24 \\
\hline FOTL & Vacuum & 34 & 1 & 53 \\
\hline FOTL & Vacuum & 34 & 2 & 30 \\
\hline FOTL & Vacuum & 34 & 3 & 51 \\
\hline FOTL & Vacuum & 34 & 4 & 28 \\
\hline FOTL & Vacuum & 34 & 5 & 41 \\
\hline FOTL & Vacuum & 34 & 6 & 46 \\
\hline FOTL & Vacuum & 34 & 7 & 52 \\
\hline FOTL & Vacuum & 34 & 8 & 58 \\
\hline
\end{tabular}




\begin{tabular}{|c|c|c|c|c|}
\hline FOTL & Vacuum & 34 & 9 & 45 \\
\hline FOTL & Vacuum & 34 & 10 & 87 \\
\hline FOTL & Vacuum & 34 & 11 & 14 \\
\hline FOTL & Vacuum & 34 & 12 & 18 \\
\hline FOTL & Vacuum & 35 & 1 & 19 \\
\hline FOTL & Vacuum & 35 & 2 & 17 \\
\hline FOTL & Vacuum & 35 & 3 & 37 \\
\hline FOTL & Vacuum & 35 & 4 & 7 \\
\hline FOTL & Vacuum & 35 & 5 & 65 \\
\hline FOTL & Vacuum & 35 & 6 & 82 \\
\hline FOTL & Vacuum & 35 & 7 & 22 \\
\hline FOTL & Vacuum & 35 & 8 & 63 \\
\hline FOTL & Vacuum & 35 & 9 & 55 \\
\hline FOTL & Vacuum & 35 & 10 & 39 \\
\hline FOTL & Vacuum & 35 & 11 & 106 \\
\hline FOTL & Vacuum & 35 & 12 & 58 \\
\hline FOTL & Vacuum & 35 & 13 & 62 \\
\hline FOTL & Vacuum & 35 & 14 & 83 \\
\hline FOTL & Vacuum & 35 & 15 & 58 \\
\hline FOTL & Vacuum & 35 & 16 & 59 \\
\hline Hanes & TapeLift & 1 & 1 & 36 \\
\hline Hanes & TapeLift & 1 & 2 & 19 \\
\hline Hanes & TapeLift & 1 & 3 & 48 \\
\hline Hanes & TapeLift & 1 & 4 & 37 \\
\hline Hanes & TapeLift & 1 & 5 & 39 \\
\hline Hanes & TapeLift & 1 & 6 & 67 \\
\hline Hanes & TapeLift & 1 & 7 & 24 \\
\hline Hanes & TapeLift & 1 & 8 & 30 \\
\hline Hanes & TapeLift & 1 & 9 & 24 \\
\hline Hanes & TapeLift & 1 & 10 & 28 \\
\hline Hanes & TapeLift & 1 & 11 & 33 \\
\hline Hanes & TapeLift & 1 & 12 & 40 \\
\hline Hanes & TapeLift & 1 & 13 & 20 \\
\hline Hanes & TapeLift & 1 & 14 & 16 \\
\hline Hanes & TapeLift & 1 & 15 & 13 \\
\hline Hanes & TapeLift & 1 & 16 & 59 \\
\hline Hanes & TapeLift & 1 & 17 & 17 \\
\hline Hanes & TapeLift & 1 & 18 & 19 \\
\hline Hanes & TapeLift & 1 & 19 & 62 \\
\hline Hanes & TapeLift & 1 & 20 & 22 \\
\hline Hanes & TapeLift & 1 & 21 & 59 \\
\hline Hanes & TapeLift & 1 & 22 & 32 \\
\hline Hanes & TapeLift & 1 & 23 & 38 \\
\hline Hanes & TapeLift & 1 & 24 & 67 \\
\hline Hanes & TapeLift & 1 & 25 & 52 \\
\hline
\end{tabular}




\begin{tabular}{|c|c|c|c|c|}
\hline Hanes & TapeLift & 1 & 26 & 34 \\
\hline Hanes & TapeLift & 1 & 27 & 55 \\
\hline Hanes & TapeLift & 1 & 28 & 65 \\
\hline Hanes & TapeLift & 1 & 29 & 43 \\
\hline Hanes & TapeLift & 1 & 30 & 50 \\
\hline Hanes & TapeLift & 1 & 31 & 35 \\
\hline Hanes & TapeLift & 2 & 1 & 63 \\
\hline Hanes & TapeLift & 2 & 2 & 45 \\
\hline Hanes & TapeLift & 2 & 3 & 73 \\
\hline Hanes & TapeLift & 2 & 4 & 58 \\
\hline Hanes & TapeLift & 2 & 5 & 61 \\
\hline Hanes & TapeLift & 2 & 6 & 73 \\
\hline Hanes & TapeLift & 2 & 7 & 70 \\
\hline Hanes & TapeLift & 2 & 8 & 86 \\
\hline Hanes & TapeLift & 2 & 9 & 107 \\
\hline Hanes & TapeLift & 2 & 10 & 86 \\
\hline Hanes & TapeLift & 2 & 11 & 48 \\
\hline Hanes & TapeLift & 2 & 12 & 45 \\
\hline Hanes & TapeLift & 2 & 13 & 70 \\
\hline Hanes & TapeLift & 2 & 14 & 114 \\
\hline Hanes & TapeLift & 2 & 15 & 67 \\
\hline Hanes & TapeLift & 2 & 16 & 93 \\
\hline Hanes & TapeLift & 2 & 17 & 72 \\
\hline Hanes & TapeLift & 2 & 18 & 81 \\
\hline Hanes & TapeLift & 2 & 19 & 78 \\
\hline Hanes & TapeLift & 2 & 20 & 74 \\
\hline Hanes & TapeLift & 2 & 21 & 85 \\
\hline Hanes & TapeLift & 2 & 22 & 58 \\
\hline Hanes & TapeLift & 2 & 23 & 86 \\
\hline Hanes & TapeLift & 2 & 24 & 47 \\
\hline Hanes & TapeLift & 2 & 25 & 57 \\
\hline Hanes & TapeLift & 2 & 26 & 75 \\
\hline Hanes & TapeLift & 2 & 27 & 95 \\
\hline Hanes & TapeLift & 2 & 28 & 68 \\
\hline Hanes & TapeLift & 2 & 29 & 70 \\
\hline Hanes & TapeLift & 2 & 30 & 77 \\
\hline Hanes & TapeLift & 2 & 31 & 67 \\
\hline Hanes & TapeLift & 2 & 32 & 66 \\
\hline Hanes & TapeLift & 2 & 33 & 65 \\
\hline Hanes & TapeLift & 2 & 34 & 71 \\
\hline Hanes & TapeLift & 2 & 35 & 38 \\
\hline Hanes & TapeLift & 2 & 36 & 68 \\
\hline Hanes & TapeLift & 2 & 37 & 75 \\
\hline Hanes & TapeLift & 2 & 38 & 71 \\
\hline Hanes & TapeLift & 2 & 39 & 79 \\
\hline
\end{tabular}




\begin{tabular}{|c|c|c|c|c|}
\hline Hanes & TapeLift & 2 & 40 & 48 \\
\hline Hanes & TapeLift & 2 & 41 & 38 \\
\hline Hanes & TapeLift & 2 & 42 & 22 \\
\hline Hanes & TapeLift & 2 & 43 & 53 \\
\hline Hanes & TapeLift & 2 & 44 & 24 \\
\hline Hanes & TapeLift & 2 & 45 & 146 \\
\hline Hanes & TapeLift & 2 & 46 & 92 \\
\hline Hanes & TapeLift & 2 & 47 & 55 \\
\hline Hanes & TapeLift & 2 & 48 & 46 \\
\hline Hanes & TapeLift & 2 & 49 & 70 \\
\hline Hanes & TapeLift & 2 & 50 & 51 \\
\hline Hanes & TapeLift & 2 & 51 & 87 \\
\hline Hanes & TapeLift & 3 & 1 & 73 \\
\hline Hanes & TapeLift & 3 & 2 & 98 \\
\hline Hanes & TapeLift & 3 & 3 & 90 \\
\hline Hanes & TapeLift & 3 & 4 & 84 \\
\hline Hanes & TapeLift & 3 & 5 & 111 \\
\hline Hanes & TapeLift & 3 & 6 & 109 \\
\hline Hanes & TapeLift & 3 & 7 & 92 \\
\hline Hanes & TapeLift & 3 & 8 & 84 \\
\hline Hanes & TapeLift & 3 & 9 & 81 \\
\hline Hanes & TapeLift & 3 & 10 & 90 \\
\hline Hanes & TapeLift & 3 & 11 & 66 \\
\hline Hanes & TapeLift & 3 & 12 & 38 \\
\hline Hanes & TapeLift & 3 & 13 & 82 \\
\hline Hanes & TapeLift & 3 & 14 & 89 \\
\hline Hanes & TapeLift & 3 & 15 & 40 \\
\hline Hanes & TapeLift & 3 & 16 & 110 \\
\hline Hanes & TapeLift & 3 & 17 & 130 \\
\hline Hanes & TapeLift & 3 & 18 & 77 \\
\hline Hanes & TapeLift & 3 & 19 & 106 \\
\hline Hanes & TapeLift & 3 & 20 & 87 \\
\hline Hanes & TapeLift & 3 & 21 & 82 \\
\hline Hanes & TapeLift & 3 & 22 & 74 \\
\hline Hanes & TapeLift & 3 & 23 & 108 \\
\hline Hanes & TapeLift & 3 & 24 & 89 \\
\hline Hanes & TapeLift & 3 & 25 & 83 \\
\hline Hanes & TapeLift & 3 & 26 & 109 \\
\hline Hanes & TapeLift & 3 & 27 & 100 \\
\hline Hanes & TapeLift & 3 & 28 & 62 \\
\hline Hanes & TapeLift & 3 & 29 & 61 \\
\hline Hanes & TapeLift & 3 & 30 & 104 \\
\hline Hanes & TapeLift & 3 & 31 & 92 \\
\hline Hanes & TapeLift & 3 & 32 & 89 \\
\hline Hanes & TapeLift & 3 & 33 & 70 \\
\hline
\end{tabular}




\begin{tabular}{|c|c|c|c|}
\hline Hanes & TapeLift & 3 & 34 \\
\hline Hanes & TapeLift & 3 & 35 \\
\hline Hanes & TapeLift & 4 & 1 \\
\hline Hanes & TapeLift & 4 & 2 \\
\hline Hanes & TapeLift & 4 & 3 \\
\hline Hanes & TapeLift & 4 & 4 \\
\hline Hanes & TapeLift & 4 & 5 \\
\hline Hanes & TapeLift & 4 & 6 \\
\hline Hanes & TapeLift & 4 & 7 \\
\hline Hanes & TapeLift & 4 & 8 \\
\hline Hanes & TapeLift & 4 & 9 \\
\hline Hanes & TapeLift & 4 & 10 \\
\hline Hanes & TapeLift & 4 & 11 \\
\hline Hanes & TapeLift & 4 & 12 \\
\hline Hanes & TapeLift & 4 & 13 \\
\hline Hanes & TapeLift & 4 & 14 \\
\hline Hanes & TapeLift & 4 & 15 \\
\hline Hanes & TapeLift & 4 & 16 \\
\hline Hanes & TapeLift & 4 & 17 \\
\hline Hanes & TapeLift & 4 & 18 \\
\hline Hanes & TapeLift & 4 & 19 \\
\hline Hanes & TapeLift & 4 & 20 \\
\hline Hanes & TapeLift & 4 & 21 \\
\hline Hanes & TapeLift & 4 & 22 \\
\hline Hanes & TapeLift & 4 & 23 \\
\hline Hanes & TapeLift & 4 & 24 \\
\hline Hanes & TapeLift & 4 & 25 \\
\hline Hanes & TapeLift & 4 & 26 \\
\hline Hanes & TapeLift & 5 & 1 \\
\hline Hanes & TapeLift & 5 & 2 \\
\hline Hanes & TapeLift & 5 & 3 \\
\hline Hanes & TapeLift & 5 & 4 \\
\hline Hanes & TapeLift & 5 & 5 \\
\hline Hanes & TapeLift & 5 & 6 \\
\hline Hanes & TapeLift & 5 & 7 \\
\hline Hanes & TapeLift & 5 & 8 \\
\hline Hanes & TapeLift & 5 & 9 \\
\hline Hanes & TapeLift & 5 & 10 \\
\hline Hanes & TapeLift & 5 & 11 \\
\hline Hanes & TapeLift & 5 & 12 \\
\hline Hanes & TapeLift & 5 & 13 \\
\hline Hanes & TapeLift & 5 & 14 \\
\hline Hanes & TapeLift & 5 & 15 \\
\hline Hanes & TapeLift & 5 & 16 \\
\hline Hanes & TapeLift & 5 & 17 \\
\hline
\end{tabular}




\begin{tabular}{|c|c|c|c|c|}
\hline Hanes & TapeLift & 5 & 18 & 56 \\
\hline Hanes & TapeLift & 5 & 19 & 59 \\
\hline Hanes & TapeLift & 5 & 20 & 80 \\
\hline Hanes & TapeLift & 5 & 21 & 55 \\
\hline Hanes & TapeLift & 5 & 22 & 59 \\
\hline Hanes & TapeLift & 5 & 23 & 56 \\
\hline Hanes & TapeLift & 5 & 24 & 59 \\
\hline Hanes & TapeLift & 5 & 25 & 88 \\
\hline Hanes & TapeLift & 5 & 26 & 86 \\
\hline Hanes & TapeLift & 5 & 27 & 52 \\
\hline Hanes & TapeLift & 5 & 28 & 50 \\
\hline Hanes & TapeLift & 5 & 29 & 50 \\
\hline Hanes & TapeLift & 5 & 30 & 46 \\
\hline Hanes & TapeLift & 5 & 31 & 55 \\
\hline Hanes & TapeLift & 5 & 32 & 80 \\
\hline Hanes & TapeLift & 5 & 33 & 72 \\
\hline Hanes & TapeLift & 5 & 34 & 59 \\
\hline Hanes & TapeLift & 5 & 35 & 93 \\
\hline Hanes & TapeLift & 5 & 36 & 56 \\
\hline Hanes & TapeLift & 5 & 37 & 64 \\
\hline Hanes & TapeLift & 5 & 38 & 88 \\
\hline Hanes & TapeLift & 5 & 39 & 53 \\
\hline Hanes & TapeLift & 5 & 40 & 96 \\
\hline Hanes & TapeLift & 6 & 1 & 84 \\
\hline Hanes & TapeLift & 6 & 2 & 56 \\
\hline Hanes & TapeLift & 6 & 3 & 65 \\
\hline Hanes & TapeLift & 6 & 4 & 89 \\
\hline Hanes & TapeLift & 6 & 5 & 154 \\
\hline Hanes & TapeLift & 6 & 6 & 99 \\
\hline Hanes & TapeLift & 6 & 7 & 80 \\
\hline Hanes & TapeLift & 6 & 8 & 68 \\
\hline Hanes & TapeLift & 6 & 9 & 81 \\
\hline Hanes & TapeLift & 6 & 10 & 56 \\
\hline Hanes & TapeLift & 6 & 11 & 50 \\
\hline Hanes & TapeLift & 6 & 12 & 47 \\
\hline Hanes & TapeLift & 6 & 13 & 52 \\
\hline Hanes & TapeLift & 6 & 14 & 61 \\
\hline Hanes & TapeLift & 6 & 15 & 94 \\
\hline Hanes & TapeLift & 6 & 16 & 102 \\
\hline Hanes & TapeLift & 6 & 17 & 53 \\
\hline Hanes & TapeLift & 6 & 18 & 55 \\
\hline Hanes & TapeLift & 6 & 19 & 79 \\
\hline Hanes & TapeLift & 6 & 20 & 81 \\
\hline Hanes & TapeLift & 6 & 21 & 79 \\
\hline Hanes & TapeLift & 6 & 22 & 81 \\
\hline
\end{tabular}




\begin{tabular}{|c|c|c|c|c|}
\hline Hanes & TapeLift & 6 & 23 & 85 \\
\hline Hanes & TapeLift & 6 & 24 & 74 \\
\hline Hanes & TapeLift & 6 & 25 & 76 \\
\hline Hanes & TapeLift & 6 & 26 & 79 \\
\hline Hanes & TapeLift & 6 & 27 & 58 \\
\hline Hanes & TapeLift & 6 & 28 & 29 \\
\hline Hanes & TapeLift & 6 & 29 & 31 \\
\hline Hanes & TapeLift & 6 & 30 & 61 \\
\hline Hanes & TapeLift & 7 & 1 & 77 \\
\hline Hanes & TapeLift & 7 & 2 & 73 \\
\hline Hanes & TapeLift & 7 & 3 & 48 \\
\hline Hanes & TapeLift & 7 & 4 & 58 \\
\hline Hanes & TapeLift & 7 & 5 & 36 \\
\hline Hanes & TapeLift & 7 & 6 & 42 \\
\hline Hanes & TapeLift & 7 & 7 & 46 \\
\hline Hanes & TapeLift & 7 & 8 & 44 \\
\hline Hanes & TapeLift & 7 & 9 & 50 \\
\hline Hanes & TapeLift & 7 & 10 & 42 \\
\hline Hanes & TapeLift & 7 & 11 & 36 \\
\hline Hanes & TapeLift & 7 & 12 & 102 \\
\hline Hanes & TapeLift & 7 & 13 & 32 \\
\hline Hanes & TapeLift & 7 & 14 & 52 \\
\hline Hanes & TapeLift & 7 & 15 & 55 \\
\hline Hanes & TapeLift & 7 & 16 & 71 \\
\hline Hanes & TapeLift & 7 & 17 & 105 \\
\hline Hanes & TapeLift & 7 & 18 & 78 \\
\hline Hanes & TapeLift & 7 & 19 & 64 \\
\hline Hanes & TapeLift & 8 & 1 & 82 \\
\hline Hanes & TapeLift & 8 & 2 & 98 \\
\hline Hanes & TapeLift & 8 & 3 & 87 \\
\hline Hanes & TapeLift & 8 & 4 & 80 \\
\hline Hanes & TapeLift & 8 & 5 & 71 \\
\hline Hanes & TapeLift & 8 & 6 & 75 \\
\hline Hanes & TapeLift & 8 & 7 & 42 \\
\hline Hanes & TapeLift & 8 & 8 & 86 \\
\hline Hanes & TapeLift & 8 & 9 & 46 \\
\hline Hanes & TapeLift & 8 & 10 & 50 \\
\hline Hanes & TapeLift & 8 & 11 & 115 \\
\hline Hanes & TapeLift & 8 & 12 & 74 \\
\hline Hanes & TapeLift & 8 & 13 & 58 \\
\hline Hanes & TapeLift & 8 & 14 & 60 \\
\hline Hanes & TapeLift & 8 & 15 & 53 \\
\hline Hanes & TapeLift & 8 & 16 & 52 \\
\hline Hanes & TapeLift & 8 & 17 & 92 \\
\hline Hanes & TapeLift & 8 & 18 & 47 \\
\hline
\end{tabular}




\begin{tabular}{|c|c|c|c|c|}
\hline Hanes & TapeLift & 8 & 19 & 74 \\
\hline Hanes & TapeLift & 8 & 20 & 92 \\
\hline Hanes & TapeLift & 8 & 21 & 87 \\
\hline Hanes & TapeLift & 8 & 22 & 66 \\
\hline Hanes & TapeLift & 8 & 23 & 99 \\
\hline Hanes & TapeLift & 8 & 24 & 90 \\
\hline Hanes & TapeLift & 8 & 25 & 102 \\
\hline Hanes & TapeLift & 8 & 26 & 94 \\
\hline Hanes & TapeLift & 8 & 27 & 42 \\
\hline Hanes & TapeLift & 8 & 28 & 58 \\
\hline Hanes & TapeLift & 8 & 29 & 49 \\
\hline Hanes & TapeLift & 8 & 30 & 57 \\
\hline Hanes & TapeLift & 9 & 1 & 84 \\
\hline Hanes & TapeLift & 9 & 2 & 95 \\
\hline Hanes & TapeLift & 9 & 3 & 110 \\
\hline Hanes & TapeLift & 9 & 4 & 62 \\
\hline Hanes & TapeLift & 9 & 5 & 82 \\
\hline Hanes & TapeLift & 9 & 6 & 51 \\
\hline Hanes & TapeLift & 9 & 7 & 82 \\
\hline Hanes & TapeLift & 9 & 8 & 53 \\
\hline Hanes & TapeLift & 9 & 9 & 98 \\
\hline Hanes & TapeLift & 9 & 10 & 85 \\
\hline Hanes & TapeLift & 9 & 11 & 51 \\
\hline Hanes & TapeLift & 9 & 12 & 72 \\
\hline Hanes & TapeLift & 9 & 13 & 33 \\
\hline Hanes & TapeLift & 9 & 14 & 47 \\
\hline Hanes & TapeLift & 9 & 15 & 59 \\
\hline Hanes & TapeLift & 9 & 16 & 71 \\
\hline Hanes & TapeLift & 9 & 17 & 77 \\
\hline Hanes & TapeLift & 9 & 18 & 91 \\
\hline Hanes & TapeLift & 9 & 19 & 106 \\
\hline Hanes & TapeLift & 9 & 20 & 80 \\
\hline Hanes & TapeLift & 9 & 21 & 42 \\
\hline Hanes & TapeLift & 10 & 1 & 24 \\
\hline Hanes & TapeLift & 10 & 2 & 93 \\
\hline Hanes & TapeLift & 10 & 3 & 60 \\
\hline Hanes & TapeLift & 10 & 4 & 79 \\
\hline Hanes & TapeLift & 10 & 5 & 81 \\
\hline Hanes & TapeLift & 10 & 6 & 67 \\
\hline Hanes & TapeLift & 10 & 7 & 59 \\
\hline Hanes & TapeLift & 10 & 8 & 89 \\
\hline Hanes & TapeLift & 10 & 9 & 17 \\
\hline Hanes & TapeLift & 10 & 10 & 48 \\
\hline Hanes & TapeLift & 10 & 11 & 43 \\
\hline Hanes & TapeLift & 10 & 12 & 53 \\
\hline
\end{tabular}




\begin{tabular}{|c|c|c|c|c|}
\hline Hanes & TapeLift & 10 & 13 & 41 \\
\hline Hanes & TapeLift & 10 & 14 & 53 \\
\hline Hanes & TapeLift & 10 & 15 & 54 \\
\hline Hanes & TapeLift & 10 & 16 & 48 \\
\hline Hanes & TapeLift & 10 & 17 & 54 \\
\hline Hanes & TapeLift & 10 & 18 & 63 \\
\hline Hanes & TapeLift & 10 & 19 & 49 \\
\hline Hanes & TapeLift & 10 & 20 & 62 \\
\hline Hanes & TapeLift & 10 & 21 & 35 \\
\hline Hanes & TapeLift & 11 & 1 & 50 \\
\hline Hanes & TapeLift & 11 & 2 & 42 \\
\hline Hanes & TapeLift & 11 & 3 & 40 \\
\hline Hanes & TapeLift & 11 & 4 & 35 \\
\hline Hanes & TapeLift & 11 & 5 & 37 \\
\hline Hanes & TapeLift & 11 & 6 & 48 \\
\hline Hanes & TapeLift & 11 & 7 & 55 \\
\hline Hanes & TapeLift & 11 & 8 & 22 \\
\hline Hanes & TapeLift & 11 & 9 & 63 \\
\hline Hanes & TapeLift & 11 & 10 & 83 \\
\hline Hanes & TapeLift & 11 & 11 & 40 \\
\hline Hanes & TapeLift & 11 & 12 & 33 \\
\hline Hanes & TapeLift & 11 & 13 & 78 \\
\hline Hanes & TapeLift & 11 & 14 & 39 \\
\hline Hanes & TapeLift & 12 & 1 & 17 \\
\hline Hanes & TapeLift & 12 & 2 & 40 \\
\hline Hanes & TapeLift & 12 & 3 & 52 \\
\hline Hanes & TapeLift & 12 & 4 & 53 \\
\hline Hanes & TapeLift & 12 & 5 & 42 \\
\hline Hanes & TapeLift & 12 & 6 & 47 \\
\hline Hanes & TapeLift & 12 & 7 & 63 \\
\hline Hanes & TapeLift & 12 & 8 & 72 \\
\hline Hanes & TapeLift & 12 & 9 & 31 \\
\hline Hanes & TapeLift & 12 & 10 & 64 \\
\hline Hanes & TapeLift & 12 & 11 & 49 \\
\hline Hanes & TapeLift & 12 & 12 & 29 \\
\hline Hanes & TapeLift & 12 & 13 & 67 \\
\hline Hanes & TapeLift & 12 & 14 & 53 \\
\hline Hanes & TapeLift & 12 & 15 & 42 \\
\hline Hanes & TapeLift & 12 & 16 & 25 \\
\hline Hanes & TapeLift & 12 & 17 & 52 \\
\hline Hanes & TapeLift & 13 & 1 & 83 \\
\hline Hanes & TapeLift & 13 & 2 & 25 \\
\hline Hanes & TapeLift & 13 & 3 & 30 \\
\hline Hanes & TapeLift & 13 & 4 & 32 \\
\hline Hanes & TapeLift & 13 & 5 & 22 \\
\hline
\end{tabular}




\begin{tabular}{|c|c|c|c|}
\hline Hanes & TapeLift & 13 & 6 \\
\hline Hanes & TapeLift & 13 & 7 \\
\hline Hanes & TapeLift & 13 & 8 \\
\hline Hanes & TapeLift & 13 & 9 \\
\hline Hanes & TapeLift & 13 & 10 \\
\hline Hanes & TapeLift & 13 & 11 \\
\hline Hanes & TapeLift & 13 & 12 \\
\hline Hanes & TapeLift & 13 & 13 \\
\hline Hanes & TapeLift & 13 & 14 \\
\hline Hanes & TapeLift & 14 & 1 \\
\hline Hanes & TapeLift & 14 & 2 \\
\hline Hanes & TapeLift & 14 & 3 \\
\hline Hanes & TapeLift & 14 & 4 \\
\hline Hanes & TapeLift & 14 & 5 \\
\hline Hanes & TapeLift & 14 & 6 \\
\hline Hanes & TapeLift & 14 & 7 \\
\hline Hanes & TapeLift & 14 & 8 \\
\hline Hanes & TapeLift & 14 & 9 \\
\hline Hanes & TapeLift & 14 & 10 \\
\hline Hanes & TapeLift & 14 & 11 \\
\hline Hanes & TapeLift & 14 & 12 \\
\hline Hanes & TapeLift & 14 & 13 \\
\hline Hanes & TapeLift & 14 & 14 \\
\hline Hanes & TapeLift & 14 & 15 \\
\hline Hanes & TapeLift & 14 & 16 \\
\hline Hanes & TapeLift & 14 & 17 \\
\hline Hanes & TapeLift & 14 & 18 \\
\hline Hanes & TapeLift & 14 & 19 \\
\hline Hanes & TapeLift & 14 & 20 \\
\hline Hanes & TapeLift & 15 & 1 \\
\hline Hanes & TapeLift & 15 & 2 \\
\hline Hanes & TapeLift & 15 & 3 \\
\hline Hanes & TapeLift & 15 & 4 \\
\hline Hanes & TapeLift & 15 & 5 \\
\hline Hanes & TapeLift & 15 & 6 \\
\hline Hanes & TapeLift & 15 & 7 \\
\hline Hanes & TapeLift & 15 & 8 \\
\hline Hanes & TapeLift & 15 & 9 \\
\hline Hanes & TapeLift & 15 & 10 \\
\hline Hanes & TapeLift & 15 & 11 \\
\hline Hanes & TapeLift & 15 & 12 \\
\hline Hanes & TapeLift & 15 & 13 \\
\hline Hanes & TapeLift & 15 & 14 \\
\hline Hanes & TapeLift & 15 & 15 \\
\hline Hanes & TapeLift & 15 & 16 \\
\hline
\end{tabular}




\begin{tabular}{|c|c|c|c|c|}
\hline Hanes & TapeLift & 15 & 17 & 111 \\
\hline Hanes & TapeLift & 15 & 18 & 70 \\
\hline Hanes & TapeLift & 15 & 19 & 127 \\
\hline Hanes & TapeLift & 15 & 20 & 159 \\
\hline Hanes & TapeLift & 16 & 1 & 102 \\
\hline Hanes & TapeLift & 16 & 2 & 72 \\
\hline Hanes & TapeLift & 16 & 3 & 103 \\
\hline Hanes & TapeLift & 16 & 4 & 145 \\
\hline Hanes & TapeLift & 16 & 5 & 141 \\
\hline Hanes & TapeLift & 16 & 6 & 133 \\
\hline Hanes & TapeLift & 16 & 7 & 124 \\
\hline Hanes & TapeLift & 16 & 8 & 109 \\
\hline Hanes & TapeLift & 16 & 9 & 103 \\
\hline Hanes & TapeLift & 16 & 10 & 140 \\
\hline Hanes & TapeLift & 16 & 11 & 108 \\
\hline Hanes & TapeLift & 16 & 12 & 131 \\
\hline Hanes & TapeLift & 16 & 13 & 65 \\
\hline Hanes & TapeLift & 16 & 14 & 110 \\
\hline Hanes & TapeLift & 16 & 15 & 131 \\
\hline Hanes & TapeLift & 16 & 16 & 103 \\
\hline Hanes & TapeLift & 17 & 1 & 92 \\
\hline Hanes & TapeLift & 17 & 2 & 140 \\
\hline Hanes & TapeLift & 17 & 3 & 117 \\
\hline Hanes & TapeLift & 17 & 4 & 126 \\
\hline Hanes & TapeLift & 17 & 5 & 55 \\
\hline Hanes & TapeLift & 17 & 6 & 70 \\
\hline Hanes & TapeLift & 17 & 7 & 85 \\
\hline Hanes & TapeLift & 17 & 8 & 81 \\
\hline Hanes & TapeLift & 17 & 9 & 92 \\
\hline Hanes & TapeLift & 17 & 10 & 78 \\
\hline Hanes & TapeLift & 17 & 11 & 77 \\
\hline Hanes & TapeLift & 17 & 12 & 81 \\
\hline Hanes & TapeLift & 17 & 13 & 119 \\
\hline Hanes & TapeLift & 17 & 14 & 29 \\
\hline Hanes & TapeLift & 17 & 15 & 132 \\
\hline Hanes & TapeLift & 17 & 16 & 114 \\
\hline Hanes & TapeLift & 17 & 17 & 97 \\
\hline Hanes & TapeLift & 17 & 18 & 84 \\
\hline Hanes & TapeLift & 17 & 19 & 116 \\
\hline Hanes & TapeLift & 17 & 20 & 95 \\
\hline Hanes & TapeLift & 17 & 21 & 120 \\
\hline Hanes & TapeLift & 17 & 22 & 154 \\
\hline Hanes & TapeLift & 17 & 23 & 126 \\
\hline Hanes & TapeLift & 17 & 24 & 65 \\
\hline Hanes & TapeLift & 18 & 1 & 101 \\
\hline
\end{tabular}




\begin{tabular}{|c|c|c|c|c|}
\hline Hanes & TapeLift & 18 & 2 & 89 \\
\hline Hanes & TapeLift & 18 & 3 & 80 \\
\hline Hanes & TapeLift & 18 & 4 & 145 \\
\hline Hanes & TapeLift & 18 & 5 & 140 \\
\hline Hanes & TapeLift & 18 & 6 & 120 \\
\hline Hanes & TapeLift & 18 & 7 & 123 \\
\hline Hanes & TapeLift & 18 & 8 & 143 \\
\hline Hanes & TapeLift & 18 & 9 & 110 \\
\hline Hanes & TapeLift & 18 & 10 & 121 \\
\hline Hanes & TapeLift & 18 & 11 & 130 \\
\hline Hanes & TapeLift & 18 & 12 & 136 \\
\hline Hanes & TapeLift & 18 & 13 & 119 \\
\hline Hanes & TapeLift & 18 & 14 & 134 \\
\hline Hanes & TapeLift & 18 & 15 & 118 \\
\hline Hanes & TapeLift & 18 & 16 & 77 \\
\hline Hanes & TapeLift & 18 & 17 & 80 \\
\hline Hanes & TapeLift & 18 & 18 & 85 \\
\hline Hanes & TapeLift & 19 & 1 & 98 \\
\hline Hanes & TapeLift & 19 & 2 & 123 \\
\hline Hanes & TapeLift & 19 & 3 & 130 \\
\hline Hanes & TapeLift & 19 & 4 & 115 \\
\hline Hanes & TapeLift & 19 & 5 & 100 \\
\hline Hanes & TapeLift & 19 & 6 & 127 \\
\hline Hanes & TapeLift & 19 & 7 & 96 \\
\hline Hanes & TapeLift & 19 & 8 & 66 \\
\hline Hanes & TapeLift & 19 & 9 & 62 \\
\hline Hanes & TapeLift & 19 & 10 & 78 \\
\hline Hanes & TapeLift & 19 & 11 & 61 \\
\hline Hanes & TapeLift & 19 & 12 & 104 \\
\hline Hanes & TapeLift & 19 & 13 & 110 \\
\hline Hanes & TapeLift & 19 & 14 & 102 \\
\hline Hanes & TapeLift & 19 & 15 & 133 \\
\hline Hanes & TapeLift & 19 & 16 & 81 \\
\hline Hanes & TapeLift & 19 & 17 & 132 \\
\hline Hanes & TapeLift & 19 & 18 & 105 \\
\hline Hanes & TapeLift & 19 & 19 & 119 \\
\hline Hanes & TapeLift & 19 & 20 & 107 \\
\hline Hanes & TapeLift & 19 & 21 & 123 \\
\hline Hanes & TapeLift & 19 & 22 & 87 \\
\hline Hanes & TapeLift & 19 & 23 & 103 \\
\hline Hanes & TapeLift & 19 & 24 & 62 \\
\hline Hanes & TapeLift & 19 & 25 & 91 \\
\hline Hanes & TapeLift & 19 & 26 & 83 \\
\hline Hanes & TapeLift & 19 & 27 & 59 \\
\hline Hanes & TapeLift & 20 & 1 & 108 \\
\hline
\end{tabular}




\begin{tabular}{|c|c|c|c|c|}
\hline Hanes & TapeLift & 20 & 2 & 90 \\
\hline Hanes & TapeLift & 20 & 3 & 63 \\
\hline Hanes & TapeLift & 20 & 4 & 62 \\
\hline Hanes & TapeLift & 20 & 5 & 66 \\
\hline Hanes & TapeLift & 20 & 6 & 70 \\
\hline Hanes & TapeLift & 20 & 7 & 61 \\
\hline Hanes & TapeLift & 20 & 8 & 127 \\
\hline Hanes & TapeLift & 20 & 9 & 107 \\
\hline Hanes & TapeLift & 20 & 10 & 100 \\
\hline Hanes & TapeLift & 20 & 11 & 83 \\
\hline Hanes & TapeLift & 20 & 12 & 107 \\
\hline Hanes & TapeLift & 20 & 13 & 99 \\
\hline Hanes & TapeLift & 20 & 14 & 147 \\
\hline Hanes & TapeLift & 20 & 15 & 176 \\
\hline Hanes & TapeLift & 20 & 16 & 188 \\
\hline Hanes & TapeLift & 20 & 17 & 104 \\
\hline Hanes & TapeLift & 20 & 18 & 122 \\
\hline Hanes & TapeLift & 20 & 19 & 180 \\
\hline Hanes & TapeLift & 21 & 1 & 158 \\
\hline Hanes & TapeLift & 21 & 2 & 184 \\
\hline Hanes & TapeLift & 21 & 3 & 199 \\
\hline Hanes & TapeLift & 21 & 4 & 114 \\
\hline Hanes & TapeLift & 21 & 5 & 132 \\
\hline Hanes & TapeLift & 21 & 6 & 158 \\
\hline Hanes & TapeLift & 21 & 7 & 185 \\
\hline Hanes & TapeLift & 21 & 8 & 150 \\
\hline Hanes & TapeLift & 21 & 9 & 192 \\
\hline Hanes & TapeLift & 21 & 10 & 233 \\
\hline Hanes & TapeLift & 21 & 11 & 153 \\
\hline Hanes & TapeLift & 21 & 12 & 145 \\
\hline Hanes & TapeLift & 21 & 13 & 165 \\
\hline Hanes & TapeLift & 21 & 14 & 122 \\
\hline Hanes & TapeLift & 21 & 15 & 157 \\
\hline Hanes & TapeLift & 21 & 16 & 185 \\
\hline Hanes & TapeLift & 21 & 17 & 167 \\
\hline Hanes & TapeLift & 21 & 18 & 117 \\
\hline Hanes & TapeLift & 21 & 19 & 165 \\
\hline Hanes & TapeLift & 21 & 20 & 138 \\
\hline Hanes & TapeLift & 21 & 21 & 127 \\
\hline Hanes & TapeLift & 21 & 22 & 158 \\
\hline Hanes & TapeLift & 22 & 1 & 139 \\
\hline Hanes & TapeLift & 22 & 2 & 153 \\
\hline Hanes & TapeLift & 22 & 3 & 159 \\
\hline Hanes & TapeLift & 22 & 4 & 125 \\
\hline Hanes & TapeLift & 22 & 5 & 125 \\
\hline
\end{tabular}




\begin{tabular}{|c|c|c|c|c|}
\hline Hanes & TapeLift & 22 & 6 & 141 \\
\hline Hanes & TapeLift & 22 & 7 & 99 \\
\hline Hanes & TapeLift & 22 & 8 & 113 \\
\hline Hanes & TapeLift & 22 & 9 & 148 \\
\hline Hanes & TapeLift & 22 & 10 & 161 \\
\hline Hanes & TapeLift & 22 & 11 & 94 \\
\hline Hanes & TapeLift & 22 & 12 & 174 \\
\hline Hanes & TapeLift & 22 & 13 & 185 \\
\hline Hanes & TapeLift & 22 & 14 & 137 \\
\hline Hanes & TapeLift & 22 & 15 & 121 \\
\hline Hanes & TapeLift & 22 & 16 & 124 \\
\hline Hanes & TapeLift & 22 & 17 & 134 \\
\hline Hanes & TapeLift & 22 & 18 & 138 \\
\hline Hanes & TapeLift & 22 & 19 & 157 \\
\hline Hanes & TapeLift & 23 & 1 & 120 \\
\hline Hanes & TapeLift & 23 & 2 & 126 \\
\hline Hanes & TapeLift & 23 & 3 & 104 \\
\hline Hanes & TapeLift & 23 & 4 & 107 \\
\hline Hanes & TapeLift & 23 & 5 & 149 \\
\hline Hanes & TapeLift & 23 & 6 & 153 \\
\hline Hanes & TapeLift & 23 & 7 & 140 \\
\hline Hanes & TapeLift & 23 & 8 & 107 \\
\hline Hanes & TapeLift & 23 & 9 & 134 \\
\hline Hanes & TapeLift & 23 & 10 & 187 \\
\hline Hanes & TapeLift & 23 & 11 & 167 \\
\hline Hanes & TapeLift & 23 & 12 & 185 \\
\hline Hanes & TapeLift & 23 & 13 & 207 \\
\hline Hanes & TapeLift & 23 & 14 & 185 \\
\hline Hanes & TapeLift & 23 & 15 & 161 \\
\hline Hanes & TapeLift & 23 & 16 & 121 \\
\hline Hanes & TapeLift & 23 & 17 & 190 \\
\hline Hanes & TapeLift & 23 & 18 & 176 \\
\hline Hanes & TapeLift & 23 & 19 & 196 \\
\hline Hanes & TapeLift & 23 & 20 & 150 \\
\hline Hanes & TapeLift & 23 & 21 & 154 \\
\hline Hanes & TapeLift & 24 & 1 & 123 \\
\hline Hanes & TapeLift & 24 & 2 & 77 \\
\hline Hanes & TapeLift & 24 & 3 & 64 \\
\hline Hanes & TapeLift & 24 & 4 & 178 \\
\hline Hanes & TapeLift & 24 & 5 & 181 \\
\hline Hanes & TapeLift & 24 & 6 & 191 \\
\hline Hanes & TapeLift & 24 & 7 & 167 \\
\hline Hanes & TapeLift & 24 & 8 & 56 \\
\hline Hanes & TapeLift & 24 & 9 & 55 \\
\hline Hanes & TapeLift & 24 & 10 & 80 \\
\hline
\end{tabular}




\begin{tabular}{|c|c|c|c|c|}
\hline Hanes & TapeLift & 24 & 11 & 83 \\
\hline Hanes & TapeLift & 24 & 12 & 26 \\
\hline Hanes & TapeLift & 24 & 13 & 51 \\
\hline Hanes & TapeLift & 24 & 14 & 66 \\
\hline Hanes & TapeLift & 24 & 15 & 53 \\
\hline Hanes & TapeLift & 24 & 16 & 72 \\
\hline Hanes & TapeLift & 24 & 17 & 57 \\
\hline Hanes & TapeLift & 24 & 18 & 96 \\
\hline Hanes & TapeLift & 24 & 19 & 63 \\
\hline Hanes & TapeLift & 24 & 20 & 57 \\
\hline Hanes & TapeLift & 24 & 21 & 49 \\
\hline Hanes & TapeLift & 24 & 22 & 36 \\
\hline Hanes & TapeLift & 24 & 23 & 37 \\
\hline Hanes & TapeLift & 24 & 24 & 11 \\
\hline Hanes & TapeLift & 24 & 25 & 34 \\
\hline Hanes & TapeLift & 24 & 26 & 38 \\
\hline Hanes & TapeLift & 24 & 27 & 70 \\
\hline Hanes & TapeLift & 24 & 28 & 85 \\
\hline Hanes & TapeLift & 24 & 29 & 63 \\
\hline Hanes & TapeLift & 25 & 1 & 98 \\
\hline Hanes & TapeLift & 25 & 2 & 94 \\
\hline Hanes & TapeLift & 25 & 3 & 84 \\
\hline Hanes & TapeLift & 25 & 4 & 59 \\
\hline Hanes & TapeLift & 25 & 5 & 45 \\
\hline Hanes & TapeLift & 25 & 6 & 49 \\
\hline Hanes & TapeLift & 25 & 7 & 114 \\
\hline Hanes & TapeLift & 25 & 8 & 110 \\
\hline Hanes & TapeLift & 25 & 9 & 109 \\
\hline Hanes & TapeLift & 25 & 10 & 122 \\
\hline Hanes & TapeLift & 25 & 11 & 60 \\
\hline Hanes & TapeLift & 25 & 12 & 91 \\
\hline Hanes & TapeLift & 25 & 13 & 110 \\
\hline Hanes & TapeLift & 25 & 14 & 91 \\
\hline Hanes & TapeLift & 25 & 15 & 66 \\
\hline Hanes & TapeLift & 26 & 1 & 64 \\
\hline Hanes & TapeLift & 26 & 2 & 72 \\
\hline Hanes & TapeLift & 26 & 3 & 77 \\
\hline Hanes & TapeLift & 26 & 4 & 65 \\
\hline Hanes & TapeLift & 26 & 5 & 99 \\
\hline Hanes & TapeLift & 26 & 6 & 93 \\
\hline Hanes & TapeLift & 26 & 7 & 78 \\
\hline Hanes & TapeLift & 26 & 8 & 70 \\
\hline Hanes & TapeLift & 26 & 9 & 69 \\
\hline Hanes & TapeLift & 26 & 10 & 96 \\
\hline Hanes & TapeLift & 26 & 11 & 90 \\
\hline
\end{tabular}




\begin{tabular}{|c|c|c|c|c|}
\hline Hanes & TapeLift & 26 & 12 & 55 \\
\hline Hanes & TapeLift & 26 & 13 & 81 \\
\hline Hanes & TapeLift & 26 & 14 & 90 \\
\hline Hanes & TapeLift & 26 & 15 & 92 \\
\hline Hanes & TapeLift & 27 & 1 & 82 \\
\hline Hanes & TapeLift & 27 & 2 & 65 \\
\hline Hanes & TapeLift & 27 & 3 & 65 \\
\hline Hanes & TapeLift & 27 & 4 & 71 \\
\hline Hanes & TapeLift & 27 & 5 & 76 \\
\hline Hanes & TapeLift & 27 & 6 & 76 \\
\hline Hanes & TapeLift & 27 & 7 & 66 \\
\hline Hanes & TapeLift & 27 & 8 & 74 \\
\hline Hanes & TapeLift & 27 & 9 & 56 \\
\hline Hanes & TapeLift & 27 & 10 & 37 \\
\hline Hanes & TapeLift & 27 & 11 & 74 \\
\hline Hanes & TapeLift & 27 & 12 & 43 \\
\hline Hanes & TapeLift & 27 & 13 & 52 \\
\hline Hanes & TapeLift & 27 & 14 & 99 \\
\hline Hanes & TapeLift & 27 & 15 & 83 \\
\hline Hanes & TapeLift & 27 & 16 & 54 \\
\hline Hanes & TapeLift & 27 & 17 & 52 \\
\hline Hanes & TapeLift & 27 & 18 & 51 \\
\hline Hanes & TapeLift & 27 & 19 & 38 \\
\hline Hanes & TapeLift & 27 & 20 & 44 \\
\hline Hanes & TapeLift & 28 & 1 & 63 \\
\hline Hanes & TapeLift & 28 & 2 & 44 \\
\hline Hanes & TapeLift & 28 & 3 & 51 \\
\hline Hanes & TapeLift & 28 & 4 & 54 \\
\hline Hanes & TapeLift & 28 & 5 & 44 \\
\hline Hanes & TapeLift & 28 & 6 & 78 \\
\hline Hanes & TapeLift & 28 & 7 & 101 \\
\hline Hanes & TapeLift & 28 & 8 & 20 \\
\hline Hanes & TapeLift & 28 & 9 & 74 \\
\hline Hanes & TapeLift & 28 & 10 & 88 \\
\hline Hanes & TapeLift & 28 & 11 & 70 \\
\hline Hanes & TapeLift & 28 & 12 & 37 \\
\hline Hanes & TapeLift & 28 & 13 & 41 \\
\hline Hanes & TapeLift & 28 & 14 & 46 \\
\hline Hanes & TapeLift & 28 & 15 & 54 \\
\hline Hanes & TapeLift & 29 & 1 & 38 \\
\hline Hanes & TapeLift & 29 & 2 & 66 \\
\hline Hanes & TapeLift & 29 & 3 & 90 \\
\hline Hanes & TapeLift & 29 & 4 & 90 \\
\hline Hanes & TapeLift & 29 & 5 & 87 \\
\hline Hanes & TapeLift & 29 & 6 & 76 \\
\hline
\end{tabular}




\begin{tabular}{|c|c|c|c|c|}
\hline Hanes & TapeLift & 29 & 7 & 65 \\
\hline Hanes & TapeLift & 29 & 8 & 104 \\
\hline Hanes & TapeLift & 29 & 9 & 108 \\
\hline Hanes & TapeLift & 29 & 10 & 93 \\
\hline Hanes & TapeLift & 29 & 11 & 72 \\
\hline Hanes & TapeLift & 29 & 12 & 49 \\
\hline Hanes & TapeLift & 29 & 13 & 38 \\
\hline Hanes & TapeLift & 29 & 14 & 65 \\
\hline Hanes & TapeLift & 29 & 15 & 82 \\
\hline Hanes & TapeLift & 29 & 16 & 62 \\
\hline Hanes & TapeLift & 29 & 17 & 84 \\
\hline Hanes & TapeLift & 29 & 18 & 10 \\
\hline Hanes & TapeLift & 29 & 19 & 62 \\
\hline Hanes & TapeLift & 29 & 20 & 60 \\
\hline Hanes & TapeLift & 30 & 1 & 77 \\
\hline Hanes & TapeLift & 30 & 2 & 57 \\
\hline Hanes & TapeLift & 30 & 3 & 75 \\
\hline Hanes & TapeLift & 30 & 4 & 112 \\
\hline Hanes & TapeLift & 30 & 5 & 110 \\
\hline Hanes & TapeLift & 30 & 6 & 70 \\
\hline Hanes & TapeLift & 30 & 7 & 121 \\
\hline Hanes & TapeLift & 30 & 8 & 66 \\
\hline Hanes & TapeLift & 30 & 9 & 40 \\
\hline Hanes & TapeLift & 30 & 10 & 77 \\
\hline Hanes & TapeLift & 30 & 11 & 84 \\
\hline Hanes & TapeLift & 30 & 12 & 87 \\
\hline Hanes & TapeLift & 30 & 13 & 108 \\
\hline Hanes & TapeLift & 30 & 14 & 95 \\
\hline Hanes & TapeLift & 30 & 15 & 83 \\
\hline Hanes & TapeLift & 31 & 1 & 133 \\
\hline Hanes & TapeLift & 31 & 2 & 41 \\
\hline Hanes & TapeLift & 31 & 3 & 73 \\
\hline Hanes & TapeLift & 31 & 4 & 54 \\
\hline Hanes & TapeLift & 31 & 5 & 49 \\
\hline Hanes & TapeLift & 31 & 6 & 100 \\
\hline Hanes & TapeLift & 31 & 7 & 66 \\
\hline Hanes & TapeLift & 31 & 8 & 47 \\
\hline Hanes & TapeLift & 31 & 9 & 92 \\
\hline Hanes & TapeLift & 31 & 10 & 121 \\
\hline Hanes & TapeLift & 31 & 11 & 73 \\
\hline Hanes & TapeLift & 31 & 12 & 62 \\
\hline Hanes & TapeLift & 31 & 13 & 101 \\
\hline Hanes & TapeLift & 31 & 14 & 66 \\
\hline Hanes & TapeLift & 31 & 15 & 67 \\
\hline Hanes & TapeLift & 31 & 16 & 61 \\
\hline
\end{tabular}




\begin{tabular}{|c|c|c|c|c|}
\hline Hanes & TapeLift & 31 & 17 & 75 \\
\hline Hanes & TapeLift & 32 & 1 & 72 \\
\hline Hanes & TapeLift & 32 & 2 & 47 \\
\hline Hanes & TapeLift & 32 & 3 & 51 \\
\hline Hanes & TapeLift & 32 & 4 & 37 \\
\hline Hanes & TapeLift & 32 & 5 & 61 \\
\hline Hanes & TapeLift & 32 & 6 & 63 \\
\hline Hanes & TapeLift & 32 & 7 & 43 \\
\hline Hanes & TapeLift & 32 & 8 & 35 \\
\hline Hanes & TapeLift & 32 & 9 & 50 \\
\hline Hanes & TapeLift & 32 & 10 & 69 \\
\hline Hanes & TapeLift & 32 & 11 & 20 \\
\hline Hanes & TapeLift & 32 & 12 & 55 \\
\hline Hanes & TapeLift & 32 & 13 & 58 \\
\hline Hanes & TapeLift & 33 & 1 & 39 \\
\hline Hanes & TapeLift & 33 & 2 & 35 \\
\hline Hanes & TapeLift & 33 & 3 & 60 \\
\hline Hanes & TapeLift & 33 & 4 & 118 \\
\hline Hanes & TapeLift & 33 & 5 & 81 \\
\hline Hanes & TapeLift & 34 & 1 & 71 \\
\hline Hanes & TapeLift & 34 & 2 & 55 \\
\hline Hanes & TapeLift & 34 & 3 & 68 \\
\hline Hanes & TapeLift & 34 & 4 & 60 \\
\hline Hanes & TapeLift & 34 & 5 & 48 \\
\hline Hanes & TapeLift & 35 & 1 & 49 \\
\hline Hanes & TapeLift & 35 & 2 & 74 \\
\hline Hanes & TapeLift & 35 & 3 & 63 \\
\hline Hanes & TapeLift & 35 & 4 & 41 \\
\hline Hanes & Vacuum & 1 & 1 & 61 \\
\hline Hanes & Vacuum & 1 & 2 & 1 \\
\hline Hanes & Vacuum & 1 & 3 & 61 \\
\hline Hanes & Vacuum & 1 & 4 & 4 \\
\hline Hanes & Vacuum & 1 & 5 & 79 \\
\hline Hanes & Vacuum & 2 & 1 & 7 \\
\hline Hanes & Vacuum & 2 & 2 & 82 \\
\hline Hanes & Vacuum & 2 & 3 & 4 \\
\hline Hanes & Vacuum & 2 & 4 & 70 \\
\hline Hanes & Vacuum & 2 & 5 & 8 \\
\hline Hanes & Vacuum & 2 & 6 & 109 \\
\hline Hanes & Vacuum & 2 & 7 & 4 \\
\hline Hanes & Vacuum & 2 & 8 & 87 \\
\hline Hanes & Vacuum & 3 & 1 & 2 \\
\hline Hanes & Vacuum & 3 & 2 & 78 \\
\hline Hanes & Vacuum & 3 & 3 & 3 \\
\hline Hanes & Vacuum & 3 & 4 & 73 \\
\hline
\end{tabular}




\begin{tabular}{|c|c|c|c|c|}
\hline Hanes & Vacuum & 3 & 5 & 7 \\
\hline Hanes & Vacuum & 5 & 1 & 102 \\
\hline Hanes & Vacuum & 5 & 2 & 8 \\
\hline Hanes & Vacuum & 5 & 3 & 3 \\
\hline Hanes & Vacuum & 5 & 4 & 4 \\
\hline Hanes & Vacuum & 6 & 1 & 5 \\
\hline Hanes & Vacuum & 6 & 2 & 4 \\
\hline Hanes & Vacuum & 6 & 3 & 88 \\
\hline Hanes & Vacuum & 6 & 4 & 7 \\
\hline Hanes & Vacuum & 6 & 5 & 97 \\
\hline Hanes & Vacuum & 6 & 6 & 6 \\
\hline Hanes & Vacuum & 7 & 1 & 3 \\
\hline Hanes & Vacuum & 7 & 2 & 4 \\
\hline Hanes & Vacuum & 7 & 3 & 109 \\
\hline Hanes & Vacuum & 7 & 4 & 4 \\
\hline Hanes & Vacuum & 8 & 1 & 66 \\
\hline Hanes & Vacuum & 8 & 2 & 4 \\
\hline Hanes & Vacuum & 8 & 3 & 136 \\
\hline Hanes & Vacuum & 8 & 4 & 2 \\
\hline Hanes & Vacuum & 9 & 1 & 55 \\
\hline Hanes & Vacuum & 9 & 2 & 3 \\
\hline Hanes & Vacuum & 9 & 3 & 96 \\
\hline Hanes & Vacuum & 9 & 4 & 3 \\
\hline Hanes & Vacuum & 9 & 5 & 28 \\
\hline Hanes & Vacuum & 9 & 6 & 4 \\
\hline Hanes & Vacuum & 10 & 1 & 22 \\
\hline Hanes & Vacuum & 10 & 2 & 4 \\
\hline Hanes & Vacuum & 10 & 3 & 75 \\
\hline Hanes & Vacuum & 10 & 4 & 8 \\
\hline Hanes & Vacuum & 10 & 5 & 92 \\
\hline Hanes & Vacuum & 11 & 1 & 4 \\
\hline Hanes & Vacuum & 11 & 2 & 51 \\
\hline Hanes & Vacuum & 11 & 3 & 6 \\
\hline Hanes & Vacuum & 11 & 4 & 68 \\
\hline Hanes & Vacuum & 11 & 5 & 7 \\
\hline Hanes & Vacuum & 11 & 6 & 66 \\
\hline Hanes & Vacuum & 11 & 7 & 1 \\
\hline Hanes & Vacuum & 12 & 1 & 68 \\
\hline Hanes & Vacuum & 12 & 2 & 2 \\
\hline Hanes & Vacuum & 13 & 1 & 61 \\
\hline Hanes & Vacuum & 13 & 2 & 2 \\
\hline Hanes & Vacuum & 13 & 3 & 92 \\
\hline Hanes & Vacuum & 13 & 4 & 4 \\
\hline Hanes & Vacuum & 13 & 5 & 35 \\
\hline Hanes & Vacuum & 13 & 6 & 5 \\
\hline
\end{tabular}




\begin{tabular}{|c|c|c|c|c|}
\hline Hanes & Vacuum & 13 & 7 & 86 \\
\hline Hanes & Vacuum & 14 & 1 & 4 \\
\hline Hanes & Vacuum & 14 & 2 & 39 \\
\hline Hanes & Vacuum & 14 & 3 & 4 \\
\hline Hanes & Vacuum & 14 & 4 & 137 \\
\hline Hanes & Vacuum & 14 & 5 & 9 \\
\hline Hanes & Vacuum & 14 & 6 & 91 \\
\hline Hanes & Vacuum & 14 & 7 & 3 \\
\hline Hanes & Vacuum & 14 & 8 & 126 \\
\hline Hanes & Vacuum & 15 & 1 & 4 \\
\hline Hanes & Vacuum & 15 & 2 & 112 \\
\hline Hanes & Vacuum & 15 & 3 & 4 \\
\hline Hanes & Vacuum & 15 & 4 & 26 \\
\hline Hanes & Vacuum & 15 & 5 & 4 \\
\hline Hanes & Vacuum & 16 & 1 & 25 \\
\hline Hanes & Vacuum & 16 & 2 & 4 \\
\hline Hanes & Vacuum & 16 & 3 & 101 \\
\hline Hanes & Vacuum & 16 & 4 & 3 \\
\hline Hanes & Vacuum & 16 & 5 & 83 \\
\hline Hanes & Vacuum & 17 & 1 & 1 \\
\hline Hanes & Vacuum & 17 & 2 & 88 \\
\hline Hanes & Vacuum & 17 & 3 & 3 \\
\hline Hanes & Vacuum & 17 & 4 & 61 \\
\hline Hanes & Vacuum & 17 & 5 & 1 \\
\hline Hanes & Vacuum & 17 & 6 & 81 \\
\hline Hanes & Vacuum & 17 & 7 & 3 \\
\hline Hanes & Vacuum & 17 & 8 & 75 \\
\hline Hanes & Vacuum & 17 & 9 & 3 \\
\hline Hanes & Vacuum & 17 & 10 & 63 \\
\hline Hanes & Vacuum & 18 & 1 & 2 \\
\hline Hanes & Vacuum & 18 & 2 & 39 \\
\hline Hanes & Vacuum & 18 & 3 & 4 \\
\hline Hanes & Vacuum & 18 & 4 & 75 \\
\hline Hanes & Vacuum & 18 & 5 & 6 \\
\hline Hanes & Vacuum & 18 & 6 & 59 \\
\hline Hanes & Vacuum & 18 & 7 & 2 \\
\hline Hanes & Vacuum & 18 & 8 & 41 \\
\hline Hanes & Vacuum & 19 & 1 & 3 \\
\hline Hanes & Vacuum & 19 & 2 & 98 \\
\hline Hanes & Vacuum & 19 & 3 & 4 \\
\hline Hanes & Vacuum & 19 & 4 & 121 \\
\hline Hanes & Vacuum & 19 & 5 & 8 \\
\hline Hanes & Vacuum & 19 & 6 & 67 \\
\hline Hanes & Vacuum & 19 & 7 & 4 \\
\hline Hanes & Vacuum & 19 & 8 & 83 \\
\hline
\end{tabular}




\begin{tabular}{|c|c|c|c|c|}
\hline Hanes & Vacuum & 19 & 9 & 2 \\
\hline Hanes & Vacuum & 19 & 10 & 85 \\
\hline Hanes & Vacuum & 19 & 11 & 3 \\
\hline Hanes & Vacuum & 19 & 12 & 82 \\
\hline Hanes & Vacuum & 19 & 13 & 107 \\
\hline Hanes & Vacuum & 19 & 14 & 48 \\
\hline Hanes & Vacuum & 20 & 1 & 83 \\
\hline Hanes & Vacuum & 20 & 2 & 73 \\
\hline Hanes & Vacuum & 20 & 3 & 23 \\
\hline Hanes & Vacuum & 20 & 4 & 104 \\
\hline Hanes & Vacuum & 20 & 5 & 94 \\
\hline Hanes & Vacuum & 20 & 6 & 110 \\
\hline Hanes & Vacuum & 20 & 7 & 14 \\
\hline Hanes & Vacuum & 20 & 8 & 24 \\
\hline Hanes & Vacuum & 20 & 9 & 107 \\
\hline Hanes & Vacuum & 21 & 1 & 99 \\
\hline Hanes & Vacuum & 21 & 2 & 96 \\
\hline Hanes & Vacuum & 21 & 3 & 96 \\
\hline Hanes & Vacuum & 21 & 4 & 147 \\
\hline Hanes & Vacuum & 21 & 5 & 126 \\
\hline Hanes & Vacuum & 21 & 6 & 14 \\
\hline Hanes & Vacuum & 21 & 7 & 67 \\
\hline Hanes & Vacuum & 21 & 8 & 43 \\
\hline Hanes & Vacuum & 21 & 9 & 61 \\
\hline Hanes & Vacuum & 21 & 10 & 63 \\
\hline Hanes & Vacuum & 21 & 11 & 154 \\
\hline Hanes & Vacuum & 21 & 12 & 3 \\
\hline Hanes & Vacuum & 22 & 1 & 73 \\
\hline Hanes & Vacuum & 22 & 2 & 4 \\
\hline Hanes & Vacuum & 22 & 3 & 58 \\
\hline Hanes & Vacuum & 22 & 4 & 6 \\
\hline Hanes & Vacuum & 22 & 5 & 103 \\
\hline Hanes & Vacuum & 22 & 6 & 3 \\
\hline Hanes & Vacuum & 22 & 7 & 153 \\
\hline Hanes & Vacuum & 22 & 8 & 5 \\
\hline Hanes & Vacuum & 22 & 9 & 75 \\
\hline Hanes & Vacuum & 22 & 10 & 4 \\
\hline Hanes & Vacuum & 23 & 1 & 60 \\
\hline Hanes & Vacuum & 23 & 2 & 4 \\
\hline Hanes & Vacuum & 23 & 3 & 103 \\
\hline Hanes & Vacuum & 23 & 4 & 8 \\
\hline Hanes & Vacuum & 23 & 5 & 31 \\
\hline Hanes & Vacuum & 23 & 6 & 38 \\
\hline Hanes & Vacuum & 23 & 7 & 51 \\
\hline Hanes & Vacuum & 23 & 8 & 106 \\
\hline
\end{tabular}




\begin{tabular}{|c|c|}
\hline Hanes & Vacuum \\
\hline Hanes & Vacuum \\
\hline Hanes & Vacuum \\
\hline Hanes & Vacuum \\
\hline Hanes & Vacuum \\
\hline Hanes & Vacuum \\
\hline Hanes & Vacuum \\
\hline Hanes & Vacuum \\
\hline Hanes & Vacuum \\
\hline Hanes & Vacuum \\
\hline Hanes & Vacuum \\
\hline Hanes & Vacuum \\
\hline Hanes & Vacuum \\
\hline Hanes & Vacuum \\
\hline Hanes & Vacuum \\
\hline Hanes & Vacuum \\
\hline Hanes & Vacuum \\
\hline Hanes & Vacuum \\
\hline Hanes & Vacuum \\
\hline Hanes & Vacuum \\
\hline Hanes & Vacuum \\
\hline Hanes & Vacuum \\
\hline Hanes & Vacuum \\
\hline Hanes & Vacuum \\
\hline Hanes & Vacuum \\
\hline Hanes & Vacuum \\
\hline Hanes & Vacuum \\
\hline Hanes & Vacuum \\
\hline Hanes & Vacuum \\
\hline Hanes & Vacuum \\
\hline Hanes & Vacuum \\
\hline Hanes & Vacuum \\
\hline Hanes & Vacuum \\
\hline Hanes & Vacuum \\
\hline Hanes & Vacuum \\
\hline Hanes & Vacuum \\
\hline Hanes & Vacuum \\
\hline Hanes & Vacuum \\
\hline Hanes & Vacuum \\
\hline Hanes & Vacuum \\
\hline Hanes & Vacuum \\
\hline Hanes & Vacuum \\
\hline Hanes & Vacuum \\
\hline Hanes & Vacuum \\
\hline Hanes & Vacuum \\
\hline
\end{tabular}

\begin{tabular}{|c|c|c|}
\hline 23 & 9 & 38 \\
\hline 23 & 10 & 128 \\
\hline 24 & 1 & 81 \\
\hline 24 & 2 & 13 \\
\hline 24 & 3 & 122 \\
\hline 24 & 4 & 50 \\
\hline 24 & 5 & 101 \\
\hline 24 & 6 & 114 \\
\hline 24 & 7 & 127 \\
\hline 24 & 8 & 68 \\
\hline 24 & 9 & 163 \\
\hline 24 & 10 & 96 \\
\hline 25 & 1 & 92 \\
\hline 25 & 2 & 137 \\
\hline 25 & 3 & 44 \\
\hline 25 & 4 & 22 \\
\hline 25 & 5 & 29 \\
\hline 25 & 6 & 59 \\
\hline 25 & 7 & 126 \\
\hline 25 & 8 & 6 \\
\hline 25 & 9 & 26 \\
\hline 25 & 10 & 96 \\
\hline 26 & 1 & 124 \\
\hline 26 & 2 & 104 \\
\hline 26 & 3 & 119 \\
\hline 26 & 4 & 124 \\
\hline 26 & 5 & 115 \\
\hline 26 & 6 & 87 \\
\hline 26 & 7 & 133 \\
\hline 26 & 8 & 46 \\
\hline 27 & 1 & 32 \\
\hline 27 & 2 & 90 \\
\hline 27 & 3 & 86 \\
\hline 27 & 4 & 80 \\
\hline 27 & 5 & 81 \\
\hline 27 & 6 & 80 \\
\hline 27 & 7 & 70 \\
\hline 27 & 8 & 55 \\
\hline 27 & 9 & 5 \\
\hline 27 & 10 & 97 \\
\hline 27 & 11 & 120 \\
\hline 27 & 12 & 101 \\
\hline 27 & 13 & 11 \\
\hline 27 & 14 & 12 \\
\hline 28 & 1 & 11 \\
\hline
\end{tabular}




\begin{tabular}{|c|c|c|c|c|}
\hline Hanes & Vacuum & 28 & 2 & 11 \\
\hline Hanes & Vacuum & 28 & 3 & 9 \\
\hline Hanes & Vacuum & 28 & 4 & 1 \\
\hline Hanes & Vacuum & 28 & 5 & 71 \\
\hline Hanes & Vacuum & 28 & 6 & 100 \\
\hline Hanes & Vacuum & 28 & 7 & 94 \\
\hline Hanes & Vacuum & 29 & 1 & 10 \\
\hline Hanes & Vacuum & 29 & 2 & 60 \\
\hline Hanes & Vacuum & 29 & 3 & 44 \\
\hline Hanes & Vacuum & 29 & 4 & 104 \\
\hline Hanes & Vacuum & 29 & 5 & 32 \\
\hline Hanes & Vacuum & 29 & 6 & 63 \\
\hline Hanes & Vacuum & 29 & 7 & 152 \\
\hline Hanes & Vacuum & 29 & 8 & 25 \\
\hline Hanes & Vacuum & 29 & 9 & 86 \\
\hline Hanes & Vacuum & 29 & 10 & 6 \\
\hline Hanes & Vacuum & 29 & 11 & 74 \\
\hline Hanes & Vacuum & 29 & 12 & 46 \\
\hline Hanes & Vacuum & 30 & 1 & 122 \\
\hline Hanes & Vacuum & 30 & 2 & 117 \\
\hline Hanes & Vacuum & 30 & 3 & 33 \\
\hline Hanes & Vacuum & 31 & 1 & 38 \\
\hline Hanes & Vacuum & 31 & 2 & 25 \\
\hline Hanes & Vacuum & 31 & 3 & 104 \\
\hline Hanes & Vacuum & 31 & 4 & 25 \\
\hline Hanes & Vacuum & 31 & 5 & 111 \\
\hline Hanes & Vacuum & 31 & 6 & 26 \\
\hline Hanes & Vacuum & 31 & 7 & 17 \\
\hline Hanes & Vacuum & 31 & 8 & 32 \\
\hline Hanes & Vacuum & 32 & 1 & 28 \\
\hline Hanes & Vacuum & 32 & 2 & 25 \\
\hline Hanes & Vacuum & 32 & 3 & 98 \\
\hline Hanes & Vacuum & 32 & 4 & 24 \\
\hline Hanes & Vacuum & 32 & 5 & 40 \\
\hline Hanes & Vacuum & 32 & 6 & 91 \\
\hline Hanes & Vacuum & 32 & 7 & 102 \\
\hline Hanes & Vacuum & 32 & 8 & 106 \\
\hline Hanes & Vacuum & 32 & 9 & 81 \\
\hline Hanes & Vacuum & 32 & 10 & 92 \\
\hline Hanes & Vacuum & 32 & 11 & 61 \\
\hline Hanes & Vacuum & 32 & 12 & 26 \\
\hline Hanes & Vacuum & 33 & 1 & 90 \\
\hline Hanes & Vacuum & 33 & 2 & 30 \\
\hline Hanes & Vacuum & 33 & 3 & 9 \\
\hline Hanes & Vacuum & 33 & 4 & 89 \\
\hline
\end{tabular}




\begin{tabular}{|c|c|c|c|c|}
\hline Hanes & Vacuum & 33 & 5 & 10 \\
\hline Hanes & Vacuum & 33 & 6 & 32 \\
\hline Hanes & Vacuum & 33 & 7 & 101 \\
\hline Hanes & Vacuum & 33 & 8 & 41 \\
\hline Hanes & Vacuum & 33 & 9 & 107 \\
\hline Hanes & Vacuum & 33 & 10 & 28 \\
\hline Hanes & Vacuum & 34 & 1 & 76 \\
\hline Hanes & Vacuum & 34 & 2 & 11 \\
\hline Hanes & Vacuum & 34 & 3 & 88 \\
\hline Hanes & Vacuum & 34 & 4 & 10 \\
\hline Hanes & Vacuum & 34 & 5 & 111 \\
\hline Hanes & Vacuum & 35 & 1 & 4 \\
\hline Hanes & Vacuum & 35 & 2 & 53 \\
\hline Hanes & Vacuum & 35 & 3 & 5 \\
\hline Hanes & Vacuum & 35 & 4 & 81 \\
\hline Hanes & Vacuum & 35 & 5 & 7 \\
\hline Hanes & Vacuum & 35 & 6 & 102 \\
\hline Nylons & TapeLift & 1 & 1 & 79 \\
\hline Nylons & TapeLift & 1 & 2 & 50 \\
\hline Nylons & TapeLift & 1 & 3 & 25 \\
\hline Nylons & TapeLift & 1 & 4 & 40 \\
\hline Nylons & TapeLift & 1 & 5 & 64 \\
\hline Nylons & TapeLift & 1 & 6 & 65 \\
\hline Nylons & TapeLift & 1 & 7 & 73 \\
\hline Nylons & TapeLift & 1 & 8 & 90 \\
\hline Nylons & TapeLift & 1 & 9 & 45 \\
\hline Nylons & TapeLift & 1 & 10 & 78 \\
\hline Nylons & TapeLift & 1 & 11 & 72 \\
\hline Nylons & TapeLift & 1 & 12 & 40 \\
\hline Nylons & TapeLift & 1 & 13 & 56 \\
\hline Nylons & TapeLift & 1 & 14 & 56 \\
\hline Nylons & TapeLift & 1 & 15 & 69 \\
\hline Nylons & TapeLift & 1 & 16 & 77 \\
\hline Nylons & TapeLift & 1 & 17 & 110 \\
\hline Nylons & TapeLift & 1 & 18 & 54 \\
\hline Nylons & TapeLift & 1 & 19 & 38 \\
\hline Nylons & TapeLift & 1 & 20 & 62 \\
\hline Nylons & TapeLift & 2 & 1 & 81 \\
\hline Nylons & TapeLift & 2 & 2 & 48 \\
\hline Nylons & TapeLift & 2 & 3 & 46 \\
\hline Nylons & TapeLift & 2 & 4 & 63 \\
\hline Nylons & TapeLift & 2 & 5 & 58 \\
\hline Nylons & TapeLift & 2 & 6 & 47 \\
\hline Nylons & TapeLift & 2 & 7 & 48 \\
\hline Nylons & TapeLift & 2 & 8 & 63 \\
\hline
\end{tabular}




\begin{tabular}{|c|c|c|c|c|}
\hline Nylons & TapeLift & 2 & 9 & 72 \\
\hline Nylons & TapeLift & 2 & 10 & 57 \\
\hline Nylons & TapeLift & 2 & 11 & 45 \\
\hline Nylons & TapeLift & 2 & 12 & 68 \\
\hline Nylons & TapeLift & 2 & 13 & 73 \\
\hline Nylons & TapeLift & 2 & 14 & 106 \\
\hline Nylons & TapeLift & 2 & 15 & 72 \\
\hline Nylons & TapeLift & 2 & 16 & 66 \\
\hline Nylons & TapeLift & 2 & 17 & 124 \\
\hline Nylons & TapeLift & 2 & 18 & 87 \\
\hline Nylons & TapeLift & 2 & 19 & 77 \\
\hline Nylons & TapeLift & 2 & 20 & 54 \\
\hline Nylons & TapeLift & 3 & 1 & 81 \\
\hline Nylons & TapeLift & 3 & 2 & 56 \\
\hline Nylons & TapeLift & 3 & 3 & 82 \\
\hline Nylons & TapeLift & 3 & 4 & 51 \\
\hline Nylons & TapeLift & 3 & 5 & 62 \\
\hline Nylons & TapeLift & 3 & 6 & 61 \\
\hline Nylons & TapeLift & 3 & 7 & 106 \\
\hline Nylons & TapeLift & 3 & 8 & 91 \\
\hline Nylons & TapeLift & 3 & 9 & 76 \\
\hline Nylons & TapeLift & 3 & 10 & 97 \\
\hline Nylons & TapeLift & 3 & 11 & 57 \\
\hline Nylons & TapeLift & 3 & 12 & 51 \\
\hline Nylons & TapeLift & 3 & 13 & 60 \\
\hline Nylons & TapeLift & 3 & 14 & 35 \\
\hline Nylons & TapeLift & 3 & 15 & 57 \\
\hline Nylons & TapeLift & 3 & 16 & 92 \\
\hline Nylons & TapeLift & 3 & 17 & 116 \\
\hline Nylons & TapeLift & 3 & 18 & 106 \\
\hline Nylons & TapeLift & 3 & 19 & 69 \\
\hline Nylons & TapeLift & 3 & 20 & 72 \\
\hline Nylons & TapeLift & 4 & 1 & 82 \\
\hline Nylons & TapeLift & 4 & 2 & 74 \\
\hline Nylons & TapeLift & 4 & 3 & 93 \\
\hline Nylons & TapeLift & 4 & 4 & 73 \\
\hline Nylons & TapeLift & 4 & 5 & 62 \\
\hline Nylons & TapeLift & 4 & 6 & 49 \\
\hline Nylons & TapeLift & 4 & 7 & 72 \\
\hline Nylons & TapeLift & 4 & 8 & 82 \\
\hline Nylons & TapeLift & 4 & 9 & 74 \\
\hline Nylons & TapeLift & 4 & 10 & 50 \\
\hline Nylons & TapeLift & 4 & 11 & 100 \\
\hline Nylons & TapeLift & 4 & 12 & 93 \\
\hline Nylons & TapeLift & 4 & 13 & 79 \\
\hline
\end{tabular}




\begin{tabular}{|c|c|c|c|c|}
\hline Nylons & TapeLift & 4 & 14 & 112 \\
\hline Nylons & TapeLift & 4 & 15 & 93 \\
\hline Nylons & TapeLift & 4 & 16 & 47 \\
\hline Nylons & TapeLift & 4 & 17 & 41 \\
\hline Nylons & TapeLift & 4 & 18 & 62 \\
\hline Nylons & TapeLift & 4 & 19 & 75 \\
\hline Nylons & TapeLift & 4 & 20 & 38 \\
\hline Nylons & TapeLift & 5 & 1 & 66 \\
\hline Nylons & TapeLift & 5 & 2 & 65 \\
\hline Nylons & TapeLift & 5 & 3 & 22 \\
\hline Nylons & TapeLift & 5 & 4 & 50 \\
\hline Nylons & TapeLift & 5 & 5 & 63 \\
\hline Nylons & TapeLift & 5 & 6 & 91 \\
\hline Nylons & TapeLift & 5 & 7 & 46 \\
\hline Nylons & TapeLift & 5 & 8 & 38 \\
\hline Nylons & TapeLift & 5 & 9 & 57 \\
\hline Nylons & TapeLift & 5 & 10 & 52 \\
\hline Nylons & TapeLift & 5 & 11 & 32 \\
\hline Nylons & TapeLift & 5 & 12 & 7 \\
\hline Nylons & TapeLift & 5 & 13 & 8 \\
\hline Nylons & TapeLift & 5 & 14 & 4 \\
\hline Nylons & TapeLift & 5 & 15 & 15 \\
\hline Nylons & TapeLift & 5 & 16 & 19 \\
\hline Nylons & TapeLift & 5 & 17 & 18 \\
\hline Nylons & TapeLift & 5 & 18 & 13 \\
\hline Nylons & TapeLift & 5 & 19 & 71 \\
\hline Nylons & TapeLift & 5 & 20 & 27 \\
\hline Nylons & TapeLift & 6 & 1 & 11 \\
\hline Nylons & TapeLift & 6 & 2 & 21 \\
\hline Nylons & TapeLift & 6 & 3 & 7 \\
\hline Nylons & TapeLift & 6 & 4 & 30 \\
\hline Nylons & TapeLift & 6 & 5 & 16 \\
\hline Nylons & TapeLift & 6 & 6 & 85 \\
\hline Nylons & TapeLift & 6 & 7 & 72 \\
\hline Nylons & TapeLift & 6 & 8 & 89 \\
\hline Nylons & TapeLift & 6 & 9 & 124 \\
\hline Nylons & TapeLift & 6 & 10 & 76 \\
\hline Nylons & TapeLift & 6 & 11 & 95 \\
\hline Nylons & TapeLift & 6 & 12 & 67 \\
\hline Nylons & TapeLift & 6 & 13 & 75 \\
\hline Nylons & TapeLift & 6 & 14 & 30 \\
\hline Nylons & TapeLift & 6 & 15 & 71 \\
\hline Nylons & TapeLift & 6 & 16 & 94 \\
\hline Nylons & TapeLift & 6 & 17 & 80 \\
\hline Nylons & TapeLift & 6 & 18 & 57 \\
\hline
\end{tabular}




\begin{tabular}{|c|c|c|c|c|}
\hline Nylons & TapeLift & 6 & 19 & 45 \\
\hline Nylons & TapeLift & 6 & 20 & 40 \\
\hline Nylons & TapeLift & 7 & 1 & 56 \\
\hline Nylons & TapeLift & 7 & 2 & 108 \\
\hline Nylons & TapeLift & 7 & 3 & 93 \\
\hline Nylons & TapeLift & 7 & 4 & 68 \\
\hline Nylons & TapeLift & 7 & 5 & 87 \\
\hline Nylons & TapeLift & 7 & 6 & 69 \\
\hline Nylons & TapeLift & 7 & 7 & 143 \\
\hline Nylons & TapeLift & 7 & 8 & 33 \\
\hline Nylons & TapeLift & 7 & 9 & 17 \\
\hline Nylons & TapeLift & 7 & 10 & 30 \\
\hline Nylons & TapeLift & 7 & 11 & 81 \\
\hline Nylons & TapeLift & 7 & 12 & 23 \\
\hline Nylons & TapeLift & 7 & 13 & 63 \\
\hline Nylons & TapeLift & 7 & 14 & 49 \\
\hline Nylons & TapeLift & 7 & 15 & 25 \\
\hline Nylons & TapeLift & 7 & 16 & 28 \\
\hline Nylons & TapeLift & 7 & 17 & 38 \\
\hline Nylons & TapeLift & 7 & 18 & 29 \\
\hline Nylons & TapeLift & 7 & 19 & 21 \\
\hline Nylons & TapeLift & 7 & 20 & 53 \\
\hline Nylons & TapeLift & 7 & 21 & 66 \\
\hline Nylons & TapeLift & 7 & 22 & 58 \\
\hline Nylons & TapeLift & 8 & 1 & 20 \\
\hline Nylons & TapeLift & 8 & 2 & 159 \\
\hline Nylons & TapeLift & 8 & 3 & 96 \\
\hline Nylons & TapeLift & 8 & 4 & 14 \\
\hline Nylons & TapeLift & 8 & 5 & 43 \\
\hline Nylons & TapeLift & 8 & 6 & 25 \\
\hline Nylons & TapeLift & 8 & 7 & 79 \\
\hline Nylons & TapeLift & 8 & 8 & 19 \\
\hline Nylons & TapeLift & 8 & 9 & 31 \\
\hline Nylons & TapeLift & 8 & 10 & 21 \\
\hline Nylons & TapeLift & 8 & 11 & 39 \\
\hline Nylons & TapeLift & 8 & 12 & 79 \\
\hline Nylons & TapeLift & 8 & 13 & 16 \\
\hline Nylons & TapeLift & 8 & 14 & 56 \\
\hline Nylons & TapeLift & 8 & 15 & 30 \\
\hline Nylons & TapeLift & 8 & 16 & 10 \\
\hline Nylons & TapeLift & 8 & 17 & 22 \\
\hline Nylons & TapeLift & 8 & 18 & 24 \\
\hline Nylons & TapeLift & 8 & 19 & 52 \\
\hline Nylons & TapeLift & 8 & 20 & 69 \\
\hline Nylons & TapeLift & 8 & 21 & 34 \\
\hline
\end{tabular}




\begin{tabular}{|c|c|c|c|c|}
\hline Nylons & TapeLift & 8 & 22 & 52 \\
\hline Nylons & TapeLift & 8 & 23 & 32 \\
\hline Nylons & TapeLift & 8 & 24 & 98 \\
\hline Nylons & TapeLift & 8 & 25 & 74 \\
\hline Nylons & TapeLift & 9 & 1 & 77 \\
\hline Nylons & TapeLift & 9 & 2 & 64 \\
\hline Nylons & TapeLift & 9 & 3 & 257 \\
\hline Nylons & TapeLift & 9 & 4 & 60 \\
\hline Nylons & TapeLift & 9 & 5 & 86 \\
\hline Nylons & TapeLift & 9 & 6 & 65 \\
\hline Nylons & TapeLift & 9 & 7 & 19 \\
\hline Nylons & TapeLift & 9 & 8 & 41 \\
\hline Nylons & TapeLift & 9 & 9 & 34 \\
\hline Nylons & TapeLift & 9 & 10 & 12 \\
\hline Nylons & TapeLift & 9 & 11 & 19 \\
\hline Nylons & TapeLift & 9 & 12 & 77 \\
\hline Nylons & TapeLift & 9 & 13 & 72 \\
\hline Nylons & TapeLift & 9 & 14 & 42 \\
\hline Nylons & TapeLift & 9 & 15 & 48 \\
\hline Nylons & TapeLift & 9 & 16 & 20 \\
\hline Nylons & TapeLift & 9 & 17 & 40 \\
\hline Nylons & TapeLift & 9 & 18 & 110 \\
\hline Nylons & TapeLift & 9 & 19 & 41 \\
\hline Nylons & TapeLift & 9 & 20 & 18 \\
\hline Nylons & TapeLift & 10 & 1 & 24 \\
\hline Nylons & TapeLift & 10 & 2 & 29 \\
\hline Nylons & TapeLift & 10 & 3 & 25 \\
\hline Nylons & TapeLift & 10 & 4 & 81 \\
\hline Nylons & TapeLift & 10 & 5 & 12 \\
\hline Nylons & TapeLift & 10 & 6 & 146 \\
\hline Nylons & TapeLift & 10 & 7 & 50 \\
\hline Nylons & TapeLift & 10 & 8 & 33 \\
\hline Nylons & TapeLift & 10 & 9 & 90 \\
\hline Nylons & TapeLift & 10 & 10 & 78 \\
\hline Nylons & TapeLift & 10 & 11 & 59 \\
\hline Nylons & TapeLift & 10 & 12 & 64 \\
\hline Nylons & TapeLift & 10 & 13 & 25 \\
\hline Nylons & TapeLift & 10 & 14 & 98 \\
\hline Nylons & TapeLift & 10 & 15 & 68 \\
\hline Nylons & TapeLift & 10 & 16 & 83 \\
\hline Nylons & TapeLift & 10 & 17 & 91 \\
\hline Nylons & TapeLift & 10 & 18 & 71 \\
\hline Nylons & TapeLift & 10 & 19 & 96 \\
\hline Nylons & TapeLift & 10 & 20 & 46 \\
\hline Nylons & TapeLift & 10 & 21 & 54 \\
\hline
\end{tabular}




\begin{tabular}{|c|c|c|c|c|}
\hline Nylons & TapeLift & 10 & 22 & 151 \\
\hline Nylons & TapeLift & 10 & 23 & 89 \\
\hline Nylons & TapeLift & 10 & 24 & 77 \\
\hline Nylons & TapeLift & 10 & 25 & 82 \\
\hline Nylons & TapeLift & 11 & 1 & 8 \\
\hline Nylons & TapeLift & 11 & 2 & 9 \\
\hline Nylons & TapeLift & 11 & 3 & 19 \\
\hline Nylons & TapeLift & 11 & 4 & 24 \\
\hline Nylons & TapeLift & 11 & 5 & 32 \\
\hline Nylons & TapeLift & 11 & 6 & 33 \\
\hline Nylons & TapeLift & 11 & 7 & 57 \\
\hline Nylons & TapeLift & 11 & 8 & 37 \\
\hline Nylons & TapeLift & 11 & 9 & 53 \\
\hline Nylons & TapeLift & 11 & 10 & 117 \\
\hline Nylons & TapeLift & 11 & 11 & 83 \\
\hline Nylons & TapeLift & 11 & 12 & 29 \\
\hline Nylons & TapeLift & 11 & 13 & 73 \\
\hline Nylons & TapeLift & 11 & 14 & 46 \\
\hline Nylons & TapeLift & 11 & 15 & 69 \\
\hline Nylons & TapeLift & 11 & 16 & 25 \\
\hline Nylons & TapeLift & 11 & 17 & 131 \\
\hline Nylons & TapeLift & 11 & 18 & 140 \\
\hline Nylons & TapeLift & 11 & 19 & 86 \\
\hline Nylons & TapeLift & 11 & 20 & 70 \\
\hline Nylons & TapeLift & 11 & 21 & 66 \\
\hline Nylons & TapeLift & 11 & 22 & 64 \\
\hline Nylons & TapeLift & 11 & 23 & 97 \\
\hline Nylons & TapeLift & 11 & 24 & 71 \\
\hline Nylons & TapeLift & 11 & 25 & 78 \\
\hline Nylons & TapeLift & 12 & 1 & 47 \\
\hline Nylons & TapeLift & 12 & 2 & 38 \\
\hline Nylons & TapeLift & 12 & 3 & 23 \\
\hline Nylons & TapeLift & 12 & 4 & 25 \\
\hline Nylons & TapeLift & 12 & 5 & 21 \\
\hline Nylons & TapeLift & 12 & 6 & 60 \\
\hline Nylons & TapeLift & 12 & 7 & 14 \\
\hline Nylons & TapeLift & 12 & 8 & 19 \\
\hline Nylons & TapeLift & 12 & 9 & 70 \\
\hline Nylons & TapeLift & 12 & 10 & 84 \\
\hline Nylons & TapeLift & 12 & 11 & 72 \\
\hline Nylons & TapeLift & 12 & 12 & 30 \\
\hline Nylons & TapeLift & 12 & 13 & 23 \\
\hline Nylons & TapeLift & 12 & 14 & 41 \\
\hline Nylons & TapeLift & 12 & 15 & 44 \\
\hline Nylons & TapeLift & 12 & 16 & 65 \\
\hline
\end{tabular}




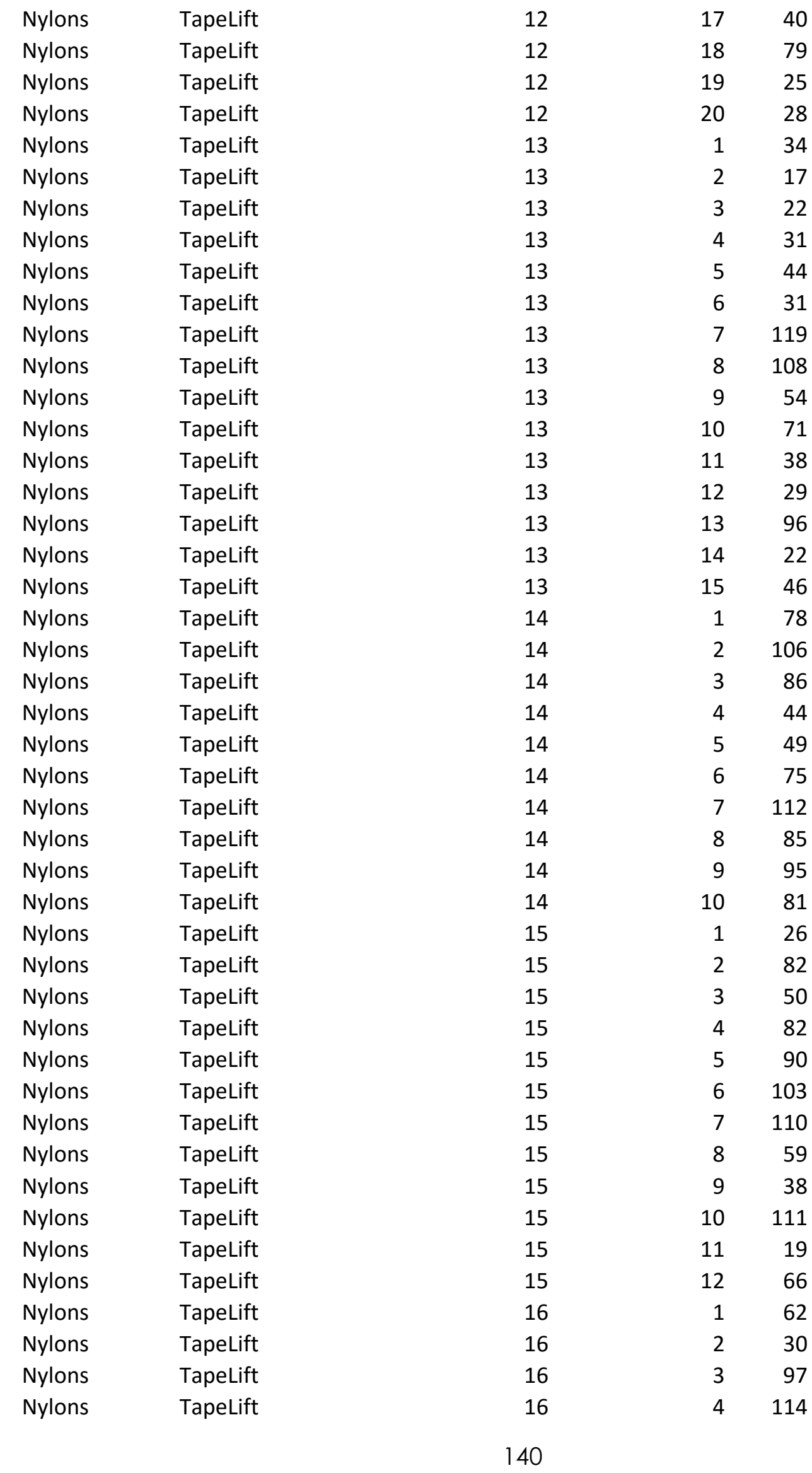




\begin{tabular}{|c|c|c|c|c|}
\hline Nylons & TapeLift & 16 & 5 & 31 \\
\hline Nylons & TapeLift & 16 & 6 & 48 \\
\hline Nylons & TapeLift & 16 & 7 & 74 \\
\hline Nylons & TapeLift & 16 & 8 & 136 \\
\hline Nylons & TapeLift & 16 & 9 & 104 \\
\hline Nylons & TapeLift & 16 & 10 & 52 \\
\hline Nylons & TapeLift & 16 & 11 & 55 \\
\hline Nylons & TapeLift & 16 & 12 & 122 \\
\hline Nylons & TapeLift & 16 & 13 & 60 \\
\hline Nylons & TapeLift & 16 & 14 & 20 \\
\hline Nylons & TapeLift & 16 & 15 & 55 \\
\hline Nylons & TapeLift & 17 & 1 & 35 \\
\hline Nylons & TapeLift & 17 & 2 & 32 \\
\hline Nylons & TapeLift & 17 & 3 & 137 \\
\hline Nylons & TapeLift & 17 & 4 & 39 \\
\hline Nylons & TapeLift & 17 & 5 & 58 \\
\hline Nylons & TapeLift & 17 & 6 & 94 \\
\hline Nylons & TapeLift & 17 & 7 & 18 \\
\hline Nylons & TapeLift & 17 & 8 & 95 \\
\hline Nylons & TapeLift & 17 & 9 & 101 \\
\hline Nylons & TapeLift & 17 & 10 & 59 \\
\hline Nylons & TapeLift & 17 & 11 & 150 \\
\hline Nylons & TapeLift & 17 & 12 & 66 \\
\hline Nylons & TapeLift & 17 & 13 & 37 \\
\hline Nylons & TapeLift & 17 & 14 & 74 \\
\hline Nylons & TapeLift & 17 & 15 & 18 \\
\hline Nylons & TapeLift & 18 & 1 & 76 \\
\hline Nylons & TapeLift & 18 & 2 & 38 \\
\hline Nylons & TapeLift & 18 & 3 & 80 \\
\hline Nylons & TapeLift & 18 & 4 & 92 \\
\hline Nylons & TapeLift & 18 & 5 & 29 \\
\hline Nylons & TapeLift & 18 & 6 & 23 \\
\hline Nylons & TapeLift & 18 & 7 & 91 \\
\hline Nylons & TapeLift & 18 & 8 & 28 \\
\hline Nylons & TapeLift & 18 & 9 & 27 \\
\hline Nylons & TapeLift & 18 & 10 & 13 \\
\hline Nylons & TapeLift & 18 & 11 & 34 \\
\hline Nylons & TapeLift & 18 & 12 & 110 \\
\hline Nylons & TapeLift & 18 & 13 & 38 \\
\hline Nylons & TapeLift & 18 & 14 & 17 \\
\hline Nylons & TapeLift & 18 & 15 & 122 \\
\hline Nylons & TapeLift & 19 & 1 & 23 \\
\hline Nylons & TapeLift & 19 & 2 & 15 \\
\hline Nylons & TapeLift & 19 & 3 & 14 \\
\hline Nylons & TapeLift & 19 & 4 & 33 \\
\hline
\end{tabular}




\begin{tabular}{|c|c|c|c|}
\hline Nylons & TapeLift & 19 & 5 \\
\hline Nylons & TapeLift & 19 & 6 \\
\hline Nylons & TapeLift & 19 & 7 \\
\hline Nylons & TapeLift & 19 & 8 \\
\hline Nylons & TapeLift & 19 & 9 \\
\hline Nylons & TapeLift & 19 & 10 \\
\hline Nylons & TapeLift & 19 & 11 \\
\hline Nylons & TapeLift & 19 & 12 \\
\hline Nylons & TapeLift & 19 & 13 \\
\hline Nylons & TapeLift & 19 & 14 \\
\hline Nylons & TapeLift & 19 & 15 \\
\hline Nylons & TapeLift & 19 & 16 \\
\hline Nylons & TapeLift & 19 & 17 \\
\hline Nylons & TapeLift & 19 & 18 \\
\hline Nylons & TapeLift & 19 & 19 \\
\hline Nylons & TapeLift & 19 & 20 \\
\hline Nylons & TapeLift & 20 & 1 \\
\hline Nylons & TapeLift & 20 & 2 \\
\hline Nylons & TapeLift & 20 & 3 \\
\hline Nylons & TapeLift & 20 & 4 \\
\hline Nylons & TapeLift & 20 & 5 \\
\hline Nylons & TapeLift & 20 & 6 \\
\hline Nylons & TapeLift & 20 & 7 \\
\hline Nylons & TapeLift & 20 & 8 \\
\hline Nylons & TapeLift & 20 & 9 \\
\hline Nylons & TapeLift & 20 & 10 \\
\hline Nylons & TapeLift & 20 & 11 \\
\hline Nylons & TapeLift & 20 & 12 \\
\hline Nylons & TapeLift & 20 & 13 \\
\hline Nylons & TapeLift & 20 & 14 \\
\hline Nylons & TapeLift & 20 & 15 \\
\hline Nylons & TapeLift & 20 & 16 \\
\hline Nylons & TapeLift & 20 & 17 \\
\hline Nylons & TapeLift & 20 & 18 \\
\hline Nylons & TapeLift & 20 & 19 \\
\hline Nylons & TapeLift & 20 & 20 \\
\hline Nylons & TapeLift & 20 & 21 \\
\hline Nylons & TapeLift & 20 & 22 \\
\hline Nylons & TapeLift & 20 & 23 \\
\hline Nylons & TapeLift & 20 & 24 \\
\hline Nylons & TapeLift & 20 & 25 \\
\hline Nylons & TapeLift & 20 & 26 \\
\hline Nylons & TapeLift & 20 & 27 \\
\hline Nylons & TapeLift & 20 & 28 \\
\hline Nylons & TapeLift & 20 & 29 \\
\hline
\end{tabular}




\begin{tabular}{|c|c|c|c|c|}
\hline Nylons & TapeLift & 20 & 30 & 62 \\
\hline Nylons & TapeLift & 21 & 1 & 31 \\
\hline Nylons & TapeLift & 21 & 2 & 67 \\
\hline Nylons & TapeLift & 21 & 3 & 31 \\
\hline Nylons & TapeLift & 21 & 4 & 50 \\
\hline Nylons & TapeLift & 21 & 5 & 57 \\
\hline Nylons & TapeLift & 21 & 6 & 99 \\
\hline Nylons & TapeLift & 21 & 7 & 15 \\
\hline Nylons & TapeLift & 21 & 8 & 58 \\
\hline Nylons & TapeLift & 21 & 9 & 20 \\
\hline Nylons & TapeLift & 21 & 10 & 27 \\
\hline Nylons & TapeLift & 21 & 11 & 31 \\
\hline Nylons & TapeLift & 21 & 12 & 32 \\
\hline Nylons & TapeLift & 21 & 13 & 30 \\
\hline Nylons & TapeLift & 21 & 14 & 31 \\
\hline Nylons & TapeLift & 21 & 15 & 183 \\
\hline Nylons & TapeLift & 21 & 16 & 49 \\
\hline Nylons & TapeLift & 21 & 17 & 133 \\
\hline Nylons & TapeLift & 21 & 18 & 117 \\
\hline Nylons & TapeLift & 21 & 19 & 52 \\
\hline Nylons & TapeLift & 21 & 20 & 102 \\
\hline Nylons & TapeLift & 22 & 1 & 42 \\
\hline Nylons & TapeLift & 22 & 2 & 64 \\
\hline Nylons & TapeLift & 22 & 3 & 56 \\
\hline Nylons & TapeLift & 22 & 4 & 101 \\
\hline Nylons & TapeLift & 22 & 5 & 49 \\
\hline Nylons & TapeLift & 22 & 6 & 48 \\
\hline Nylons & TapeLift & 22 & 7 & 72 \\
\hline Nylons & TapeLift & 22 & 8 & 79 \\
\hline Nylons & TapeLift & 22 & 9 & 112 \\
\hline Nylons & TapeLift & 22 & 10 & 69 \\
\hline Nylons & TapeLift & 22 & 11 & 94 \\
\hline Nylons & TapeLift & 22 & 12 & 66 \\
\hline Nylons & TapeLift & 22 & 13 & 54 \\
\hline Nylons & TapeLift & 22 & 14 & 37 \\
\hline Nylons & TapeLift & 22 & 15 & 52 \\
\hline Nylons & TapeLift & 23 & 1 & 62 \\
\hline Nylons & TapeLift & 23 & 2 & 66 \\
\hline Nylons & TapeLift & 23 & 3 & 46 \\
\hline Nylons & TapeLift & 23 & 4 & 131 \\
\hline Nylons & TapeLift & 23 & 5 & 74 \\
\hline Nylons & TapeLift & 23 & 6 & 26 \\
\hline Nylons & TapeLift & 23 & 7 & 97 \\
\hline Nylons & TapeLift & 23 & 8 & 83 \\
\hline Nylons & TapeLift & 23 & 9 & 63 \\
\hline
\end{tabular}




\begin{tabular}{|c|c|c|c|c|}
\hline Nylons & TapeLift & 23 & 10 & 85 \\
\hline Nylons & TapeLift & 24 & 1 & 94 \\
\hline Nylons & TapeLift & 24 & 2 & 71 \\
\hline Nylons & TapeLift & 24 & 3 & 57 \\
\hline Nylons & TapeLift & 24 & 4 & 81 \\
\hline Nylons & TapeLift & 24 & 5 & 81 \\
\hline Nylons & TapeLift & 24 & 6 & 79 \\
\hline Nylons & TapeLift & 24 & 7 & 75 \\
\hline Nylons & TapeLift & 24 & 8 & 53 \\
\hline Nylons & TapeLift & 24 & 9 & 73 \\
\hline Nylons & TapeLift & 24 & 10 & 123 \\
\hline Nylons & TapeLift & 24 & 11 & 30 \\
\hline Nylons & TapeLift & 24 & 12 & 47 \\
\hline Nylons & TapeLift & 24 & 13 & 58 \\
\hline Nylons & TapeLift & 24 & 14 & 43 \\
\hline Nylons & TapeLift & 24 & 15 & 81 \\
\hline Nylons & TapeLift & 24 & 16 & 49 \\
\hline Nylons & TapeLift & 24 & 17 & 41 \\
\hline Nylons & TapeLift & 24 & 18 & 59 \\
\hline Nylons & TapeLift & 25 & 1 & 68 \\
\hline Nylons & TapeLift & 25 & 2 & 73 \\
\hline Nylons & TapeLift & 25 & 3 & 71 \\
\hline Nylons & TapeLift & 25 & 4 & 92 \\
\hline Nylons & TapeLift & 25 & 5 & 69 \\
\hline Nylons & TapeLift & 25 & 6 & 123 \\
\hline Nylons & TapeLift & 25 & 7 & 51 \\
\hline Nylons & TapeLift & 25 & 8 & 27 \\
\hline Nylons & TapeLift & 25 & 9 & 27 \\
\hline Nylons & TapeLift & 25 & 10 & 36 \\
\hline Nylons & TapeLift & 25 & 11 & 74 \\
\hline Nylons & TapeLift & 25 & 12 & 87 \\
\hline Nylons & TapeLift & 25 & 13 & 46 \\
\hline Nylons & TapeLift & 25 & 14 & 60 \\
\hline Nylons & TapeLift & 25 & 15 & 8 \\
\hline Nylons & TapeLift & 25 & 16 & 42 \\
\hline Nylons & TapeLift & 25 & 17 & 24 \\
\hline Nylons & TapeLift & 25 & 18 & 80 \\
\hline Nylons & TapeLift & 26 & 1 & 47 \\
\hline Nylons & TapeLift & 26 & 2 & 95 \\
\hline Nylons & TapeLift & 26 & 3 & 58 \\
\hline Nylons & TapeLift & 26 & 4 & 28 \\
\hline Nylons & TapeLift & 26 & 5 & 70 \\
\hline Nylons & TapeLift & 26 & 6 & 176 \\
\hline Nylons & TapeLift & 26 & 7 & 121 \\
\hline Nylons & TapeLift & 26 & 8 & 105 \\
\hline
\end{tabular}




\begin{tabular}{|c|c|c|c|c|}
\hline Nylons & TapeLift & 26 & 9 & 115 \\
\hline Nylons & TapeLift & 26 & 10 & 80 \\
\hline Nylons & TapeLift & 26 & 11 & 83 \\
\hline Nylons & TapeLift & 26 & 12 & 35 \\
\hline Nylons & TapeLift & 26 & 13 & 108 \\
\hline Nylons & TapeLift & 26 & 14 & 94 \\
\hline Nylons & TapeLift & 26 & 15 & 92 \\
\hline Nylons & TapeLift & 26 & 16 & 18 \\
\hline Nylons & TapeLift & 26 & 17 & 56 \\
\hline Nylons & TapeLift & 26 & 18 & 69 \\
\hline Nylons & TapeLift & 26 & 19 & 115 \\
\hline Nylons & TapeLift & 26 & 20 & 107 \\
\hline Nylons & TapeLift & 27 & 1 & 80 \\
\hline Nylons & TapeLift & 27 & 2 & 78 \\
\hline Nylons & TapeLift & 27 & 3 & 84 \\
\hline Nylons & TapeLift & 27 & 4 & 41 \\
\hline Nylons & TapeLift & 27 & 5 & 82 \\
\hline Nylons & TapeLift & 27 & 6 & 108 \\
\hline Nylons & TapeLift & 27 & 7 & 61 \\
\hline Nylons & TapeLift & 27 & 8 & 54 \\
\hline Nylons & TapeLift & 27 & 9 & 76 \\
\hline Nylons & TapeLift & 27 & 10 & 78 \\
\hline Nylons & TapeLift & 27 & 11 & 69 \\
\hline Nylons & TapeLift & 27 & 12 & 84 \\
\hline Nylons & TapeLift & 27 & 13 & 95 \\
\hline Nylons & TapeLift & 27 & 14 & 51 \\
\hline Nylons & TapeLift & 27 & 15 & 88 \\
\hline Nylons & TapeLift & 27 & 16 & 78 \\
\hline Nylons & TapeLift & 27 & 17 & 82 \\
\hline Nylons & TapeLift & 27 & 18 & 93 \\
\hline Nylons & TapeLift & 27 & 19 & 111 \\
\hline Nylons & TapeLift & 27 & 20 & 52 \\
\hline Nylons & TapeLift & 27 & 21 & 68 \\
\hline Nylons & TapeLift & 27 & 22 & 105 \\
\hline Nylons & TapeLift & 27 & 23 & 72 \\
\hline Nylons & TapeLift & 27 & 24 & 110 \\
\hline Nylons & TapeLift & 28 & 1 & 67 \\
\hline Nylons & TapeLift & 28 & 2 & 44 \\
\hline Nylons & TapeLift & 28 & 3 & 88 \\
\hline Nylons & TapeLift & 28 & 4 & 73 \\
\hline Nylons & TapeLift & 28 & 5 & 77 \\
\hline Nylons & TapeLift & 28 & 6 & 84 \\
\hline Nylons & TapeLift & 28 & 7 & 119 \\
\hline Nylons & TapeLift & 28 & 8 & 79 \\
\hline Nylons & TapeLift & 28 & 9 & 24 \\
\hline
\end{tabular}




\begin{tabular}{|c|c|c|c|c|}
\hline Nylons & TapeLift & 28 & 10 & 62 \\
\hline Nylons & TapeLift & 28 & 11 & 97 \\
\hline Nylons & TapeLift & 28 & 12 & 65 \\
\hline Nylons & TapeLift & 28 & 13 & 78 \\
\hline Nylons & TapeLift & 28 & 14 & 64 \\
\hline Nylons & TapeLift & 28 & 15 & 84 \\
\hline Nylons & TapeLift & 28 & 16 & 57 \\
\hline Nylons & TapeLift & 28 & 17 & 117 \\
\hline Nylons & TapeLift & 28 & 18 & 53 \\
\hline Nylons & TapeLift & 29 & 1 & 45 \\
\hline Nylons & TapeLift & 29 & 2 & 88 \\
\hline Nylons & TapeLift & 29 & 3 & 170 \\
\hline Nylons & TapeLift & 29 & 4 & 99 \\
\hline Nylons & TapeLift & 29 & 5 & 26 \\
\hline Nylons & TapeLift & 29 & 6 & 47 \\
\hline Nylons & TapeLift & 29 & 7 & 100 \\
\hline Nylons & TapeLift & 29 & 8 & 63 \\
\hline Nylons & TapeLift & 29 & 9 & 104 \\
\hline Nylons & TapeLift & 29 & 10 & 103 \\
\hline Nylons & TapeLift & 29 & 11 & 50 \\
\hline Nylons & TapeLift & 29 & 12 & 80 \\
\hline Nylons & TapeLift & 29 & 13 & 83 \\
\hline Nylons & TapeLift & 29 & 14 & 47 \\
\hline Nylons & TapeLift & 29 & 15 & 58 \\
\hline Nylons & TapeLift & 29 & 16 & 121 \\
\hline Nylons & TapeLift & 29 & 17 & 73 \\
\hline Nylons & TapeLift & 29 & 18 & 41 \\
\hline Nylons & TapeLift & 29 & 19 & 29 \\
\hline Nylons & TapeLift & 29 & 20 & 84 \\
\hline Nylons & TapeLift & 30 & 1 & 67 \\
\hline Nylons & TapeLift & 30 & 2 & 63 \\
\hline Nylons & TapeLift & 30 & 3 & 69 \\
\hline Nylons & TapeLift & 30 & 4 & 53 \\
\hline Nylons & TapeLift & 30 & 5 & 72 \\
\hline Nylons & TapeLift & 30 & 6 & 66 \\
\hline Nylons & TapeLift & 30 & 7 & 45 \\
\hline Nylons & TapeLift & 30 & 8 & 63 \\
\hline Nylons & TapeLift & 30 & 9 & 86 \\
\hline Nylons & TapeLift & 30 & 10 & 50 \\
\hline Nylons & TapeLift & 30 & 11 & 38 \\
\hline Nylons & TapeLift & 30 & 12 & 59 \\
\hline Nylons & TapeLift & 30 & 13 & 74 \\
\hline Nylons & TapeLift & 30 & 14 & 66 \\
\hline Nylons & TapeLift & 30 & 15 & 36 \\
\hline Nylons & TapeLift & 30 & 16 & 56 \\
\hline
\end{tabular}




\begin{tabular}{|c|c|c|c|}
\hline Nylons & TapeLift & 30 & 17 \\
\hline Nylons & TapeLift & 30 & 18 \\
\hline Nylons & TapeLift & 30 & 19 \\
\hline Nylons & TapeLift & 30 & 20 \\
\hline Nylons & TapeLift & 30 & 21 \\
\hline Nylons & TapeLift & 30 & 22 \\
\hline Nylons & TapeLift & 30 & 23 \\
\hline Nylons & TapeLift & 30 & 24 \\
\hline Nylons & TapeLift & 30 & 25 \\
\hline Nylons & TapeLift & 31 & 1 \\
\hline Nylons & TapeLift & 31 & 2 \\
\hline Nylons & TapeLift & 31 & 3 \\
\hline Nylons & TapeLift & 31 & 4 \\
\hline Nylons & TapeLift & 31 & 5 \\
\hline Nylons & TapeLift & 31 & 6 \\
\hline Nylons & TapeLift & 31 & 7 \\
\hline Nylons & TapeLift & 31 & 8 \\
\hline Nylons & TapeLift & 31 & 9 \\
\hline Nylons & TapeLift & 31 & 10 \\
\hline Nylons & TapeLift & 31 & 11 \\
\hline Nylons & TapeLift & 31 & 12 \\
\hline Nylons & TapeLift & 31 & 13 \\
\hline Nylons & TapeLift & 31 & 14 \\
\hline Nylons & TapeLift & 31 & 15 \\
\hline Nylons & TapeLift & 31 & 16 \\
\hline Nylons & TapeLift & 31 & 17 \\
\hline Nylons & TapeLift & 31 & 18 \\
\hline Nylons & TapeLift & 31 & 19 \\
\hline Nylons & TapeLift & 31 & 20 \\
\hline Nylons & TapeLift & 31 & 21 \\
\hline Nylons & TapeLift & 31 & 22 \\
\hline Nylons & TapeLift & 31 & 23 \\
\hline Nylons & TapeLift & 31 & 24 \\
\hline Nylons & TapeLift & 31 & 25 \\
\hline Nylons & TapeLift & 31 & 26 \\
\hline Nylons & TapeLift & 31 & 27 \\
\hline Nylons & TapeLift & 31 & 28 \\
\hline Nylons & TapeLift & 31 & 29 \\
\hline Nylons & TapeLift & 31 & 30 \\
\hline Nylons & TapeLift & 31 & 31 \\
\hline Nylons & TapeLift & 31 & 32 \\
\hline Nylons & TapeLift & 31 & 33 \\
\hline Nylons & TapeLift & 31 & 34 \\
\hline Nylons & TapeLift & 31 & 35 \\
\hline Nylons & TapeLift & 31 & 36 \\
\hline
\end{tabular}




\begin{tabular}{|c|c|c|c|}
\hline Nylons & TapeLift & 31 & 37 \\
\hline Nylons & TapeLift & 31 & 38 \\
\hline Nylons & TapeLift & 31 & 39 \\
\hline Nylons & TapeLift & 31 & 40 \\
\hline Nylons & TapeLift & 31 & 41 \\
\hline Nylons & TapeLift & 31 & 42 \\
\hline Nylons & TapeLift & 31 & 43 \\
\hline Nylons & TapeLift & 31 & 44 \\
\hline Nylons & TapeLift & 32 & 1 \\
\hline Nylons & TapeLift & 32 & 2 \\
\hline Nylons & TapeLift & 32 & 3 \\
\hline Nylons & TapeLift & 32 & 4 \\
\hline Nylons & TapeLift & 32 & 5 \\
\hline Nylons & TapeLift & 32 & 6 \\
\hline Nylons & TapeLift & 32 & 7 \\
\hline Nylons & TapeLift & 32 & 8 \\
\hline Nylons & TapeLift & 33 & 1 \\
\hline Nylons & TapeLift & 33 & 2 \\
\hline Nylons & TapeLift & 33 & 3 \\
\hline Nylons & TapeLift & 33 & 4 \\
\hline Nylons & TapeLift & 33 & 5 \\
\hline Nylons & TapeLift & 33 & 6 \\
\hline Nylons & TapeLift & 33 & 7 \\
\hline Nylons & TapeLift & 33 & 8 \\
\hline Nylons & TapeLift & 33 & 9 \\
\hline Nylons & TapeLift & 33 & 10 \\
\hline Nylons & TapeLift & 33 & 11 \\
\hline Nylons & TapeLift & 33 & 12 \\
\hline Nylons & TapeLift & 33 & 13 \\
\hline Nylons & TapeLift & 33 & 14 \\
\hline Nylons & TapeLift & 33 & 15 \\
\hline Nylons & TapeLift & 33 & 16 \\
\hline Nylons & TapeLift & 33 & 17 \\
\hline Nylons & TapeLift & 34 & 1 \\
\hline Nylons & TapeLift & 34 & 2 \\
\hline Nylons & TapeLift & 34 & 3 \\
\hline Nylons & TapeLift & 34 & 4 \\
\hline Nylons & TapeLift & 34 & 5 \\
\hline Nylons & TapeLift & 34 & 6 \\
\hline Nylons & TapeLift & 34 & 7 \\
\hline Nylons & TapeLift & 34 & 8 \\
\hline Nylons & TapeLift & 34 & 9 \\
\hline Nylons & TapeLift & 34 & 10 \\
\hline Nylons & TapeLift & 34 & 11 \\
\hline Nylons & TapeLift & 34 & 12 \\
\hline
\end{tabular}




\begin{tabular}{|c|c|c|c|}
\hline Nylons & TapeLift & 35 & 1 \\
\hline Nylons & TapeLift & 35 & 2 \\
\hline Nylons & TapeLift & 35 & 3 \\
\hline Nylons & TapeLift & 35 & 4 \\
\hline Nylons & TapeLift & 35 & 5 \\
\hline Nylons & TapeLift & 35 & 6 \\
\hline Nylons & TapeLift & 35 & 7 \\
\hline Nylons & TapeLift & 35 & 8 \\
\hline Nylons & TapeLift & 35 & 9 \\
\hline Nylons & TapeLift & 35 & 10 \\
\hline Nylons & TapeLift & 35 & 11 \\
\hline Nylons & TapeLift & 35 & 12 \\
\hline Nylons & TapeLift & 35 & 13 \\
\hline Nylons & TapeLift & 35 & 14 \\
\hline Nylons & TapeLift & 35 & 15 \\
\hline Nylons & TapeLift & 35 & 16 \\
\hline Nylons & TapeLift & 35 & 17 \\
\hline Nylons & TapeLift & 35 & 18 \\
\hline Nylons & TapeLift & 35 & 19 \\
\hline Nylons & TapeLift & 35 & 20 \\
\hline Nylons & TapeLift & 35 & 21 \\
\hline Nylons & TapeLift & 35 & 22 \\
\hline Nylons & TapeLift & 35 & 23 \\
\hline Nylons & TapeLift & 35 & 24 \\
\hline Nylons & TapeLift & 35 & 25 \\
\hline Nylons & Vacuum & 1 & 1 \\
\hline Nylons & Vacuum & 1 & 2 \\
\hline Nylons & Vacuum & 1 & 3 \\
\hline Nylons & Vacuum & 1 & 4 \\
\hline Nylons & Vacuum & 1 & 5 \\
\hline Nylons & Vacuum & 1 & 6 \\
\hline Nylons & Vacuum & 1 & 7 \\
\hline Nylons & Vacuum & 1 & 8 \\
\hline Nylons & Vacuum & 1 & 9 \\
\hline Nylons & Vacuum & 1 & 10 \\
\hline Nylons & Vacuum & 1 & 11 \\
\hline Nylons & Vacuum & 1 & 12 \\
\hline Nylons & Vacuum & 1 & 13 \\
\hline Nylons & Vacuum & 1 & 14 \\
\hline Nylons & Vacuum & 1 & 15 \\
\hline Nylons & Vacuum & 1 & 16 \\
\hline Nylons & Vacuum & 1 & 17 \\
\hline Nylons & Vacuum & 1 & 18 \\
\hline Nylons & Vacuum & 1 & 19 \\
\hline Nylons & Vacuum & 1 & 20 \\
\hline
\end{tabular}




\begin{tabular}{|c|c|}
\hline Nylons & Vacuum \\
\hline Nylons & Vacul \\
\hline Nylons & Vacu \\
\hline Nylons & Vacu \\
\hline Nylons & Vacuu \\
\hline Nylons & vacue \\
\hline Nylons & Vacul \\
\hline Nylons & Vacul \\
\hline Nylons & Vacu \\
\hline Nylons & Vacuu \\
\hline Nylons & Vacuu \\
\hline Nylons & Vacus \\
\hline Nylons & Vacul \\
\hline Nylons & Vacu \\
\hline Nylons & Vacu \\
\hline Nylons & Vacu \\
\hline Nylons & Vacu \\
\hline Nylons & Vacul \\
\hline Nylons & Vacus \\
\hline Nylons & Vacu \\
\hline Nylons & Vacu \\
\hline Nylons & Vacu \\
\hline Nylons & Vacuu \\
\hline Nylons & Vacuu \\
\hline Nylons & Vacu \\
\hline Nylons & Vacuu \\
\hline Nylons & Vacuu \\
\hline Nylons & Vacuu \\
\hline Nylons & Vacuu \\
\hline Nylons & Vacul \\
\hline Nylons & Vacu \\
\hline Nylons & Vacu \\
\hline Nylons & Vacuu \\
\hline Nylons & Vacul \\
\hline Nylons & Vacuu \\
\hline Nylons & Vacu \\
\hline Nylons & Vacu \\
\hline Nylons & Vacuu \\
\hline Nylons & Vacuu \\
\hline Nylons & Vacuur \\
\hline Nylons & Vacuu \\
\hline Nylons & Vacuu \\
\hline Nylons & Vacul \\
\hline Nylons & Vacuu \\
\hline Nylons & Vacu \\
\hline
\end{tabular}

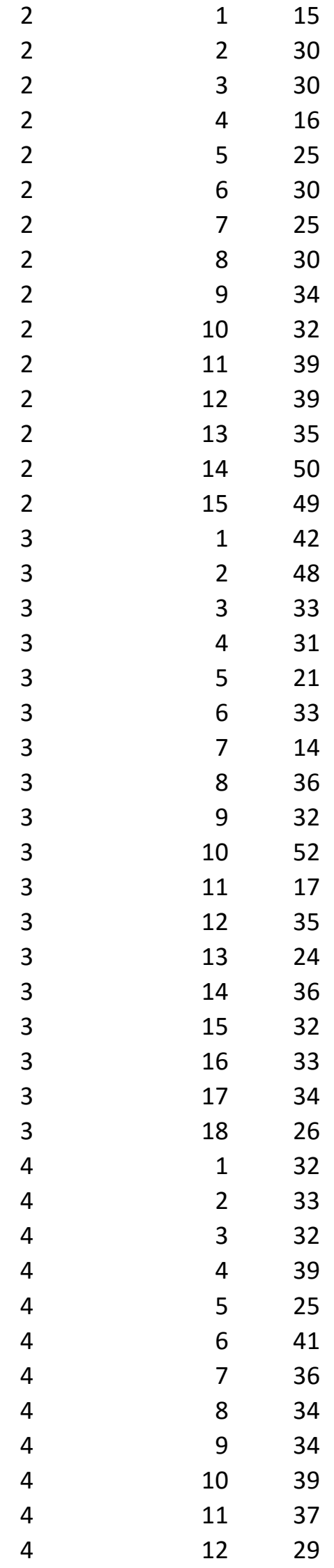




\begin{tabular}{|c|c|c|c|c|}
\hline Nylons & Vacuum & 4 & 13 & 25 \\
\hline Nylons & Vacuum & 4 & 14 & 38 \\
\hline Nylons & Vacuum & 4 & 15 & 34 \\
\hline Nylons & Vacuum & 5 & 1 & 41 \\
\hline Nylons & Vacuum & 5 & 2 & 19 \\
\hline Nylons & Vacuum & 5 & 3 & 35 \\
\hline Nylons & Vacuum & 5 & 4 & 57 \\
\hline Nylons & Vacuum & 5 & 5 & 40 \\
\hline Nylons & Vacuum & 5 & 6 & 55 \\
\hline Nylons & Vacuum & 5 & 7 & 37 \\
\hline Nylons & Vacuum & 5 & 8 & 38 \\
\hline Nylons & Vacuum & 5 & 9 & 34 \\
\hline Nylons & Vacuum & 5 & 10 & 30 \\
\hline Nylons & Vacuum & 5 & 11 & 29 \\
\hline Nylons & Vacuum & 5 & 12 & 35 \\
\hline Nylons & Vacuum & 6 & 1 & 23 \\
\hline Nylons & Vacuum & 6 & 2 & 24 \\
\hline Nylons & Vacuum & 6 & 3 & 25 \\
\hline Nylons & Vacuum & 6 & 4 & 17 \\
\hline Nylons & Vacuum & 6 & 5 & 19 \\
\hline Nylons & Vacuum & 6 & 6 & 5 \\
\hline Nylons & Vacuum & 6 & 7 & 17 \\
\hline Nylons & Vacuum & 6 & 8 & 38 \\
\hline Nylons & Vacuum & 6 & 9 & 15 \\
\hline Nylons & Vacuum & 6 & 10 & 36 \\
\hline Nylons & Vacuum & 7 & 1 & 21 \\
\hline Nylons & Vacuum & 7 & 2 & 39 \\
\hline Nylons & Vacuum & 7 & 3 & 19 \\
\hline Nylons & Vacuum & 7 & 4 & 28 \\
\hline Nylons & Vacuum & 7 & 5 & 24 \\
\hline Nylons & Vacuum & 7 & 6 & 42 \\
\hline Nylons & Vacuum & 7 & 7 & 34 \\
\hline Nylons & Vacuum & 7 & 8 & 36 \\
\hline Nylons & Vacuum & 7 & 9 & 42 \\
\hline Nylons & Vacuum & 7 & 10 & 33 \\
\hline Nylons & Vacuum & 7 & 11 & 13 \\
\hline Nylons & Vacuum & 7 & 12 & 30 \\
\hline Nylons & Vacuum & 7 & 13 & 26 \\
\hline Nylons & Vacuum & 7 & 14 & 26 \\
\hline Nylons & Vacuum & 7 & 15 & 41 \\
\hline Nylons & Vacuum & 8 & 1 & 61 \\
\hline Nylons & Vacuum & 8 & 2 & 51 \\
\hline Nylons & Vacuum & 8 & 3 & 50 \\
\hline Nylons & Vacuum & 8 & 4 & 34 \\
\hline Nylons & Vacuum & 8 & 5 & 45 \\
\hline
\end{tabular}




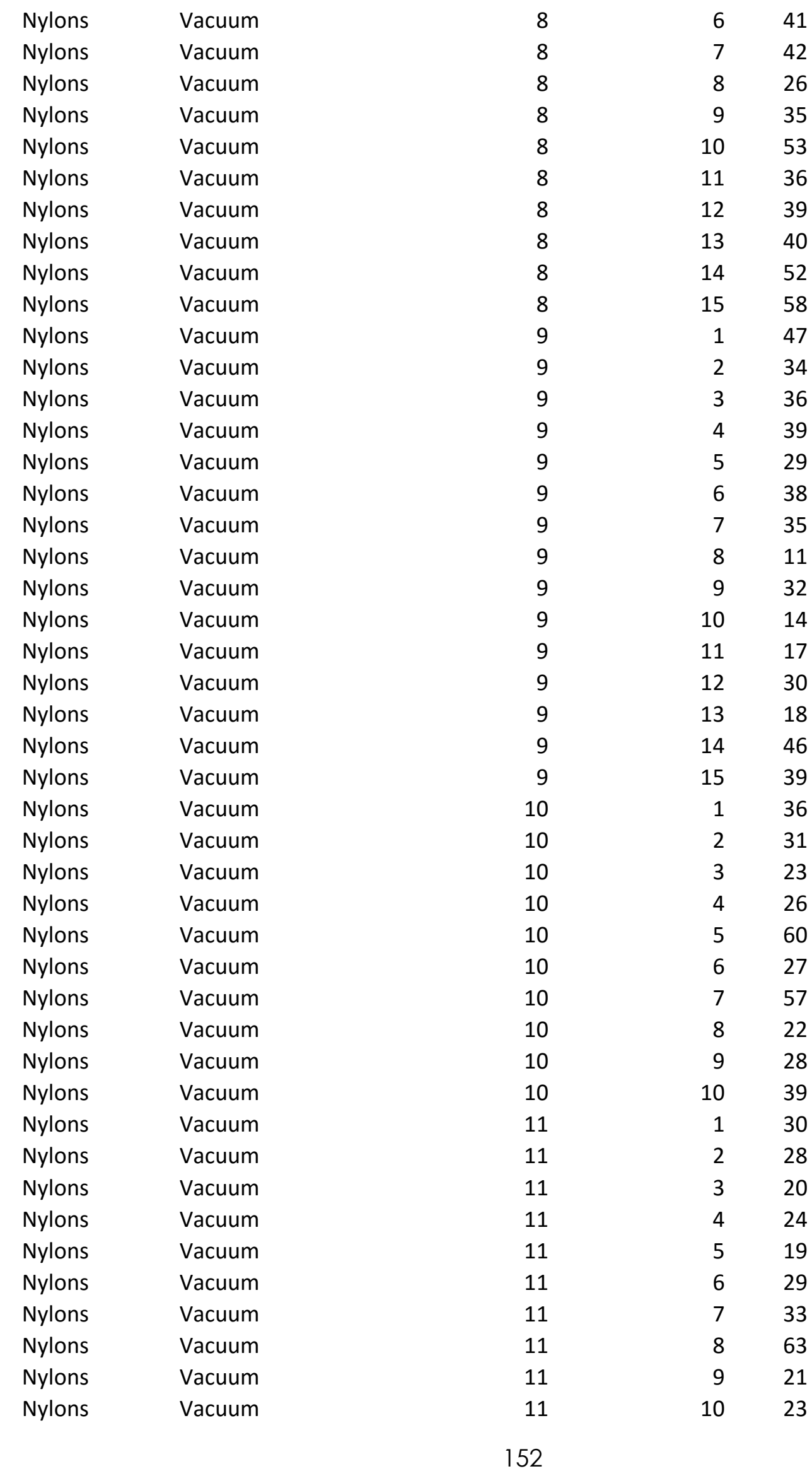




\begin{tabular}{|c|c|c|c|c|}
\hline Nylons & Vacuum & 11 & 11 & 39 \\
\hline Nylons & Vacuum & 12 & 1 & 22 \\
\hline Nylons & Vacuum & 12 & 2 & 23 \\
\hline Nylons & Vacuum & 12 & 3 & 36 \\
\hline Nylons & Vacuum & 12 & 4 & 35 \\
\hline Nylons & Vacuum & 12 & 5 & 38 \\
\hline Nylons & Vacuum & 12 & 6 & 23 \\
\hline Nylons & Vacuum & 12 & 7 & 23 \\
\hline Nylons & Vacuum & 12 & 8 & 41 \\
\hline Nylons & Vacuum & 12 & 9 & 12 \\
\hline Nylons & Vacuum & 12 & 10 & 31 \\
\hline Nylons & Vacuum & 12 & 11 & 11 \\
\hline Nylons & Vacuum & 12 & 12 & 23 \\
\hline Nylons & Vacuum & 12 & 13 & 32 \\
\hline Nylons & Vacuum & 13 & 1 & 22 \\
\hline Nylons & Vacuum & 13 & 2 & 32 \\
\hline Nylons & Vacuum & 13 & 3 & 27 \\
\hline Nylons & Vacuum & 13 & 4 & 32 \\
\hline Nylons & Vacuum & 13 & 5 & 12 \\
\hline Nylons & Vacuum & 13 & 6 & 30 \\
\hline Nylons & Vacuum & 13 & 7 & 29 \\
\hline Nylons & Vacuum & 13 & 8 & 28 \\
\hline Nylons & Vacuum & 13 & 9 & 9 \\
\hline Nylons & Vacuum & 13 & 10 & 19 \\
\hline Nylons & Vacuum & 13 & 11 & 31 \\
\hline Nylons & Vacuum & 13 & 12 & 32 \\
\hline Nylons & Vacuum & 13 & 13 & 41 \\
\hline Nylons & Vacuum & 13 & 14 & 25 \\
\hline Nylons & Vacuum & 13 & 15 & 34 \\
\hline Nylons & Vacuum & 13 & 16 & 51 \\
\hline Nylons & Vacuum & 14 & 1 & 49 \\
\hline Nylons & Vacuum & 14 & 2 & 40 \\
\hline Nylons & Vacuum & 14 & 3 & 39 \\
\hline Nylons & Vacuum & 14 & 4 & 39 \\
\hline Nylons & Vacuum & 14 & 5 & 22 \\
\hline Nylons & Vacuum & 14 & 6 & 36 \\
\hline Nylons & Vacuum & 14 & 7 & 53 \\
\hline Nylons & Vacuum & 14 & 8 & 41 \\
\hline Nylons & Vacuum & 14 & 9 & 22 \\
\hline Nylons & Vacuum & 14 & 10 & 59 \\
\hline Nylons & Vacuum & 15 & 1 & 43 \\
\hline Nylons & Vacuum & 15 & 2 & 25 \\
\hline Nylons & Vacuum & 15 & 3 & 19 \\
\hline Nylons & Vacuum & 15 & 4 & 32 \\
\hline Nylons & Vacuum & 15 & 5 & 12 \\
\hline
\end{tabular}




\begin{tabular}{|c|c|c|c|c|}
\hline Nylons & Vacuum & 15 & 6 & 31 \\
\hline Nylons & Vacuum & 15 & 7 & 48 \\
\hline Nylons & Vacuum & 15 & 8 & 12 \\
\hline Nylons & Vacuum & 15 & 9 & 37 \\
\hline Nylons & Vacuum & 16 & 1 & 37 \\
\hline Nylons & Vacuum & 16 & 2 & 37 \\
\hline Nylons & Vacuum & 16 & 3 & 37 \\
\hline Nylons & Vacuum & 16 & 4 & 12 \\
\hline Nylons & Vacuum & 16 & 5 & 24 \\
\hline Nylons & Vacuum & 16 & 6 & 19 \\
\hline Nylons & Vacuum & 16 & 7 & 47 \\
\hline Nylons & Vacuum & 16 & 8 & 30 \\
\hline Nylons & Vacuum & 16 & 9 & 20 \\
\hline Nylons & Vacuum & 16 & 10 & 34 \\
\hline Nylons & Vacuum & 16 & 11 & 73 \\
\hline Nylons & Vacuum & 16 & 12 & 30 \\
\hline Nylons & Vacuum & 16 & 13 & 16 \\
\hline Nylons & Vacuum & 16 & 14 & 22 \\
\hline Nylons & Vacuum & 16 & 15 & 13 \\
\hline Nylons & Vacuum & 16 & 16 & 22 \\
\hline Nylons & Vacuum & 17 & 1 & 21 \\
\hline Nylons & Vacuum & 17 & 2 & 30 \\
\hline Nylons & Vacuum & 17 & 3 & 23 \\
\hline Nylons & Vacuum & 17 & 4 & 17 \\
\hline Nylons & Vacuum & 17 & 5 & 26 \\
\hline Nylons & Vacuum & 17 & 6 & 36 \\
\hline Nylons & Vacuum & 17 & 7 & 23 \\
\hline Nylons & Vacuum & 17 & 8 & 16 \\
\hline Nylons & Vacuum & 17 & 9 & 25 \\
\hline Nylons & Vacuum & 17 & 10 & 15 \\
\hline Nylons & Vacuum & 17 & 11 & 19 \\
\hline Nylons & Vacuum & 17 & 12 & 31 \\
\hline Nylons & Vacuum & 17 & 13 & 55 \\
\hline Nylons & Vacuum & 17 & 14 & 39 \\
\hline Nylons & Vacuum & 17 & 15 & 39 \\
\hline Nylons & Vacuum & 17 & 16 & 13 \\
\hline Nylons & Vacuum & 17 & 17 & 19 \\
\hline Nylons & Vacuum & 18 & 1 & 33 \\
\hline Nylons & Vacuum & 18 & 2 & 37 \\
\hline Nylons & Vacuum & 18 & 3 & 25 \\
\hline Nylons & Vacuum & 18 & 4 & 25 \\
\hline Nylons & Vacuum & 18 & 5 & 22 \\
\hline Nylons & Vacuum & 18 & 6 & 12 \\
\hline Nylons & Vacuum & 18 & 7 & 34 \\
\hline Nylons & Vacuum & 18 & 8 & 10 \\
\hline
\end{tabular}




\begin{tabular}{|c|c|c|c|c|}
\hline Nylons & Vacuum & 18 & 9 & 9 \\
\hline Nylons & Vacuum & 18 & 10 & 32 \\
\hline Nylons & Vacuum & 18 & 11 & 35 \\
\hline Nylons & Vacuum & 18 & 12 & 34 \\
\hline Nylons & Vacuum & 18 & 13 & 73 \\
\hline Nylons & Vacuum & 18 & 14 & 52 \\
\hline Nylons & Vacuum & 18 & 15 & 31 \\
\hline Nylons & Vacuum & 19 & 1 & 35 \\
\hline Nylons & Vacuum & 19 & 2 & 36 \\
\hline Nylons & Vacuum & 19 & 3 & 15 \\
\hline Nylons & Vacuum & 19 & 4 & 64 \\
\hline Nylons & Vacuum & 19 & 5 & 28 \\
\hline Nylons & Vacuum & 19 & 6 & 72 \\
\hline Nylons & Vacuum & 19 & 7 & 38 \\
\hline Nylons & Vacuum & 19 & 8 & 46 \\
\hline Nylons & Vacuum & 19 & 9 & 11 \\
\hline Nylons & Vacuum & 19 & 10 & 57 \\
\hline Nylons & Vacuum & 19 & 11 & 23 \\
\hline Nylons & Vacuum & 19 & 12 & 14 \\
\hline Nylons & Vacuum & 19 & 13 & 81 \\
\hline Nylons & Vacuum & 19 & 14 & 48 \\
\hline Nylons & Vacuum & 19 & 15 & 34 \\
\hline Nylons & Vacuum & 19 & 16 & 28 \\
\hline Nylons & Vacuum & 20 & 1 & 27 \\
\hline Nylons & Vacuum & 20 & 2 & 30 \\
\hline Nylons & Vacuum & 20 & 3 & 43 \\
\hline Nylons & Vacuum & 20 & 4 & 32 \\
\hline Nylons & Vacuum & 20 & 5 & 26 \\
\hline Nylons & Vacuum & 20 & 6 & 12 \\
\hline Nylons & Vacuum & 20 & 7 & 9 \\
\hline Nylons & Vacuum & 20 & 8 & 37 \\
\hline Nylons & Vacuum & 20 & 9 & 28 \\
\hline Nylons & Vacuum & 20 & 10 & 23 \\
\hline Nylons & Vacuum & 20 & 11 & 43 \\
\hline Nylons & Vacuum & 20 & 12 & 13 \\
\hline Nylons & Vacuum & 20 & 13 & 20 \\
\hline Nylons & Vacuum & 20 & 14 & 48 \\
\hline Nylons & Vacuum & 21 & 1 & 39 \\
\hline Nylons & Vacuum & 21 & 2 & 22 \\
\hline Nylons & Vacuum & 21 & 3 & 31 \\
\hline Nylons & Vacuum & 21 & 4 & 10 \\
\hline Nylons & Vacuum & 21 & 5 & 40 \\
\hline Nylons & Vacuum & 21 & 6 & 44 \\
\hline Nylons & Vacuum & 21 & 7 & 43 \\
\hline Nylons & Vacuum & 21 & 8 & 26 \\
\hline
\end{tabular}




\begin{tabular}{|c|c|c|c|}
\hline Nylons & Vacuum & 21 & 9 \\
\hline Nylons & Vacuum & 21 & 10 \\
\hline Nylons & Vacuum & 21 & 11 \\
\hline Nylons & Vacuum & 21 & 12 \\
\hline Nylons & Vacuum & 21 & 13 \\
\hline Nylons & Vacuum & 21 & 14 \\
\hline Nylons & Vacuum & 21 & 15 \\
\hline Nylons & Vacuum & 21 & 16 \\
\hline Nylons & Vacuum & 21 & 17 \\
\hline Nylons & Vacuum & 21 & 18 \\
\hline Nylons & Vacuum & 22 & 1 \\
\hline Nylons & Vacuum & 22 & 2 \\
\hline Nylons & Vacuum & 22 & 3 \\
\hline Nylons & Vacuum & 22 & 4 \\
\hline Nylons & Vacuum & 22 & 5 \\
\hline Nylons & Vacuum & 22 & 6 \\
\hline Nylons & Vacuum & 22 & 7 \\
\hline Nylons & Vacuum & 22 & 8 \\
\hline Nylons & Vacuum & 22 & 9 \\
\hline Nylons & Vacuum & 22 & 10 \\
\hline Nylons & Vacuum & 22 & 11 \\
\hline Nylons & Vacuum & 22 & 12 \\
\hline Nylons & Vacuum & 22 & 13 \\
\hline Nylons & Vacuum & 22 & 14 \\
\hline Nylons & Vacuum & 23 & 1 \\
\hline Nylons & Vacuum & 23 & 2 \\
\hline Nylons & Vacuum & 23 & 3 \\
\hline Nylons & Vacuum & 23 & 4 \\
\hline Nylons & Vacuum & 23 & 5 \\
\hline Nylons & Vacuum & 23 & 6 \\
\hline Nylons & Vacuum & 23 & 7 \\
\hline Nylons & Vacuum & 23 & 8 \\
\hline Nylons & Vacuum & 24 & 1 \\
\hline Nylons & Vacuum & 24 & 2 \\
\hline Nylons & Vacuum & 24 & 3 \\
\hline Nylons & Vacuum & 24 & 4 \\
\hline Nylons & Vacuum & 24 & 5 \\
\hline Nylons & Vacuum & 24 & 6 \\
\hline Nylons & Vacuum & 24 & 7 \\
\hline Nylons & Vacuum & 24 & 8 \\
\hline Nylons & Vacuum & 24 & 9 \\
\hline Nylons & Vacuum & 24 & 10 \\
\hline Nylons & Vacuum & 25 & 1 \\
\hline Nylons & Vacuum & 25 & 2 \\
\hline Nylons & Vacuum & 25 & 3 \\
\hline
\end{tabular}




\begin{tabular}{|c|c|c|c|c|}
\hline Nylons & Vacuum & 25 & 4 & 36 \\
\hline Nylons & Vacuum & 25 & 5 & 26 \\
\hline Nylons & Vacuum & 25 & 6 & 35 \\
\hline Nylons & Vacuum & 25 & 7 & 37 \\
\hline Nylons & Vacuum & 25 & 8 & 26 \\
\hline Nylons & Vacuum & 25 & 9 & 37 \\
\hline Nylons & Vacuum & 25 & 10 & 24 \\
\hline Nylons & Vacuum & 25 & 11 & 42 \\
\hline Nylons & Vacuum & 25 & 12 & 59 \\
\hline Nylons & Vacuum & 25 & 13 & 46 \\
\hline Nylons & Vacuum & 25 & 14 & 37 \\
\hline Nylons & Vacuum & 26 & 1 & 40 \\
\hline Nylons & Vacuum & 26 & 2 & 33 \\
\hline Nylons & Vacuum & 26 & 3 & 29 \\
\hline Nylons & Vacuum & 26 & 4 & 37 \\
\hline Nylons & Vacuum & 26 & 5 & 39 \\
\hline Nylons & Vacuum & 26 & 6 & 52 \\
\hline Nylons & Vacuum & 26 & 7 & 32 \\
\hline Nylons & Vacuum & 26 & 8 & 25 \\
\hline Nylons & Vacuum & 26 & 9 & 54 \\
\hline Nylons & Vacuum & 26 & 10 & 38 \\
\hline Nylons & Vacuum & 26 & 11 & 35 \\
\hline Nylons & Vacuum & 26 & 12 & 33 \\
\hline Nylons & Vacuum & 26 & 13 & 41 \\
\hline Nylons & Vacuum & 27 & 1 & 27 \\
\hline Nylons & Vacuum & 27 & 2 & 50 \\
\hline Nylons & Vacuum & 27 & 3 & 24 \\
\hline Nylons & Vacuum & 27 & 4 & 46 \\
\hline Nylons & Vacuum & 27 & 5 & 20 \\
\hline Nylons & Vacuum & 27 & 6 & 18 \\
\hline Nylons & Vacuum & 27 & 7 & 46 \\
\hline Nylons & Vacuum & 27 & 8 & 21 \\
\hline Nylons & Vacuum & 27 & 9 & 42 \\
\hline Nylons & Vacuum & 27 & 10 & 18 \\
\hline Nylons & Vacuum & 27 & 11 & 11 \\
\hline Nylons & Vacuum & 27 & 12 & 17 \\
\hline Nylons & Vacuum & 27 & 13 & 18 \\
\hline Nylons & Vacuum & 27 & 14 & 24 \\
\hline Nylons & Vacuum & 27 & 15 & 18 \\
\hline Nylons & Vacuum & 28 & 1 & 25 \\
\hline Nylons & Vacuum & 28 & 2 & 33 \\
\hline Nylons & Vacuum & 28 & 3 & 26 \\
\hline Nylons & Vacuum & 28 & 4 & 39 \\
\hline Nylons & Vacuum & 28 & 5 & 34 \\
\hline Nylons & Vacuum & 28 & 6 & 19 \\
\hline
\end{tabular}




\begin{tabular}{|c|c|c|c|c|}
\hline Nylons & Vacuum & 28 & 7 & 25 \\
\hline Nylons & Vacuum & 28 & 8 & 21 \\
\hline Nylons & Vacuum & 28 & 9 & 30 \\
\hline Nylons & Vacuum & 28 & 10 & 24 \\
\hline Nylons & Vacuum & 28 & 11 & 18 \\
\hline Nylons & Vacuum & 29 & 1 & 30 \\
\hline Nylons & Vacuum & 29 & 2 & 30 \\
\hline Nylons & Vacuum & 29 & 3 & 14 \\
\hline Nylons & Vacuum & 29 & 4 & 34 \\
\hline Nylons & Vacuum & 29 & 5 & 30 \\
\hline Nylons & Vacuum & 29 & 6 & 5 \\
\hline Nylons & Vacuum & 29 & 7 & 46 \\
\hline Nylons & Vacuum & 29 & 8 & 19 \\
\hline Nylons & Vacuum & 29 & 9 & 33 \\
\hline Nylons & Vacuum & 29 & 10 & 39 \\
\hline Nylons & Vacuum & 30 & 1 & 35 \\
\hline Nylons & Vacuum & 30 & 2 & 34 \\
\hline Nylons & Vacuum & 30 & 3 & 21 \\
\hline Nylons & Vacuum & 30 & 4 & 22 \\
\hline Nylons & Vacuum & 30 & 5 & 14 \\
\hline Nylons & Vacuum & 30 & 6 & 34 \\
\hline Nylons & Vacuum & 30 & 7 & 16 \\
\hline Nylons & Vacuum & 30 & 8 & 22 \\
\hline Nylons & Vacuum & 30 & 9 & 22 \\
\hline Nylons & Vacuum & 30 & 10 & 28 \\
\hline Nylons & Vacuum & 31 & 1 & 31 \\
\hline Nylons & Vacuum & 31 & 2 & 45 \\
\hline Nylons & Vacuum & 31 & 3 & 41 \\
\hline Nylons & Vacuum & 31 & 4 & 11 \\
\hline Nylons & Vacuum & 31 & 5 & 32 \\
\hline Nylons & Vacuum & 31 & 6 & 5 \\
\hline Nylons & Vacuum & 31 & 7 & 26 \\
\hline Nylons & Vacuum & 31 & 8 & 4 \\
\hline Nylons & Vacuum & 31 & 9 & 25 \\
\hline Nylons & Vacuum & 32 & 1 & 13 \\
\hline Nylons & Vacuum & 32 & 2 & 18 \\
\hline Nylons & Vacuum & 32 & 3 & 24 \\
\hline Nylons & Vacuum & 32 & 4 & 26 \\
\hline Nylons & Vacuum & 32 & 5 & 26 \\
\hline Nylons & Vacuum & 32 & 6 & 20 \\
\hline Nylons & Vacuum & 32 & 7 & 13 \\
\hline Nylons & Vacuum & 32 & 8 & 22 \\
\hline Nylons & Vacuum & 32 & 9 & 47 \\
\hline Nylons & Vacuum & 32 & 10 & 25 \\
\hline Nylons & Vacuum & 33 & 1 & 46 \\
\hline
\end{tabular}




\begin{tabular}{|c|c|c|c|c|}
\hline Nylons & Vacuum & 33 & 2 & 50 \\
\hline Nylons & Vacuum & 33 & 3 & 45 \\
\hline Nylons & Vacuum & 33 & 4 & 28 \\
\hline Nylons & Vacuum & 33 & 5 & 34 \\
\hline Nylons & Vacuum & 33 & 6 & 38 \\
\hline Nylons & Vacuum & 33 & 7 & 41 \\
\hline Nylons & Vacuum & 33 & 8 & 40 \\
\hline Nylons & Vacuum & 34 & 1 & 27 \\
\hline Nylons & Vacuum & 34 & 2 & 38 \\
\hline Nylons & Vacuum & 34 & 3 & 36 \\
\hline Nylons & Vacuum & 34 & 4 & 26 \\
\hline Nylons & Vacuum & 34 & 5 & 34 \\
\hline Nylons & Vacuum & 34 & 6 & 23 \\
\hline Nylons & Vacuum & 34 & 7 & 36 \\
\hline Nylons & Vacuum & 34 & 8 & 39 \\
\hline Nylons & Vacuum & 34 & 9 & 39 \\
\hline Nylons & Vacuum & 34 & 10 & 53 \\
\hline Nylons & Vacuum & 34 & 11 & 30 \\
\hline Nylons & Vacuum & 35 & 1 & 26 \\
\hline Nylons & Vacuum & 35 & 2 & 30 \\
\hline Nylons & Vacuum & 35 & 3 & 19 \\
\hline Nylons & Vacuum & 35 & 4 & 47 \\
\hline Nylons & Vacuum & 35 & 5 & 43 \\
\hline Nylons & Vacuum & 35 & 6 & 29 \\
\hline Nylons & Vacuum & 35 & 7 & 35 \\
\hline Nylons & Vacuum & 35 & 8 & 39 \\
\hline Nylons & Vacuum & 35 & 9 & 43 \\
\hline Nylons & Vacuum & 35 & 10 & 35 \\
\hline Nylons & Vacuum & 35 & 11 & 35 \\
\hline Nylons & Vacuum & 35 & 12 & 39 \\
\hline Tennis_Shoes & TapeLift & 1 & 1 & 233 \\
\hline Tennis_Shoes & TapeLift & 1 & 2 & 74 \\
\hline Tennis_Shoes & TapeLift & 1 & 3 & 46 \\
\hline Tennis_Shoes & TapeLift & 1 & 4 & 51 \\
\hline Tennis_Shoes & TapeLift & 1 & 5 & 191 \\
\hline Tennis_Shoes & TapeLift & 1 & 6 & 70 \\
\hline Tennis_Shoes & TapeLift & 1 & 7 & 99 \\
\hline Tennis_Shoes & TapeLift & 1 & 8 & 61 \\
\hline Tennis_Shoes & TapeLift & 1 & 9 & 13 \\
\hline Tennis_Shoes & TapeLift & 1 & 10 & 58 \\
\hline Tennis_Shoes & TapeLift & 1 & 11 & 140 \\
\hline Tennis_Shoes & TapeLift & 1 & 12 & 65 \\
\hline Tennis_Shoes & TapeLift & 1 & 13 & 146 \\
\hline Tennis_Shoes & TapeLift & 1 & 14 & 49 \\
\hline Tennis_Shoes & TapeLift & 1 & 15 & 64 \\
\hline
\end{tabular}




\begin{tabular}{|c|c|}
\hline Tennis_Shoes & TapeLift \\
\hline Tennis_Shoes & TapeLift \\
\hline Tennis_Shoes & TapeLift \\
\hline Tennis_Shoes & TapeLift \\
\hline Tennis_Shoes & TapeLift \\
\hline Tennis_Shoes & TapeLift \\
\hline Tennis_Shoes & TapeLift \\
\hline Tennis_Shoes & TapeLift \\
\hline Tennis_Shoes & TapeLift \\
\hline Tennis_Shoes & TapeLift \\
\hline Tennis_Shoes & TapeLift \\
\hline Tennis_Shoes & TapeLi \\
\hline Tennis_Shoes & TapeLift \\
\hline Tennis_Shoes & TapeLift \\
\hline Tennis_Shoes & TapeLift \\
\hline Tennis_Shoes & TapeLift \\
\hline Tennis_Shoes & TapeLift \\
\hline Tennis_Shoes & TapeLift \\
\hline Tennis_Shoes & TapeLift \\
\hline Tennis_Shoes & TapeLift \\
\hline Tennis_Shoes & TapeLift \\
\hline Tennis_Shoes & TapeLift \\
\hline Tennis_Shoes & TapeLift \\
\hline Tennis_Shoes & TapeLift \\
\hline Tennis_Shoes & TapeLift \\
\hline Tennis_Shoes & TapeLift \\
\hline Tennis_Shoes & TapeLift \\
\hline Tennis_Shoes & TapeLift \\
\hline Tennis_Shoes & TapeLift \\
\hline Tennis_Shoes & TapeLift \\
\hline Tennis_Shoes & TapeLift \\
\hline Tennis_Shoes & TapeLift \\
\hline Tennis_Shoes & TapeLift \\
\hline Tennis_Shoes & TapeLift \\
\hline Tennis_Shoes & TapeLift \\
\hline Tennis_Shoes & TapeLift \\
\hline Tennis_Shoes & TapeLift \\
\hline Tennis_Shoes & TapeLift \\
\hline Tennis_Shoes & TapeLift \\
\hline Tennis_Shoes & TapeLift \\
\hline Tennis_Shoes & TapeLift \\
\hline Tennis_Shoes & TapeLift \\
\hline Tennis_Shoes & TapeLift \\
\hline Tennis_Shoes & TapeLift \\
\hline Tennis_Shoes & TapeLift \\
\hline
\end{tabular}

\begin{tabular}{|c|c|}
\hline 1 & 16 \\
\hline 1 & 17 \\
\hline 1 & 18 \\
\hline 2 & 1 \\
\hline 2 & 2 \\
\hline 2 & 3 \\
\hline 2 & 4 \\
\hline 2 & 5 \\
\hline 2 & 6 \\
\hline 2 & 7 \\
\hline 3 & 1 \\
\hline 3 & 2 \\
\hline 3 & 3 \\
\hline 3 & 4 \\
\hline 3 & 5 \\
\hline 3 & 6 \\
\hline 3 & 7 \\
\hline 3 & 8 \\
\hline 3 & 9 \\
\hline 3 & 10 \\
\hline 3 & 11 \\
\hline 3 & 12 \\
\hline 3 & 13 \\
\hline 3 & 14 \\
\hline 3 & 15 \\
\hline 4 & 1 \\
\hline 4 & 2 \\
\hline 4 & 3 \\
\hline 4 & 4 \\
\hline 4 & 5 \\
\hline 4 & 6 \\
\hline 4 & 7 \\
\hline 4 & 8 \\
\hline 4 & 9 \\
\hline 4 & 10 \\
\hline 4 & 11 \\
\hline 4 & 12 \\
\hline 4 & 13 \\
\hline 4 & 14 \\
\hline 4 & 15 \\
\hline 4 & 16 \\
\hline 4 & 17 \\
\hline 4 & 18 \\
\hline 4 & 19 \\
\hline 4 & 20 \\
\hline
\end{tabular}




\begin{tabular}{|c|c|}
\hline Tennis_Shoes & TapeLift \\
\hline Tennis_Shoes & TapeLift \\
\hline Tennis_Shoes & TapeLift \\
\hline Tennis_Shoes & TapeLift \\
\hline Tennis_Shoes & TapeLift \\
\hline Tennis_Shoes & TapeLift \\
\hline Tennis_Shoes & TapeLift \\
\hline Tennis_Shoes & TapeLift \\
\hline Tennis_Shoes & TapeLift \\
\hline Tennis_Shoes & TapeLift \\
\hline Tennis_Shoes & TapeLift \\
\hline Tennis_Shoes & TapeLift \\
\hline Tennis_Shoes & TapeLift \\
\hline Tennis_Shoes & TapeLift \\
\hline Tennis_Shoes & TapeLift \\
\hline Tennis_Shoes & TapeLift \\
\hline Tennis_Shoes & TapeLift \\
\hline Tennis_Shoes & TapeLift \\
\hline Tennis_Shoes & TapeLift \\
\hline Tennis_Shoes & TapeLift \\
\hline Tennis_Shoes & TapeLift \\
\hline Tennis_Shoes & TapeLift \\
\hline Tennis_Shoes & TapeLift \\
\hline Tennis_Shoes & TapeLift \\
\hline Tennis_Shoes & TapeLift \\
\hline Tennis_Shoes & TapeLift \\
\hline Tennis_Shoes & TapeLift \\
\hline Tennis_Shoes & TapeLift \\
\hline Tennis_Shoes & TapeLift \\
\hline Tennis_Shoes & TapeLift \\
\hline Tennis_Shoes & TapeLift \\
\hline Tennis_Shoes & TapeLift \\
\hline Tennis_Shoes & TapeLift \\
\hline Tennis_Shoes & TapeLift \\
\hline Tennis_Shoes & TapeLift \\
\hline Tennis_Shoes & TapeLift \\
\hline Tennis_Shoes & TapeLift \\
\hline Tennis_Shoes & TapeLift \\
\hline Tennis_Shoes & TapeLift \\
\hline Tennis_Shoes & TapeLift \\
\hline Tennis_Shoes & TapeLift \\
\hline Tennis_Shoes & TapeLift \\
\hline Tennis_Shoes & TapeLift \\
\hline Tennis_Shoes & TapeLi \\
\hline Tennis_Shoes & TapeLift \\
\hline
\end{tabular}

\begin{tabular}{|c|c|}
\hline 4 & 21 \\
\hline 4 & 22 \\
\hline 4 & 23 \\
\hline 4 & 24 \\
\hline 5 & 1 \\
\hline 5 & 2 \\
\hline 5 & 3 \\
\hline 5 & 4 \\
\hline 5 & 5 \\
\hline 5 & 6 \\
\hline 5 & 7 \\
\hline 5 & 8 \\
\hline 5 & 9 \\
\hline 5 & 10 \\
\hline 5 & 11 \\
\hline 5 & 12 \\
\hline 5 & 13 \\
\hline 5 & 14 \\
\hline 5 & 15 \\
\hline 5 & 16 \\
\hline 6 & 1 \\
\hline 6 & 2 \\
\hline 6 & 3 \\
\hline 6 & 4 \\
\hline 6 & 5 \\
\hline 6 & 6 \\
\hline 6 & 7 \\
\hline 6 & 8 \\
\hline 6 & 9 \\
\hline 6 & 10 \\
\hline 6 & 11 \\
\hline 6 & 12 \\
\hline 6 & 13 \\
\hline 6 & 14 \\
\hline 6 & 15 \\
\hline 6 & 16 \\
\hline 6 & 17 \\
\hline 6 & 18 \\
\hline 6 & 19 \\
\hline 6 & 20 \\
\hline 7 & 1 \\
\hline 7 & 2 \\
\hline 7 & 3 \\
\hline 7 & 4 \\
\hline 7 & 5 \\
\hline
\end{tabular}




\begin{tabular}{|c|c|c|c|c|}
\hline Tennis_Shoes & TapeLift & 7 & 6 & 21 \\
\hline Tennis_Shoes & TapeLift & 7 & 7 & 29 \\
\hline Tennis_Shoes & TapeLift & 7 & 8 & 69 \\
\hline Tennis_Shoes & TapeLift & 7 & 9 & 75 \\
\hline Tennis_Shoes & TapeLift & 7 & 10 & 37 \\
\hline Tennis_Shoes & TapeLift & 7 & 11 & 36 \\
\hline Tennis_Shoes & TapeLift & 7 & 12 & 34 \\
\hline Tennis_Shoes & TapeLift & 7 & 13 & 36 \\
\hline Tennis_Shoes & TapeLift & 7 & 14 & 45 \\
\hline Tennis_Shoes & TapeLift & 7 & 15 & 51 \\
\hline Tennis_Shoes & TapeLift & 7 & 16 & 43 \\
\hline Tennis_Shoes & TapeLift & 7 & 17 & 24 \\
\hline Tennis_Shoes & TapeLift & 7 & 18 & 92 \\
\hline Tennis_Shoes & TapeLift & 7 & 19 & 26 \\
\hline Tennis_Shoes & TapeLift & 7 & 20 & 87 \\
\hline Tennis_Shoes & TapeLift & 8 & 1 & 36 \\
\hline Tennis_Shoes & TapeLift & 8 & 2 & 37 \\
\hline Tennis_Shoes & TapeLift & 8 & 3 & 52 \\
\hline Tennis_Shoes & TapeLift & 8 & 4 & 74 \\
\hline Tennis_Shoes & TapeLift & 8 & 5 & 35 \\
\hline Tennis_Shoes & TapeLift & 8 & 6 & 24 \\
\hline Tennis_Shoes & TapeLift & 8 & 7 & 28 \\
\hline Tennis_Shoes & TapeLift & 8 & 8 & 56 \\
\hline Tennis_Shoes & TapeLift & 8 & 9 & 93 \\
\hline Tennis_Shoes & TapeLift & 8 & 10 & 52 \\
\hline Tennis_Shoes & TapeLift & 8 & 11 & 39 \\
\hline Tennis_Shoes & TapeLift & 8 & 12 & 62 \\
\hline Tennis_Shoes & TapeLift & 8 & 13 & 48 \\
\hline Tennis_Shoes & TapeLift & 8 & 14 & 15 \\
\hline Tennis_Shoes & TapeLift & 8 & 15 & 45 \\
\hline Tennis_Shoes & TapeLift & 8 & 16 & 56 \\
\hline Tennis_Shoes & TapeLift & 8 & 17 & 32 \\
\hline Tennis_Shoes & TapeLift & 8 & 18 & 51 \\
\hline Tennis_Shoes & TapeLift & 8 & 19 & 37 \\
\hline Tennis_Shoes & TapeLift & 8 & 20 & 31 \\
\hline Tennis_Shoes & TapeLift & 8 & 21 & 53 \\
\hline Tennis_Shoes & TapeLift & 8 & 22 & 42 \\
\hline Tennis_Shoes & TapeLift & 8 & 23 & 28 \\
\hline Tennis_Shoes & TapeLift & 8 & 24 & 29 \\
\hline Tennis_Shoes & TapeLift & 9 & 1 & 53 \\
\hline Tennis_Shoes & TapeLift & 9 & 2 & 27 \\
\hline Tennis_Shoes & TapeLift & 9 & 3 & 5 \\
\hline Tennis_Shoes & TapeLift & 9 & 4 & 61 \\
\hline Tennis_Shoes & TapeLift & 9 & 5 & 60 \\
\hline Tennis_Shoes & TapeLift & 9 & 6 & 52 \\
\hline
\end{tabular}




\begin{tabular}{|c|c|}
\hline Tennis_Shoes & TapeLift \\
\hline Tennis_Shoes & TapeLift \\
\hline Tennis_Shoes & TapeLift \\
\hline Tennis_Shoes & TapeLift \\
\hline Tennis_Shoes & TapeLift \\
\hline Tennis_Shoes & TapeLift \\
\hline Tennis_Shoes & TapeLift \\
\hline Tennis_Shoes & TapeLift \\
\hline Tennis_Shoes & TapeLift \\
\hline Tennis_Shoes & TapeLift \\
\hline Tennis_Shoes & TapeLift \\
\hline Tennis_Shoes & TapeLift \\
\hline Tennis_Shoes & TapeLift \\
\hline Tennis_Shoes & TapeLift \\
\hline Tennis_Shoes & TapeLift \\
\hline Tennis_Shoes & TapeLift \\
\hline Tennis_Shoes & TapeLift \\
\hline Tennis_Shoes & TapeLift \\
\hline Tennis_Shoes & TapeLift \\
\hline Tennis_Shoes & TapeLift \\
\hline Tennis_Shoes & TapeLift \\
\hline Tennis_Shoes & TapeLift \\
\hline Tennis_Shoes & TapeLift \\
\hline Tennis_Shoes & TapeLift \\
\hline Tennis_Shoes & TapeLift \\
\hline Tennis_Shoes & TapeLift \\
\hline Tennis_Shoes & TapeLift \\
\hline Tennis_Shoes & TapeLift \\
\hline Tennis_Shoes & TapeLift \\
\hline Tennis_Shoes & TapeLift \\
\hline Tennis_Shoes & TapeLift \\
\hline Tennis_Shoes & TapeLift \\
\hline Tennis_Shoes & TapeLift \\
\hline Tennis_Shoes & TapeLift \\
\hline Tennis_Shoes & TapeLift \\
\hline Tennis_Shoes & TapeLift \\
\hline Tennis_Shoes & TapeLift \\
\hline Tennis_Shoes & TapeLift \\
\hline Tennis_Shoes & TapeLift \\
\hline Tennis_Shoes & TapeLift \\
\hline Tennis_Shoes & TapeLift \\
\hline Tennis_Shoes & TapeLift \\
\hline Tennis_Shoes & TapeLift \\
\hline Tennis_Shoes & TapeLi \\
\hline Tennis_Shoes & TapeLift \\
\hline
\end{tabular}

\begin{tabular}{|c|c|c|}
\hline 9 & 7 & 31 \\
\hline 9 & 8 & 78 \\
\hline 9 & 9 & 24 \\
\hline 9 & 10 & 72 \\
\hline 9 & 11 & 97 \\
\hline 9 & 12 & 54 \\
\hline 9 & 13 & 28 \\
\hline 9 & 14 & 83 \\
\hline 9 & 15 & 70 \\
\hline 9 & 16 & 46 \\
\hline 9 & 17 & 50 \\
\hline 9 & 18 & 13 \\
\hline 9 & 19 & 39 \\
\hline 9 & 20 & 24 \\
\hline 10 & 1 & 31 \\
\hline 10 & 2 & 52 \\
\hline 10 & 3 & 29 \\
\hline 10 & 4 & 25 \\
\hline 10 & 5 & 43 \\
\hline 10 & 6 & 78 \\
\hline 10 & 7 & 44 \\
\hline 10 & 8 & 48 \\
\hline 10 & 9 & 67 \\
\hline 10 & 10 & 93 \\
\hline 10 & 11 & 64 \\
\hline 10 & 12 & 36 \\
\hline 10 & 13 & 15 \\
\hline 10 & 14 & 12 \\
\hline 10 & 15 & 28 \\
\hline 10 & 16 & 30 \\
\hline 10 & 17 & 19 \\
\hline 10 & 18 & 30 \\
\hline 10 & 19 & 45 \\
\hline 10 & 20 & 69 \\
\hline 10 & 21 & 70 \\
\hline 10 & 22 & 26 \\
\hline 10 & 23 & 31 \\
\hline 10 & 24 & 16 \\
\hline 11 & 1 & 63 \\
\hline 11 & 2 & 22 \\
\hline 11 & 3 & 44 \\
\hline 11 & 4 & 66 \\
\hline 11 & 5 & 113 \\
\hline 11 & 6 & 76 \\
\hline 11 & 7 & 40 \\
\hline
\end{tabular}




\begin{tabular}{|c|c|}
\hline Tennis_Shoes & TapeLift \\
\hline Tennis_Shoes & TapeLift \\
\hline Tennis_Shoes & TapeLift \\
\hline Tennis_Shoes & TapeLift \\
\hline Tennis_Shoes & TapeLift \\
\hline Tennis_Shoes & TapeLift \\
\hline Tennis_Shoes & TapeLift \\
\hline Tennis_Shoes & TapeLift \\
\hline Tennis_Shoes & TapeLift \\
\hline Tennis_Shoes & TapeLift \\
\hline Tennis_Shoes & TapeLift \\
\hline Tennis_Shoes & TapeLift \\
\hline Tennis_Shoes & TapeLift \\
\hline Tennis_Shoes & TapeLift \\
\hline Tennis_Shoes & TapeLift \\
\hline Tennis_Shoes & TapeLift \\
\hline Tennis_Shoes & TapeLift \\
\hline Tennis_Shoes & TapeLift \\
\hline Tennis_Shoes & TapeLift \\
\hline Tennis_Shoes & TapeLift \\
\hline Tennis_Shoes & TapeLift \\
\hline Tennis_Shoes & TapeLift \\
\hline Tennis_Shoes & TapeLift \\
\hline Tennis_Shoes & TapeLift \\
\hline Tennis_Shoes & TapeLift \\
\hline Tennis_Shoes & TapeLift \\
\hline Tennis_Shoes & TapeLift \\
\hline Tennis_Shoes & TapeLift \\
\hline Tennis_Shoes & TapeLift \\
\hline Tennis_Shoes & TapeLift \\
\hline Tennis_Shoes & TapeLift \\
\hline Tennis_Shoes & TapeLift \\
\hline Tennis_Shoes & TapeLift \\
\hline Tennis_Shoes & TapeLift \\
\hline Tennis_Shoes & TapeLift \\
\hline Tennis_Shoes & TapeLift \\
\hline Tennis_Shoes & TapeLift \\
\hline Tennis_Shoes & TapeLift \\
\hline Tennis_Shoes & TapeLift \\
\hline Tennis_Shoes & TapeLift \\
\hline Tennis_Shoes & TapeLift \\
\hline Tennis_Shoes & TapeLift \\
\hline Tennis_Shoes & TapeLift \\
\hline Tennis_Shoes & TapeLi \\
\hline Tennis_Shoes & TapeLift \\
\hline
\end{tabular}

$\begin{array}{ll}8 & 18\end{array}$

$9 \quad 138$

$10 \quad 164$

$11 \quad 57$

$12 \quad 31$

$13 \quad 28$

$14 \quad 75$

$15 \quad 56$

$16 \quad 23$

$17 \quad 41$

$18 \quad 48$

$19 \quad 29$

$20 \quad 33$

$21 \quad 22$

$22 \quad 41$

$23 \quad 32$

$24 \quad 81$

$25 \quad 120$

148

255

$3 \quad 28$

441

562

638

$\begin{array}{ll}7 & 19\end{array}$

845

940

$10 \quad 111$

1140

$12 \quad 37$

1343

$14 \quad 40$

$15 \quad 30$

$16 \quad 19$

$17 \quad 27$

$18 \quad 43$

$19 \quad 55$

$20 \quad 19$

$21 \quad 52$

$22 \quad 42$

131

248

$3 \quad 32$

$4 \quad 34$

$5 \quad 71$ 


\begin{tabular}{|c|c|c|c|}
\hline Tennis_Shoes & TapeLift & 13 & 6 \\
\hline Tennis_Shoes & TapeLift & 13 & 7 \\
\hline Tennis_Shoes & TapeLift & 13 & 8 \\
\hline Tennis_Shoes & TapeLift & 13 & 9 \\
\hline Tennis_Shoes & TapeLift & 13 & 10 \\
\hline Tennis_Shoes & TapeLift & 13 & 11 \\
\hline Tennis_Shoes & TapeLift & 13 & 12 \\
\hline Tennis_Shoes & TapeLift & 13 & 13 \\
\hline Tennis_Shoes & TapeLift & 13 & 14 \\
\hline Tennis_Shoes & TapeLift & 13 & 15 \\
\hline Tennis_Shoes & TapeLift & 13 & 16 \\
\hline Tennis_Shoes & TapeLift & 13 & 17 \\
\hline Tennis_Shoes & TapeLift & 13 & 18 \\
\hline Tennis_Shoes & TapeLift & 13 & 19 \\
\hline Tennis_Shoes & TapeLift & 13 & 20 \\
\hline Tennis_Shoes & TapeLift & 13 & 21 \\
\hline Tennis_Shoes & TapeLift & 13 & 22 \\
\hline Tennis_Shoes & TapeLift & 13 & 23 \\
\hline Tennis_Shoes & TapeLift & 13 & 24 \\
\hline Tennis_Shoes & TapeLift & 13 & 25 \\
\hline Tennis_Shoes & TapeLift & 14 & 1 \\
\hline Tennis_Shoes & TapeLift & 14 & 2 \\
\hline Tennis_Shoes & TapeLift & 14 & 3 \\
\hline Tennis_Shoes & TapeLift & 14 & 4 \\
\hline Tennis_Shoes & TapeLift & 14 & 5 \\
\hline Tennis_Shoes & TapeLift & 14 & 6 \\
\hline Tennis_Shoes & TapeLift & 14 & 7 \\
\hline Tennis_Shoes & TapeLift & 14 & 8 \\
\hline Tennis_Shoes & TapeLift & 14 & 9 \\
\hline Tennis_Shoes & TapeLift & 14 & 10 \\
\hline Tennis_Shoes & TapeLift & 14 & 11 \\
\hline Tennis_Shoes & TapeLift & 14 & 12 \\
\hline Tennis_Shoes & TapeLift & 14 & 13 \\
\hline Tennis_Shoes & TapeLift & 14 & 14 \\
\hline Tennis_Shoes & TapeLift & 14 & 15 \\
\hline Tennis_Shoes & TapeLift & 14 & 16 \\
\hline Tennis_Shoes & TapeLift & 14 & 17 \\
\hline Tennis_Shoes & TapeLift & 14 & 18 \\
\hline Tennis_Shoes & TapeLift & 14 & 19 \\
\hline Tennis_Shoes & TapeLift & 14 & 20 \\
\hline Tennis_Shoes & TapeLift & 14 & 21 \\
\hline Tennis_Shoes & TapeLift & 14 & 22 \\
\hline Tennis_Shoes & TapeLift & 14 & 23 \\
\hline Tennis_Shoes & TapeLift & 14 & 24 \\
\hline Tennis_Shoes & TapeLift & 14 & 25 \\
\hline
\end{tabular}




\begin{tabular}{|c|c|c|c|}
\hline Tennis_Shoes & TapeLift & 14 & 26 \\
\hline Tennis_Shoes & TapeLift & 14 & 27 \\
\hline Tennis_Shoes & TapeLift & 14 & 28 \\
\hline Tennis_Shoes & TapeLift & 14 & 29 \\
\hline Tennis_Shoes & TapeLift & 14 & 30 \\
\hline Tennis_Shoes & TapeLift & 14 & 31 \\
\hline Tennis_Shoes & TapeLift & 14 & 32 \\
\hline Tennis_Shoes & TapeLift & 14 & 33 \\
\hline Tennis_Shoes & TapeLift & 15 & 1 \\
\hline Tennis_Shoes & TapeLift & 15 & 2 \\
\hline Tennis_Shoes & TapeLift & 15 & 3 \\
\hline Tennis_Shoes & TapeLift & 15 & 4 \\
\hline Tennis_Shoes & TapeLift & 15 & 5 \\
\hline Tennis_Shoes & TapeLift & 15 & 6 \\
\hline Tennis_Shoes & TapeLift & 15 & 7 \\
\hline Tennis_Shoes & TapeLift & 15 & 8 \\
\hline Tennis_Shoes & TapeLift & 15 & 9 \\
\hline Tennis_Shoes & TapeLift & 15 & 10 \\
\hline Tennis_Shoes & TapeLift & 15 & 11 \\
\hline Tennis_Shoes & TapeLift & 15 & 12 \\
\hline Tennis_Shoes & TapeLift & 15 & 13 \\
\hline Tennis_Shoes & TapeLift & 15 & 14 \\
\hline Tennis_Shoes & TapeLift & 15 & 15 \\
\hline Tennis_Shoes & TapeLift & 15 & 16 \\
\hline Tennis_Shoes & TapeLift & 15 & 17 \\
\hline Tennis_Shoes & TapeLift & 15 & 18 \\
\hline Tennis_Shoes & TapeLift & 15 & 19 \\
\hline Tennis_Shoes & TapeLift & 15 & 20 \\
\hline Tennis_Shoes & TapeLift & 15 & 21 \\
\hline Tennis_Shoes & TapeLift & 15 & 22 \\
\hline Tennis_Shoes & TapeLift & 15 & 23 \\
\hline Tennis_Shoes & TapeLift & 15 & 24 \\
\hline Tennis_Shoes & TapeLift & 15 & 25 \\
\hline Tennis_Shoes & TapeLift & 16 & 1 \\
\hline Tennis_Shoes & TapeLift & 16 & 2 \\
\hline Tennis_Shoes & TapeLift & 16 & 3 \\
\hline Tennis_Shoes & TapeLift & 16 & 4 \\
\hline Tennis_Shoes & TapeLift & 16 & 5 \\
\hline Tennis_Shoes & TapeLift & 16 & 6 \\
\hline Tennis_Shoes & TapeLift & 16 & 7 \\
\hline Tennis_Shoes & TapeLift & 16 & 8 \\
\hline Tennis_Shoes & TapeLift & 16 & 9 \\
\hline Tennis_Shoes & TapeLift & 16 & 10 \\
\hline Tennis_Shoes & TapeLift & 16 & 11 \\
\hline Tennis_Shoes & TapeLift & 16 & 12 \\
\hline
\end{tabular}




$\begin{array}{lll}16 & 16 & 185\end{array}$

$\begin{array}{lll}16 & 17 & 51\end{array}$

$\begin{array}{lll}16 & 18 & 53\end{array}$

$\begin{array}{lll}16 & 19 & 38\end{array}$

$16 \quad 20 \quad 70$

$\begin{array}{lll}16 & 21 & 57\end{array}$

$16 \quad 22 \quad 58$

$\begin{array}{lll}16 & 23 & 107\end{array}$

$\begin{array}{lll}17 & 1 & 103\end{array}$

$\begin{array}{lll}17 & 2 & 73\end{array}$

$\begin{array}{lll}17 & 3 & 129\end{array}$

$\begin{array}{lll}17 & 4 & 68\end{array}$

$\begin{array}{lll}17 & 5 & 81\end{array}$

$\begin{array}{lll}17 & 6 & 129\end{array}$

$\begin{array}{lll}17 & 7 & 42\end{array}$

$\begin{array}{lll}17 & 8 & 49\end{array}$

$\begin{array}{lll}17 & 9 & 63\end{array}$

$\begin{array}{lll}17 & 10 & 45\end{array}$

$\begin{array}{lll}17 & 11 & 43\end{array}$

$17 \quad 12 \quad 58$

$\begin{array}{lll}17 & 13 & 65\end{array}$

$\begin{array}{lll}17 & 14 & 71\end{array}$

$\begin{array}{lll}17 & 15 & 47\end{array}$

$\begin{array}{lll}17 & 16 & 56\end{array}$

$\begin{array}{lll}17 & 17 & 47\end{array}$

$\begin{array}{lll}17 & 18 & 57\end{array}$

$\begin{array}{lll}17 & 19 & 34\end{array}$

$\begin{array}{lll}17 & 20 & 82\end{array}$

$18 \quad 1 \quad 84$

$18 \quad 2 \quad 93$

$\begin{array}{lll}18 & 3 & 58\end{array}$

$\begin{array}{lll}18 & 4 & 74\end{array}$

$18 \quad 5 \quad 146$

$\begin{array}{lll}18 & 6 & 57\end{array}$

$\begin{array}{lll}18 & 7 & 59\end{array}$

$18 \quad 8 \quad 49$

$\begin{array}{lll}18 & 9 & 49\end{array}$

$\begin{array}{lll}18 & 10 & 86\end{array}$

$\begin{array}{lll}18 & 11 & 72\end{array}$

$18 \quad 12 \quad 42$

$18 \quad 13 \quad 26$

$\begin{array}{lll}18 & 14 & 63\end{array}$




\begin{tabular}{|c|c|c|c|}
\hline Tennis_Shoes & TapeLift & 18 & 15 \\
\hline Tennis_Shoes & TapeLift & 18 & 16 \\
\hline Tennis_Shoes & TapeLift & 18 & 17 \\
\hline Tennis_Shoes & TapeLift & 18 & 18 \\
\hline Tennis_Shoes & TapeLift & 18 & 19 \\
\hline Tennis_Shoes & TapeLift & 18 & 20 \\
\hline Tennis_Shoes & TapeLift & 18 & 21 \\
\hline Tennis_Shoes & TapeLift & 18 & 22 \\
\hline Tennis_Shoes & TapeLift & 19 & 1 \\
\hline Tennis_Shoes & TapeLift & 19 & 2 \\
\hline Tennis_Shoes & TapeLift & 19 & 3 \\
\hline Tennis_Shoes & TapeLift & 19 & 4 \\
\hline Tennis_Shoes & TapeLift & 19 & 5 \\
\hline Tennis_Shoes & TapeLift & 19 & 6 \\
\hline Tennis_Shoes & TapeLift & 19 & 7 \\
\hline Tennis_Shoes & TapeLift & 19 & 8 \\
\hline Tennis_Shoes & TapeLift & 19 & 9 \\
\hline Tennis_Shoes & TapeLift & 19 & 10 \\
\hline Tennis_Shoes & TapeLift & 19 & 11 \\
\hline Tennis_Shoes & TapeLift & 19 & 12 \\
\hline Tennis_Shoes & TapeLift & 19 & 13 \\
\hline Tennis_Shoes & TapeLift & 19 & 14 \\
\hline Tennis_Shoes & TapeLift & 19 & 15 \\
\hline Tennis_Shoes & TapeLift & 19 & 16 \\
\hline Tennis_Shoes & TapeLift & 19 & 17 \\
\hline Tennis_Shoes & TapeLift & 19 & 18 \\
\hline Tennis_Shoes & TapeLift & 19 & 19 \\
\hline Tennis_Shoes & TapeLift & 19 & 20 \\
\hline Tennis_Shoes & TapeLift & 20 & 1 \\
\hline Tennis_Shoes & TapeLift & 20 & 2 \\
\hline Tennis_Shoes & TapeLift & 20 & 3 \\
\hline Tennis_Shoes & TapeLift & 20 & 4 \\
\hline Tennis_Shoes & TapeLift & 20 & 5 \\
\hline Tennis_Shoes & TapeLift & 20 & 6 \\
\hline Tennis_Shoes & TapeLift & 20 & 7 \\
\hline Tennis_Shoes & TapeLift & 20 & 8 \\
\hline Tennis_Shoes & TapeLift & 20 & 9 \\
\hline Tennis_Shoes & TapeLift & 20 & 10 \\
\hline Tennis_Shoes & TapeLift & 20 & 11 \\
\hline Tennis_Shoes & TapeLift & 20 & 12 \\
\hline Tennis_Shoes & TapeLift & 20 & 13 \\
\hline Tennis_Shoes & TapeLift & 20 & 14 \\
\hline Tennis_Shoes & TapeLift & 20 & 15 \\
\hline Tennis_Shoes & TapeLift & 20 & 16 \\
\hline Tennis_Shoes & TapeLift & 20 & 17 \\
\hline
\end{tabular}




\begin{tabular}{|c|c|c|c|c|}
\hline Tennis_Shoes & TapeLift & 20 & 18 & 72 \\
\hline Tennis_Shoes & TapeLift & 20 & 19 & 56 \\
\hline Tennis_Shoes & TapeLift & 20 & 20 & 87 \\
\hline Tennis_Shoes & TapeLift & 20 & 21 & 57 \\
\hline Tennis_Shoes & TapeLift & 20 & 22 & 60 \\
\hline Tennis_Shoes & TapeLift & 20 & 23 & 53 \\
\hline Tennis_Shoes & TapeLift & 20 & 24 & 38 \\
\hline Tennis_Shoes & TapeLift & 20 & 25 & 37 \\
\hline Tennis_Shoes & TapeLift & 20 & 26 & 139 \\
\hline Tennis_Shoes & TapeLift & 20 & 27 & 59 \\
\hline Tennis_Shoes & TapeLift & 20 & 28 & 40 \\
\hline Tennis_Shoes & TapeLift & 20 & 29 & 105 \\
\hline Tennis_Shoes & TapeLift & 21 & 1 & 95 \\
\hline Tennis_Shoes & TapeLift & 21 & 2 & 58 \\
\hline Tennis_Shoes & TapeLift & 21 & 3 & 89 \\
\hline Tennis_Shoes & TapeLift & 21 & 4 & 52 \\
\hline Tennis_Shoes & TapeLift & 21 & 5 & 46 \\
\hline Tennis_Shoes & TapeLift & 21 & 6 & 31 \\
\hline Tennis_Shoes & TapeLift & 21 & 7 & 34 \\
\hline Tennis_Shoes & TapeLift & 21 & 8 & 15 \\
\hline Tennis_Shoes & TapeLift & 21 & 9 & 107 \\
\hline Tennis_Shoes & TapeLift & 21 & 10 & 108 \\
\hline Tennis_Shoes & TapeLift & 21 & 11 & 41 \\
\hline Tennis_Shoes & TapeLift & 21 & 12 & 19 \\
\hline Tennis_Shoes & TapeLift & 21 & 13 & 110 \\
\hline Tennis_Shoes & TapeLift & 21 & 14 & 79 \\
\hline Tennis_Shoes & TapeLift & 21 & 15 & 71 \\
\hline Tennis_Shoes & TapeLift & 21 & 16 & 37 \\
\hline Tennis_Shoes & TapeLift & 21 & 17 & 45 \\
\hline Tennis_Shoes & TapeLift & 21 & 18 & 22 \\
\hline Tennis_Shoes & TapeLift & 21 & 19 & 110 \\
\hline Tennis_Shoes & TapeLift & 21 & 20 & 56 \\
\hline Tennis_Shoes & TapeLift & 21 & 21 & 30 \\
\hline Tennis_Shoes & TapeLift & 21 & 22 & 52 \\
\hline Tennis_Shoes & TapeLift & 21 & 23 & 67 \\
\hline Tennis_Shoes & TapeLift & 21 & 24 & 61 \\
\hline Tennis_Shoes & TapeLift & 21 & 25 & 26 \\
\hline Tennis_Shoes & TapeLift & 22 & 1 & 65 \\
\hline Tennis_Shoes & TapeLift & 22 & 2 & 69 \\
\hline Tennis_Shoes & TapeLift & 22 & 3 & 44 \\
\hline Tennis_Shoes & TapeLift & 22 & 4 & 98 \\
\hline Tennis_Shoes & TapeLift & 22 & 5 & 66 \\
\hline Tennis_Shoes & TapeLift & 22 & 6 & 41 \\
\hline Tennis_Shoes & TapeLift & 22 & 7 & 52 \\
\hline Tennis_Shoes & TapeLift & 22 & 8 & 14 \\
\hline
\end{tabular}




\begin{tabular}{|c|c|c|c|}
\hline Tennis_Shoes & TapeLift & 22 & 9 \\
\hline Tennis_Shoes & TapeLift & 22 & 10 \\
\hline Tennis_Shoes & TapeLift & 22 & 11 \\
\hline Tennis_Shoes & TapeLift & 22 & 12 \\
\hline Tennis_Shoes & TapeLift & 22 & 13 \\
\hline Tennis_Shoes & TapeLift & 22 & 14 \\
\hline Tennis_Shoes & TapeLift & 22 & 15 \\
\hline Tennis_Shoes & TapeLift & 22 & 16 \\
\hline Tennis_Shoes & TapeLift & 22 & 17 \\
\hline Tennis_Shoes & TapeLift & 22 & 18 \\
\hline Tennis_Shoes & TapeLift & 22 & 19 \\
\hline Tennis_Shoes & TapeLift & 22 & 20 \\
\hline Tennis_Shoes & TapeLift & 23 & 1 \\
\hline Tennis_Shoes & TapeLift & 23 & 2 \\
\hline Tennis_Shoes & TapeLift & 23 & 3 \\
\hline Tennis_Shoes & TapeLift & 23 & 4 \\
\hline Tennis_Shoes & TapeLift & 23 & 5 \\
\hline Tennis_Shoes & TapeLift & 23 & 6 \\
\hline Tennis_Shoes & TapeLift & 23 & 7 \\
\hline Tennis_Shoes & TapeLift & 23 & 8 \\
\hline Tennis_Shoes & TapeLift & 23 & 9 \\
\hline Tennis_Shoes & TapeLift & 23 & 10 \\
\hline Tennis_Shoes & TapeLift & 23 & 11 \\
\hline Tennis_Shoes & TapeLift & 23 & 12 \\
\hline Tennis_Shoes & TapeLift & 23 & 13 \\
\hline Tennis_Shoes & TapeLift & 23 & 14 \\
\hline Tennis_Shoes & TapeLift & 23 & 15 \\
\hline Tennis_Shoes & TapeLift & 23 & 16 \\
\hline Tennis_Shoes & TapeLift & 23 & 17 \\
\hline Tennis_Shoes & TapeLift & 23 & 18 \\
\hline Tennis_Shoes & TapeLift & 23 & 19 \\
\hline Tennis_Shoes & TapeLift & 23 & 20 \\
\hline Tennis_Shoes & TapeLift & 23 & 21 \\
\hline Tennis_Shoes & TapeLift & 23 & 22 \\
\hline Tennis_Shoes & TapeLift & 24 & 1 \\
\hline Tennis_Shoes & TapeLift & 24 & 2 \\
\hline Tennis_Shoes & TapeLift & 24 & 3 \\
\hline Tennis_Shoes & TapeLift & 24 & 4 \\
\hline Tennis_Shoes & TapeLift & 24 & 5 \\
\hline Tennis_Shoes & TapeLift & 24 & 6 \\
\hline Tennis_Shoes & TapeLift & 24 & 7 \\
\hline Tennis_Shoes & TapeLift & 24 & 8 \\
\hline Tennis_Shoes & TapeLift & 24 & 9 \\
\hline Tennis_Shoes & TapeLift & 24 & 10 \\
\hline Tennis_Shoes & TapeLift & 24 & 11 \\
\hline
\end{tabular}




\begin{tabular}{|c|c|c|c|c|}
\hline Tennis_Shoes & TapeLift & 24 & 12 & 6 \\
\hline Tennis_Shoes & TapeLift & 24 & 13 & 35 \\
\hline Tennis_Shoes & TapeLift & 24 & 14 & 68 \\
\hline Tennis_Shoes & TapeLift & 24 & 15 & 57 \\
\hline Tennis_Shoes & TapeLift & 24 & 16 & 28 \\
\hline Tennis_Shoes & TapeLift & 25 & 1 & 27 \\
\hline Tennis_Shoes & TapeLift & 25 & 2 & 27 \\
\hline Tennis_Shoes & TapeLift & 25 & 3 & 102 \\
\hline Tennis_Shoes & TapeLift & 25 & 4 & 55 \\
\hline Tennis_Shoes & TapeLift & 25 & 5 & 9 \\
\hline Tennis_Shoes & TapeLift & 25 & 6 & 48 \\
\hline Tennis_Shoes & TapeLift & 25 & 7 & 41 \\
\hline Tennis_Shoes & TapeLift & 25 & 8 & 27 \\
\hline Tennis_Shoes & TapeLift & 25 & 9 & 20 \\
\hline Tennis_Shoes & TapeLift & 25 & 10 & 33 \\
\hline Tennis_Shoes & TapeLift & 25 & 11 & 25 \\
\hline Tennis_Shoes & TapeLift & 25 & 12 & 17 \\
\hline Tennis_Shoes & TapeLift & 25 & 13 & 86 \\
\hline Tennis_Shoes & TapeLift & 25 & 14 & 72 \\
\hline Tennis_Shoes & TapeLift & 25 & 15 & 81 \\
\hline Tennis_Shoes & TapeLift & 25 & 16 & 59 \\
\hline Tennis_Shoes & TapeLift & 25 & 17 & 94 \\
\hline Tennis_Shoes & TapeLift & 25 & 18 & 59 \\
\hline Tennis_Shoes & TapeLift & 25 & 19 & 43 \\
\hline Tennis_Shoes & TapeLift & 25 & 20 & 51 \\
\hline Tennis_Shoes & TapeLift & 25 & 21 & 34 \\
\hline Tennis_Shoes & TapeLift & 25 & 22 & 7 \\
\hline Tennis_Shoes & TapeLift & 25 & 23 & 45 \\
\hline Tennis_Shoes & TapeLift & 25 & 24 & 17 \\
\hline Tennis_Shoes & TapeLift & 25 & 25 & 18 \\
\hline Tennis_Shoes & TapeLift & 26 & 1 & 66 \\
\hline Tennis_Shoes & TapeLift & 26 & 2 & 43 \\
\hline Tennis_Shoes & TapeLift & 26 & 3 & 57 \\
\hline Tennis_Shoes & TapeLift & 26 & 4 & 33 \\
\hline Tennis_Shoes & TapeLift & 26 & 5 & 33 \\
\hline Tennis_Shoes & TapeLift & 26 & 6 & 56 \\
\hline Tennis_Shoes & TapeLift & 26 & 7 & 66 \\
\hline Tennis_Shoes & TapeLift & 26 & 8 & 58 \\
\hline Tennis_Shoes & TapeLift & 26 & 9 & 88 \\
\hline Tennis_Shoes & TapeLift & 26 & 10 & 50 \\
\hline Tennis_Shoes & TapeLift & 26 & 11 & 6 \\
\hline Tennis_Shoes & TapeLift & 26 & 12 & 75 \\
\hline Tennis_Shoes & TapeLift & 26 & 13 & 35 \\
\hline Tennis_Shoes & TapeLift & 26 & 14 & 40 \\
\hline Tennis_Shoes & TapeLift & 26 & 15 & 44 \\
\hline
\end{tabular}




\begin{tabular}{|c|c|c|c|c|}
\hline Tennis_Shoes & TapeLift & 26 & 16 & 76 \\
\hline Tennis_Shoes & TapeLift & 26 & 17 & 83 \\
\hline Tennis_Shoes & TapeLift & 26 & 18 & 64 \\
\hline Tennis_Shoes & TapeLift & 26 & 19 & 25 \\
\hline Tennis_Shoes & TapeLift & 26 & 20 & 21 \\
\hline Tennis_Shoes & TapeLift & 27 & 1 & 127 \\
\hline Tennis_Shoes & TapeLift & 27 & 2 & 49 \\
\hline Tennis_Shoes & TapeLift & 27 & 3 & 92 \\
\hline Tennis_Shoes & TapeLift & 27 & 4 & 80 \\
\hline Tennis_Shoes & TapeLift & 27 & 5 & 21 \\
\hline Tennis_Shoes & TapeLift & 27 & 6 & 65 \\
\hline Tennis_Shoes & TapeLift & 27 & 7 & 36 \\
\hline Tennis_Shoes & TapeLift & 27 & 8 & 39 \\
\hline Tennis_Shoes & TapeLift & 27 & 9 & 26 \\
\hline Tennis_Shoes & TapeLift & 27 & 10 & 23 \\
\hline Tennis_Shoes & TapeLift & 27 & 11 & 38 \\
\hline Tennis_Shoes & TapeLift & 27 & 12 & 8 \\
\hline Tennis_Shoes & TapeLift & 27 & 13 & 41 \\
\hline Tennis_Shoes & TapeLift & 27 & 14 & 55 \\
\hline Tennis_Shoes & TapeLift & 27 & 15 & 61 \\
\hline Tennis_Shoes & TapeLift & 27 & 16 & 29 \\
\hline Tennis_Shoes & TapeLift & 27 & 17 & 60 \\
\hline Tennis_Shoes & TapeLift & 28 & 1 & 14 \\
\hline Tennis_Shoes & TapeLift & 28 & 2 & 39 \\
\hline Tennis_Shoes & TapeLift & 28 & 3 & 28 \\
\hline Tennis_Shoes & TapeLift & 28 & 4 & 80 \\
\hline Tennis_Shoes & TapeLift & 28 & 5 & 42 \\
\hline Tennis_Shoes & TapeLift & 28 & 6 & 37 \\
\hline Tennis_Shoes & TapeLift & 28 & 7 & 45 \\
\hline Tennis_Shoes & TapeLift & 28 & 8 & 12 \\
\hline Tennis_Shoes & TapeLift & 28 & 9 & 205 \\
\hline Tennis_Shoes & TapeLift & 28 & 10 & 10 \\
\hline Tennis_Shoes & TapeLift & 28 & 11 & 15 \\
\hline Tennis_Shoes & TapeLift & 28 & 12 & 45 \\
\hline Tennis_Shoes & TapeLift & 28 & 13 & 30 \\
\hline Tennis_Shoes & TapeLift & 28 & 14 & 81 \\
\hline Tennis_Shoes & TapeLift & 28 & 15 & 12 \\
\hline Tennis_Shoes & TapeLift & 28 & 16 & 25 \\
\hline Tennis_Shoes & TapeLift & 28 & 17 & 38 \\
\hline Tennis_Shoes & TapeLift & 28 & 18 & 51 \\
\hline Tennis_Shoes & TapeLift & 28 & 19 & 33 \\
\hline Tennis_Shoes & TapeLift & 28 & 20 & 48 \\
\hline Tennis_Shoes & TapeLift & 28 & 21 & 23 \\
\hline Tennis_Shoes & TapeLift & 28 & 22 & 53 \\
\hline Tennis_Shoes & TapeLift & 29 & 1 & 72 \\
\hline
\end{tabular}




\begin{tabular}{|c|c|c|c|c|}
\hline Tennis_Shoes & TapeLift & 29 & 2 & 85 \\
\hline Tennis_Shoes & TapeLift & 29 & 3 & 80 \\
\hline Tennis_Shoes & TapeLift & 29 & 4 & 46 \\
\hline Tennis_Shoes & TapeLift & 29 & 5 & 40 \\
\hline Tennis_Shoes & TapeLift & 29 & 6 & 59 \\
\hline Tennis_Shoes & TapeLift & 29 & 7 & 80 \\
\hline Tennis_Shoes & TapeLift & 29 & 8 & 46 \\
\hline Tennis_Shoes & TapeLift & 29 & 9 & 32 \\
\hline Tennis_Shoes & TapeLift & 29 & 10 & 20 \\
\hline Tennis_Shoes & TapeLift & 29 & 11 & 43 \\
\hline Tennis_Shoes & TapeLift & 29 & 12 & 47 \\
\hline Tennis_Shoes & TapeLift & 29 & 13 & 51 \\
\hline Tennis_Shoes & TapeLift & 29 & 14 & 30 \\
\hline Tennis_Shoes & TapeLift & 29 & 15 & 52 \\
\hline Tennis_Shoes & TapeLift & 29 & 16 & 28 \\
\hline Tennis_Shoes & TapeLift & 29 & 17 & 17 \\
\hline Tennis_Shoes & TapeLift & 29 & 18 & 52 \\
\hline Tennis_Shoes & TapeLift & 29 & 19 & 50 \\
\hline Tennis_Shoes & TapeLift & 29 & 20 & 47 \\
\hline Tennis_Shoes & TapeLift & 30 & 1 & 62 \\
\hline Tennis_Shoes & TapeLift & 30 & 2 & 42 \\
\hline Tennis_Shoes & TapeLift & 30 & 3 & 65 \\
\hline Tennis_Shoes & TapeLift & 30 & 4 & 13 \\
\hline Tennis_Shoes & TapeLift & 30 & 5 & 41 \\
\hline Tennis_Shoes & TapeLift & 30 & 6 & 35 \\
\hline Tennis_Shoes & TapeLift & 30 & 7 & 24 \\
\hline Tennis_Shoes & TapeLift & 30 & 8 & 10 \\
\hline Tennis_Shoes & TapeLift & 30 & 9 & 16 \\
\hline Tennis_Shoes & TapeLift & 30 & 10 & 45 \\
\hline Tennis_Shoes & TapeLift & 30 & 11 & 46 \\
\hline Tennis_Shoes & TapeLift & 30 & 12 & 16 \\
\hline Tennis_Shoes & TapeLift & 30 & 13 & 34 \\
\hline Tennis_Shoes & TapeLift & 30 & 14 & 27 \\
\hline Tennis_Shoes & TapeLift & 30 & 15 & 38 \\
\hline Tennis_Shoes & TapeLift & 30 & 16 & 40 \\
\hline Tennis_Shoes & TapeLift & 30 & 17 & 45 \\
\hline Tennis_Shoes & TapeLift & 30 & 18 & 39 \\
\hline Tennis_Shoes & TapeLift & 30 & 19 & 59 \\
\hline Tennis_Shoes & TapeLift & 30 & 20 & 38 \\
\hline Tennis_Shoes & TapeLift & 30 & 21 & 33 \\
\hline Tennis_Shoes & TapeLift & 30 & 22 & 27 \\
\hline Tennis_Shoes & TapeLift & 30 & 23 & 36 \\
\hline Tennis_Shoes & TapeLift & 30 & 24 & 40 \\
\hline Tennis_Shoes & TapeLift & 30 & 25 & 15 \\
\hline Tennis_Shoes & TapeLift & 31 & 1 & 12 \\
\hline
\end{tabular}




\begin{tabular}{|c|c|c|c|c|}
\hline Tennis_Shoes & TapeLift & 31 & 2 & 46 \\
\hline Tennis_Shoes & TapeLift & 31 & 3 & 41 \\
\hline Tennis_Shoes & TapeLift & 31 & 4 & 53 \\
\hline Tennis_Shoes & TapeLift & 31 & 5 & 27 \\
\hline Tennis_Shoes & TapeLift & 31 & 6 & 30 \\
\hline Tennis_Shoes & TapeLift & 31 & 7 & 18 \\
\hline Tennis_Shoes & TapeLift & 31 & 8 & 40 \\
\hline Tennis_Shoes & TapeLift & 31 & 9 & 21 \\
\hline Tennis_Shoes & TapeLift & 31 & 10 & 44 \\
\hline Tennis_Shoes & TapeLift & 31 & 11 & 39 \\
\hline Tennis_Shoes & TapeLift & 31 & 12 & 27 \\
\hline Tennis_Shoes & TapeLift & 31 & 13 & 16 \\
\hline Tennis_Shoes & TapeLift & 31 & 14 & 20 \\
\hline Tennis_Shoes & TapeLift & 31 & 15 & 14 \\
\hline Tennis_Shoes & TapeLift & 31 & 16 & 17 \\
\hline Tennis_Shoes & TapeLift & 31 & 17 & 26 \\
\hline Tennis_Shoes & TapeLift & 31 & 18 & 41 \\
\hline Tennis_Shoes & TapeLift & 31 & 19 & 63 \\
\hline Tennis_Shoes & TapeLift & 32 & 1 & 61 \\
\hline Tennis_Shoes & TapeLift & 32 & 2 & 66 \\
\hline Tennis_Shoes & TapeLift & 32 & 3 & 41 \\
\hline Tennis_Shoes & TapeLift & 32 & 4 & 117 \\
\hline Tennis_Shoes & TapeLift & 32 & 5 & 33 \\
\hline Tennis_Shoes & TapeLift & 32 & 6 & 62 \\
\hline Tennis_Shoes & TapeLift & 32 & 7 & 70 \\
\hline Tennis_Shoes & TapeLift & 32 & 8 & 42 \\
\hline Tennis_Shoes & TapeLift & 32 & 9 & 62 \\
\hline Tennis_Shoes & TapeLift & 32 & 10 & 11 \\
\hline Tennis_Shoes & TapeLift & 32 & 11 & 10 \\
\hline Tennis_Shoes & TapeLift & 32 & 12 & 23 \\
\hline Tennis_Shoes & TapeLift & 32 & 13 & 106 \\
\hline Tennis_Shoes & TapeLift & 32 & 14 & 82 \\
\hline Tennis_Shoes & TapeLift & 32 & 15 & 16 \\
\hline Tennis_Shoes & TapeLift & 32 & 16 & 23 \\
\hline Tennis_Shoes & TapeLift & 32 & 17 & 16 \\
\hline Tennis_Shoes & TapeLift & 32 & 18 & 91 \\
\hline Tennis_Shoes & TapeLift & 32 & 19 & 45 \\
\hline Tennis_Shoes & TapeLift & 32 & 20 & 33 \\
\hline Tennis_Shoes & TapeLift & 33 & 1 & 19 \\
\hline Tennis_Shoes & TapeLift & 33 & 2 & 72 \\
\hline Tennis_Shoes & TapeLift & 33 & 3 & 17 \\
\hline Tennis_Shoes & TapeLift & 33 & 4 & 21 \\
\hline Tennis_Shoes & TapeLift & 33 & 5 & 26 \\
\hline Tennis_Shoes & TapeLift & 33 & 6 & 23 \\
\hline Tennis_Shoes & TapeLift & 33 & 7 & 42 \\
\hline
\end{tabular}




\begin{tabular}{|c|c|c|c|c|}
\hline Tennis_Shoes & TapeLift & 33 & 8 & 36 \\
\hline Tennis_Shoes & TapeLift & 33 & 9 & 34 \\
\hline Tennis_Shoes & TapeLift & 33 & 10 & 18 \\
\hline Tennis_Shoes & TapeLift & 33 & 11 & 34 \\
\hline Tennis_Shoes & TapeLift & 33 & 12 & 30 \\
\hline Tennis_Shoes & TapeLift & 33 & 13 & 60 \\
\hline Tennis_Shoes & TapeLift & 33 & 14 & 48 \\
\hline Tennis_Shoes & TapeLift & 33 & 15 & 13 \\
\hline Tennis_Shoes & TapeLift & 33 & 16 & 12 \\
\hline Tennis_Shoes & TapeLift & 33 & 17 & 29 \\
\hline Tennis_Shoes & TapeLift & 33 & 18 & 19 \\
\hline Tennis_Shoes & TapeLift & 33 & 19 & 27 \\
\hline Tennis_Shoes & TapeLift & 33 & 20 & 59 \\
\hline Tennis_Shoes & TapeLift & 34 & 1 & 71 \\
\hline Tennis_Shoes & TapeLift & 34 & 2 & 52 \\
\hline Tennis_Shoes & TapeLift & 34 & 3 & 43 \\
\hline Tennis_Shoes & TapeLift & 34 & 4 & 30 \\
\hline Tennis_Shoes & TapeLift & 34 & 5 & 11 \\
\hline Tennis_Shoes & TapeLift & 34 & 6 & 67 \\
\hline Tennis_Shoes & TapeLift & 34 & 7 & 58 \\
\hline Tennis_Shoes & TapeLift & 34 & 8 & 42 \\
\hline Tennis_Shoes & TapeLift & 34 & 9 & 59 \\
\hline Tennis_Shoes & TapeLift & 34 & 10 & 48 \\
\hline Tennis_Shoes & TapeLift & 34 & 11 & 67 \\
\hline Tennis_Shoes & TapeLift & 34 & 12 & 41 \\
\hline Tennis_Shoes & TapeLift & 34 & 13 & 32 \\
\hline Tennis_Shoes & TapeLift & 34 & 14 & 41 \\
\hline Tennis_Shoes & TapeLift & 34 & 15 & 45 \\
\hline Tennis_Shoes & TapeLift & 34 & 16 & 51 \\
\hline Tennis_Shoes & TapeLift & 34 & 17 & 44 \\
\hline Tennis_Shoes & TapeLift & 34 & 18 & 52 \\
\hline Tennis_Shoes & TapeLift & 35 & 1 & 19 \\
\hline Tennis_Shoes & TapeLift & 35 & 2 & 54 \\
\hline Tennis_Shoes & TapeLift & 35 & 3 & 25 \\
\hline Tennis_Shoes & TapeLift & 35 & 4 & 49 \\
\hline Tennis_Shoes & TapeLift & 35 & 5 & 36 \\
\hline Tennis_Shoes & TapeLift & 35 & 6 & 21 \\
\hline Tennis_Shoes & TapeLift & 35 & 7 & 91 \\
\hline Tennis_Shoes & TapeLift & 35 & 8 & 64 \\
\hline Tennis_Shoes & TapeLift & 35 & 9 & 85 \\
\hline Tennis_Shoes & TapeLift & 35 & 10 & 56 \\
\hline Tennis_Shoes & TapeLift & 35 & 11 & 30 \\
\hline Tennis_Shoes & TapeLift & 35 & 12 & 54 \\
\hline Tennis_Shoes & TapeLift & 35 & 13 & 40 \\
\hline Tennis_Shoes & TapeLift & 35 & 14 & 44 \\
\hline
\end{tabular}




\begin{tabular}{|c|c|}
\hline Tennis_Shoes & TapeLift \\
\hline Tennis_Shoes & \\
\hline Tennis_Shoes & \\
\hline Tennis_Shoes & \\
\hline Tennis_Shoes & \\
\hline Tennis_Shoes & \\
\hline Tennis_Shoes & TapeL \\
\hline Tennis_Shoes & \\
\hline Tennis_Shoes & \\
\hline Tennis_Shoes & Tape \\
\hline Tennis_Shoes & \\
\hline Tennis_Shoes & \\
\hline Tennis_Shoes & \\
\hline Tennis_Shoes & \\
\hline Tennis_Shoes & \\
\hline Tennis_Shoes & Vacu \\
\hline Tennis_Shoes & \\
\hline Tennis_Shoes & \\
\hline Tennis_Shoes & \\
\hline Tennis_Shoes & \\
\hline Tennis_Shoes & \\
\hline Tennis_Shoes & Vacu \\
\hline Tennis_Shoes & Vacu \\
\hline Tennis_Shoes & \\
\hline Tennis_Shoes & Vacu \\
\hline Tennis_Shoes & Vacu \\
\hline Tennis_Shoes & Vacu \\
\hline Tennis_Shoes & Vacu \\
\hline Tennis_Shoes & Vacu \\
\hline Tennis_Shoes & Vacu \\
\hline Tennis_Shoes & Vacu \\
\hline Tennis_Shoes & Vacu \\
\hline Tennis_Shoes & Vacu \\
\hline Tennis_Shoes & Vacu \\
\hline Tennis_Shoes & Vacu \\
\hline Tennis_Shoes & Vacu \\
\hline Tennis_Shoes & Vacu \\
\hline Tennis_Shoes & Vacu \\
\hline Tennis_Shoes & Vacul \\
\hline Tennis_Shoes & Vacu \\
\hline Tennis_Shoes & Vacu \\
\hline Tennis_Shoes & Vacu \\
\hline Tennis_Shoes & Vacul \\
\hline Tennis_Shoes & Vacul \\
\hline Tennis_Shoes & Vacu \\
\hline
\end{tabular}

\begin{tabular}{|c|c|}
\hline 35 & 15 \\
\hline 35 & 16 \\
\hline 35 & 17 \\
\hline 35 & 18 \\
\hline 35 & 19 \\
\hline 35 & 20 \\
\hline 35 & 21 \\
\hline 35 & 22 \\
\hline 35 & 23 \\
\hline 35 & 24 \\
\hline 35 & 25 \\
\hline 1 & 1 \\
\hline 1 & 2 \\
\hline 1 & 3 \\
\hline 1 & 4 \\
\hline 1 & 5 \\
\hline 1 & 6 \\
\hline 1 & 7 \\
\hline 1 & 8 \\
\hline 1 & 9 \\
\hline 1 & 10 \\
\hline 1 & 11 \\
\hline 2 & 1 \\
\hline 2 & 2 \\
\hline 2 & 3 \\
\hline 2 & 4 \\
\hline 2 & 5 \\
\hline 2 & 6 \\
\hline 2 & 7 \\
\hline 2 & 8 \\
\hline 2 & 9 \\
\hline 2 & 10 \\
\hline 2 & 11 \\
\hline 3 & 1 \\
\hline 3 & 2 \\
\hline 3 & 3 \\
\hline 3 & 4 \\
\hline 3 & 5 \\
\hline 3 & 6 \\
\hline 3 & 7 \\
\hline 3 & 8 \\
\hline 3 & 9 \\
\hline 3 & 10 \\
\hline 4 & 1 \\
\hline 4 & 2 \\
\hline
\end{tabular}




\begin{tabular}{|c|c|c|c|c|}
\hline Tennis_Shoes & Vacuum & 4 & 3 & 30 \\
\hline Tennis_Shoes & Vacuum & 4 & 4 & 53 \\
\hline Tennis_Shoes & Vacuum & 4 & 5 & 49 \\
\hline Tennis_Shoes & Vacuum & 4 & 6 & 30 \\
\hline Tennis_Shoes & Vacuum & 4 & 7 & 27 \\
\hline Tennis_Shoes & Vacuum & 4 & 8 & 19 \\
\hline Tennis_Shoes & Vacuum & 4 & 9 & 23 \\
\hline Tennis_Shoes & Vacuum & 4 & 10 & 18 \\
\hline Tennis_Shoes & Vacuum & 4 & 11 & 30 \\
\hline Tennis_Shoes & Vacuum & 5 & 1 & 15 \\
\hline Tennis_Shoes & Vacuum & 5 & 2 & 9 \\
\hline Tennis_Shoes & Vacuum & 5 & 3 & 10 \\
\hline Tennis_Shoes & Vacuum & 5 & 4 & 6 \\
\hline Tennis_Shoes & Vacuum & 5 & 5 & 48 \\
\hline Tennis_Shoes & Vacuum & 5 & 6 & 13 \\
\hline Tennis_Shoes & Vacuum & 6 & 1 & 7 \\
\hline Tennis_Shoes & Vacuum & 6 & 2 & 11 \\
\hline Tennis_Shoes & Vacuum & 6 & 3 & 28 \\
\hline Tennis_Shoes & Vacuum & 6 & 4 & 17 \\
\hline Tennis_Shoes & Vacuum & 6 & 5 & 15 \\
\hline Tennis_Shoes & Vacuum & 6 & 6 & 8 \\
\hline Tennis_Shoes & Vacuum & 6 & 7 & 27 \\
\hline Tennis_Shoes & Vacuum & 6 & 8 & 33 \\
\hline Tennis_Shoes & Vacuum & 6 & 9 & 15 \\
\hline Tennis_Shoes & Vacuum & 6 & 10 & 15 \\
\hline Tennis_Shoes & Vacuum & 6 & 11 & 4 \\
\hline Tennis_Shoes & Vacuum & 7 & 1 & 43 \\
\hline Tennis_Shoes & Vacuum & 7 & 2 & 19 \\
\hline Tennis_Shoes & Vacuum & 7 & 3 & 37 \\
\hline Tennis_Shoes & Vacuum & 7 & 4 & 14 \\
\hline Tennis_Shoes & Vacuum & 7 & 5 & 24 \\
\hline Tennis_Shoes & Vacuum & 8 & 1 & 1 \\
\hline Tennis_Shoes & Vacuum & 8 & 2 & 33 \\
\hline Tennis_Shoes & Vacuum & 8 & 3 & 22 \\
\hline Tennis_Shoes & Vacuum & 8 & 4 & 26 \\
\hline Tennis_Shoes & Vacuum & 8 & 5 & 22 \\
\hline Tennis_Shoes & Vacuum & 8 & 6 & 16 \\
\hline Tennis_Shoes & Vacuum & 8 & 7 & 32 \\
\hline Tennis_Shoes & Vacuum & 8 & 8 & 26 \\
\hline Tennis_Shoes & Vacuum & 8 & 9 & 26 \\
\hline Tennis_Shoes & Vacuum & 8 & 10 & 21 \\
\hline Tennis_Shoes & Vacuum & 8 & 11 & 29 \\
\hline Tennis_Shoes & Vacuum & 8 & 12 & 9 \\
\hline Tennis_Shoes & Vacuum & 8 & 13 & 43 \\
\hline Tennis_Shoes & Vacuum & 8 & 14 & 30 \\
\hline
\end{tabular}




\begin{tabular}{|c|c|c|c|c|}
\hline Tennis_Shoes & Vacuum & 8 & 15 & 27 \\
\hline Tennis_Shoes & Vacuum & 8 & 16 & 24 \\
\hline Tennis_Shoes & Vacuum & 8 & 17 & 8 \\
\hline Tennis_Shoes & Vacuum & 8 & 18 & 13 \\
\hline Tennis_Shoes & Vacuum & 9 & 1 & 47 \\
\hline Tennis_Shoes & Vacuum & 9 & 2 & 40 \\
\hline Tennis_Shoes & Vacuum & 9 & 3 & 25 \\
\hline Tennis_Shoes & Vacuum & 9 & 4 & 14 \\
\hline Tennis_Shoes & Vacuum & 9 & 5 & 15 \\
\hline Tennis_Shoes & Vacuum & 9 & 6 & 10 \\
\hline Tennis_Shoes & Vacuum & 9 & 7 & 32 \\
\hline Tennis_Shoes & Vacuum & 9 & 8 & 32 \\
\hline Tennis_Shoes & Vacuum & 9 & 9 & 20 \\
\hline Tennis_Shoes & Vacuum & 9 & 10 & 28 \\
\hline Tennis_Shoes & Vacuum & 9 & 11 & 3 \\
\hline Tennis_Shoes & Vacuum & 9 & 12 & 3 \\
\hline Tennis_Shoes & Vacuum & 9 & 13 & 7 \\
\hline Tennis_Shoes & Vacuum & 9 & 14 & 6 \\
\hline Tennis_Shoes & Vacuum & 9 & 15 & 3 \\
\hline Tennis_Shoes & Vacuum & 9 & 16 & 5 \\
\hline Tennis_Shoes & Vacuum & 9 & 17 & 2 \\
\hline Tennis_Shoes & Vacuum & 9 & 18 & 6 \\
\hline Tennis_Shoes & Vacuum & 9 & 19 & 7 \\
\hline Tennis_Shoes & Vacuum & 9 & 20 & 28 \\
\hline Tennis_Shoes & Vacuum & 9 & 21 & 10 \\
\hline Tennis_Shoes & Vacuum & 10 & 1 & 15 \\
\hline Tennis_Shoes & Vacuum & 10 & 2 & 12 \\
\hline Tennis_Shoes & Vacuum & 10 & 3 & 19 \\
\hline Tennis_Shoes & Vacuum & 10 & 4 & 33 \\
\hline Tennis_Shoes & Vacuum & 10 & 5 & 21 \\
\hline Tennis_Shoes & Vacuum & 10 & 6 & 27 \\
\hline Tennis_Shoes & Vacuum & 10 & 7 & 4 \\
\hline Tennis_Shoes & Vacuum & 10 & 8 & 6 \\
\hline Tennis_Shoes & Vacuum & 10 & 9 & 12 \\
\hline Tennis_Shoes & Vacuum & 10 & 10 & 25 \\
\hline Tennis_Shoes & Vacuum & 10 & 11 & 4 \\
\hline Tennis_Shoes & Vacuum & 10 & 12 & 12 \\
\hline Tennis_Shoes & Vacuum & 10 & 13 & 12 \\
\hline Tennis_Shoes & Vacuum & 10 & 14 & 2 \\
\hline Tennis_Shoes & Vacuum & 10 & 15 & 17 \\
\hline Tennis_Shoes & Vacuum & 10 & 16 & 6 \\
\hline Tennis_Shoes & Vacuum & 11 & 1 & 47 \\
\hline Tennis_Shoes & Vacuum & 11 & 2 & 5 \\
\hline Tennis_Shoes & Vacuum & 11 & 3 & 4 \\
\hline Tennis_Shoes & Vacuum & 11 & 4 & 2 \\
\hline
\end{tabular}




\begin{tabular}{|c|c|}
\hline Tennis_Shoes & Vacuum \\
\hline Tennis_Shoes & Vacuum \\
\hline Tennis_Shoes & Vacuum \\
\hline Tennis_Shoes & Vacuum \\
\hline Tennis_Shoes & Vacuum \\
\hline Tennis_Shoes & Vacuum \\
\hline Tennis_Shoes & Vacuum \\
\hline Tennis_Shoes & Vacuum \\
\hline Tennis_Shoes & Vacuum \\
\hline Tennis_Shoes & Vacuum \\
\hline Tennis_Shoes & Vacuum \\
\hline Tennis_Shoes & Vacuum \\
\hline Tennis_Shoes & Vacuum \\
\hline Tennis_Shoes & Vacuum \\
\hline Tennis_Shoes & Vacuum \\
\hline Tennis_Shoes & Vacuum \\
\hline Tennis_Shoes & Vacuum \\
\hline Tennis_Shoes & Vacuum \\
\hline Tennis_Shoes & Vacuum \\
\hline Tennis_Shoes & Vacuum \\
\hline Tennis_Shoes & Vacuum \\
\hline Tennis_Shoes & Vacuum \\
\hline Tennis_Shoes & Vacuum \\
\hline Tennis_Shoes & Vacuum \\
\hline Tennis_Shoes & Vacuum \\
\hline Tennis_Shoes & Vacuum \\
\hline Tennis_Shoes & Vacuum \\
\hline Tennis_Shoes & Vacuum \\
\hline Tennis_Shoes & Vacuum \\
\hline Tennis_Shoes & Vacuum \\
\hline Tennis_Shoes & Vacuum \\
\hline Tennis_Shoes & Vacuum \\
\hline Tennis_Shoes & Vacuum \\
\hline Tennis_Shoes & Vacuum \\
\hline Tennis_Shoes & Vacuum \\
\hline Tennis_Shoes & Vacuum \\
\hline Tennis_Shoes & Vacuum \\
\hline Tennis_Shoes & Vacuum \\
\hline Tennis_Shoes & Vacuum \\
\hline Tennis_Shoes & Vacuum \\
\hline Tennis_Shoes & Vacuum \\
\hline Tennis_Shoes & Vacuum \\
\hline Tennis_Shoes & Vacuum \\
\hline Tennis_Shoes & Vacuum \\
\hline Tennis_Shoes & Vacuum \\
\hline
\end{tabular}

\begin{tabular}{|c|c|}
\hline 11 & 5 \\
\hline 11 & 6 \\
\hline 11 & 7 \\
\hline 11 & 8 \\
\hline 11 & 9 \\
\hline 11 & 10 \\
\hline 11 & 11 \\
\hline 11 & 12 \\
\hline 11 & 13 \\
\hline 11 & 14 \\
\hline 11 & 15 \\
\hline 11 & 16 \\
\hline 11 & 17 \\
\hline 11 & 18 \\
\hline 11 & 19 \\
\hline 11 & 20 \\
\hline 12 & 1 \\
\hline 12 & 2 \\
\hline 12 & 3 \\
\hline 12 & 4 \\
\hline 12 & 5 \\
\hline 12 & 6 \\
\hline 12 & 7 \\
\hline 12 & 8 \\
\hline 12 & 9 \\
\hline 12 & 10 \\
\hline 12 & 11 \\
\hline 12 & 12 \\
\hline 13 & 1 \\
\hline 13 & 2 \\
\hline 13 & 3 \\
\hline 13 & 4 \\
\hline 13 & 5 \\
\hline 13 & 6 \\
\hline 13 & 7 \\
\hline 13 & 8 \\
\hline 13 & 9 \\
\hline 13 & 10 \\
\hline 13 & 11 \\
\hline 13 & 12 \\
\hline 14 & 1 \\
\hline 14 & 2 \\
\hline 14 & 3 \\
\hline 14 & 4 \\
\hline 14 & 5 \\
\hline
\end{tabular}




\begin{tabular}{|c|c|}
\hline Tennis_Shoes & Vacuum \\
\hline Tennis_Shoes & Vacuum \\
\hline Tennis_Shoes & Vacuum \\
\hline Tennis_Shoes & Vacuum \\
\hline Tennis_Shoes & Vacuum \\
\hline Tennis_Shoes & Vacuum \\
\hline Tennis_Shoes & Vacuum \\
\hline Tennis_Shoes & Vacuum \\
\hline Tennis_Shoes & Vacuum \\
\hline Tennis_Shoes & Vacuum \\
\hline Tennis_Shoes & Vacuum \\
\hline Tennis_Shoes & Vacuum \\
\hline Tennis_Shoes & Vacuum \\
\hline Tennis_Shoes & Vacuum \\
\hline Tennis_Shoes & Vacuum \\
\hline Tennis_Shoes & Vacuum \\
\hline Tennis_Shoes & Vacuum \\
\hline Tennis_Shoes & Vacuum \\
\hline Tennis_Shoes & Vacuum \\
\hline Tennis_Shoes & Vacuum \\
\hline Tennis_Shoes & Vacuum \\
\hline Tennis_Shoes & Vacuum \\
\hline Tennis_Shoes & Vacuum \\
\hline Tennis_Shoes & Vacuum \\
\hline Tennis_Shoes & Vacuum \\
\hline Tennis_Shoes & Vacuum \\
\hline Tennis_Shoes & Vacuum \\
\hline Tennis_Shoes & Vacuum \\
\hline Tennis_Shoes & Vacuum \\
\hline Tennis_Shoes & Vacuum \\
\hline Tennis_Shoes & Vacuum \\
\hline Tennis_Shoes & Vacuum \\
\hline Tennis_Shoes & Vacuum \\
\hline Tennis_Shoes & Vacuum \\
\hline Tennis_Shoes & Vacuum \\
\hline Tennis_Shoes & Vacuum \\
\hline Tennis_Shoes & Vacuum \\
\hline Tennis_Shoes & Vacuum \\
\hline Tennis_Shoes & Vacuum \\
\hline Tennis_Shoes & Vacuum \\
\hline Tennis_Shoes & Vacuum \\
\hline Tennis_Shoes & Vacuum \\
\hline Tennis_Shoes & Vacuum \\
\hline Tennis_Shoes & Vacuum \\
\hline Tennis_Shoes & Vacuum \\
\hline
\end{tabular}

\begin{tabular}{|c|c|}
\hline 14 & 6 \\
\hline 14 & 7 \\
\hline 14 & 8 \\
\hline 14 & 9 \\
\hline 14 & 10 \\
\hline 14 & 11 \\
\hline 14 & 12 \\
\hline 14 & 13 \\
\hline 14 & 14 \\
\hline 14 & 15 \\
\hline 15 & 1 \\
\hline 15 & 2 \\
\hline 15 & 3 \\
\hline 15 & 4 \\
\hline 15 & 5 \\
\hline 15 & 6 \\
\hline 15 & 7 \\
\hline 15 & 8 \\
\hline 15 & 9 \\
\hline 15 & 10 \\
\hline 15 & 11 \\
\hline 15 & 12 \\
\hline 15 & 13 \\
\hline 15 & 14 \\
\hline 15 & 15 \\
\hline 15 & 16 \\
\hline 16 & 1 \\
\hline 16 & 2 \\
\hline 16 & 3 \\
\hline 16 & 4 \\
\hline 16 & 5 \\
\hline 16 & 6 \\
\hline 16 & 7 \\
\hline 16 & 8 \\
\hline 16 & 9 \\
\hline 16 & 10 \\
\hline 16 & 11 \\
\hline 16 & 12 \\
\hline 17 & 1 \\
\hline 17 & 2 \\
\hline 17 & 3 \\
\hline 17 & 4 \\
\hline 17 & 5 \\
\hline 17 & 6 \\
\hline 17 & 7 \\
\hline
\end{tabular}




\begin{tabular}{|c|c|c|c|c|}
\hline Tennis_Shoes & Vacuum & 17 & 8 & 35 \\
\hline Tennis_Shoes & Vacuum & 17 & 9 & 16 \\
\hline Tennis_Shoes & Vacuum & 17 & 10 & 22 \\
\hline Tennis_Shoes & Vacuum & 17 & 11 & 11 \\
\hline Tennis_Shoes & Vacuum & 17 & 12 & 3 \\
\hline Tennis_Shoes & Vacuum & 17 & 13 & 15 \\
\hline Tennis_Shoes & Vacuum & 17 & 14 & 26 \\
\hline Tennis_Shoes & Vacuum & 17 & 15 & 17 \\
\hline Tennis_Shoes & Vacuum & 17 & 16 & 17 \\
\hline Tennis_Shoes & Vacuum & 17 & 17 & 36 \\
\hline Tennis_Shoes & Vacuum & 18 & 1 & 14 \\
\hline Tennis_Shoes & Vacuum & 18 & 2 & 20 \\
\hline Tennis_Shoes & Vacuum & 18 & 3 & 14 \\
\hline Tennis_Shoes & Vacuum & 18 & 4 & 45 \\
\hline Tennis_Shoes & Vacuum & 18 & 5 & 39 \\
\hline Tennis_Shoes & Vacuum & 18 & 6 & 20 \\
\hline Tennis_Shoes & Vacuum & 18 & 7 & 39 \\
\hline Tennis_Shoes & Vacuum & 18 & 8 & 8 \\
\hline Tennis_Shoes & Vacuum & 18 & 9 & 20 \\
\hline Tennis_Shoes & Vacuum & 18 & 10 & 2 \\
\hline Tennis_Shoes & Vacuum & 18 & 11 & 26 \\
\hline Tennis_Shoes & Vacuum & 18 & 12 & 19 \\
\hline Tennis_Shoes & Vacuum & 18 & 13 & 16 \\
\hline Tennis_Shoes & Vacuum & 18 & 14 & 28 \\
\hline Tennis_Shoes & Vacuum & 18 & 15 & 34 \\
\hline Tennis_Shoes & Vacuum & 18 & 16 & 34 \\
\hline Tennis_Shoes & Vacuum & 18 & 17 & 37 \\
\hline Tennis_Shoes & Vacuum & 18 & 18 & 30 \\
\hline Tennis_Shoes & Vacuum & 18 & 19 & 23 \\
\hline Tennis_Shoes & Vacuum & 18 & 20 & 31 \\
\hline Tennis_Shoes & Vacuum & 18 & 21 & 20 \\
\hline Tennis_Shoes & Vacuum & 18 & 22 & 36 \\
\hline Tennis_Shoes & Vacuum & 19 & 1 & 20 \\
\hline Tennis_Shoes & Vacuum & 19 & 2 & 13 \\
\hline Tennis_Shoes & Vacuum & 19 & 3 & 7 \\
\hline Tennis_Shoes & Vacuum & 19 & 4 & 16 \\
\hline Tennis_Shoes & Vacuum & 19 & 5 & 14 \\
\hline Tennis_Shoes & Vacuum & 19 & 6 & 6 \\
\hline Tennis_Shoes & Vacuum & 19 & 7 & 13 \\
\hline Tennis_Shoes & Vacuum & 19 & 8 & 25 \\
\hline Tennis_Shoes & Vacuum & 19 & 9 & 20 \\
\hline Tennis_Shoes & Vacuum & 19 & 10 & 24 \\
\hline Tennis_Shoes & Vacuum & 19 & 11 & 21 \\
\hline Tennis_Shoes & Vacuum & 19 & 12 & 29 \\
\hline Tennis_Shoes & Vacuum & 19 & 13 & 24 \\
\hline
\end{tabular}




\begin{tabular}{|c|c|c|c|c|}
\hline Tennis_Shoes & Vacuum & 19 & 14 & 16 \\
\hline Tennis_Shoes & Vacuum & 19 & 15 & 27 \\
\hline Tennis_Shoes & Vacuum & 19 & 16 & 15 \\
\hline Tennis_Shoes & Vacuum & 20 & 1 & 25 \\
\hline Tennis_Shoes & Vacuum & 20 & 2 & 10 \\
\hline Tennis_Shoes & Vacuum & 20 & 3 & 13 \\
\hline Tennis_Shoes & Vacuum & 20 & 4 & 15 \\
\hline Tennis_Shoes & Vacuum & 20 & 5 & 31 \\
\hline Tennis_Shoes & Vacuum & 20 & 6 & 10 \\
\hline Tennis_Shoes & Vacuum & 20 & 7 & 18 \\
\hline Tennis_Shoes & Vacuum & 20 & 8 & 23 \\
\hline Tennis_Shoes & Vacuum & 20 & 9 & 15 \\
\hline Tennis_Shoes & Vacuum & 20 & 10 & 18 \\
\hline Tennis_Shoes & Vacuum & 21 & 1 & 4 \\
\hline Tennis_Shoes & Vacuum & 21 & 2 & 25 \\
\hline Tennis_Shoes & Vacuum & 21 & 3 & 6 \\
\hline Tennis_Shoes & Vacuum & 21 & 4 & 15 \\
\hline Tennis_Shoes & Vacuum & 21 & 5 & 11 \\
\hline Tennis_Shoes & Vacuum & 21 & 6 & 25 \\
\hline Tennis_Shoes & Vacuum & 21 & 7 & 17 \\
\hline Tennis_Shoes & Vacuum & 21 & 8 & 3 \\
\hline Tennis_Shoes & Vacuum & 21 & 9 & 4 \\
\hline Tennis_Shoes & Vacuum & 21 & 10 & 10 \\
\hline Tennis_Shoes & Vacuum & 21 & 11 & 91 \\
\hline Tennis_Shoes & Vacuum & 21 & 12 & 11 \\
\hline Tennis_Shoes & Vacuum & 21 & 13 & 7 \\
\hline Tennis_Shoes & Vacuum & 21 & 14 & 22 \\
\hline Tennis_Shoes & Vacuum & 22 & 1 & 27 \\
\hline Tennis_Shoes & Vacuum & 22 & 2 & 27 \\
\hline Tennis_Shoes & Vacuum & 22 & 3 & 23 \\
\hline Tennis_Shoes & Vacuum & 22 & 4 & 44 \\
\hline Tennis_Shoes & Vacuum & 22 & 5 & 29 \\
\hline Tennis_Shoes & Vacuum & 22 & 6 & 26 \\
\hline Tennis_Shoes & Vacuum & 22 & 7 & 29 \\
\hline Tennis_Shoes & Vacuum & 22 & 8 & 43 \\
\hline Tennis_Shoes & Vacuum & 22 & 9 & 33 \\
\hline Tennis_Shoes & Vacuum & 22 & 10 & 38 \\
\hline Tennis_Shoes & Vacuum & 22 & 11 & 28 \\
\hline Tennis_Shoes & Vacuum & 23 & 1 & 25 \\
\hline Tennis_Shoes & Vacuum & 23 & 2 & 21 \\
\hline Tennis_Shoes & Vacuum & 23 & 3 & 33 \\
\hline Tennis_Shoes & Vacuum & 23 & 4 & 26 \\
\hline Tennis_Shoes & Vacuum & 23 & 5 & 24 \\
\hline Tennis_Shoes & Vacuum & 23 & 6 & 27 \\
\hline Tennis_Shoes & Vacuum & 23 & 7 & 22 \\
\hline
\end{tabular}




\begin{tabular}{|c|c|c|c|c|}
\hline Tennis_Shoes & Vacuum & 23 & 8 & 25 \\
\hline Tennis_Shoes & Vacuum & 23 & 9 & 24 \\
\hline Tennis_Shoes & Vacuum & 23 & 10 & 28 \\
\hline Tennis_Shoes & Vacuum & 23 & 11 & 11 \\
\hline Tennis_Shoes & Vacuum & 23 & 12 & 20 \\
\hline Tennis_Shoes & Vacuum & 23 & 13 & 23 \\
\hline Tennis_Shoes & Vacuum & 23 & 14 & 3 \\
\hline Tennis_Shoes & Vacuum & 23 & 15 & 1 \\
\hline Tennis_Shoes & Vacuum & 23 & 16 & 3 \\
\hline Tennis_Shoes & Vacuum & 24 & 1 & 31 \\
\hline Tennis_Shoes & Vacuum & 24 & 2 & 10 \\
\hline Tennis_Shoes & Vacuum & 24 & 3 & 13 \\
\hline Tennis_Shoes & Vacuum & 24 & 4 & 18 \\
\hline Tennis_Shoes & Vacuum & 24 & 5 & 19 \\
\hline Tennis_Shoes & Vacuum & 24 & 6 & 32 \\
\hline Tennis_Shoes & Vacuum & 25 & 1 & 17 \\
\hline Tennis_Shoes & Vacuum & 25 & 2 & 21 \\
\hline Tennis_Shoes & Vacuum & 25 & 3 & 32 \\
\hline Tennis_Shoes & Vacuum & 25 & 4 & 22 \\
\hline Tennis_Shoes & Vacuum & 25 & 5 & 21 \\
\hline Tennis_Shoes & Vacuum & 25 & 6 & 25 \\
\hline Tennis_Shoes & Vacuum & 25 & 7 & 30 \\
\hline Tennis_Shoes & Vacuum & 25 & 8 & 20 \\
\hline Tennis_Shoes & Vacuum & 26 & 1 & 28 \\
\hline Tennis_Shoes & Vacuum & 26 & 2 & 22 \\
\hline Tennis_Shoes & Vacuum & 26 & 3 & 40 \\
\hline Tennis_Shoes & Vacuum & 26 & 4 & 12 \\
\hline Tennis_Shoes & Vacuum & 26 & 5 & 24 \\
\hline Tennis_Shoes & Vacuum & 26 & 6 & 45 \\
\hline Tennis_Shoes & Vacuum & 26 & 7 & 31 \\
\hline Tennis_Shoes & Vacuum & 26 & 8 & 26 \\
\hline Tennis_Shoes & Vacuum & 26 & 9 & 25 \\
\hline Tennis_Shoes & Vacuum & 26 & 10 & 22 \\
\hline Tennis_Shoes & Vacuum & 26 & 11 & 30 \\
\hline Tennis_Shoes & Vacuum & 26 & 12 & 7 \\
\hline Tennis_Shoes & Vacuum & 26 & 13 & 32 \\
\hline Tennis_Shoes & Vacuum & 26 & 14 & 35 \\
\hline Tennis_Shoes & Vacuum & 26 & 15 & 29 \\
\hline Tennis_Shoes & Vacuum & 26 & 16 & 15 \\
\hline Tennis_Shoes & Vacuum & 27 & 1 & 43 \\
\hline Tennis_Shoes & Vacuum & 27 & 2 & 20 \\
\hline Tennis_Shoes & Vacuum & 27 & 3 & 3 \\
\hline Tennis_Shoes & Vacuum & 27 & 4 & 36 \\
\hline Tennis_Shoes & Vacuum & 27 & 5 & 29 \\
\hline Tennis_Shoes & Vacuum & 27 & 6 & 32 \\
\hline
\end{tabular}




\begin{tabular}{|c|c|c|c|c|}
\hline Tennis_Shoes & Vacuum & 27 & 7 & 18 \\
\hline Tennis_Shoes & Vacuum & 27 & 8 & 11 \\
\hline Tennis_Shoes & Vacuum & 27 & 9 & 12 \\
\hline Tennis_Shoes & Vacuum & 27 & 10 & 12 \\
\hline Tennis_Shoes & Vacuum & 28 & 1 & 28 \\
\hline Tennis_Shoes & Vacuum & 28 & 2 & 3 \\
\hline Tennis_Shoes & Vacuum & 28 & 3 & 20 \\
\hline Tennis_Shoes & Vacuum & 28 & 4 & 28 \\
\hline Tennis_Shoes & Vacuum & 28 & 5 & 20 \\
\hline Tennis_Shoes & Vacuum & 28 & 6 & 10 \\
\hline Tennis_Shoes & Vacuum & 28 & 7 & 8 \\
\hline Tennis_Shoes & Vacuum & 28 & 8 & 9 \\
\hline Tennis_Shoes & Vacuum & 28 & 9 & 5 \\
\hline Tennis_Shoes & Vacuum & 28 & 10 & 40 \\
\hline Tennis_Shoes & Vacuum & 28 & 11 & 32 \\
\hline Tennis_Shoes & Vacuum & 28 & 12 & 21 \\
\hline Tennis_Shoes & Vacuum & 28 & 13 & 19 \\
\hline Tennis_Shoes & Vacuum & 28 & 14 & 34 \\
\hline Tennis_Shoes & Vacuum & 28 & 15 & 21 \\
\hline Tennis_Shoes & Vacuum & 29 & 1 & 39 \\
\hline Tennis_Shoes & Vacuum & 29 & 2 & 24 \\
\hline Tennis_Shoes & Vacuum & 29 & 3 & 16 \\
\hline Tennis_Shoes & Vacuum & 29 & 4 & 12 \\
\hline Tennis_Shoes & Vacuum & 29 & 5 & 7 \\
\hline Tennis_Shoes & Vacuum & 29 & 6 & 12 \\
\hline Tennis_Shoes & Vacuum & 29 & 7 & 27 \\
\hline Tennis_Shoes & Vacuum & 29 & 8 & 8 \\
\hline Tennis_Shoes & Vacuum & 29 & 9 & 30 \\
\hline Tennis_Shoes & Vacuum & 29 & 10 & 43 \\
\hline Tennis_Shoes & Vacuum & 29 & 11 & 20 \\
\hline Tennis_Shoes & Vacuum & 29 & 12 & 12 \\
\hline Tennis_Shoes & Vacuum & 29 & 13 & 5 \\
\hline Tennis_Shoes & Vacuum & 29 & 14 & 10 \\
\hline Tennis_Shoes & Vacuum & 29 & 15 & 21 \\
\hline Tennis_Shoes & Vacuum & 29 & 16 & 30 \\
\hline Tennis_Shoes & Vacuum & 29 & 17 & 23 \\
\hline Tennis_Shoes & Vacuum & 29 & 18 & 25 \\
\hline Tennis_Shoes & Vacuum & 29 & 19 & 10 \\
\hline Tennis_Shoes & Vacuum & 29 & 20 & 30 \\
\hline Tennis_Shoes & Vacuum & 30 & 1 & 24 \\
\hline Tennis_Shoes & Vacuum & 30 & 2 & 21 \\
\hline Tennis_Shoes & Vacuum & 30 & 3 & 21 \\
\hline Tennis_Shoes & Vacuum & 30 & 4 & 24 \\
\hline Tennis_Shoes & Vacuum & 30 & 5 & 29 \\
\hline Tennis_Shoes & Vacuum & 30 & 6 & 5 \\
\hline
\end{tabular}




\begin{tabular}{|c|c|c|c|c|}
\hline Tennis_Shoes & Vacuum & 30 & 7 & 26 \\
\hline Tennis_Shoes & Vacuum & 30 & 8 & 22 \\
\hline Tennis_Shoes & Vacuum & 30 & 9 & 4 \\
\hline Tennis_Shoes & Vacuum & 30 & 10 & 30 \\
\hline Tennis_Shoes & Vacuum & 30 & 11 & 18 \\
\hline Tennis_Shoes & Vacuum & 30 & 12 & 12 \\
\hline Tennis_Shoes & Vacuum & 30 & 13 & 1 \\
\hline Tennis_Shoes & Vacuum & 30 & 14 & 4 \\
\hline Tennis_Shoes & Vacuum & 30 & 15 & 12 \\
\hline Tennis_Shoes & Vacuum & 30 & 16 & 11 \\
\hline Tennis_Shoes & Vacuum & 30 & 17 & 4 \\
\hline Tennis_Shoes & Vacuum & 30 & 18 & 28 \\
\hline Tennis_Shoes & Vacuum & 30 & 19 & 1 \\
\hline Tennis_Shoes & Vacuum & 30 & 20 & 8 \\
\hline Tennis_Shoes & Vacuum & 30 & 21 & 25 \\
\hline Tennis_Shoes & Vacuum & 30 & 22 & 28 \\
\hline Tennis_Shoes & Vacuum & 30 & 23 & 2 \\
\hline Tennis_Shoes & Vacuum & 31 & 1 & 23 \\
\hline Tennis_Shoes & Vacuum & 31 & 2 & 7 \\
\hline Tennis_Shoes & Vacuum & 31 & 3 & 23 \\
\hline Tennis_Shoes & Vacuum & 31 & 4 & 37 \\
\hline Tennis_Shoes & Vacuum & 31 & 5 & 17 \\
\hline Tennis_Shoes & Vacuum & 31 & 6 & 40 \\
\hline Tennis_Shoes & Vacuum & 31 & 7 & 37 \\
\hline Tennis_Shoes & Vacuum & 31 & 8 & 46 \\
\hline Tennis_Shoes & Vacuum & 31 & 9 & 10 \\
\hline Tennis_Shoes & Vacuum & 31 & 10 & 9 \\
\hline Tennis_Shoes & Vacuum & 31 & 11 & 26 \\
\hline Tennis_Shoes & Vacuum & 31 & 12 & 10 \\
\hline Tennis_Shoes & Vacuum & 32 & 1 & 6 \\
\hline Tennis_Shoes & Vacuum & 32 & 2 & 5 \\
\hline Tennis_Shoes & Vacuum & 32 & 3 & 90 \\
\hline Tennis_Shoes & Vacuum & 32 & 4 & 108 \\
\hline Tennis_Shoes & Vacuum & 32 & 5 & 7 \\
\hline Tennis_Shoes & Vacuum & 32 & 6 & 12 \\
\hline Tennis_Shoes & Vacuum & 32 & 7 & 7 \\
\hline Tennis_Shoes & Vacuum & 32 & 8 & 23 \\
\hline Tennis_Shoes & Vacuum & 32 & 9 & 12 \\
\hline Tennis_Shoes & Vacuum & 32 & 10 & 26 \\
\hline Tennis_Shoes & Vacuum & 32 & 11 & 17 \\
\hline Tennis_Shoes & Vacuum & 32 & 12 & 17 \\
\hline Tennis_Shoes & Vacuum & 33 & 1 & 12 \\
\hline Tennis_Shoes & Vacuum & 33 & 2 & 15 \\
\hline Tennis_Shoes & Vacuum & 33 & 3 & 4 \\
\hline Tennis_Shoes & Vacuum & 33 & 4 & 17 \\
\hline
\end{tabular}




$\begin{array}{llrrr}\text { Tennis_Shoes } & \text { Vacuum } & 33 & 5 & 31 \\ \text { Tennis_Shoes } & \text { Vacuum } & 33 & 6 & 12 \\ \text { Tennis_Shoes } & \text { Vacuum } & 33 & 7 & 12 \\ \text { Tennis_Shoes } & \text { Vacuum } & 33 & 8 & 10 \\ \text { Tennis_Shoes } & \text { Vacuum } & 33 & 9 & 12 \\ \text { Tennis_Shoes } & \text { Vacuum } & 33 & 10 & 23 \\ \text { Tennis_Shoes } & \text { Vacuum } & 33 & 11 & 9 \\ \text { Tennis_Shoes } & \text { Vacuum } & 33 & 12 & 12 \\ \text { Tennis_Shoes } & \text { Vacuum } & 33 & 13 & 25 \\ \text { Tennis_Shoes } & \text { Vacuum } & 33 & 14 & 7 \\ \text { Tennis_Shoes } & \text { Vacuum } & 34 & 1 & 11 \\ \text { Tennis_Shoes } & \text { Vacuum } & 34 & 2 & 16 \\ \text { Tennis_Shoes } & \text { Vacuum } & 34 & 3 & 16 \\ \text { Tennis_Shoes } & \text { Vacuum } & 34 & 4 & 32 \\ \text { Tennis_Shoes } & \text { Vacuum } & 34 & 5 & 13 \\ \text { Tennis_Shoes } & \text { Vacuum } & 34 & 6 & 12 \\ \text { Tennis_Shoes } & \text { Vacuum } & 34 & 7 & 14 \\ \text { Tennis_Shoes } & \text { Vacuum } & 34 & 8 & 8 \\ \text { Tennis_Shoes } & \text { Vacuum } & 35 & 1 & 12 \\ \text { Tennis_Shoes } & \text { Vacuum } & 35 & 2 & 22 \\ \text { Tennis_Shoes } & \text { Vacuum } & 35 & 3 & 8 \\ \text { Tennis_Shoes Vacuum } & 35 & 4 & 21 \\ \text { Tennis_Shoes } & 35 \text { Vacuum } & 35 & 5 & 15 \\ \text { Tennis_Shoes Vacuum } & 35 & 6 & 10 \\ \text { Tennis_Shoes } & \text { Vacuum } & 7 & 20 \\ \text { Tennis_Shoes } & \text { Vacuum } & 9 & 19 \\ \text { Tennis_Shoes } & \text { Vacuum } & & 14\end{array}$

\section{Appendix C: Image Acquisition}

USB drive of all images acquired throughout this research available upon request. Please contact Department of Forensic and Investigative Sciences if desired. 